\title{
A Search for Supersymmetry via Chargino-Neutralino Production in Low- $p$ - Dimuon Channel with the Collider Detector at Fermilab
}

\author{
by \\ Vladimir Rekovic \\ B.S., Guilford College, 1997 \\ M.S., Physics, University of New Mexico, 1999
}

\section{DISSERTATION}

Submitted in Partial Fulfillment of the

Requirements for the Degree of

Doctor of Philosophy

Physics

The University of New Mexico

Albuquerque, New Mexico

May, 2007 
Vladimir Rekovic

Candidate

Physics and Astronomy

Department

This dissertation is approved, and it is acceptable in quality and form for publication on microfilm:

Approved by the Dissertation Committee:
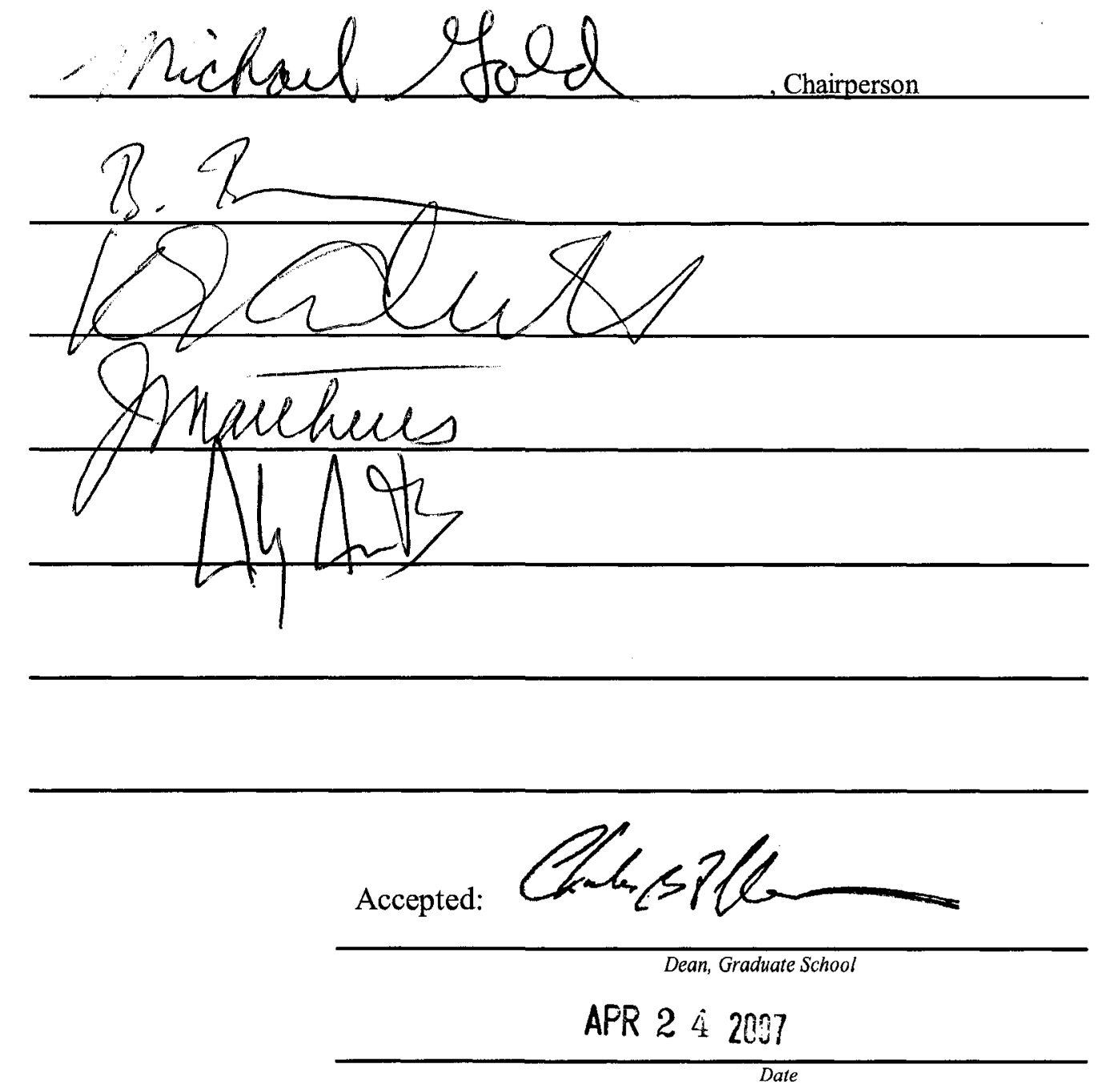


\section{Dedication}

To my parents and my sister. 


\section{Acknowledgments}

I would like to thank my adviser, Michael Gold, and his postdoctoral fellows Steve Worm, Volker Drollinger and John Strologas. Also, Stephan Lammel has provided very useful guidelines and suggestions throughout my work at Fermilab. My work on this analysis has been peer-reffereed by many people in the CDF SUSY group Petter Wittich, Teruki Kamon, Giulia Manca, Beate Heinman, John Zhao, Oscar Gonzalez, Else Lytken, Anadi Canepa, Martin Griffiths, Sunil Dube, Daniela Bartoletto and Thom Junk.

I owe gratitude to many people at the University of New Mexico Physics and Astronomy Department, the staff and all my teachers, especially professors Daniel Finley and Kevin Cahill for introducing me to Quantum Field Theory which awakened my interest for High Energy Physics, professors Igor Gorelov, John Mathews, Bernd Bassalleck, John McGraw and Nebojsa Duric for their support and teachings. I must admit the intellectual debt I owe to my previous teachers at Guilford College: Sheridan Simon, Thom Espinola, Rex Adelberger, and Elwood Parker, and my physics teachers in Uzice, Serbia, Ziko Pavlovic and nastavnik Panic.

This work would have been almost impossible without the boundless love of my parents Mico and Ivanka, and my sister Jelena. I am thankful to my cousins Nikola R., Nikola K., Radmilo, Goran, Djoko, Vera, Strahinja and Maja, my aunts and uncles and my grandparents Vojislav and Dobrina for the trust they had in me. Many thanks to my friends Vasko, Zan, Fernando, Gerardo, Diego, Laura, Reah, Sergio, Cariño, Celia, Alejandro, Sebas, Blanca, Reduan, Adria, Keif, Heidi, Natasa, Eric, May, Tomas, Parick, Yan, Bettina, Gwyneth, Beth, Branko, Borko, Pjer, Vucko, Smilja, Meche, Diana and others for being with me at various moments along this long path. I also thank my primary school teacher Divna Dabic for her wise words and motherly mentorship. 


\title{
A Search for Supersymmetry via Chargino-Neutralino Production in Low- $p_{T}$ Dimuon Channel with the Collider Detector at Fermilab
}

\author{
by \\ Vladimir Rekovic \\ B.S., Guilford College, 1997 \\ M.S., Physics, University of New Mexico, 1999 \\ Ph.D., Physics, University of New Mexico, 2007
}

\begin{abstract}
We have searched for evidence of supersymmetry with $1 \mathrm{fb}^{-1}$ of data collected with low $p_{T}$ di-muon triggers of the Collider Detector on Tevatron Run II, at Fermilab. We looked for trilepton events in $p \bar{p}$ collisions at $\sqrt{s}=1.96 \mathrm{TeV}$. In the Minimal Supersymmetric Standard Model (MSSM) we expect chargino-neutralino pair production, with subsequent decay into three isolated leptons. We observe one event of three isolated muons, a possible hint of supersymmetry.
\end{abstract}

vii 


\section{Contents}

List of Figures $\quad$ xiv

List of Tables $\quad$ xxix

1 Introduction 1

2 Theory 3

2.1 The Standard Model of particle physics . . . . . . . . . . . . 3

2.1.1 Classification of elementary particles . . . . . . . . . 3

2.2 The standard model . . . . . . . . . . . . . . . . 9

2.2.1 Gauge field theories . . . . . . . . . . . . . . 10

2.2.2 Quantum electrodynamics ............... 13

2.2.3 The electroweak theory ................ 14

2.2.4 Spontaneous symmetry breaking: the Higgs mechanism . . . . 15

2.2.5 Quantunchromodynamics ............. 16

2.2 .6 BeyondSM . . . . . . . . . . . . . . . . 17

viii 
Contents

2.3 Supersymmetry . . . . . . . . . . . . . . . . . 21

2.3.1 Trilepton Signal at the Upgraded Tevatron . . . . . . . . . . . 28

3 Experimental Apparatus $\quad 32$

$3.1 \quad$ FermilabAccelerator . . . . . . . . . . . . . . 32

3.2 Collider Detector at Fermilab . . . . . . . . . . . . . . . . 36

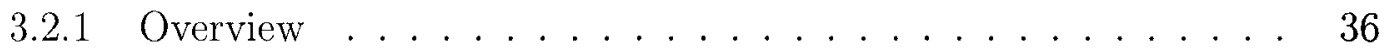

3.2 .2 Tracking System . . . . . . . . . . . . . 40

3.2 .3 Calorimetry ..................... 48

3.2 .4 Muon System . . . . . . . . . . . . . . . . . 5 50

3.2.5 Shower Detector ................... 53

3.2.6 Event Trigger .................... 54

4 Event Reconstruction $\quad 58$

4.1 Lepton Identification . . . . . . . . . . . . . . . . . . . 59

4.1 .1 Electrons ........................ 59

4.1 .2 Muon ...................... 62

4.2 Jet Reconstruction . . . . . . . . . . . . . . . . 65

4.3 Missing Energy . . . . . . . . . . . . . . . . . . . . 69

5 Measurement of Low $p_{T}$ Muon Trigger Efficiencies $\quad 71$

5.1 Trigger Efficiency and Trigger Paths .............. 71 


\section{Contents}

5.1 .1 Total Path Trigger Efficiency . . . . . . . . . . . 72

5.1 .2 ediloh Dataset. . . . . . . . . . . . . . . . . . 74

5.1 .3 L1 and L2 Single-Leg Trigger Efficiency . . . . . . . . . . 76

5.1.4 L3 Single-Leg Trigger Efficiency . . . . . . . . . . . . . . 98

5.1 .5 Summary ...................... 102

6 Muon Isolation Efficiencies $\quad 104$

6.1 Motivation . . . . . . . . . . . . . . . . . 104

6.2 Algorithm and Samples . . . . . . . . . . . . . . . 105

6.2 .1 DY Selection . . . . . . . . . . . . . 105

6.2 .2 ID Variables . . . . . . . . . . . . . . . . . 107

6.2 .3 Backgrounds.................... 109

6.2 .4 Bin-Likelihood .................. 113

6.2.5 Calculation of Isolation Efficiency and Error Propagation . . . . 115

6.2.6 Isolation Efficiency and Scale Factors . . . . . . . . . . . . . 117

6.2 Systematics . . . . . . . . . . . . . 120

7 Measurement of Muon ID Efficiencies in Gen6 Data 123

7.0.8 Muon Identification Cuts . . . . . . . . . . . . . . . . 124

7.1 Sample. . . . . . . . . . . . . . . . . . 125

7.2 Trigger Bias . . . . . . . . . . . . . . . . . 127 
Contents

7.3 Gen $6 \mu$ ID Efficiencies . . . . . . . . . . . . . . . . . . . . . 128

7.4 Summary . . . . . . . . . . . . . . . . . . . 141

8 Trilepton Analysis $\quad 142$

8.1 Search Analysis Strategy . . . . . . . . . . . . . . . . . 142

8.1.1 The observed data . . . . . . . . . . . . . . 143

8.1 .2 The Backgrounds . . . . . . . . . . . . . . . . . 143

8.2 Event Selection . . . . . . . . . . . . . . . . 148

8.3 Weighing of MC . . . . . . . . . . . . . . . . . 149

8.4 Background Estimation . . . . . . . . . . . . . . 151

8.4.1 Heavy flavor background estimation . . . . . . . . . . . . 151

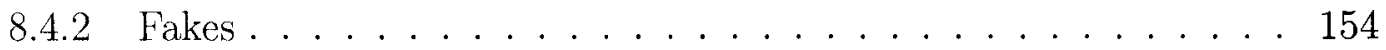

8.4.3 Backgrounds estimated with Monte Carlo . . . . . . . . . . 154

8.5 Systematics . . . . . . . . . . . . . . . . 155

8.6 Control Regions . . . . . . . . . . . . . . . 158

8.6.1 Control Region CON_Z . . . . . . . . . . . . . . 159

8.6.2 Control Region CON_UNM . . . . . . . . . . . . 162

8.6.3 Control Region CON_E . . . . . . . . . . . . . 165

8.6.4 Control Region CON_F . . . . . . . . . . . . 168

8.6.5 Control Region CON_G ................ 171

8.6.6 Control Region CON_H . . . . . . . . . . . . . . . 174 
Contents

8:6.7 Control Region CON_I . . . . . . . . . . . . 177

8.6.8 Control Region CON_J . . . . . . . . . . . . . 180

8.6 .9 Control Region SIG_A . . . . . . . . . . . . 183

8.6.10 Control Region SIG_A2 . . . . . . . . . . . 186

8.7 Opening the box $\ldots \ldots \ldots \ldots$

9 NMH Model $\quad 196$

9.1 Introduction . . . . . . . . . . . . . . 196

9.2 Generation . . . . . . . . . . . . . . . . . . . . . 198

10 References $\quad 202$

$\begin{array}{ll}\text { References } & 203\end{array}$

$\begin{array}{ll}\text { Appendices } & 206\end{array}$

$\begin{array}{ll}\text { A Acceptance with } z \operatorname{Min} A x S L & 207\end{array}$

B Muon Isolation Efficiency as a Function of Openning Angle in gen5 $\begin{array}{ll}\text { Data } & 209\end{array}$

C Resonances Used for gen6 Data ID Efficiencies $\quad 211$

D Cross Check of Muon ID Efficiencies with High $p_{T}$ Group's Results 221

E Efficiencies of Muon ID Cuts for Low and High $p_{T}$ Regions for gen6 224 
Contents

F Comparisson of Efficiencies of Muon ID Cut with High $p_{T}$ Group's 231 


\section{List of Figures}

2.1 Feynman diagrams for fermionic (left) and bosonic (right) contributions to the Higgs's self energy. If the same diagrams exist for both bosons and fermions of exactly the same mass, they cancel exactly . . . . .

2.2 Associated $\tilde{\chi}_{1}^{0} \tilde{\chi}_{2}^{0}$ production in s-channel via virtual $W^{ \pm}$boson exchange (lef) and in $t$-channel via virtual $\tilde{q}$ squark exchange (right) at the Tevatron. For $\tilde{\chi}_{1}^{0}$ and $\tilde{\chi}_{2}^{0}$ masses near $\mathrm{W}$ and $\mathrm{Z}$ boson masses a measurable rate of production is expected at the Tevatron. Since the the above two modes interfere, the $\tilde{\chi}_{1}^{0} \tilde{\chi}_{2}^{0}$ production is enhanced for massive $\tilde{q} \ldots \ldots \ldots \ldots \ldots$

2.3 Thre-body decay modes for charginos (left) and neutralinos (right). These modes occur when the slepton masses are larger than the $\tilde{\chi}_{1}^{0}$ and $\tilde{\chi}_{2}^{0}$ masses so the decays occur via virtual $W^{ \pm}$and $Z^{0}$ bosons (top) or virtual sleptons (botom). . . . . . . . . . . . . . .

2.4 Two-body decay modes for charginos (left) and neutralinos (right). These modes occur when the slepton masses are smaller than the $\tilde{\chi}_{1}^{0}$ and $\tilde{\chi}_{2}^{0}$ masses so the decays occur via real sleptons. . . . . . . . . .

3.1 Schematic drawing of the Fermilab accelerator complex . . . . . . . 


\section{List of Figures}

3.2 Isometric view of the CDF with cut-away quarter . . . . . . . . 37

3.3 Elevation view of one half of the CDF . . . . . . . . . . 38

3.4 Schematic drawing of one quarter of CDF tracking system. Parts of calorimetry system (Plug and End Wall) are also shown on the drawing

3.5 CDF Silicon Vertex Detector - SVXII ..............

3.6 Layer00 viewed in the plane transverse to the beam. Layer00 sensors are colored in red. The two most inner layers of SVXII surrounding Layer00 are shown as well. . . . . . . . . . . . . . . . . . . 44

3.7 Transverse plane view of three COT cells. The $R$-arrow shows the radial direction. Electrostatic field is perpendicular to the field panels and drift velocity is perpendicular to the radius . . . . . . . . . . . . 46

3.8 COT end plates. The wire-plane slots are grouped into eight concentric super layers. The slots are tilted by $35^{\circ}$ with respect to radial direction

3.9 Coverage of muon systems in $\eta-\phi$ space . . . . . . . . . .

3.10 CDF data acquisition and trigger system in Run II . . . . . . . . .

$5.1 J / \psi$ and Z Left: $p_{T}$ of trigger leg (red) and other leg (blue) leg. Right: $p_{T}$ of other leg, numerator (blue), denominator (red) . . . . . . 81

5.2 Zoom-in of $p_{T}$ of denominator (OTHER leg) and numerator. No obvious shoulder at $p_{T}=8 \mathrm{GeV}$, indicating bias by L2 trigger used for our event selection. . . . . . . . . . . . . . . . . 


\section{List of Figures}

$5.3 \mathrm{~J} / \psi$ and $\mathrm{Z}$ After SB subtraction, single-leg L1 efficiency vs $p_{T}$ (top left), vs $\phi$ (top right), vs $\eta$ (bottom). A fit function shown in blue (top right). Number of CMU hits shown in red (top right and bottom left). For $p_{T}>6 \mathrm{GeV} / c$ efficiency becomes flat. Efficiency is approximately flat as a function of $\phi$ and $\eta \ldots \ldots \ldots$. . . . . . . . . .

$5.4 J / \psi$ and Z After SB subtraction, single-leg L2 efficiency vs $p_{T}$ (top left), vs $\phi$ (top right), vs $\eta$ (bottom). A fit function shown in blue (top right). For $p_{T}>6 \mathrm{GeV} / c$ efficiency becomes flat. Efficicncy is approximately flat as a function of $\phi$ and $\eta \ldots . . . . . . .$.

$5.5 \mathrm{~J} / \psi$ and $\mathrm{Z}$ After SB subtraction and $z M i n A x S L$ cut not applied, single-leg L1 efficiency vs $p_{T}$ (top left), vs $\phi$ (top right), vs $\eta$ (bottom). A fit function shown in blue (top right). Number of CMU hits shown in red (top right and bottom left). The absence of $z$ Min AxSL cut lowers the efficiency and introduces a significant $\eta$ dependence. . .

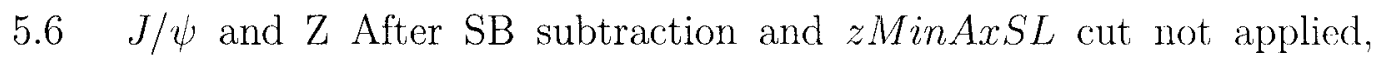
single-leg L2 efficiency vs $p_{T}$ (top left), vs $\phi$ (top right), vs $\eta$ (bottom). A fit function shown in blue (top right). The absence of $z \operatorname{Min} A x S L$ does not affect L2 efficiency which is approximately flat as a function of $\phi$ and $\eta$.

$5.7 \Upsilon$ and Z Left: $p_{T}$ of trigger leg (red) and other leg (bluc) leg. Right: $p_{T}$ of other leg, numerator (blue), denominator (red) . . . . . . .

$5.8 \Upsilon$ and Z After SB subtraction, single-leg L1 efficiency vs $p_{T}$ (top left), vs $\phi$ (top right), vs $\eta$ (bottom). A fit function shown in blue (top right). Number of CMU hits shown in red (top right and bottom left). For $p_{T}>6 \mathrm{GeV} / c$ efficiency becomes flat. Efficiency is approximately

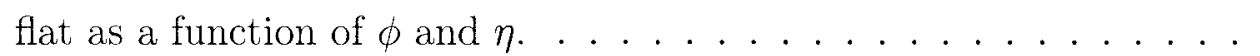


List of Figures

$5.9 \Upsilon$ and Z After SB subtraction, single-leg L2 efficiency vs $p_{T}$ (top left), vs $\phi$ (top right), vs $\eta$ (bottom). A fit function shown in blue (top) right). Number of $\mathrm{CMU}$ hits shown in red (top right and bottom left). For $p_{T}>6 \mathrm{GeV} / c$ efficiency becomes flat. Efficiency is approximatcly

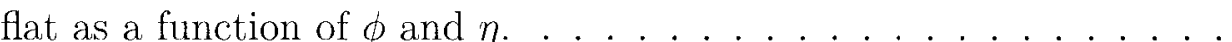

$5.10 \Upsilon$ and $\mathrm{Z}$ after SB subtraction and $z$ Min $A x S L$ cut not applied, singleleg L1 efficiency vs $p_{T}$ (top left), vs $\phi$ (top right), vs $\eta$ (bottom). A fit function shown in blue (top right). Number of CMU hits shown in red (top right and bottom left). The absence of $z M$ in $A x S L$ cut lowers the efficiency and introduces a significant $\eta$ dependence. . . . . . .

$5.11 \Upsilon$ and $\mathrm{Z}$ After SB subtraction and $z M$ in AxSL cut not applied, singlcleg L2 efficiency vs $p_{T}$ (top left), vs $\phi$ (top right), vs $\eta$ (bottom). A fit function shown in blue (top right). The absence of zMinAxSL does not affect L2 efficiency which is approximately flat as a function of $\phi$

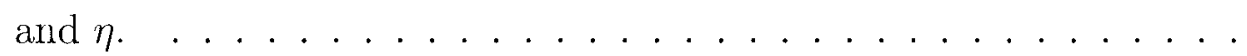

5.12 L1 CMX efficiency in the east arch. The keystone and the miniskirt are excluded. Top, efficiency as a function of $p_{T}$ from $J / \psi$ in black triangles, from $\Upsilon$ red circles, from $Z$ in green squares. Bottom, efficiency obtained from $J / \psi, \Upsilon$ and $\mathrm{Z}$ are combined. Although the $p_{T}$ turn on of the efficiency is not very well resolved, the efficiency is constant for $p_{T}>6$ $\mathrm{GeV} / c \ldots \ldots \ldots \ldots \ldots \ldots \ldots$ 


\section{List of Figures}

5.13 L1 CMX efficiency in the arches as a function of CMX wedge number (left) and CMX stub $\eta$. The keystone and the miniskirt are excluded, which is reflected by the missing bins in the plot on the left. In the same plot, the low efficiency bin corresponds to the keystone part of CMX detector. Wedge \#14 West was malfunctioning during the data taking, so we did not consider the muons found in this wedge. . . .

5.14 L2 CMX efficiency as a function of $p_{T}$, CMX wedge number (right) and CMX stub $\eta$ (bottom). The keystone and the miniskirt are cxcluded.

5.15 L1 CMX efficiency in the west arch. Miniskirts are excluded. Top, efficiency as a function of $p_{T}$ from $J / \psi$ in black triangles, from $\Upsilon$ red circles, from $\mathrm{Z}$ in green squares. Bottom, efficiency obtained from $J / \psi$, $\Upsilon$ and $\mathrm{Z}$ are combined. Although the $p_{T}$ turn on of the efficiency is not very well resolved, the efficiency is constant for $p_{T}>6 \mathrm{GeV} / c . \ldots$.

5.16 L1 CMX efficiency in the arches as a function of CMX wedge number (left) and CMX stub $\eta$. Miniskirts are excluded, which is reflected by the missing bins in the plot on the left. In the same plot, the low efficiency bin corresponds to the keystone part of CMX detector. Wedge \#14 West was malfunctioning during the data taking, so we did not consider the muons found in this wedge. . . . . . . . . . .

5.17 L2 CMX efficiency as a function of $p_{T}$, CMX wedge number (right) and CMX stub $\eta$ (bottom). Miniskirts are excluded. . . . . . . . . .

5.18 L1 CMX efficiency in the miniskirts. Top, efficiency as a function of $p_{T}$ from $J / \psi$ in black triangles, from $\Upsilon$ red circles, from $\mathrm{Z}$ in green squares. Bottom, efficiency obtained from $J / \psi, \Upsilon$ and $\mathrm{Z}$ are combined. 


\section{List of Figures}

5.19 L1 CMX efficiency in the miniskirts as a function of CMX wedge number (left) and CMX stub $\eta$ (right). CMX miniskirt wedges \#15 and \#20 were not in function during this data taking so the bins corresponding to these wedges are missing from the plot on the left. . . . 96

5.20 L2 CMX efficiency in the miniskirts as a function of $p_{T}$, CMX wedge number (right) and CMX stub $\eta$ (bottom) . . . . . . . . .

5.21 L3 CMU. Left: $p_{T}$ distribution of the denominator (red) and the numerator (blue). Right: L3 CMU trigger efficiency. In the first bin, the inefficiency is due to $p_{T}$ cut of $4 \mathrm{GeV}$ at L3, almost entirely. . . . . . 100

5.22 L3 CMX. Left: $p_{T}$ distribution of the denominator (red) and the numerator (blue). Right: L3 CMX trigger efficiency. There is much lower statistics in for this measurement to L3 CMU measurement, because CMX of much lower yield of CMX muons.

6.1 Selection of events shown for a part of edilod DATA: (a) number of CdfMuons in the event, require 2 at least, (b) Difference between the $z_{0}$ coordinates of the muons' COT tracks in $\mathrm{cm}$, (c) COT exit radius in $\mathrm{cm}$, require $\rho_{\text {cxit }}>$ $140 \mathrm{~cm}$ (d) $z$ coordinate of selected primary vertcx in $\mathrm{cm}$, require $\left|z 0_{\text {prim }}\right|<$

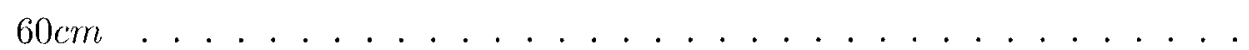

6.2 Selection of events $\mu \mu$ pairs for all DATA (blue) and MC (red). Top: invariant mass $\left(\mathrm{m}_{\mu \mu}\right)$ of muon pair. For reference we show $\mathrm{m}_{\mu \mu}$ of DATA when both legs isolated (green). Selected events have $\mathrm{m}_{\mu \mu \nu}>12 \mathrm{GeV}$ to assure absence of $J / \Psi$ and $\Upsilon$ resonances. Bottom: difference of two legs' COT tracks angles, i.e. opening angle $\Delta \phi$. Selected events shown in filled blue $\Delta \phi>150^{\circ} \ldots \ldots \ldots$. . . . . . . . . . . 108

xix 


\section{List of Figures}

$6.3 \mu$ ID variables. One leg (black), testing leg (blue), MC testing log (red). All ID cuts, except for D0 cut, applied to the first leg. Only the matching cuts ( $\operatorname{cmup} \Delta \mathrm{x}$ and $\chi_{c m u p}^{2}$ ) and track quality cut (number of COT hits) applied on the second leg, i.e. testing leg. . . . . . . . . . 110

$6.42^{\text {nd }} \operatorname{leg} p_{T}$ : for unlike sign (US) pairs on the left and like sign (LS) pairs on the right. For reference, MC DY normalized to the $\mathrm{Z}$ peak overlaid in red on the left. Negligible number of LS MC events not shown on the right. (a) US pairs (b) LS pairs, (c) US pairs 1 leg isolated, (d) US pairs 1 leg isolated, (e) US pairs both legs isolated, (f) LS pairs both legs isolated. On the right, for LS pairs, a fit with a Gaussian function is shown in black line. . . . . . . . . . . . . . . . . . . 111

$6.5 m_{\mu \mu}: \operatorname{leg} p_{T}$ : for unlike sign (US) pairs on the left and like sign (LS) pairs on the right. For reference, MC DY normalized to the $\mathrm{Z}$ peak, overlaid in red on the left. Negligible number of LS MC events not, shown on the right. (a) US pairs (b) LS pairs, (c) US pairs $1 \mathrm{leg}$ isolated, (d) US pairs 1 leg isolated, (e) US pairs both legs isolated, (f) LS pairs both legs isolated. On the right, for LS pairs, a fit with a exponential function is shown in black line. . . . . . . . . . . 112

6.6 Bin-Likelihood fit (a) fit to pr of the $2^{\text {nd }}$ leg for events where one log isolated (b) fit to $\mathrm{p} T$ of the $2^{\text {nd }}$ leg for events where both legs isolated . . . . . . 113

6.7 Bin-Likelihood fit (a) fit to $\mathrm{m}_{\mu \mu}$ for events where one leg isolated (b) fit to $\mathrm{m}_{\mu \mu}$ for events where both legs isolated . . . . . . . . . . . 113 


\section{List of Figures}

6.8 Fixed isolation cut of $2 \mathrm{GeV}$ for $\mathrm{p}_{T}<20 \mathrm{GeV}$. LEFT: muon Pt. Unlike Sign (US) in light blue, US one leg iso in medium dark blue, US both leg isolated dark blue. Like Sign (LS) in green. LS one leg iso in medium dark green, LS both leg isolated dark green. CENTER: Isolation efficiency vs $\mathrm{p}_{T}$. RIGHT: Isolation efficiency corrected for LS background. . . . . . . . . . . . . . . . . 118

6.9 Fixed isolation cut of $2 \mathrm{GeV}$ for $\mathrm{p}_{T}<20 \mathrm{GeV}$. LEFT: muon Mass. Unlike Sign (US) in light blue, US one leg iso in medium dark blue, US both leg isolated dark blue. Like Sign. (LS) in green. LS one leg iso in medium dark green, LS both leg isolated dark green. CENTER: Isolation efficiency vs $\mathrm{p}_{T}$. RIGHT: Isolation efficiency corrected for LS background. . . . . . . . . . . . . . . . . 119

6.10 No smoothing of HF LS distributions but used the distribution "as is". Results for $\mathrm{p}_{T}$ (left set of three histograms) and results for $\mathrm{m}_{\mu \mu}$ (right three histograms). LEFT: muon Pt. Unlike Sign (US) in light blue, US one leg iso in medium dark blue, US both leg isolated dark blue. Like Sign (LS) in green. LS one leg iso in medium dark green, LS both leg isolated dark green. CENTER: Isolation efficiency vs $\mathrm{p}_{T}$. RIGHT: Isolation efficiency corrected for LS background. . . . . . . . . . . . . . . . . . . . . . 121

6.11 Applied all ID cuts, including calorimetry cuts, on both legs. Results for $\mathrm{p}_{T}$ (left set of three histograms) and results for $\mathrm{m}_{\mu \mu}$ (right three histograms). LEFT: muon Pt. Unlike Sign (US) in light blue, US one leg iso in medium dark blue, US both leg isolated dark blue. Like Sign (LS) in green. LS one leg iso in medium dark green, LS both leg isolated dark green. CENTER: Isolation efficiency vs $\mathrm{p}_{T}$. RIGHT: Isolation efficiency corrected for

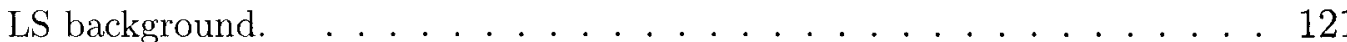




\section{List of Figures}

6.12 Fixed isolation cut of $1 \mathrm{GeV}$ for $\mathrm{p}_{T}<10 \mathrm{GeV}$.Results for $\mathrm{p}_{T}$ (left set of three histograms) and results for $\mathrm{m}_{\mu \mu}$ (right three histograms). LEFT: muon Pt. Unlike Sign (US) in light blue, US one leg iso in medium dark blue, US both leg isolated dark blue. Like Sign (LS) in green. LS one leg iso in medium dark green, LS both leg isolated dark green. CENTER: Isolation efficiency vs $\mathrm{P}_{T}$. RIGHT: Isolation efficiency corrected for LS background. . . . . . 122

7.1 JU mass for all isolated dimuons (blue) and dimuons in the same CMU wedge (black, filled). This is only relevant to $\mathrm{CMU}(\mathrm{P})$ fiducial muons. Triggering CMUP muon cannot physically be in the same wedge as a CMX fiducial muon.

$7.2 J / \Psi$ (top) and Z (bottom) before (left) and after (right) isolation applied to both legs. On the right, signal (blue) and background (red) regions. ...................... 127

7.3 $p_{T}$ of the trigger leg (red) and the other leg (blue). . . . . . . . 130

7.4 CMU, CMUP, and CMX ID efficiencies as a function of $p_{T}$ of other leg (blue circles) and trigger leg (reg triangles), in the low $p_{T}$ region $\left(p_{T}\right.$

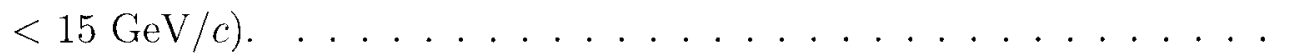

7.5 CMU, CMUP, and CMX ID efficiencies as a function of $p_{T}$ of other leg (blue circles) and trigger leg (reg triangles), in the high $p_{T}$ region $\left(p_{T}\right.$ $>15 \mathrm{GeV} / c)$.

7.6 In LOW $p_{T}$ region, CMU ID variables with cuts applied on all but the plotted variables. .................... 133

7.7 In LOW $p_{T}$ region, CMUP ID variables with cuts applied on all but the plotted variables. . . . . . . . . . . . . . . . . . 134 


\section{List of Figures}

7.8 In LOW $p_{T}$ region, CMUP ID variables with cuts applied on all but the plotted variables. . . . . . . . . . . . . . . . . 135

7.9 In LOW $p_{T}$ region, CMX ID variables with cuts applied on all but the plotted variables. . . . . . . . . . . . . . 136

7.10 In high $p_{T}$ region, CMU ID variables with cuts applied on all but the plotted variables.

7.11 In high $p_{T}$ region, CMUP ID variables with cuts applied on all but the plotted variables.

7.12 In high $p_{T}$ region, CMUP ID variables with cuts applied on all but the plotted variables.

7.13 In high $p_{T}$ region, CMX ID variables with cuts applied on all but the plotted variables.

8.1 Dimuon mass for data, SM prediction, and SUSY MC signal in region

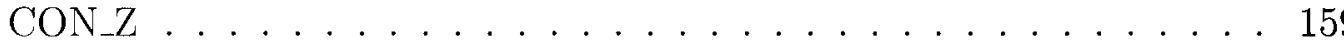

8.2 $\quad E_{\mathrm{T}}$ for data, SM prediction, and SUSY MC signal in region CON_Z $\quad 160$

8.3 Dimuon mass for data, SM prediction, and SUSY MC signal in region

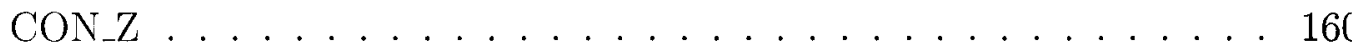

8.4 $E_{\mathrm{T}}$ for data, SM prediction, and SUSY MC signal in region CON Z . 161

8.5 Dimuon mass for data, SM prediction, and SUSY MC signal in region

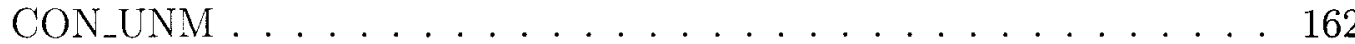

8.6 $\quad E_{\mathrm{T}}$ for data, SM prediction, and SUSY MC signal in region CON_UNM 163

8.7 Dimuon mass for data, SM prediction, and SUSY MC signal in region

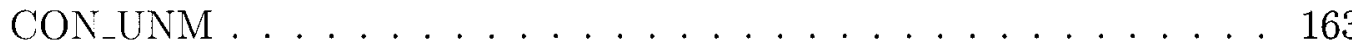




\section{List of Figures}

8.8 $E_{\mathrm{T}}$ for data, SM prediction, and SUSY MC signal in region CON_UNM 164

8.9 Dimuon mass for data, SM prediction, and SUSY MC signal in region

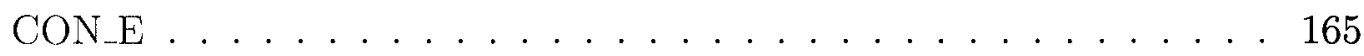

8.10 $\mathrm{E}_{\mathrm{T}}$ for data, SM prediction, and SUSY MC signal in region CON_E . 166

8.11 Dimuon mass for data, SM prediction, and SUSY MC signal in region

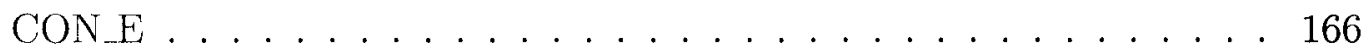

8.12 $E_{\mathrm{T}}$ for data, SM prediction, and SUSY MC signal in region CON_E . 167

8.13 Dimuon mass for data, SM prediction, and SUSY MC signal in region

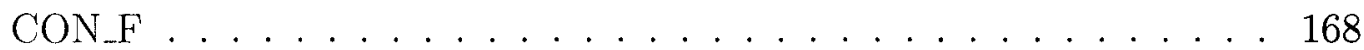

8.14 $E_{\mathrm{T}}$ for data, SM prediction, and SUSY MC signal in region CON_F $\quad$. 169

8.15 Dimuon mass for data, SM prediction, and SUSY MC signal in region

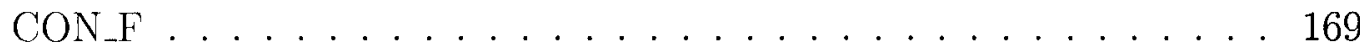

8.16 $E_{\mathrm{T}}$ for data, SM prediction, and SUSY MC signal in region CON_F . 170

8.17 Dimuon mass for data, SM prediction, and SUSY MC signal in region

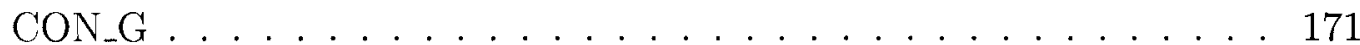

8.18 $E_{\mathrm{T}}$ for data, SM prediction, and SUSY MC signal in region CON_G . 172

8.19 Dimuon mass for data, SM prediction, and SUSY MC signal in region

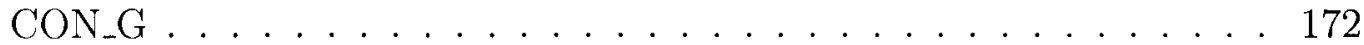

8.20 $E_{\mathrm{T}}$ for data, SM prediction, and SUSY MC signal in region CON_G $\quad 173$

8.21 Dimuon mass for data, SM prediction, and SUSY MC signal in region

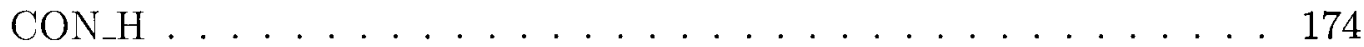

$8.22 E_{\mathrm{T}}$ for data, SM prediction, and SUSY MC signal in region CON_H . 175 xxiv 


\section{List of Figures}

8.23 Dimuon mass for data, SM prediction, and SUSY MC signal in region

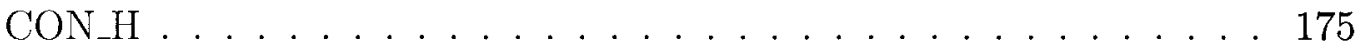

8.24 $E_{\mathrm{T}}$ for data, SM prediction, and SUSY MC signal in region CON_H . 176

8.25 Dimuon mass for data, SM prediction, and SUSY MC signal in region

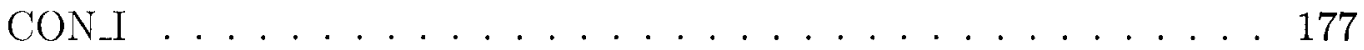

8.26 $E_{\mathrm{T}}$ for data, SM prediction, and SUSY MC signal in region CON_I . . 178

8.27 Dimuon mass for data, SM prediction, and SUSY MC signal in region

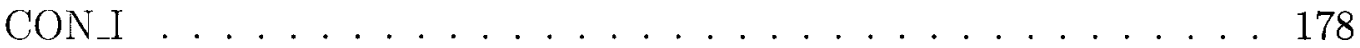

8.28 $\mathrm{K}_{\mathrm{T}}$ for data, SM prediction, and SUSY MC signal in region CON_I . . 179

8.29 Dimuon mass for data, SM prediction, and SUSY MC signal in region CON_J ................................ 180

8.30 $\mathrm{E}_{\mathrm{T}}$ for data, SM prediction, and SUSY MC signal in region CON_J . . 181

8.31 Dimuon mass for data, SM prediction, and SUSY MC signal in region

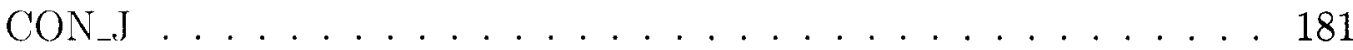

$8.32 E_{\mathrm{T}}$ for data, SM prediction, and SUSY MC signal in region CON_J . . 182

8.33 Dimuon mass for data, SM prediction, and SUSY MC signal in region

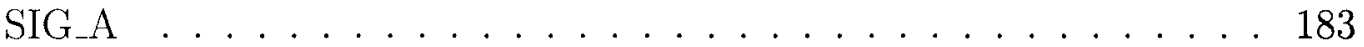

8.34 $E_{\mathrm{T}}$ for data, SM prediction, and SUSY MC signal in region SIG_A . . 184

8.35 Dimuon mass for data, SM prediction, and SUSY MC signal in region

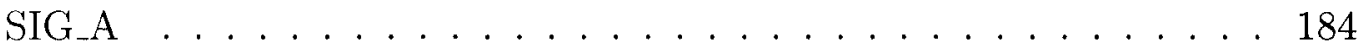

8.36 $E_{\mathrm{T}}$ for data, SM prediction, and SUSY MC signal in region SIG_A . . 185 


\section{List of Figures}

8.37 Dimuon mass for data, SM prediction, and SUSY MC signal in region $\mathrm{SIG}_{-} \mathrm{A} 2 \ldots \ldots \ldots \ldots \ldots$

8.38 $E_{\mathrm{T}}$ for data, SM prediction, and SUSY MC signal in region SIG_A2 . 187

8.39 Dimuon mass for data, SM prediction, and SUSY MC signal in region

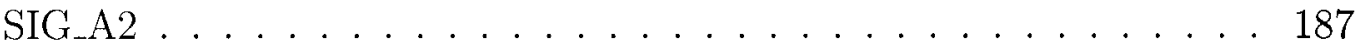

8.40 $\mathbb{E}_{\mathrm{T}}$ for data, SM prediction, and SUSY MC signal in region SIG_A2 . 188

8.41 Transverse plane display of the observed trimuon event in the CDF detector. Muons are shown in green lines. Only the tracks with $p_{T}>$ $1 \mathrm{GeV} / c^{2}$ are shown (blue lines) and the calorimetry towers with $E_{T}>$ $0.7 \mathrm{GeV} \ldots \ldots \ldots \ldots \ldots \ldots$

$8.42 \eta-\phi$ Lego display of $E_{T}$ in the observed trimuon event in the CDF detector. Muons are shown in green lines. Photons are shown in yellow. Only the calorimetry towers with $E_{T}>0.7 \mathrm{GeV} \ldots \ldots . \ldots 191$

8.43 Dimuon mass for data, SM prediction, and SUSY MC signal in region $\mathrm{SIG}_{-} \mathrm{A} \ldots \ldots \ldots \ldots \ldots$

8.44 Dimuon mass (1+3) for data, SM prediction, and SUSY MC signal in region SIG_A

8.45 Dimuon mass $(2+3)$ for data, SM prediction, and SUSY MC signal in region $\mathrm{SIG}_{-} \mathrm{A} \ldots \ldots \ldots \ldots \ldots$

8.46 $1^{\text {st }}$ muon $p_{T}$ for data, SM prediction, and SUSY MC signal in region

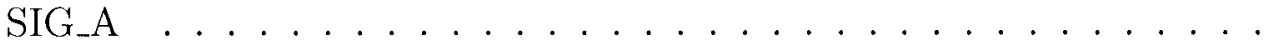

$8.472^{\text {nd }}$ muon $p_{T}$ for data, SM prediction, and SUSY MC signal in region 


\section{List of Figures}

$8.483^{\text {rd }}$ muon $p_{T}$ for data, SM prediction, and SUSY MC signal in region $\mathrm{SIG}_{-} \mathrm{A} \ldots \ldots \ldots \ldots \ldots \ldots$

8.49 Jet multiplicity for data, SM prediction, and SUSY MC signal in region

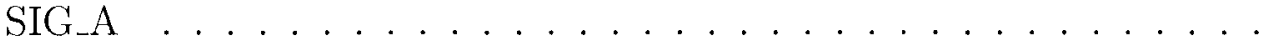

8.50 3-D $\Delta \phi$ of the first two muons for data, SM prediction, and SUSY MC signal in region SIG_A . . . . . . . . . . . . . . . .

A.1 Top: zMinAxSL, in green events with zMinAxSL $<1.5 \mathrm{~cm}(1 / 6$ of total), for TRIGGER and OTHER leg. Bottom: z0 for TRIGGER and OTHER leg . . . . . . . . . . . . . . . 208

B.1 Isolation efficiency vs $\Delta \phi\left(1, \mathrm{j}_{2}\right)$ segment. Fixed energy cut of 2 for $\mathrm{p}_{T}<$ $20 \mathrm{GeV} / \mathrm{c}$, otherwise ConeR4Et $/ p_{T}<0.1$ cut.

C.1 $J / \psi$ mass distributions in pt bins, used in efficiency calculations. Numerators are in blue, filled, histograms, and denominators in red, not filled, histograms.

C.2 $\Upsilon$ mass distributions in pt bins, used in efficiency calculations. Numerators are in blue, filled, histograms, and denominators in red, not filled, histograms. . . . . . . . . . . . . . . . . .

C.3 Z mass distributions in pt bins, used in efficiency calculations. Numerators are in blue, filled, histograms, and denominators in red, not filled, histograms. . . . . . . . . . . . . . . . . .

C.4 $J / \psi$ mass distributions in phi bins, used in efficiency calculations. Numerators are in blue, filled, histograms, and denominators in red, not filled, histograms. 


\section{List of Figures}

C.5 $\Upsilon$ mass distributions in phi bins, used in efficiency calculations. Numerators are in blue, filled, histograms, and denominators in red, not filled, histograms. . . . . . . . . . . . . . . . . 216

C.6 Z mass distributions in phi bins, used in efficiency calculations. Numerators are in blue, filled, histograms, and denominators in red, not filled, histograms. . . . . . . . . . . . . . . 217

C.7 $J / \psi$ mass distributions in eta bins, used in efficiency calculations. Numerators are in blue, filled, histograms, and denominators in red, not filled, histograms. . . . . . . . . . . . . . . . . . . . 218

C.8 $\Upsilon$ mass distributions in eta bins, used in efficiency calculations. Numerators are in blue, filled, histograms, and denominators in red, not filled, histograms. . . . . . . . . . . . . . . . . . . . 219

C.9 Z mass distributions in eta bins, used in efficiency calculations. Numerators are in blue, filled, histograms, and denominators in red, not filled, histograms. . . . . . . . . . . . . . . . .

D.1 CMU, CMUP, and CMX ID efficiencies of Top Group's all cuts as a function of $p_{T}$ of other leg (blue circles) and trigger leg (reg triangles), in the high $p_{T}$ region $\left(p_{T}>20 \mathrm{GeV} / c\right)$ 


\section{List of Tables}

2.1 List of known (discovered) Standard Model elementary particles and some of their properties. There are 12 fermionic particles (first two raws) and five bosonic particles (last raw). Postulated but not yet discovered bosons are graviton and Higgs boson. . . . . . . . . . 5

2.2 Fermions can be divided in three generations, each consisting of a lep-

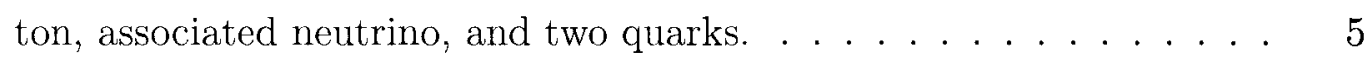

2.3 List of mesons, their quark content and mass in $\mathrm{MeV} \ldots \ldots \ldots$

2.4 List of baryons, their quark content and mass in $\mathrm{MeV} \ldots \ldots \ldots .7$

2.5 Not yet discovered particles in MSSM. Gauge eigen states if denonted as "- ii $-"$ are the same as mass eigen states . . . . . . . . . . . . .

3.1 COT layers summary $\ldots \ldots \ldots \ldots \ldots \ldots \ldots \ldots$

4.1 Electron ID cuts. Electron object must pass these cuts to be considered in this analysis . . . . . . . . . . . . .

4.2 Muon ID cuts. Muon object must pass these cuts to be considered in this analysis $\ldots \ldots \ldots \ldots \ldots \ldots \ldots \ldots$

xxix 


\section{List of Tables}

5.1 Three groups of runs for which different SUSY_DILEPTON triggers were used. Second column gives lowest and highest run number, third column is version of SUSY_DILEPTON trigger dataset used, fourth column is version of PHYSICS_3 table used, fifth column is integrated luminosity collected. Note that run ranges are not contiguous. . . .

5.2 Trigger paths in SUSY_DILEPTON_2_v-5. Note the presence of single electron path. Also note the presence of DIMUON paths with single muon triggers at L2 (\#16 and \# 18) . . . . . . . . . . . 76

5.3 Trigger paths in SUSY_DILEPTON_3_v-1. Note the presence of CMUP paths and the absence of CMU paths. . . . . . . . . . .

5.4 Trigger paths in SUSY_DILEPTON_3_v-2. Note the presence of CMUP paths and the absence of CMU paths. . . . . . . . . . . . 77

5.5 CMU trigger efficiency fit function parameters by trigger level. L1' and L1 entries list the efficiency fit function parameters for Level 1 with and without $z \operatorname{Min} A x S L$ cut imposed, respectively. The effect of this cut is negligible for L2. Parameters extracted from figures 5.3, 5.5, 5.4, 5.21.102

5.6 CMX trigger efficiency fit function parameters by trigger level for tho East and West CMX Arch. Result for L3 is obtained from muons from both East and West CMX Arches. Parameters extracted from figures $5.12,5.15,5.14,5.17$ and $5.22 \ldots \ldots \ldots \ldots 2$

5.7 Miniskirt CMX trigger efficiency fit function parameters by trigger level. Parameters extracted from figures $5.18,5.20$ and 5.22. . . . . 103

6.1 ID cuts for muons: CMU-muon has a CMU stub, CMUP-muon has both CMU and CMP stubs, and a CMX-muon a CMX stub . . . . . . 109 


\section{List of Tables}

6.2 Bin-Likelihood factors for $\mathrm{p}_{T}$ HF LS and MC DY distributions, also shown in middle row $\left(1_{\text {iso }}\right.$ case $)$ and bottom row $\left(2_{i \text { iso }}\right.$ case $)$ of Figure 6.5 where HF LS is on right (green histogram) and MC DY is on rihgt (red markers). The sum of apropriately weighted HF LS and MC DY histogram approximate DATA US histgram on right (blue histogram)

6.3 Bin-Likelihood factors for $\mathrm{m}_{\mu \mu}$ HF LS and MC DY distributions, also shown in middle row $\left(1_{\text {iso }}\right.$ case $)$ and bottom row $\left(2_{i s o}\right.$ case) of Figure 6.4 where HF LS is on right (green histogram) and MC DY is on rihgt (red markers). The sum of apropriately weighted HF LS and MC DY histogram approximate DATA US histgram on right (blue histogram)

6.4 Isolations efficiencies and scale factors for $3 \mathrm{p}_{T}$ bins: ratio of efficiency measured in the data to the Monte Carlo. The errors in the data are statistical. For scale factors we included sytematical erros discussed in the subsection "Systematics" . . . . . . . . . . . . . . . . 117

6.5 Isolation efficiencies and scale factors for $3 \mathrm{~m}_{\mu \mu}$ bins: ratio of efficiency measured in the data to the Monte Carlo. The errors in the data are statistical. For scale factors we included sytematical crros discussed in the subsection "Systematics". . . . . . . . . . . . . . .

7.1 CMU ID efficiencies (gen6 and gen5 reported in CDF7210) for low $p_{T}$ $(<15 \mathrm{GeV} / \mathrm{c})$ and $h i g h p_{T}(>20 \mathrm{GeV} / \mathrm{c})$ regions. Efficiencies are listed for each cut applied individually. The efficiency of all the cuts applied together is listed in the last entry, "ALL". This was not the case for gen5, documented in CDF Note $7210 . \ldots \ldots . \ldots . \ldots 128$

$\mathrm{xxxi}$ 
List of Tables

7.2 CMUP ID efficiencies (gen6 and gen5 reported in CDF7210) for low $p_{T^{\prime}}(<15 \mathrm{GeV} / \mathrm{c})$ and high $p_{T}(>20 \mathrm{GeV} / \mathrm{c})$ regions. Efficiencies are listed for each cut applied individually. The efficiency of all the cuts applied together is listed in the last entry, "ALL". This was not the case for gen5, documented in CDFNote $7210 \ldots \ldots \ldots$. . . . . .

7.3 CMX ID efficiencies (gen6 and gen5 reported in CDF7210) for low $p_{T}$ $(<15 \mathrm{GeV} / \mathrm{c})$ and high $p_{T}(>20 \mathrm{GeV} / \mathrm{c})$ regions. Efficiencies are listed for each cut applied individually. The efficiency of all the cuts applied together is listed in the last entry, "ALL". Note, that for gen6 results, we separately measured efficiencies from $J / \psi$ and $\Upsilon$. This was not the case for gen5, documented in CDFNote 7210. . . . . . . . . . .

8.1 The muon ID scale factors (systematic uncertainty shown second) . . 150

8.2 The muon Isolation scale factors (systematic uncertainty shown second) 150

8.3 The electron ID x Isolation scale factors for gen5 (systemic uncertainty shown second) . . . . . . . . . . . . . . . 150

8.4 The electron ID x Isolation scale factors for gen $6 \ldots \ldots$. . . . . . 150

8.5 Fit of di-muon invariant mass for opposite sign pairs. . . . . . . . . . 152

8.6 Fit of di-muon invariant mass for same sign pairs. . . . . . . . . 153

8.7 Systematic errors for the backgrounds in SIG A region. . . . . . . . 156

8.8 Systematic errors for the SIG1 point in SIG A region. . . . . . . . . 157

8.9 Systematic errors for the SIG2 point in SIG A rcgion. . . . . . . . . 157

xxxii 
List of Tables

8.10 Expected and observed number of dilepton and trilepton events in our control regions. We observe one trilepton event in the signal region $\mathrm{SIG}_{-} \mathrm{A} \ldots \ldots \ldots \ldots \ldots \ldots$

8.11 Properties of the observed event in region $\mathrm{SIG}_{-} \mathrm{A} \ldots \ldots$. . . . . . . 190

9.1 For each $\mathrm{m}_{0}$ point, number of events generated and produced using cdfsoft MCProduction, and a location of corresponding subdirectory, sequential output files and $\log$ files in this subdirectory of directory fcdfdata121:/export/data3/exotic/rekovic/rexo0b/. For points $m_{0}=90$, 120, 150 CAF jobs crashed, so the output sequential files are not avail-

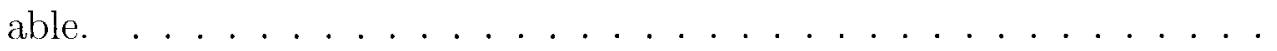

9.2 For each $\mathrm{m}_{0}$ point, location of root files generated using cdfsoft Stutuple_v_243, in the directory fcdflnx6:/ export/ data3/ cdfmc/ rekovic/ $\mathrm{MC} / 6.1 .2 / \mathrm{rexo0b} / \ldots \ldots \ldots \ldots \ldots$

9.3 For each $\mathrm{m}_{0}$ point of the NMH model with above described parameters, theoretical cross section given by PYTHIA, luminosity of MC sample (i.e. number of events generated $/ \sigma_{r H}$ ), number of tri-muon events that pass cuts of our analysis in low $p_{T}$ di-muon channel, and number of expected events to be observed with $1 \mathrm{fb}^{-1}$ data collected with CDF

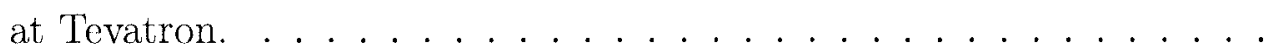

D.1 CMUP ID efficiencies of cuts used by the Top (high $p_{T}$ ) group and efficiencies of these cuts measured by the Top group and documented in CDF7956 (both for gen6 data). . . . . . . . . . . . . . 222

D.2 CMX ID efficiencies of cuts used by the Top (high $p_{r}$ ) group and efficiencies of these cuts measured by the Top group and documented in CDF7956 (both for gen6 data). . . . . . . . . . . . . . 222

xxxiii 


\section{List of Tables}

E.1 individual cut eff CMU low pt (EM,HAD,CAL,D0,TRK,CMUDX) for trigger (red) and other (blue) leg. . . . . . . . . . . . . . 225

E.2 individual cut eff CMU high pt (EM,HAD,CAL,D0,TRK,CMUDX) for trigger (red) and other (blue) leg. . . . . . . . . . . . . 226

E.3 individual cut eff CMUP low pt (EM, HAD, CAL, D0, TRK, CMUDX, CMPDX) for trigger (red) and other (blue) leg. . . . . . . . . . . . 227

E.4 individual cut eff CMUP high pt (EM, HAD, CAL, D0, TRK, CMUDX, CMPDX) for trigger (red) and other (blue) leg. . . . . . . . . . . . 228

E.5 individual cut eff CMX low pt (EM,HAD,CAL,D0,TRK,CMXDX) for trigger (red) and other (blue) leg. . . . . . . . . . . . . . . 229

E.6 individual cut eff CMX high pt (EM,HAD,CAL,D0,TRK,CMXDX) for trigger (red) and other (blue) leg. . . . . . . . . . . . . . 230

F.1 Top Group's individual cut eff CMUP high pt (EM, HAD, ISO, D0, TRK, CMUDX, CMPDX) for trigger (red) and other (blue) leg. . . . 232

F.2 TopGroup's individual cut eff CMX high pt (EM, HAD, ISO, D0, TRK, CMXDX) for trigger (red) and other (blue) leg. . . . . . . . . . . . 233 


\section{Chapter 1}

\section{Introduction}

Particle, or high energy, physics is the study of quarks and leptons and their interactions. The theory describing quarks and leptons is known as Standard Model. It is a combination of two other theories, the electroweak (EWK) theory of Weinberg, Salam and Glashow, and Quantum Chromodynamics (QCD). Quantum Electrodynamics (QED) is a part of EWK theory and it describes interactions of electrically charged particles and photons. Its predictive power makes it one of the most successful theories in all science. The reminder of EWK theory describes charged and neutral weak interactions. QCD describes the interaction of quarks via the strong force.

Overall, the Standard Model has been very successful in predicting experimental results. However, it is considered to be only a low energy effective theory of some more fundamental theory. Class of theories with Supersymmetry (SUSY) are viable candidates for such a theory. This dissertation is a search for evidence beyond the Standard Model by searching for SUSY signature in the data collected with CDF detector at Tevatron Run II. Chapter 2 describes the theory examined, Chapter 3 the apparatus used, Chapter 4 the data processing, Chapter 5,6 and 7 the measurements of efficiencies we needed and performed for the analysis, Chapter 8 the analysis. We 
Chapter 1. Introduction

conclude with Chapter 9 where we discuss one SUSY model and its acceptance in di-muon low $p_{T}$ channel we used in this analysis. 


\section{Chapter 2}

\section{Theory}

Supersymmetry has been one of the theoretical models giving a description of physics beyond the SM. This chapter provides a general description of the SM in Section II.1. The theoretical models beyond SM are presented in Section II.2, with a more detailed treatment of Supersymmetry and the phenomenological framework of the Minimal Supersymmetric Standard Model (MSSM) in Section II.3. Minimal Supersymmetric Gravity (mSUGRA) and mSUGRA-like models are discussed in section II.4.

\subsection{The Standard Model of particle physics}

\subsubsection{Classification of elementary particles}

In the past there have been many models that attempted to describe the interactions of elementary particles. The most successful one has become known as the Standard Model. Its success is depicted in robustness and precision to which it is able to predict results of these interactions. SM has withstood the test of physics phenomena on the scale as low as $10^{-} 19 \mathrm{~m}$. 


\section{Chapter 2. Theory}

A particle is considered to be elementary if it does not exhibit any substructure. SM formulation is based on the a quantum gauge field theory that determines the interactions between the elementary particles. It organizes elementary particles based on the type of the interactions. Presently, there are total of twelve known elementary particles that constitute matter and eight particles responsible for interactions between them. Matter particles are fermions and interaction particles are bosons, and they are fundamentally different. Elementary particles can be categorized as follows:

1. Hadrons: these exist of quarks and can be categorized in:

(a) Baryons: these exist of 3 quarks or 3 antiquarks.

(b) Mesons: these exist of one quark and one antiquark.

2. Leptons: $\mathrm{e}^{ \pm}, \mu^{ \pm}, \tau^{ \pm}, \nu_{\mathrm{e}}, \nu_{\mu}, \nu_{\tau}, \bar{\nu}_{\mathrm{e}}, \bar{\nu}_{\mu}, \bar{\nu}_{\tau}$.

3. Field quanta: $\gamma, \mathrm{W}^{ \pm}, \mathrm{Z}^{0}$, gluons, gravitons and a Higgs boson. ${ }^{1}$

An overview of particles and antiparticles is given in the following table:

Here $B$ is the baryon number and $L$ the lepton number. It is found that there are three different lepton numbers, one for e, $\mu$ and $\tau$, which are separately conserved. $\mathrm{T}$ is the isospin, with $T_{3}$ the projection of the isospin on the third axis, $\mathrm{C}$ the charmness, $\mathrm{S}$ the strangeness and $\mathrm{B}^{*}$ the bottomness. The anti particles have quantum numbers with the opposite sign except for the total isospin $\mathrm{T}$.

The fermionic particles can be classified into three families, shown in Table 2.2. Only the 1 st generation particles are stable and therefore form ordinary matter. The heavier, higher generation particles decay into the first generation particles.

Unlike leptons, quarks cannot be observed in a free state. Quarks exist in three colors but because they are confined these colors cannot be seen directly. The color

\footnotetext{
${ }^{1}$ Higgs boson and gravitons are not discovered, yet.
} 
Chapter 2. Theory

\begin{tabular}{|c|c|c|c|c|c|c|c|c|c|c|}
\hline Particle & $\operatorname{spin}(\hbar)$ & $\mathrm{B}$ & $\mathrm{L}$ & $\mathrm{T}$ & $\mathrm{T}_{3}$ & $\mathrm{~S}$ & $\mathrm{C}$ & $\mathrm{B}^{*}$ & charge $(e)$ & $m_{0}(\mathrm{MeV})$ \\
\hline $\bar{d}$ & $1 / 2$ & $1 / 3$ & 0 & $1 / 2$ & $-1 / 2$ & 0 & 0 & $\overline{0}$ & $-1 / 3$ & 9 \\
\hline $\mathrm{u}$ & $1 / 2$ & $1 / 3$ & 0 & $1 / 2$ & $1 / 2$ & 0 & 0 & 0 & $+2 / 3$ & 5 \\
\hline $\mathrm{s}$ & $1 / 2$ & $1 / 3$ & 0 & 0 & 0 & -1 & 0 & 0 & $-1 / 3$ & 175 \\
\hline $\mathrm{c}$ & $1 / 2$ & $1 / 3$ & 0 & 0 & 0 & 0 & 1 & 0 & $+2 / 3$ & 1350 \\
\hline $\mathrm{b}$ & $1 / 2$ & $1 / 3$ & 0 & 0 & 0 & 0 & 0 & -1 & $-1 / 3$ & 4500 \\
\hline $\mathrm{t}$ & $1 / 2$ & $1 / 3$ & 0 & 0 & 0 & 0 & 0 & 0 & $+2 / 3$ & 173000 \\
\hline$\overline{\mathrm{e}^{-}}$ & $1 / 2$ & 0 & 1 & 0 & 0 & 0 & 0 & 0 & -1 & 0.511 \\
\hline$\mu^{-}$ & $1 / 2$ & 0 & 1 & 0 & 0 & 0 & 0 & 0 & -1 & 105.658 \\
\hline$\tau^{-}$ & $1 / 2$ & 0 & 1 & 0 & 0 & 0 & 0 & 0 & -1 & 1777.1 \\
\hline$\nu_{\mathrm{e}}$ & $1 / 2$ & 0 & 1 & 0 & 0 & 0 & 0 & 0 & 0 & $0(?)$ \\
\hline$\nu_{\mu}$ & $1 / 2$ & 0 & 1 & 0 & 0 & 0 & 0 & 0 & 0 & $0(?)$ \\
\hline$\nu_{\tau}$ & $1 / 2$ & 0 & 1 & 0 & 0 & 0 & 0 & 0 & 0 & $0(?)$ \\
\hline$\gamma$ & 1 & 0 & 0 & 0 & 0 & 0 & 0 & 0 & 0 & 0 \\
\hline gluon & 1 & 0 & 0 & 0 & 0 & 0 & 0 & 0 & 0 & 0 \\
\hline$W^{ \pm}$ & 1 & 0 & 0 & 0 & 0 & 0 & 0 & 0 & $\mp$ & 80220 \\
\hline $\mathrm{Z}$ & 1 & 0 & 0 & 0 & 0 & 0 & 0 & 0 & 0 & 91187 \\
\hline
\end{tabular}

Table 2.1: List of known (discovered) Standard Model elementary particles and some of their properties. There are 12 fermionic particles (first two raws) and five bosonic particles (last raw). Postulated but not yet discovered bosons are graviton and Higgs boson.

\begin{tabular}{||c|c|c|c|c||}
\hline & leptons & quarks & antileptons & antiquarks \\
\hline 1st generation & $\mathrm{e}^{-}$ & $\mathrm{d}$ & $\mathrm{e}^{+}$ & $\overline{\mathrm{d}}$ \\
& $\nu_{\mathrm{e}}$ & $\mathrm{u}$ & $\bar{\nu}_{\mathrm{e}}$ & $\overline{\mathrm{u}}$ \\
\hline 2nd generation & $\mu^{-}$ & $\mathrm{s}$ & $\mu^{+}$ & $\overline{\mathrm{s}}$ \\
& $\nu_{\mu}$ & $\mathrm{c}$ & $\bar{\nu}_{\mu}$ & $\overline{\mathrm{c}}$ \\
\hline \multirow{3}{*}{ 3rd generation } & $\tau^{-}$ & $\mathrm{b}$ & $\tau^{+}$ & $\overline{\mathrm{b}}$ \\
& $\nu_{\tau}$ & $\mathrm{t}$ & $\bar{\nu}_{\tau}$ & $\overline{\mathrm{t}}$ \\
\hline
\end{tabular}

Table 2.2: Fermions can be divided in three generations, each consisting of a lepton, associated neutrino, and two quarks.

force does not decrease with distance. The potential energy will become high enough to create a quark-antiquark pair when it is tried to disjoin an (anti)quark from a hadron. This will result in two hadrons and not in free quarks. The composition of (anti)quarks 
Chapter 2. Theory

\begin{tabular}{||l|cl||}
\hline$\pi^{0}$ & $\frac{1}{2} \sqrt{2}(\mathrm{u} \overline{\mathrm{u}}+\mathrm{d} \overline{\mathrm{d}})$ & 134.9764 \\
$\pi^{+}$ & $\mathrm{ud}$ & 139.56995 \\
$\eta$ & $\frac{1}{\sqrt{6}}(\mathrm{u} \overline{\mathrm{u}}+\mathrm{d} \overline{\mathrm{d}}-2 \mathrm{~s} \overline{\mathrm{s}})$ & 547.30 \\
$\eta^{\prime}$ & $\frac{1}{\sqrt{3}}(\mathrm{u} \overline{\mathrm{u}}+\mathrm{d} \overline{\mathrm{d}}+\mathrm{s} \overline{\mathrm{s}})$ & 958.78 \\
$\mathrm{~K}^{0}$ & $\mathrm{~s} \overline{\mathrm{d}}$ & 497.672 \\
$\mathrm{~K}^{+}$ & $\mathrm{u} \overline{\mathrm{s}}$ & 493.677 \\
$\mathrm{D}^{0}$ & $\mathrm{c} \overline{\mathrm{u}}$ & 1864.6 \\
$\mathrm{D}^{+}$ & $\mathrm{c} \overline{\mathrm{d}}$ & 1869.4 \\
$\mathrm{~B}^{0}$ & $\mathrm{~d} \overline{\mathrm{b}}$ & 5279.4 \\
$\mathrm{~B}^{+}$ & $\mathrm{u} \overline{\mathrm{b}}$ & 5279.1 \\
$\mathrm{~J} / \Psi$ & $\mathrm{c} \overline{\mathrm{c}}$ & 3096.8 \\
$\Upsilon$ & $\mathrm{b} \overline{\mathrm{b}}$ & 9460.37 \\
$\mathrm{~F}^{+}$ & $\mathrm{c} \overline{\mathrm{s}}$ & 1969.0 \\
\hline
\end{tabular}

Table 2.3: List of mesons, their quark content and mass in $\mathrm{MeV}$.

of the hadrons is given in tables 2.4 and ??, together with their mass in $\mathrm{MeV}$ in their ground state: 
Chapter 2. Theory

\begin{tabular}{||l|ll||}
\hline $\mathrm{p}^{+}$ & $\mathrm{u} \mathrm{u} \mathrm{d}$ & 938.27231 \\
$\mathrm{p}^{-}$ & $\overline{\mathrm{u}} \overline{\mathrm{u}} \overline{\mathrm{d}}$ & 938.27231 \\
$\mathrm{n}^{0}$ & $\mathrm{u} \mathrm{d} \mathrm{d}$ & 939.56563 \\
$\overline{\mathrm{n}}^{0}$ & $\overline{\mathrm{u}} \overline{\mathrm{d}} \overline{\mathrm{d}}$ & 939.56563 \\
$\Lambda$ & $\mathrm{u} \mathrm{d} \mathrm{s}$ & 1115.684 \\
$\bar{\Lambda}$ & $\overline{\mathrm{u}} \overline{\mathrm{d}} \overline{\mathrm{s}}$ & 1115.684 \\
$\Sigma^{+}$ & $\mathrm{u} \mathrm{u} \mathrm{s}$ & 1189.37 \\
$\overline{\Sigma^{+}}$ & $\overline{\mathrm{d}} \overline{\mathrm{d}} \overline{\mathrm{s}}$ & 1197.436 \\
$\Sigma^{0}$ & $\mathrm{u} \mathrm{d} \mathrm{s}$ & 1192.55 \\
$\overline{\Sigma^{0}}$ & $\overline{\mathrm{u}} \overline{\mathrm{d}} \overline{\mathrm{s}}$ & 1192.55 \\
$\Sigma^{-}$ & $\mathrm{d} \mathrm{d} \mathrm{s}$ & 1197.436 \\
$\overline{\Sigma^{-}}$ & $\overline{\mathrm{u}} \overline{\mathrm{u}} \overline{\mathrm{s}}$ & 1189.37 \\
$\Xi^{0}$ & $\mathrm{u} \mathrm{s} \mathrm{s}$ & 1314.9 \\
$\bar{\Xi}^{0}$ & $\overline{\mathrm{u}} \overline{\mathrm{s}} \overline{\mathrm{s}}$ & 1314.9 \\
$\Xi^{-}$ & $\mathrm{d} \mathrm{s} \mathrm{s}$ & 1321.32 \\
$\Xi^{+}$ & $\overline{\mathrm{d}} \overline{\mathrm{s}} \overline{\mathrm{s}}$ & 1321.32 \\
$\Omega^{-}$ & $\mathrm{s} \mathrm{s} \mathrm{s}$ & 1672.45 \\
$\Omega^{+}$ & $\overline{\mathrm{s}} \overline{\mathrm{s}} \overline{\mathrm{s}}$ & 1672.45 \\
$\Lambda_{c}^{+}$ & $\mathrm{u} \mathrm{d} \mathrm{c}$ & 2285.1 \\
$\Delta^{2-}$ & $\overline{\mathrm{u}} \overline{\mathrm{u}} \overline{\mathrm{u}}$ & 1232.0 \\
$\Delta^{2+}$ & $\mathrm{u} \mathrm{\textrm {u } \mathrm { u }}$ & 1232.0 \\
$\Delta^{+}$ & $\mathrm{u} \mathrm{u} \mathrm{d}$ & 1232.0 \\
$\Delta^{0}$ & $\mathrm{u} \mathrm{d} \mathrm{d}$ & 1232.0 \\
$\Delta^{-}$ & $\mathrm{d} \mathrm{d} \mathrm{d}$ & 1232.0 \\
\hline
\end{tabular}

Table 2.4: List of baryons, their quark content and mass in $\mathrm{MeV}$.

Each quark exists in one of the two spin $\frac{1}{2}$ states. So mesons are bosons with spin 0 or 1 in their ground state, while baryons are fermions with spin $\frac{1}{2} \frac{3}{2}$ in the ground state. There exist excited states (resonances) with higher internal angular momentum quantum number $L$. Neutrino's have a helicity of $-\frac{1}{2}$ while antineutrinos have only $+\frac{1}{2}$ as possible value.

If a physical system remains unchanged after some transformation then the transformation is called symmetry transformation or simply symmetry. For every such transformation we may define an unitary operator $U$ in Hilbert space. This operator 


\section{Chapter 2. Theory}

commutes with the Hamiltonian of the system. Set of symmetry transformations has certain properties that define it as group. The operators corresponding to these symmetry transformations mirror its group structure. The quantum numbers are subject to conservation laws. These can be derived from symmetries of physical system: continuous symmetries give rise to additive conservation laws, discrete symmetries result in multiplicative conservation laws.

Geometrical conservation laws are invariant under Lorentz transformations and the CPT-operation. These are:

1. Mass/energy because the laws of nature are invariant for translations in time.

2. Momentum because the laws of nature are invariant for translations in space.

3. Angular momentum because the laws of nature are invariant for rotations.

Dynamical conservation laws are invariant under the CPT-operation. These are:

1. Electrical charge because the Maxwell equations are invariant under gauge transformations.

2. Color charge is conserved.

3. Baryon number and lepton number are conserved but not under a possible SU(5) symmetry of the laws of nature.

4. Quark's color is only conserved under the color interaction.

5. Parity is conserved except for weak interactions.

Symmetries giving rise to the internal quantum numbers, e.g. weak isospin and color, are called internal symmetries. Internal symmetries that depend of time and space are called local gauge symmetries. 
Interactions between fermions are mediated by gauge bosons. There are four fundamental forces in the nature: strong, electromagnetic, weak and gravitational, listed in the decreasing order of strength which spans 38 orders of magnitude. All particles feel week force which is carried by charged $W^{ \pm}$or neutral $Z^{\circ}$ bosons. Charged particles feel electromagnetic force, mediated by electrically neutral and massless photons. Only quarks feel the strongest of all forces, the strong force, which mediated by one of eight electrically neutral, massless and colorful gluons. The weakest of the four forces, gravity, couples to all particles and is postulated to be mediated by a massless spin two boson, gravitino. As of present, gravitino is yet to be observed. The first three forces are encompassed by the SM, while gravity remains outside. The interactions between the elementary particles in SM is based on local gauge symmetries, whose operators form a group. If the system is represented by a field $\psi$ then the presence of each local gauge symmetry is represented by gauge field $\alpha$. In the simplest case of $U(1)$ symmetry group, the symmetry transformation $\psi \rightarrow e^{i \alpha(x) \psi}$, is mediated by only one gauge boson field.

\subsection{The standard model}

The SM is a field theory based on gauge symmetry group that is a direct product of three gauge symmetry groups $\mathrm{SU}(3)_{C} \otimes \mathrm{SU}(2)_{T} \otimes \mathrm{U}(1)_{Y}$. The $\mathrm{SU}(3)_{C}$ symmetry group is used to formulate strong force in a theory known as Quantum Chromodynaics (QCD). The composed symmetry group $\mathrm{SU}(2)_{T} \otimes \mathrm{U}(1)_{Y}$ and the Electroweak Theory (EWT) describes both electromagnetic and weak forces. Electromagnetic interactions are described in a local gauge theory called Quantum Electrodynamics (QED) which is based on $\mathrm{U}(1)_{e m}$ symmetry group. The subscripts $C, L$, and $Y$ refer to the quantum numbers preserved by the symmetries: color charge, weak isospin, and hypercharge. The number of generators in a $N$-dimensional special unitary group $\mathrm{SU}(\mathrm{N})$ is $N^{2}-1$. To 
understand the SM one needs to understand basic principle behind gauge theories.

\subsubsection{Gauge field theories}

A physical theory is usually described by the Lagrangian of the system which is invariant under certain transformations (symmetries). When these transformations are performed equally for any space-time point, then we say that the system has global symmetry. If, on the other hand, the Lagrangian is invariant under some transformation performed only on a localized region of space-time without affecting what happens in another region, then we say that the system has local or gauge symmetries.

It is important to understand how local gaguge symmetry leads to interaction of fields which were originally non-interacting. For sake of illustration, we look at a simply case of classical scalar fields.

\section{Example : ScalarO(n)gaugetheory}

Consider a set of $n$ non-interacting real scalar fields. The Lagrangian density of the system is:

$$
\mathcal{L}=\frac{1}{2} \sum_{i}\left(\partial_{\mu} \phi_{i}\right)^{T} \partial^{\mu} \phi_{i}-\frac{1}{2} m^{2} \sum_{i} \phi_{i}^{2}
$$

where $i$ runs over the number of fields. Or more compactly,

$$
\mathcal{L}=\frac{1}{2}\left(\partial_{\mu} \Phi\right)^{T} \partial^{\mu} \Phi-\frac{1}{2} m^{2} \Phi^{2}
$$

by introducing a vector of fields $\Phi=\left(\phi_{1}, \phi_{2}, . ., \phi_{n}\right)^{T}$. The Lagrangian is invariant under the transformation

$$
\Phi \rightarrow G \Phi
$$




\section{Chapter 2. Theory}

for any $G$ a constant matrix belonging to the $n$-by-n orthogonal group $\mathrm{O}(\mathrm{n})$. Since $G$ is constant, this is a global symmetry. Nother'stheorem (ref) implies conservation of the current

$$
J_{\mu}^{a}=i \partial_{\mu} \Phi^{T} T^{a} \Phi
$$

where $T^{a}$ are generators of the $\mathrm{SO}(\mathrm{n})$ group. If we, however, demand that the above Lagrangian has local symmetry, then $G$ matrices must be functions of time-space coordinates $x, G=G(x)$. We immediately run into a problem: $G(x)$ matrices do not pass through the derivatives:

$$
\partial_{\mu}(G \Phi)^{T} \partial^{\mu} G \Phi \neq \partial_{\mu} \Phi^{T} \partial^{\mu} \Phi
$$

But we then define a derivative that is a function of space-time, a covariant derivative

$$
D_{\mu} \equiv \partial_{\mu}+g A_{\mu}(x)
$$

where $A_{\mu}$ is a gauge - field that has the following transformation properties

$$
A_{\mu} \rightarrow G(x) A_{m u} G^{-1}(x)-\frac{1}{g} \partial_{\mu} G(x) G^{-1}(x)
$$

and $g$ is a constant, quantity defining the strength of an interaction, i.e., a coupling constant. The gauge field is an element of the Lie algebra of the local symmetry group. It can be expressed as

$$
A_{\mu}=A_{\mu a} T^{a}
$$

There are as many gauge fields as there are Lie algebra generator. 


\section{Chapter 2. Theory}

If we use the covariant derivative in the equation 2.1 we obtain a locally gauge invariant Lagrangian

$$
\mathcal{L}_{l o c}=\frac{1}{2}\left(D_{\mu} \Phi\right)^{T} D^{\mu} \Phi-\frac{1}{2} m^{2} \Phi^{T} \Phi
$$

The difference between this lagrangian and the original globally symmetric one is the interaction Lagrangian

$$
\mathcal{L}_{\text {int }}=\frac{g}{2} \Phi^{T} A_{\mu}^{T} \partial^{\mu} \Phi+\frac{g}{2}\left(\partial_{\mu} \Phi\right)^{T} A^{\mu} \Phi
$$

This term introduces the interaction between the scalar fields just because we required local symmetry of the system. Notice that to define the covariant derivative $D$ we must set know the values of the gauge field $A(x)$ for all $x$. The values of this field can be given by solving a field equation. Further, we require that the Lagrangian responsible for generating this field equation $\left(L_{g f}\right)$ is locally gauge invariant as well. One possible form for the gauge field Lagrangian is

$$
\mathcal{L}_{g f}=-\frac{1}{4} \operatorname{Tr}\left(F^{\mu \nu} F_{\mu \nu}\right)
$$

with

$$
F_{\mu \nu}=\left[D_{\mu}, D^{\nu}\right]
$$

This is called Yang-Mills action.

So the complete Lagrangian for for the $\mathrm{O}(\mathrm{n})$ gauge theory is

$$
\mathcal{L}=\mathcal{L}_{\text {loc }}+\mathcal{L}_{g f}=\mathcal{L}_{\text {global }}+\mathcal{L}_{\text {int }}+\mathcal{L}_{g f}
$$

Now, that the basic principles of gauge theories is presented, we can now understand quantum field gauge theories. Before local symmetries can be quantized one must 


\section{Chapter 2. Theory}

restrict them or perform gaugefixing, which is discussed elsewhere (Sakurai's Advnace Quantum Mechanics). The simplest quantum field gauge theory is the Theory of Quantum Electrodynamics (QED).

\subsubsection{Quantum electrodynamics}

The QED is the simples and the most successful gauge theory, predicting experimental results to many orders of magnitude. In the QED Lagrangian fermions with mass $m$ and electric charge $Q$ are represented as Dirac fields $\psi(x)$ :

$$
\mathcal{L}=\bar{\psi}(x)\left(i \partial_{\mu} \gamma^{\mu}-m\right) \psi(x)
$$

From this one can obtain the equation of motion (Dirac equation)

$$
\left(i \partial_{\mu} \gamma^{\mu}-m\right) \psi=0
$$

It is easy to see that the Lagrangian in 2.14 has a global symmetry for $\psi \rightarrow e^{i \theta} \psi$. The gauge group here is $\mathrm{U}(1)$, which consist of one generator, just the phase angle of the field. One can show that if we make a local transformation $\psi \rightarrow e^{i Q \alpha(x)} \psi$ then the locally invariant Lagrangian becomes

$$
\mathcal{L}_{Q E D}=\bar{\psi}(x)\left(i \gamma^{\mu} D_{\mu}-m\right) \psi(x)-\frac{1}{4} F^{\mu \nu} F_{\mu \nu}
$$

where the normal derivative $\partial_{\mu}$ is replaced by covariant derivative $D_{\mu}$,

$$
D_{\mu} \equiv\left(\partial_{\mu}-i q A_{\mu}(x)\right.
$$

This defines a single gauge (boson) field $A$, the photon field. The field strength tensor is

$$
F_{\mu \nu} \equiv \partial_{\mu} A^{\nu}-\partial^{\nu} A^{\mu}
$$


Chapter 2. Theory

The interaction Lagrangian is given by

$$
\mathcal{L}_{i n t_{Q E D}}=\bar{\psi}(x) \gamma^{\mu} \psi(x) A_{\nu}(x)=J^{\nu}(x) A_{\nu}(x)
$$

where $J(x)$ is the electromagnetic current density which is conserved. The charge, $Q$ is given by

$$
q=\int J_{0}(x) d^{3} x
$$

\subsubsection{The electroweak theory}

The electroweak interaction arises from the necessity to keep the Lagrange density invariant for local gauge transformations of the group $\mathrm{SU}(2) \otimes \mathrm{U}(1)$. Right- and lefthanded spin states are treated different because the weak interaction does not conserve parity. If a fifth Dirac matrix is defined by:

$$
\gamma_{5}:=\gamma_{1} \gamma_{2} \gamma_{3} \gamma_{4}=-\left(\begin{array}{cccc}
0 & 0 & 1 & 0 \\
0 & 0 & 0 & 1 \\
1 & 0 & 0 & 0 \\
0 & 1 & 0 & 0
\end{array}\right)
$$

the left- and right- handed solutions of the Dirac equation for neutrino's are given by:

$$
\psi_{\mathrm{L}}=\frac{1}{2}\left(1+\gamma_{5}\right) \psi \quad \text { and } \quad \psi_{\mathrm{R}}=\frac{1}{2}\left(1-\gamma_{5}\right) \psi
$$

It appears that neutrino's are always left-handed while antineutrinos are always righthanded.

For hypercharge $Y$, charge $Q$, weak isospin $T_{3}$, is defined

$$
Q=\frac{1}{2} Y+T_{3}
$$


Chapter 2. Theory

so $\left[Y, T_{k}\right]=0$. The group $\mathrm{U}(1)_{Y} \otimes \mathrm{SU}(2)_{T}$ is taken as symmetry group for the electroweak interaction because the generators of this group commute. The multiplets are classified as follows:

\begin{tabular}{|c|c|c|c|c|c|}
\hline & $\mathrm{e}_{\mathrm{R}}^{-}$ & $\nu_{\mathrm{eL}} \mathrm{e}_{\mathrm{L}}^{-}$ & $\mathrm{u}_{\mathrm{L}} \quad \mathrm{d}_{\mathrm{L}}^{\prime}$ & $\mathrm{u}_{\mathrm{R}}$ & $\mathrm{d}_{\mathrm{R}}$ \\
\hline$T$ & 0 & $\frac{1}{2}$ & $\frac{1}{2}$ & 0 & 0 \\
\hline$T_{3}$ & 0 & $\frac{1}{2}-\frac{1}{2}$ & $\frac{1}{2}-\frac{1}{2}$ & 0 & 0 \\
\hline$Y$ & -2 & -1 & $\frac{1}{3}$ & $\frac{4}{3}$ & $-\frac{2}{3}$ \\
\hline
\end{tabular}

Now, 1 field $B_{\mu}(x)$ is connected with gauge group $\mathrm{U}(1)$ and 3 gauge fields $\vec{A}_{\mu}(x)$ are connected with SU(2). The total Lagrange density (minus the field terms) for the electron-fermion field now becomes:

$$
\begin{aligned}
\mathcal{L}_{0, \mathrm{EW}}= & -\left(\overline{\psi_{\nu \mathrm{e}, \mathrm{L}}}, \overline{\psi_{\mathrm{eL}}}\right) \gamma^{\mu}\left(\partial_{\mu}-i \frac{g}{\hbar} \vec{A}_{\mu} \cdot\left(\frac{1}{2} \vec{\sigma}\right)-\frac{1}{2} i \frac{g^{\prime}}{\hbar} B_{\mu} \cdot(-1)\right)\left(\begin{array}{c}
\psi_{\nu \mathrm{e}, \mathrm{L}} \\
\psi_{\mathrm{eL}}
\end{array}\right)- \\
& \overline{\psi_{\mathrm{eR}}} \gamma^{\mu}\left(\partial_{\mu}-\frac{1}{2} i \frac{g^{\prime}}{\hbar}(-2) B_{\mu}\right) \psi_{\mathrm{eR}}
\end{aligned}
$$

Here, $\frac{1}{2} \vec{\sigma}$ are the generators of $T$ and -1 and -2 the generators of $Y$.

\subsubsection{Spontaneous symmetry breaking: the Higgs mechanism}

All leptons are massless in the equations above. Their mass is generated by spontaneous symmetry breaking. This means that the dynamic equations which describe the system have a symmetry which the ground state does not have. It is assumed that there exists a weak isospin-doublet of scalar fields $\Phi$ with electrical charges +1 and 0 and potential $V(\Phi)=-\mu^{2} \Phi^{*} \Phi+\lambda\left(\Phi^{*} \Phi\right)^{2}$. Their antiparticles have charges -1 and 0 . The extra terms in $\mathcal{L}$ arising from these fields, $\mathcal{L}_{H}=\left(D_{L \mu} \Phi\right)^{*}\left(D_{L}^{\mu} \Phi\right)-V(\Phi)$, are globally $\mathrm{U}(1) \otimes \mathrm{SU}(2)$ symmetric. Hence the state with the lowest energy corresponds with the 


\section{Chapter 2. Theory}

state $\Phi^{*}(x) \Phi(x)=v=\mu^{2} / 2 \lambda=$ constant. The field can be written (were $\omega^{ \pm}$and $z$ are Nambu-Goldstone bosons which can be transformed away, and $m_{\phi}=\mu \sqrt{2}$ ) as:

$$
\Phi=\left(\begin{array}{c}
\Phi^{+} \\
\Phi^{0}
\end{array}\right)=\left(\begin{array}{c}
i \omega^{+} \\
(v+\phi-i z) / \sqrt{2}
\end{array}\right) \text { and }\langle 0|\Phi| 0\rangle=\left(\begin{array}{c}
0 \\
v / \sqrt{2}
\end{array}\right)
$$

Because this expectation value $\neq 0$ the EWK symmetry is broken, $S U(2) \otimes S U_{Y}(1) \rightarrow$ $S U_{E M}(1)$. When the gauge fields in the resulting Lagrange density are separated one obtains:

$$
\begin{aligned}
W_{\mu}^{-} & =\frac{1}{2} \sqrt{2}\left(A_{\mu}^{1}+i A_{\mu}^{2}\right), \quad W_{\mu}^{+}=\frac{1}{2} \sqrt{2}\left(A_{\mu}^{1}-i A_{\mu}^{2}\right) \\
Z_{\mu} & =\frac{g A_{\mu}^{3}-g^{\prime} B_{\mu}}{\sqrt{g^{2}+g^{\prime 2}}} \equiv A_{\mu}^{3} \cos \left(\theta_{\mathrm{W}}\right)-B_{\mu} \sin \left(\theta_{\mathrm{W}}\right) \\
A_{\mu} & =\frac{g^{\prime} A_{\mu}^{3}+g B_{\mu}}{\sqrt{g^{2}+g^{\prime 2}}} \equiv A_{\mu}^{3} \sin \left(\theta_{\mathrm{W}}\right)+B_{\mu} \cos \left(\theta_{\mathrm{W}}\right)
\end{aligned}
$$

where $\theta_{\mathrm{W}}$ is called the Weinberg angle. For this angle holds: $\sin ^{2}\left(\theta_{\mathrm{W}}\right)=0.255 \pm$ 0.010. Relations for the masses of the field quanta can be obtained from the remaining terms: $M_{W}=\frac{1}{2} v g$ and $M_{Z}=\frac{1}{2} v \sqrt{g^{2}+g^{\prime 2}}$, and for the elementary charge holds: $e=\frac{g g^{\prime}}{\sqrt{g^{2}+g^{\prime 2}}}=g^{\prime} \cos \left(\theta_{\mathrm{W}}\right)=g \sin \left(\theta_{\mathrm{W}}\right)$

Experimentally it is found that $M_{W}=80.022 \pm 0.26 \mathrm{GeV} / \mathrm{c}^{2}$ and $M_{Z}=91.187 \pm$ $0.007 \mathrm{GeV} / \mathrm{c}^{2}$. According to the weak theory this should be: $M_{W}=83.0 \pm 0.24 \mathrm{GeV} / \mathrm{c}^{2}$ and $M_{Z}=93.8 \pm 2.0 \mathrm{GeV} / \mathrm{c}^{2}$.

\subsubsection{Quantum chromodynamics}

Colored particles interact because the Lagrange density is invariant for the transformations of the group $\mathrm{SU}(3)$ of the color interaction. A distinction can be made between two types of particles: 


\section{Chapter 2. Theory}

1. "White" particles: they have no color charge, the generator $\vec{T}=0$.

2. "Colored" particles: the generators $\vec{T}$ are $83 \times 3$ matrices. There exist three colors and three anticolors.

The Lagrange density for colored particles is given by

$$
\mathcal{L}_{\mathrm{QCD}}=i \sum_{k} \overline{\Psi_{k}} \gamma^{\mu} D_{\mu} \Psi_{k}-\frac{1}{4} F_{\mu \nu}^{a} F_{a}^{\mu \nu}
$$

The gluons remain massless. The mass is further obtained from Higgs coupling, and symmetry $S U_{C}(3)$ is unbroken.

The SM has been very successful at predicting the results of an enormous number of experiments with incredible precision for the past two decades. The electroweak observables as predicted by the SM are all substantially within the exprimental uncertainties [?]. The flavor missing as described by the CKM matrix is also well within the theory [?]. The perturbative QCD precisions successfully lie within the experimental results. Some of the most important QCD achievements were corrections to the electroweak observables.. The non-perturbative QCD calculations, which are notoriously difficult, have even recently made successful mass and decay-constant predictions by solving QCD field equations numerically on the space-time lattice. There are however some experimental observations that are not explained by the SM, such as neuturino oscillations and the dark matter in the Universe. For example, because the neutrinos do undergo flavor changes (oscillations), it is certain that neutrinos have mass.

\subsubsection{Beyond SM}

The SM of the particle physics, albeit, extremely successful phenomenologically, has been regarded only as a low energy approximation of a more fundamental theory. Few of the reasons that lead us to believe so are listed below. 
- The SM quantum numbers listed in table 2.1 are, to say the least, looking bizarre, with the hypercharge weirdest of all. The assignments of these are crucial and necessary to rid of anomalies which would jeopardize the gauge invariance at the quantum level, which would otherwise make the theory inconsistent. Another bizarre detail is why are the hypercharges given in units of $1 / 6$. In principle, hypercharges can be any numbers, even irrational. However, these quantized hypercharges are necessary to obtain neutrality of bulk matter in the universe $Q(e)+2 Q(u)+Q(d)=0$ to one part in $10^{21}$.

- The most essential ingredient of SM, the EWK symmetry breaking, probably seem the ugliest to most people. The spin one gauge bosons grouped in multiplets are natural consequences of the gauge theories. However, there is only one spinless multiplet in the SM, the Higgs doublet, which condenses in the vacuum due to the Mexican-hat potential. There, the potential has to be arranged in a way to break the symmetry without any microscopic explanations. Higgs doublet is introduced ad hoc just for the purpose of EWK symmetry breaking and to further give masses to quarks and leptons.

- The gauge hierarchy problem in SM arises from the fact that corrections to Higgs boson mass are proportional to the mass-squared of the particle to which Higgs boson couples. If there is such a particle with the mass much higher than Higgs' (EWK scale), on the order of GUT or Plank scale, then the correction to the Higgs mass would have to cancel with a wonderful precision of $m_{E W}^{2} / m_{G U T}^{2} \approx 10^{-30}$ [8], [9]. Yukawa hierarchy problem in SM refers to unnatural mass difference between the particles. Given that neutrinos are believed to have mass, these differences span over 13 orders of magnitude.

- Why are there three generations? Why do fermions have masses that stretch over thirteen orders of magnitude, between the neutrinos and the top quark. We have no concrete understanding of the mass spectrum nor the mixing patterns. 
- The SM is notorious for its problems of infinities. Probably the most serious one is zero point energy, $H_{0}$, which is given, modulo some constants, as

$$
H_{0}=\int d^{3} k\left(\sum_{i=1, b} k_{0 i}-\sum_{i=1, f} k_{0 i}\right)
$$

where $k_{0}=\sqrt{\vec{k}^{2}+m^{2}}$ where $i$ goes over all boson in the first sum and over all fermion fields in the second sum. Note that $H_{0}$ is quartic in $k$ whose integration upper limit is infinity. Usually, one can redefine a constant zero point energy by setting it to zero, but in this case it remains utterly puzzling what to do with infinity to the fourth power.

- After many astronomical observations suggest that SM particles comprise only a small fraction of the entire matter in the universe. Namely, matter contributes only $30 \%$ to the total energy density of the universe, and baryons contribute only $5 \%$. The rest $25 \%$ is not visable. That is to say, it does not couple to photons.

All the reasons listed above ask for a more fundamental theory underlying the SM. History suggests that more fundamental theories always lie on distances shorter than the distance scale of the problem. For example, Mendeleev periodic table was understood in terms of the bound electronic states in the atoms, Pauli exclusion principle and spin. The description of ideal gas was facilitated by statistical mechanics of free molecules. The deviation from ideal gas, described by the van der Waals equation, is a consequence of the finite size of the molecules and their interactions. The list can go on. The SM distance scale is given by the size of Higgs boson condensate, which is on the order of $v=10^{2} \mathrm{GeV}$, which in natural units is translated to $d \approx 10-16 \mathrm{~cm}$. The idea is that the SM is valid to some distances shorter than $d$, but then for even shorter distances new physics will appear which will take over the SM.

A number of theoretical motivations together with the effort to describe the experimental findings described above: inspired new ideas, like the following: grand 


\section{Chapter 2. Theory}

unification, Yukawa or gauge hierarchy problems, extra dimensions, formulations of particles as strings, dynamic symmetry breaking, quark and lepton compositness, supersymmetry. I list major outlines of these ideas below, and give special treatment to SUSY in the following subsection. The most attractive SUSY models encompass most of the above ideas.

Grand unification theories (GUT) attempt to unify strong and electroweak interactions into one. In this case the SM particles are representation of a single gauge symmetry group. At some energy scale the symmetry is thought to be broken giving rise to the $\mathrm{SU}(3)_{C} \otimes \mathrm{SU}(2)_{T} \otimes \mathrm{U}(1)_{Y}$. GUTs are mainly motivated by the fact that three coupling constants (strong, weak, electromagnetic) seem to converge at one point on energy scale, around $10^{16} \mathrm{GeV}$. GUT calls for violation of letpon and baryon number, and as a consequence, decay of proton.

The extra dimensions theories call for a universe of $4+N$ dimensions, of which the four are the time-space ones we live in, and the extra $N$ dimensions are compact. These theories solve the gauge hierarchy problem by definition, since the Plank scale is as low as $1 \mathrm{TeV}$ due to extra dimensions.

The models with quark and lepton compositness assume that SM fermions are not elementary, but made of constituents. The large binding energy and the chiral nature of the interactions between constituents would explain masses of leptons and quarks. These models naturally imply existence of fermionic excited states. Up to date, there is no evidence of such.

Dynamic symmetry breaking models propose to substitute Higss symmetry breaking mechanism. Instead of Higgs field acquiring mass from its non-zero vacuum expectation value $(v e v)$, it is proposed that a fermion bilinear (i.e., a fermion-antifermion pair) gains a vev. In these models it is possible to describe masses of all fermions and $W$ and $Z$ bosons. 
Chapter 2. Theory

In string theories, particles are described as extended objects, one dimensional strings. String theories that include fermions and bosons are supersymmetric by definition. They are formulated in more than 4-dimensional time-space. One of the theoretically most appealing, $\mathrm{M}$ theory, is defined in 11 dimensions. $\mathrm{M}$ theory also provides for unification of gravity, electroweak, and strong force in terms of quantized gauge field theory (ref sc 34-35).

\subsection{Supersymmetry}

Concepts of supersymmetry (SUSY) and the most popular phenomenological models are described in this section. Properties of SUSY and the way it solves some of the theoretical challenges are discussed first. The minimal supersymmetric extension to the SM (MSSM) is described next. The problem of a large number of parameter in MSSM and the possible solutions to it are discussed as well. The section is concluded with the overview of benchmark models sensitive to the present analysis, such as mSUGRA and its extension with non-universality of scalar masses.

Supersymmetry is the symmetry between boson and fermion. Mathematically, supersymmetry arises from the requirement that the theory should be invariant under transformations that convert bosons to fermions and vice versa. Such a theory might seam strange at first, since it changes particle statistics (Fermi-Dirac and BoseEinstein) and this is why it is called supersymmetry. If one can imagine that God created two types of particles, one can imagine that She created a way of converting one into another. A supersymmetric theory has the same number of bosonic and fermionic degrees of freedom, that is every particle has its corresponding superpartner. The names of the superpartners to bosons have a suffix "ino" (e.g. gluino), and superpartners to fermions prefix "s" (e.g. squark). The supersymmetric transformations are global transformations of the fields are an extension to the Poincare group 
Chapter 2. Theory

transformations [?].

SUSY is very appealing theoretically. The very first reason why Supersymmetry attracts so much attention is that it ameliorates the zero point energy problem of the SM. Recall the equation 2.21 for the zero point energy in the SM. It is useful to rewrite it as

$$
H_{0}=\int d^{3} k k\left(\sum_{i=1, b} \sqrt{1+\frac{m_{i}^{2}}{k^{2}}}-\sum_{i=1, f} \sqrt{1+\frac{m_{i}^{2}}{k^{2}}}\right)
$$

In case number of boson fields equals number of fermion fields in nature, the first term in equation 2.22 equation, which is quartic in $k$, cancels. It cancels regardless if bosons and fermions are degenerate in mass. However, the second term which is only logarithmic in $k$ only cancels in case of mass degeneracy between bosons and fermions. This was first realized by Wess and Zumino in 1970's, at the dawn of Supersymmetry [6].

It naturally solves the gauge hierarchy problem. The consistent string theories require SUSY, the SM gauge coupling unification is achievable (SUSY GUTs). Then from the experimental point of view, it can possibly explain the neutrino masses. SUSY is one of the major candidates for explanation of dark matter in the universe.

The gauge hierarchy problem is solved in the following way: the correction to the Higgs mass by the virtual fermions and bosons is expressed as a self-energy diagrams shown in Figure 2.1. The leading terms in the mass correction calculation are of opposite sign, one of fermion and one of boson. So, in the case of mass degeneracy of fermions and bosons, the quadratic divergence in the calculation cancels.

Since the SUSY particles are not mass degenerate with their SM partners (we would have seen them by now), then it means that SUSY extension of SM must have supersymmetry broken at some energy scale down the line. The ways of SUSY breaking are not known so there are various models that describe it. The common way to extend 

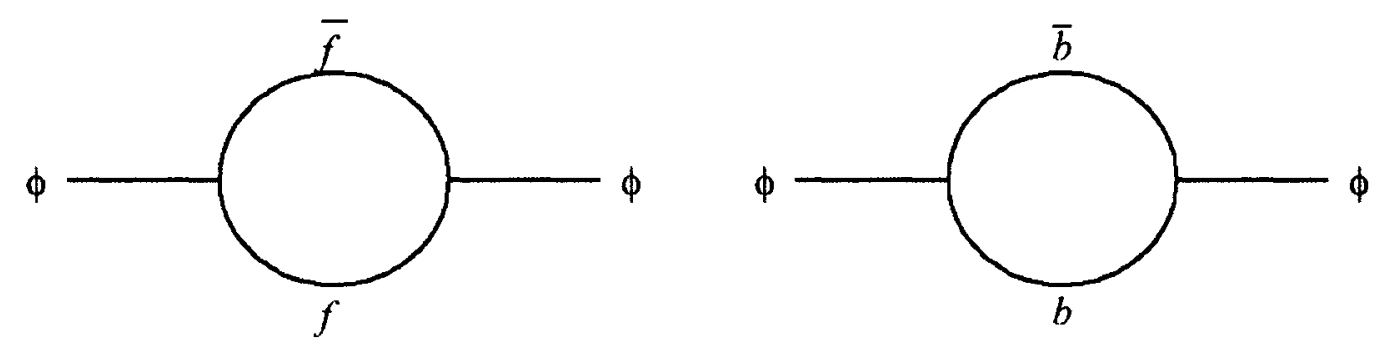

Figure 2.1: Feynman diagrams for fermionic (left) and bosonic (right) contributions to the Higgs's self energy. If the same diagrams exist for both bosons and fermions of exactly the same mass, they cancel exactly

the SM is phenomenological: the supersymmetrized SM Lagrangian prior to the EWK symmetry breaking (EWSB) is extended by the EWK and SUSY breaking terms.

\section{The Minimal Supersymmetric Standard Model}

The MSSM is the minimal phenomenological SUSY extension of the SM. In addition to SM fermions and bosons, in MSSM two Higgs doublets with hypercharge $Y= \pm 1$ are introduced, and all the particles are assigned their superpartners. Furthermore, the most general soft-SUSY-breaking terms are added. Since all normalizable SUSY interactions conserve $B-L$ ( $B$ for baryon and $L$ for lepton quantum number), then in MSSM we have $R$-parity invariance, where $R=(-1)^{3(B-L)+2 S}$ for a particle with spin S. So, all SM particles have even $R$-parity, while the superpartners have odd $R$-parity, due to the factor $(-1)^{2 s}$. The conservation of $R$-parity implies that the lightest SUSY particle (LSP) is stable and is a final state of any SUSY particle decay chain. Another consequence is that one can produce (or annihilate) superparticles only in pairs. These two concepts have major implications on the particle collider phenomenology and cosmology. Being stable, the LSP's cosmological relic is one of the prime candidates for 
Chapter 2. Theory

dark matter. [7]. If this is the case, than LSP must be electrically neutral and not strong interacting, otherwise we would have already detected it. Then LSP should be a superpartner of neutral SM particles, $Z, \gamma$, or neutral Higgs bosons, or their linear combination (called neutralino). Since the superpartners can be produced only in pairs and decay eventually into the LSP which escapes detection, the typical signature of SUSY in colliders are events with missing energy/momentum.

The field content of MSSM prior to the EWSB is given by the gauge and matter supermultiplets. The gauge supermultiplets contain the SM gauge bosons with their fermionic superpartners: $\left(B_{\mu}, \lambda_{B}\right),\left(B_{\mu}^{i}, \lambda_{B}^{i}\right),\left(G_{\mu}^{a}, \lambda_{G}^{a}\right)$, for $\mathrm{U}(1), \mathrm{SU}(2)$ and $\mathrm{SU}(3)$ groups respectively. The matter supermultiplets are the pairs of SM matter fermions with their scalar superpartners and Higgs scalars with their fermion superpartners. the two neutral Higgs fields couple separately to the matter (s)fermions: one Higgs $\left(H_{d}\right)$ couples to charged (s)leptons and the down-type (s)quarks, while the other Higgs $\left(H_{u}\right)$ couples to up-type (s)quarks. After the Higgs fields gain the vev the particles get their masses proportional to different vev's (times the Yukawa couplings). One important parameter of the model is Higgs vev ratio $\tan \beta=\frac{v_{u}}{v_{d}}$.

The interaction Lagrangian of the MSSM is complex. The part describing the interaction of gauge bosons and gaugions with themselves and with the matter supermultiplet fields does not introduce new parameters and only depends on the corresponding gauge group coupling constant. The supersymmetric part of the Lagrangian, describing the interaction among the matter supermultiplets is described using the superpotential, which in case of $R$-parity conservation is given by:

$$
W_{M S S M}=\lambda_{u}^{i j} Q_{i} U_{j} H_{u}+\lambda_{d}^{i j} Q_{i} D_{j} H_{d}+\lambda_{e}^{i j} L_{i} E_{j} H_{d}+\mu H_{u} H_{d}
$$

The first three terms correspond to the Yukawa coupling in the SM (with the exactly the same number of parameters). The subscripts, $i$ and $j$ are generation indices. the parameter $\mu$ has mass dimension one and gives a supersymmetric mass to both fermionic 


\section{Chapter 2. Theory}

and bosonic components of the chiral supermultiplets $H_{u}$ and $H_{d}$. In mSUGRA, by requiring the EWSB the absolute value of the parameter $\mu$ can always be constrained and derived from other parameters of the theory, so we only treat $\operatorname{sign}(\mu)$ as a true parameter.

Supersymmetric particles do not have the mass of their SM partners, otherwise we would have detected them by now. We expect SUSY to be an exact symmetry that is spontaneously broken. The ultimate model should have a lagrangian density invariant under SUSY transformation, but a vacuum state that is not. This makes SUSY hidden at low energies in an analogous way EWK symmetry is hidden in the ordinary SM. An extensive treatment of SUSY breaking terms is found else where [2]. Many models of spontaneous symmetry breaking have been proposed (ref. SUSYprimer). All of these models introduce new particles and interactions at very high mass scales while is still remains unknown how this should be done correctly. To parametrize the ignorance, we can introduce extra terms which break the SUSY explicitly in the MSSM lagrangian. To maintain the hierarchy between EWK scale and Planck mass scale, these terms should be of positive mass dimension (i.e. soft). The possible soft SUSY breaking terms in the lagrangian are:

$$
\mathcal{L}_{\text {soft }}=-\frac{1}{2}\left(M_{\lambda} \lambda^{a} \lambda^{a}+\text { c.c. }\right)-\left(m^{2}\right)_{j}^{i} \phi^{j *} \phi_{i}-\left(\frac{1}{2} b^{i j} \phi_{i} \phi_{j}+\frac{1}{6} a^{i j k} \phi_{i} \phi_{j} \phi_{k}\right)
$$

SUSY breaking terms in the Lagrangian are the gaugino masses $M_{\lambda}$ and scalar mass terms $\left(m^{2}\right)_{j}^{i}$ and $b^{i j}$, plus the sclar fields' trilinear couplings $a^{i j k}$. It has been shown regourously that softly-broken SUSY theory with $\mathcal{L}_{\text {soft }}$ solves the problems of quadratic and divergences in quantum corrections to scalar masses, to all orders in perturbation theory [10]. $\mathcal{L}_{\text {soft }}$ breaks supersymmetry, since it involves only scalars and gauginos, and not their respective superpartners. The soft terms are capable of giving masses to all of the scalars and gauginos in a theory, even if the gauge bosons and fermions in chiral supermultiplets are massless. In general both (s)quarks and (s)leptons are allowed to 
Chapter 2. Theory

\begin{tabular}{||c|c|c|c|c||}
\hline \hline Names & Spin & $R$ & Mass Eigen & Gauge Eigen \\
\hline \hline Higgs bosons & 0 & +1 & $h^{0} H^{0} A^{0} H^{ \pm}$ & $H_{u}^{0} H_{d}^{0} H_{u}^{+} H_{d}^{-}$ \\
\hline squarks & 0 & -1 & $\tilde{u}_{L} \tilde{u}_{R} \tilde{d}_{L} \tilde{d}_{R}$ & $-\mathrm{ii}-$ \\
\hline & 0 & -1 & $\tilde{s}_{L} \tilde{s}_{R} \tilde{c}_{L} \tilde{c}_{R}$ & $-\mathrm{ii}-$ \\
\hline & 0 & -1 & $\tilde{t}_{1} \tilde{t}_{2} \tilde{b}_{1} \tilde{b}_{2}$ & $\tilde{t}_{L} \tilde{t}_{R} \tilde{b}_{L} \tilde{b}_{R}$ \\
\hline sleptons & 0 & -1 & $\tilde{e}_{L} \tilde{e}_{R} \tilde{\nu}_{e}$ & $-\mathrm{ii}-$ \\
\hline & 0 & -1 & $\tilde{\mu}_{L} \tilde{\mu}_{R} \tilde{\nu}_{\mu}$ & $-\mathrm{ii}-$ \\
\hline & 0 & -1 & $\tilde{\tau}_{1} \tilde{\tau}_{2} \tilde{\nu}_{\tau}$ & $\tilde{\tau}_{L} \tilde{\tau}_{R} \tilde{\nu}_{\tau}$ \\
\hline neutralinos & $1 / 2$ & -1 & $\tilde{\chi}_{1}^{0} \tilde{\chi}_{2}^{0} \tilde{\chi}_{3}^{0} \tilde{\chi}_{4}^{0}$ & $\tilde{B}^{0} \tilde{W}^{0} \tilde{H}_{u}^{0} \tilde{H}_{d}^{0}$ \\
\hline charginos & $1 / 2$ & -1 & $\tilde{\chi}_{1}^{ \pm} \tilde{\chi}_{1}^{ \pm}$ & $\tilde{W}^{ \pm} \tilde{H}_{u}^{+} \tilde{H}_{d}^{-}$ \\
\hline gluino & $1 / 2$ & -1 & $\tilde{g}$ & $-\mathrm{ii}-$ \\
\hline gravitino & $1 / 2$ & -1 & $\tilde{G}$ & $-\mathrm{ii}-$ \\
\hline
\end{tabular}

Table 2.5: Not yet discovered particles in MSSM. Gauge eigen states if denonted as "ii -" are the same as mass eigen states

mix too. After the EWSB the corresponding charge higgsinos and EWK gauginos are allowed to mix too [9]. The final particle content of the MSSM not included in SM is given in Table 2.5 .

In the postulated mass spectrum of the MSSM particles at EWK scale, the first two generation squark masses are usually degenerate, as well as the slepton masses; the third generation sfermions are usually lighter, with stop and stau being the lightest in the squark and slepton spectrum; the neutralino is LSP, the lightest Higgs ( $h$ is one of the lightest particles with mass of 115 to $200 \mathrm{GeV} / c^{2}$.

The total number of independent parameters in the MSSM is 124 (including 18 from the SM). The complete Lagrangian of MSSM is discussed elsewhere [2]. In its most general form, the MSSM is phenomenologically viable theory for the most of 
its parameter space. For a generic point there is no conservation of individual lepton numbers, flavor changing neutral current (FCNC) is not suppressed, and there are new sources of $\mathrm{CP}$ violation that are inconsistent with experimental results. As a result, the model is worth testing only in very special regions of the parameter space.

A typical phenomenological way of reducing the parameter space is to reduce the number of parameters by assuming a certain kind of universality. For example, squark flavor mixing can be constrained to that of quarks in CKM (minimal flavor violation or MFV), SUSY breaking trilinear coupling and scalar mass matrices might not depend on generations (horizontal universalities) or even more they might be diagonal in the basis where the corresponding lepton and quark mass matrices are diagonal (flavor alignment). Another way of simplifying the model is to consider model parameter at a higher energy scale (e.g. GUT) and apply constraints on the model at that scale. Inspired by GUTs, one can require the unification of gaugino mass parameters at that scale. On the other hand, one can derive SUSY breaking in the MSSM based on certain theoretical assumptions on the nature of the breaking. Some of the theoretically motivated SUSY breaking scenarios are: supergravity (SUGRA), gauge mediated SUSY breaking (GMSB), anomaly-mediated (AMSB), and SUSY GUTs. In all of the listed scenarios some type of universality at a higher energy scale is postulated. This analysis is done in the framework of mSUGRA. Its overview is given below.

In the minimal SUGRA (mSUGRA) framework, horizontal and flavor alignment is assumed. That means that all the scalar mases are degenerate and are given by a single parameter $m_{0}$; all the trilinear coupling terms are unified at some value $A_{0}$, and the gaugino masses at some value $m 1 / 2$. If we assume that there are no other SUSY breaking terms, the mSUGRA model is defined by five parameters (additional to the $\mathrm{SM}): m_{0}, m_{1 / 2}, A_{0}, \operatorname{sign}(\mu)$, and $\tan \beta$. To obtain particle spectrum and the couplings at the energy scale of the experiment at hand one uses renormalization group (RG) equations and evolves the parameters from the GUT scale. Postulating LSP and as- 
suming EWSB substantially constrain the model parameter space. Further constraints on the model are imposed by many experimental observations. Although a simplistic model, mSUGRA is useful as benchmark model. There are models that are simple extensions of mSUGRA and these usually add some type of non-universality of the parameters at the GUT scale: scalar mass non-universality (NMH model) [11] [13], or Higgs mass non-universality (NUMH model) [12] [14].

Recently, in literature, there have been many reports on serious constraints on mSUGRA models obtained by measurements, such as the anomalous muon magnetic moment $(g-2)_{\mu} / 2$ [15], determination of $b \rightarrow s \gamma[?]$ and the relic CDM density [17]. The (g-2) results call for lighter sleptons while $b \rightarrow s \gamma$ results call for the opposite, heavy squarks. Baer at al [11] reconcile the experimental findings by proposing a SUGRA model with generational mass universality relaxed. Driven by the above constraints and the constraints of FCNC which effect only the masses of the first two generations, they advocate for relatively light (few hundred $\mathrm{GeV}$ ) degenerate the first and second generation scalar masses and much heavier (multi-TeV) third generation scalar mass. Constraints from $B_{d}-\bar{B}_{d}$ allow for low values of $m_{1 / 2}$. With all the above constraints taken into account a significant portion of the parameter space still remains available.

In this NMH model, due to light slepton masses in the first two generations, chargino and neutralino decays into electron and muons are enhanced, leading to large rates for isolated multileptons plus $\mathbb{E}_{T}$ plus jets, a golden signature at collider detectors such as CDF.

\subsubsection{Trilepton Signal at the Upgraded Tevatron}

In hadron collisions, associated production of chargino and neutralino occures via quark-antiquark annihilation in the s-channel through a $W$ boson $\left(q q^{\prime} \rightarrow W^{ \pm} \rightarrow \tilde{\chi}_{1}^{ \pm} \tilde{\chi}_{2}^{0}\right)$ and in the $t$ and $u$-channels through squarq $(\tilde{q})$ exchanges, as shown in Figure 2.2. 
Chapter 2. Theory
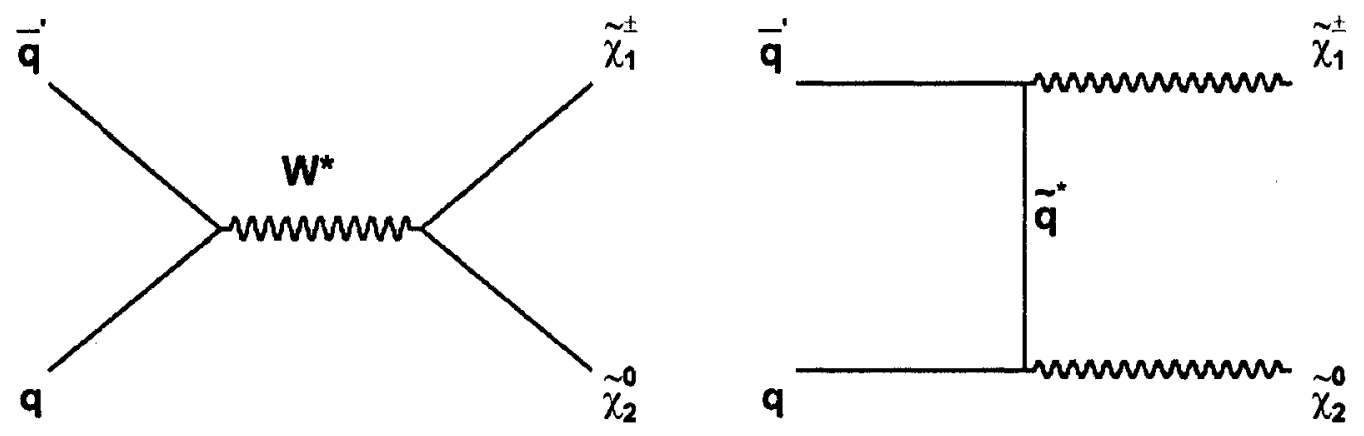

Figure 2.2: Associated $\tilde{\chi}_{1}^{0} \tilde{\chi}_{2}^{0}$ production in s-channel via virtual $W^{ \pm}$boson exchange (lef) and in $t$-channel via virtual $\tilde{q}$ squark exchange (right) at the Tevatron. For $\tilde{\chi}_{1}^{0}$ and $\tilde{\chi}_{2}^{0}$ masses near $\mathrm{W}$ and $\mathrm{Z}$ boson masses a measurable rate of production is expected at the Tevatron. Since the the above two modes interfere, the $\tilde{\chi}_{1}^{0} \tilde{\chi}_{2}^{0}$ production is enhanced for massive $\tilde{q}$.

If the squarks are light, a destructive interference between the $W$ boson and the squark exchange amplitudes supresses the cross section by as much as $40 \%$ compared to the $s$-channel contribution alone. For squarks much more massive than the SM gauge bosons, the negetive interference is supresed and the $s$-channel in $W$-resonance amplitude dominates [18].

In the mSUGRA unified model, the sleptons $(\tilde{l})$, the lighter chargino $\left(\tilde{\chi}_{1}^{ \pm}\right)$and the lighter neutralinos $\left(\tilde{\chi}_{1}^{0}, \tilde{\chi}_{2}^{0}\right)$ are typically less massive than gluinos and squarks [18]. Because of this the $3 l+\mathbb{E}_{T}$ signal from associated production and decays of $\tilde{\chi}_{1}^{ \pm} \tilde{\chi}_{2}^{0}$ is one of the most promissing channels of SUSY particles searches at Tevatron. The background to this signature from processes in the SM can be greately reduced with appropriate cuts. In mSUGRA, the weak-scale gaugino masses are realated to the universal gaugino mass parameter by $m_{\tilde{\chi}_{1}^{0}} \approx 0.4 m_{1 / 2}$ and $m_{\tilde{\chi}_{1}^{ \pm}} \approx m_{\tilde{\chi}_{2}^{0}} \approx 0.4 m_{1 / 2}$. So, a trilepton search provides valuable information on the value of $m_{1 / 2}$. We consider universal boundary condition at $M_{G U T}$ scale with a commong guagino mass $m_{1 / 2}$ and a common sfermion mass $m_{0}$. We also consider non-universal boundary conditions among sfermion masses (ref Baer). In general, smaller $m_{0}$ causes smaller sletpons 
Chapter 2. Theory

masses which enhances the branching ratio to trileptons. Feynman diagrams of threebody and two-body decays of $\tilde{\chi}_{1}^{0}$ and $\tilde{\chi}_{2}^{0}$ are shown in Figure 2.3 and 2.4. As mentioned above, the chargino and nutralino masses increase proportionaly to $m_{1 / 2}$. This increases the hardness of decaying leptons, but also reduces the cross section. This is the reason why in the search for chargino-neutralino associative production the low- $p_{T}$ channel is considered "golden channel".
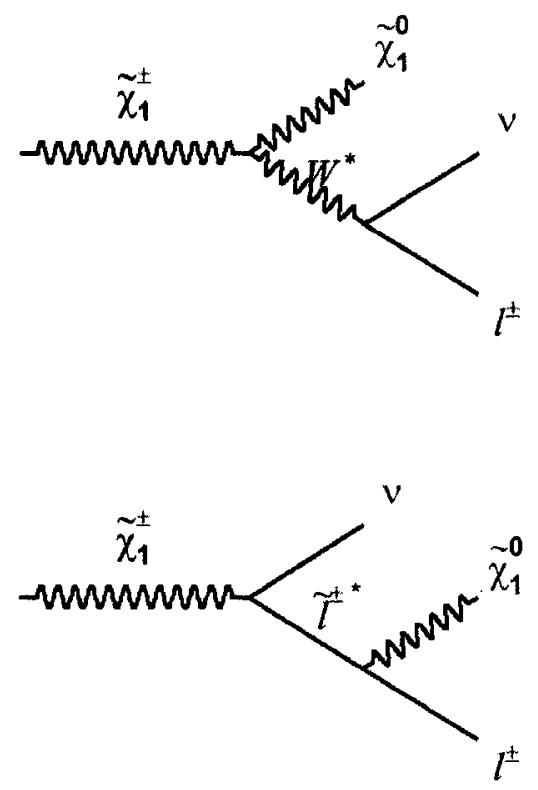
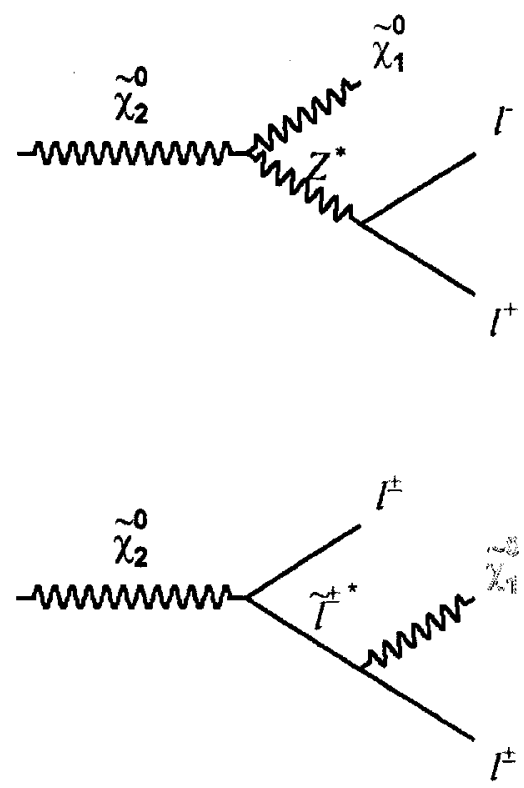

Figure 2.3: Thre-body decay modes for charginos (left) and neutralinos (right). These modes occur when the slepton masses are larger than the $\tilde{\chi}_{1}^{0}$ and $\tilde{\chi}_{2}^{0}$ masses so the decays occur via virtual $W^{ \pm}$and $Z^{0}$ bosons (top) or virtual sleptons (botom).

It is assumed that the Tevatron will deliver total of $2 \mathrm{fb}^{-1}$ of data during Run II. Than we summarize the discovery potential of the Tevatron in Run II in mSUGRA framework as:

- For $m_{0} \approx 100 \mathrm{GeV}$ and $\tan \beta \approx 2$, the trilepton signal should be detectable. 
Chapter 2. Theory
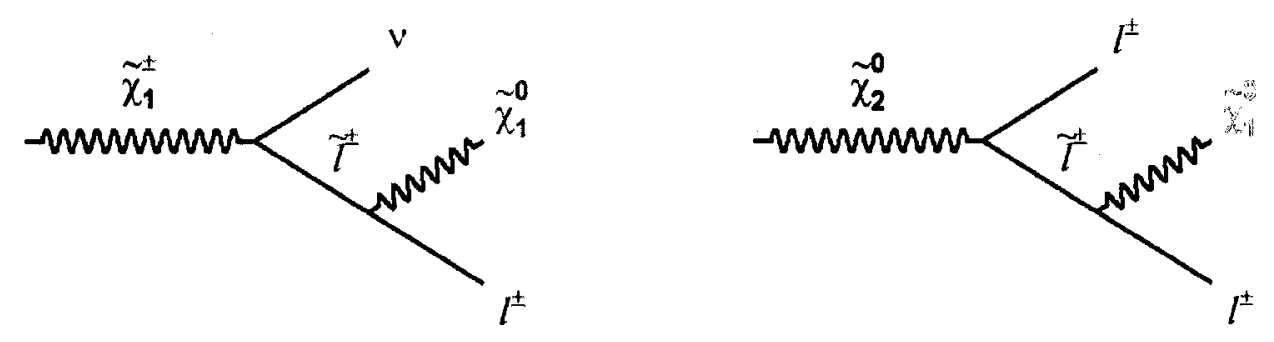

Figure 2.4: Two-body decay modes for charginos (left) and neutralinos (right). These modes occur when the slepton masses are smaller than the $\tilde{\chi}_{1}^{0}$ and $\tilde{\chi}_{2}^{0}$ masses so the decays occur via real sleptons.

However, in mSUGRA inspired model with non-universal sfermion masses, NMH model, the Tevatron's potential for discovery is best at

- $m_{0} \approx 100 \mathrm{GeV}$ and $\tan \beta \approx 10$ (ref Baer). 


\section{Chapter 3}

\section{Experimental Apparatus}

Currently, the Fermilab Tevatron is the most energetic particle accelerator on the Earth. There are two large, general-purpose detectors independently designed, constructed and installed at two interaction points on the Tevatron ring. In this analysis we use the data collected at the CDF experiment during Run II. This run started in 2001 after both detectors underwent significant upgrades. the accelerator complex was also significantly upgraded, for higher instantaneous luminosity but also for small increase in the center of mass energy $(\sqrt{s}$ from $1.8 \mathrm{TeV}$ to $1.96 \mathrm{TeV}$. An overview of the Tevatron and the detector CDF are given in this chapter.

\subsection{Fermilab Accelerator Complex}

The Fermilab accelerating complex consists of several particle accelerators shown in the drawing in Figure 3.1. The purpose of the accelerators is to produce intense proton and antiproton beams of energies as high as $980 \mathrm{GeV}$ each.

There are two common types of accelerators used at Fermilab: synchrotron and linear accelerators. A synchrotron is a ring-shaped charged particle accelerator in 


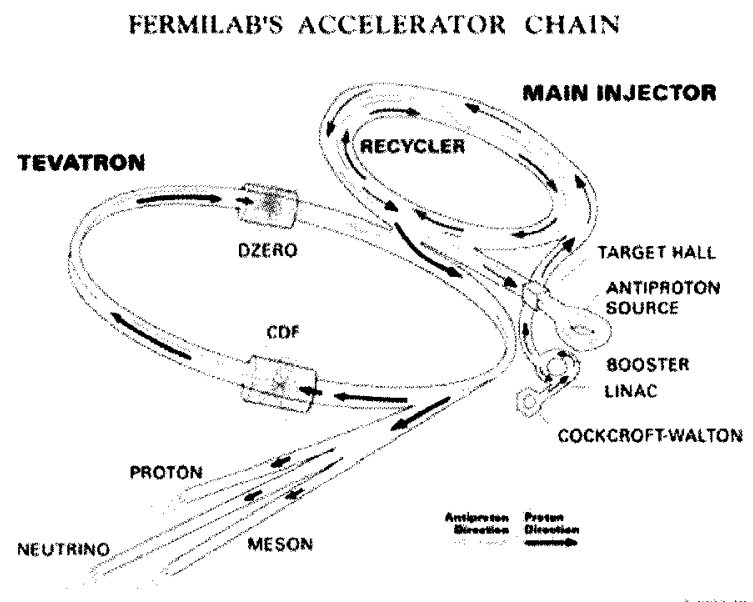

Figure 3.1: Schematic drawing of the Fermilab accelerator complex

which the magnetic field turns the particles to travel in an orbit while the electric field accelerates them. By synchronously increasing the electric and magnetic filed the charged particles gain kinetic energy while circulating thousands of times in the ring. A detailed description of operation of synchrotron can be found in literature elsewhere (ref). In linear accelerators particles gain energy in a presence of increasing electric field, traveling in a straight line.

The whole accelerator chain at Fermilab can be split into the following three stages: (1) creation and preacceleration of protons, (2) creation and preacceleration of antiprotons, and (3) injection of the particle beams for the final acceleration in the Tevatron.

Proton Source: The creation of protons begins in Cockrot-Walton chamber using gas of hydrogen molecules $H_{2}$. Negatively charged ions $H^{-}$are produced by electrical discharges in the volume of the chamber. the ions are extracted from the chamber by applying a positive voltage of $750 \mathrm{kV}$ on one side of the dome. The preaccelerated ions are fed into the linear accelerator, Linac, where the particles are accelerated in $\mathrm{RF}$ cavities to $400 \mathrm{MeV}$. The Linac is $150 \mathrm{~m}$ long and it converts a continuous stream 
of particles into discrete bunches. The $H^{-}$ions are transfered to the next accelerator called the Booster. The Booster is a synchrotron of a radius of $75 \mathrm{~m}$. The Linac injection pulse length is about 10 times longer than the time particles need to go once around in the Booster. Once in the Booster, the $\mathrm{H}^{-}$ions pass through a thin carbon film which strips them off of electrons leaving the bare protons. The proton bunches in the Booster pass through a sequence of RF cavities arranged in a ring where they are accelerated by oscillating electric field. After several thousand cycles, the protons reach energy of $8 \mathrm{GeV}$ and the bunches become dense constantly adding protons from $\mathrm{H}^{-}$ ions coming from the Booster. At the next stage, the proton bunches are transfered into the Main Injector, yet another but larger synchrotron. The Main Injector has radius of seven times that of the Booster, which enables the protons to acquire energies of 150 $\mathrm{GeV}$. Once at that energy and with around $5 \cdot 10^{1} 2$ per bunch, the protons are ready for the final acceleration in the Tevatron, the final synchrotron in the chain.

Antiproton Source: Two small synchrotrons, the Debuncher and the Accumulator, are referred to as as the antiprotonssource. Protons with energy of $120 \mathrm{GeV}$ are extracted from the Main Injector and transfered to the target area where they are used to bombard a nickel target. As the result of proton scattering on the target, a spray of various particles is produced at many angles. These particles are focused with the Lithium lens and then the antiprotons are extracted by a magnetic charge-mass spectrometer. These antiprotons are moved to the Debuncher, where the spread in the particle momenta is reduced by a stocastic cooling process, and further sent to the Accumulator where they are stored till enough have been accumulated. The stack rate for antiprotons is about $4 \cdot 10^{10}$ protons per hour. Stacking continues till $10^{12}$ protons are accumulated. The antiprotons are then sent back to the Main Injector where they are accelerated to $150 \mathrm{GeV}$. Since protons and antiprotons are of opposite electric charge, they are accelerated in opposite directions without colliding in the Main Injector. 


\section{Chapter 3. Experimental Apparatus}

The Main Injector sends the proton and antiproton bunched beams into the Main Ring, the Tevatron. It was specifically built for this purpose. Before its existence, Main Ring accelerator could only accelerate particles to $400 \mathrm{GeV}$. After accepting 150 $\mathrm{GeV}$ particles from the Main Injector the Main Ring is now capable of accelerating them to $980 \mathrm{GeV}$ each, almost $1 \mathrm{TeV}$, therefore the name Tevatron.

The Tevatron's ring is $1 \mathrm{~km}$ in radius. Very powerful magnetic fields $(4.2 \mathrm{~T})$ are needed along its ring to maintain the motion of the energetic particles in the orbit. This is obtained by using superconducting magnets made of Niobium-Titanium alloy that are cryogenically cooled to about $4.6 \mathrm{~K}$ with liquid Helium. Thirty six bunches each of protons and antiproton circulate in opposite directions int the Tevatron. There are two point along the ring designated as B0 and D0 (see Figure), where the beams are focused and brought to collision. the result of such collisions are observed by two multi-purpose detectors installed at the these points. The collider Detector at Fermilab (CDF) and The D0-Detector.

The process of loading protons and antiprotons int the Tevatron is called the shot setup, and it begins when the size of the antiprotons stack in the Accumulator is large enough. The proton bunches are injected first by the transfer system with a fast kicker magnet having 396 ns rise time. The 36 bunches are injected in 3 trains of 12 coalesced bunches with a abort gap between each train of $2.617 \mu \mathrm{s}$. In fact, a single proton bunch in the Tevatron is a product of merging of several bunches prepared int eh Booster providing an increased intensity of about $30 \cdot 10^{10}$ protons per bunch. The antiproton bunches are injected similarly to the protons when either of the three abort gaps in the proton beam passes the antiproton kicker magnet. Like with protons, each bunch is a marge of several antiproton bunches prepared in the Accumalator resulting in about $30 \cdot 10^{10}$ antiparticles per bunch.

Once the Tevatron loading is complete, beams are accelerated to the maximum energy and a stable configuration is reached the data taking period (store) begins. 
During this period the luminosity decrease due to loss of particles in $p \bar{p}$ collisions and beam-gas interactions. Also, the beams start to heat up and their effective width increases due to long range beam-beam interactions. The instantaneous luminosity is given by the formula

$$
R=\sigma \mathcal{L}=\sigma \frac{\left\{\mathcal{N}_{\mathrm{L}} \mathcal{N}_{\sqrt{ } \mathcal{N}_{\precsim}}\right.}{\epsilon \diamond \sqrt{\int_{\sqrt{ }}^{\epsilon}+\int_{\mathfrak{\checkmark}}^{\epsilon}}}
$$

where $\sigma$ is effective cross section of a particular interaction, $f$ is the bunches's revolution frequency, $N_{b}$ is the number of bunches in the beam, $N_{p}$ and $N_{\bar{p}}$ are the number of protons and antiprotons respectively, $s_{p}$ and $s_{\bar{p}}$ are the transverse sizes of the proton and antiproton beams at the interaction point respectively. From the above expression one can see that luminosity increases as number of protons and antiprotons increases or as effective beam sizes decrease. If the cross section of specific interaction is $\sigma$ then the interaction rat $\mathrm{R}$ is proportion to the instantaneous luminosity L. While detectors collect data, a new stacking period begins in the Accumulator preparing antiprotons fort the next store. A typical store lasts for 15 hours. After that time, it is more efficient to abort the data tacking in detectors an start a new cycle with fresh beams. The time between two stores is typically 2 hours and it can be used for detector calibration.

\subsection{Collider Detector at Fermilab}

\subsubsection{Overview}

CDF is a multipurpose magnetic detector of high energy $p \bar{p}$ collisions. A general view of CDF is shown in Figure 3.2 and a cross section view is shown in Figure 3.3. 


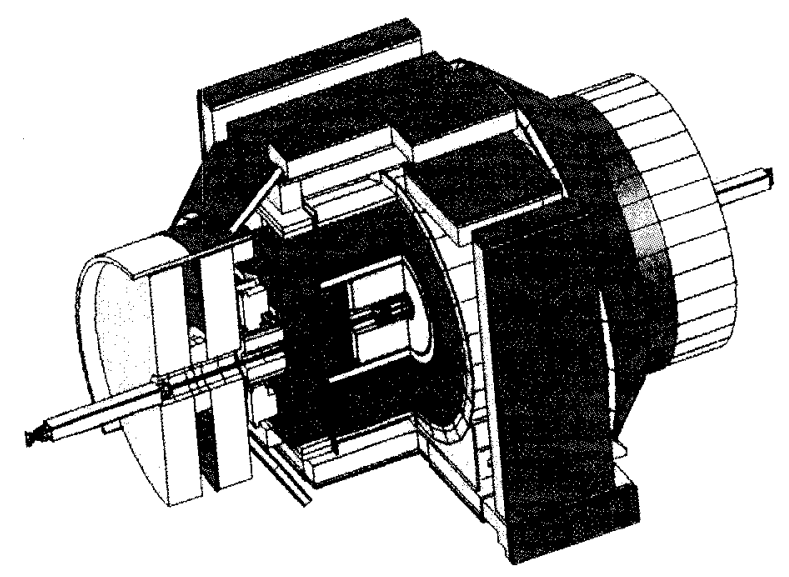

Figure 3.2: Isometric view of the CDF with cut-away quarter

The detector was significantly upgraded for Run II (ref UpgradeManual) to be compatible with the increased luminosity of the Tevatron and shorted bunch crossing time leading to higher $p \bar{p}$ collision rate. For improved momentum resolution, b-tagging, electron and muon identification in Run II some parts of the detectors were replaced and new hardware was added to the CDF.

The CDF consists of the following subdetectors:

- The tracking system is the innermost part of CDF used for particle charge and momentum measurements. This system is placed inside a supeconducting solenoid of $4.8 \mathrm{~m}$ long and $1.5 \mathrm{~m}$ radius, which produces a $1.4 \mathrm{~T}$ magnetic field coaxial with the beam direction.

- The calorimetr system is composed of electromagnetic (EM) and hadronic (HAD) scintillator-based sampling calorimeters surrounding the solenoid in a projective 
Chapter 3. Experimental Apparatus

tower geometry. The EM calorimeter features an embedded proportional chamber at shower maximum for precise electron identification and reconstruction.

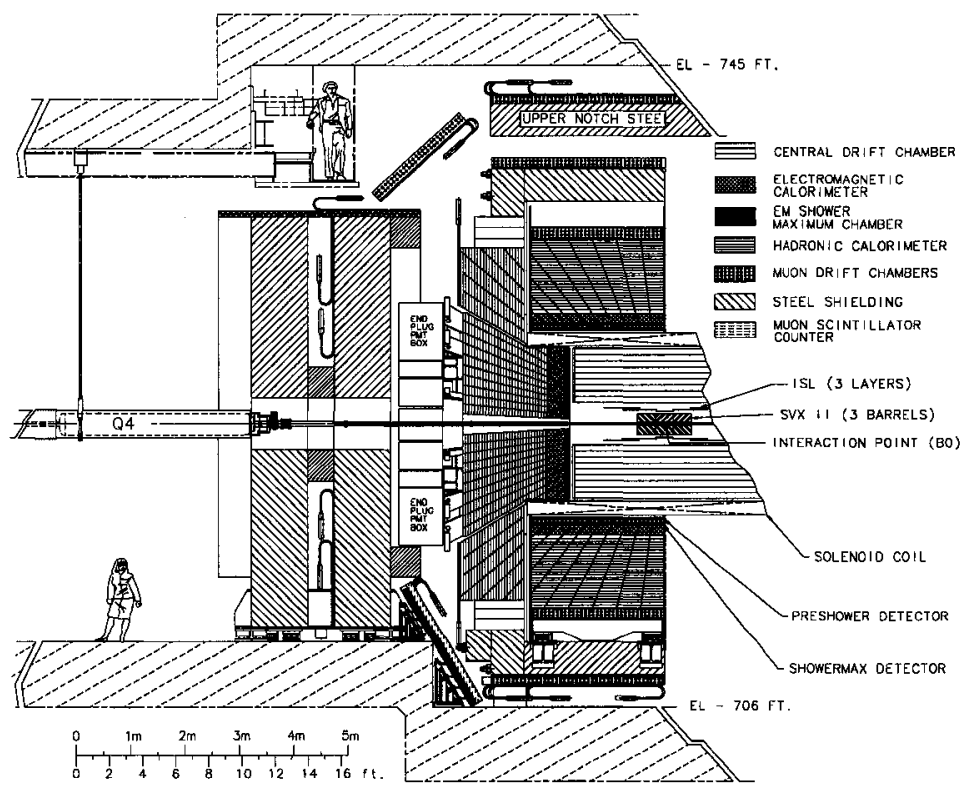

Figure 3.3: Elevation view of one half of the CDF 


\section{Chapter 3. Experimental Apparatus}

- The muon system, made of drift wire chambers, is located on the exterior of $\mathrm{CDF}$. Only high energy muons are able to pass slabs of steel beyond which the muon detectors are located.

A Cartesian coordinate system is chose with the origin at the geometrical center of the detector. The $x$ axis is defined in the direction radially outward from the ring's center, the $y$ axis is pointing directly upward, and the $z$ axis is in the antiprotons' beam directions of the cylindrical shape of the detector it is more convenient to identify particle directions and other location within the detector in a cylindrical-like coordinate system. The three coordinates $(z, \phi, \eta)$ where $\phi$ is the azimuthal angle in $x-y$ plane and $\eta$ is the pseudorapidity expressed in terms of the polar angle $\theta$ as

$$
\eta=-\ln \left(\tan \frac{\theta}{2}\right) .
$$

The pseudorapidity is chosen as a coordinate because of its close relationship with the rapidity known from special relativity, $y$,

$$
y=-\frac{1}{2} \ln \left(\tan \frac{E-p_{z}}{E+p_{z}}\right),
$$

where $E$ is energy and $p_{z}$ is the $z$ component of the momentum of the particle. Rapidity is a convenient variable to use in high energy systems due to its additive nature under Lorentz transformation. In a boosted frame of reference moving with velocity $\beta$ with respect to some initial frame of reference, rapidity transforms as

$$
y \rightarrow y-\tanh ^{-1} \beta
$$

For highly relativistic particles $\eta$ is a good approximation to $y$. Because of $\Delta \eta$ invariance under Lorentz transformation the distribution $\frac{\delta N}{\delta \eta}$ also remains invariant. 
Chapter 3. Experimental Apparatus

This is the reason why the CDF calorimeter is segmented in towers of equal units of pseudorapidity. If an opening angle between two particles if defined as

$$
\Delta R=\sqrt{(\Delta \eta)^{2}+(\Delta \phi)^{2}}
$$

then it is Lorentz invariant as well since both $\Delta \eta$ and $\Delta \phi$ are.

\subsubsection{Tracking System}

The Tracking System is the inner most part of the CDF. Its purpose is to track the path of particles which are products of $p \bar{p}$ collisions. When a charged particle traversed the medium of the tracking system, it produces ionization that is collected on a localized electrode and which is called a hit. A sequence of hits spatially distributed are later used to reconstruct the path the product particle traversed. This whole precess is called tracking. The Tracking System consists of two main components: Silicon Detector at very small radii and Central Outer Tracker cylindrically surrounding the former. Both detectors are submerged in into a uniform magnetic filed $B=1.4$ Tesla whose direction is along $z$ axis.

The trajectories of charged particles inside of a magnetic field are helices. A helix can be described by the following parameters:

- $z_{0}-z$ coordinate of the point of the closest approach to the $\mathrm{z}$ axis

- $d_{0}=q\left(\sqrt{x_{0}^{2}+y_{0}^{2}}-R\right.$ - distance from the pint of closes approach to the $z$ axis, impact parameter. $q$ is the charge of the particle and $\left(x_{c}, y_{c}\right)$ is the projection of the center of helix onto $x-y$ plane.

- $\phi_{0}$ - azimuthal angle of the tangent to the trajectory at the point of closest approach to $z$ axis 
- $\cot \theta$ - ratio of helix step to its diameter, helix pitch

- $C=\frac{q}{2 R}$ - helix curvature

The above parameters can be used to calculated the particle's transverse and longitudinal momenta:

$$
p_{T}=\frac{q B}{2|C|}, p_{z}=p_{T} \cot \theta
$$

The track parameters are determined by the helical track fit. The input to this fitter is a set of spacial coordinates of the measured hist which specially preselected with the pattern recognition algorithm. The primary vertex of event is determined from the reconstructed track in the event by fitting them to the same space point and using the beam line position as a constrain. Tracks with hits in the silicon detector are reconstructed with high precision and can be used to reconstruct secondary verticies. The schematic side view of the one quarter of tracking system is shown in Figure 3.4. The parameters of each component of tracking system will be briefly described below.

\section{Silicon Detector}

the silicon detector consists of eight concentric cylindrical layers of silicon strips surrounding the beam pipe. The layers span radii from $1.35 \mathrm{~cm}$ to $28 \mathrm{~cm}$ and extend from $90 \mathrm{~cm}$ to almost $2 \mathrm{~m}$ along the pipe line. The detector is divided into three cylindrical sub-systems: Layer-00, SVXII, and ISL, listed in the increasing distance from the pipe.

The Silicon Vertex Detector (SVXII) is the most important component of the silicon tracking system. It consists of 5 layers of double-sided microstrip detectors. The most inner and outer layers have the radii of $2.5 \mathrm{~cm}$ and $10.7 \mathrm{~cm}$ respectively. Along the beam pipeline the SVXII covers 2.5 -sgima of the $p \bar{p}$ luminous region, corresponding 


\section{CDF Tracking Volume}

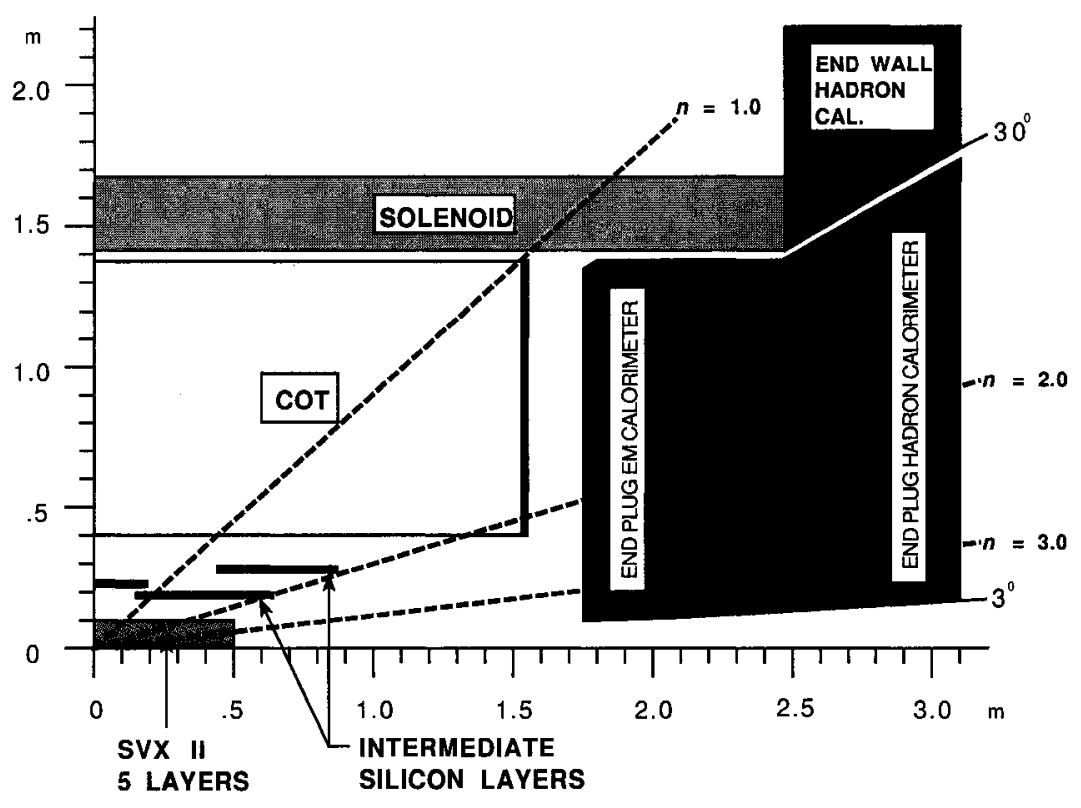

Figure 3.4: Schematic drawing of one quarter of CDF tracking system. Parts of calorimetry system (Plug and End Wall) are also shown on the drawing

to the length of $96 \mathrm{~cm}$, and in a pseudorapidity it covers $\eta<2.5$ as indicated in Figure 3.4. In the axial direction, SVXII is divided into three barrels each $29 \mathrm{~cm}$ long with 
beryllium bulkhead at each end. The bulkhead support the five silicon layers and the cooling system for readout electronics. In the plane transverse to the beam line, each layer is segmented in 12 azimuthal wedges as shown in Figure 3.5.

Figure 3.5: CDF Silicon Vertex Detector - SVXII

To minimize residual interaction, the silicon sensors are supported by very light substrates forming a basic structural unit called barrel. SVXII contains three such barrels. One side of silicon sensors is made of finely spaced $p$-type silicon strips runig in axial direction allowing measurements in $r-\phi$ plane. On the other side of silicon sensors, $n$-type silicon strips are rotated forming either $90^{\circ}$ or $\pm 1.2^{\circ}$ stereo angels allowing mesurements of $r-z$ plane. When a signal is detected on a small cluster of axial and stereo strips and the hit position 3-space is determined. The error on the individual hit position is abut $12 \mu \mathrm{m}$.

The readout units are mounted directly on the silicon surface on both ends of each ladder. This minimizes electronic noise. Each wedge has 44 readout chip sets and each chip has 128 channels with charge sensitive amplifiers. The total number of readout channels for six barrel ends is 405,504. The signals are converted by special port card from electrical to optical form, and transmitted to external electronics at $53 \mathrm{MHz}$ rate. The highly parallel readout permits the entire detector to be read in approximately 10 $\mu \mathrm{s}$.

To further improve impact parameter resolution, Layer-00 is positioned directly on the beam pipe at a radius of $1.6 \mathrm{~cm}$. The length of Layer-00 is $80 \mathrm{~cm}$ and its azimuthal structure is similar to the the SVXII. Layer-00 uses radiation tolerant single sided microstrip sensors. The narrow sensors laying closer to the pipe, have 128 channels and wider sensors have 256 channels with a strip width of $50 \mu \mathrm{m}$. (see Figure 3.6. 
Chapter 3. Experimental Apparatus

Figure 3.6: Layer00 viewed in the plane transverse to the beam. Layer00 sensors are colored in red. The two most inner layers of SVXII surrounding Layer00 are shown as well.

The ISL is placed outside SVXII as depicted in the cartoon of Figure 3.4. There is one cylindrical layer installed in the cenetral region $|\eta|<1.0$ at radius of $22 \mathrm{~cm}$. Two two other layers are installed at radii of $20 \mathrm{~cm}$ and $29 \mathrm{~cm}$, covering the pseudorapidity region to $1.0<|\eta|<2.0$. Double-sided silicon sensors are used in ISL ladders. On axial side, the silicon mcirostrips are spaced by $55 \mu \mathrm{m}$ and on the stereo side by $73 \mu \mathrm{m}$ with $1.2^{\circ}$ stereo angle. To optimize the readout time, only every other strip is readout in ISL, amounting to total of 268,000 readout channels. The signal hit resolution in ISL is 16 and $23 \mu \mathrm{m}$ on axial and stereo layers respectively. This is worse than the hit resolution in SVXII. The purpose of ISL subdetector is to provide extra hits between SVXII and the Central Outer Tracker (COT) which we describe in the following subsection.

Overall, the silicon system measures the impact parameter (which is its main purpose) of high $p_{T}$ tracks with a resolution of about $40 \mu \mathrm{m}$, including 25-30 $\mu \mathrm{m}$ contribution from the beam width. The long term performance of the silicon system depends on the radiation damage it acquires over the time of data taking. Its most vulnerable components, the innermost layer of SVXII and Layer00, are expected to last at least till $7.4 \mathrm{fb}^{-1}$ of data are taken.

\section{Central Outer Tracker}

The Central Outer Tracker (COT) is a cylindrical, multi-wire open-cell drift chamber, designed to find tracks of charged particles in the central region $\eta<1.0$. As shown in Figure 3.4, the COT is located outside the silicon detector and inside the solenoid. This means that COT as well is emerged in the magnetic field of $1.4 \mathrm{~T}$. The COT covers 
Chapter 3. Experimental Apparatus

\begin{tabular}{llll}
\hline Superlayer & Radius at center $(\mathrm{cm})$ & Number of cells & Stereo Angel \\
\hline
\end{tabular}

Table 3.1: COT layers summary

the volume between $\mid z ; 155 \mathrm{~cm}$ and $44 \mathrm{~cm}<r<155 \mathrm{~cm}$. It is hermetically closed and filled with a 50:50 mixture of Argonne-Ethane gas, which has a drift velocity of 50 $\mu \mathrm{m} / \mathrm{ns}$. Small drift times are important for preventing the subsequent event's signal pile-up. This becomes essential at high collision rates for which Tevatron is designed.

A charged particles pass through the COT and leave a trail of ionized gas behind it. The electrons drift to sense wires strung between the end plates of COT. The sense wires are placed in elctrostatic drift field created by filed-shaping wires and cathode field panels, both running parallel to the sense wires. the COT cell consist of 12 wires alternating with 13 potential wires as shown in Figure 3.7. Two adjacent cell share the same field panel, and four shaper wires (two at each side) serve to close the cell electrostatically. Because of the magnetic field in the $z$ direction, the ionization electrons do not move along the electric field lines but rather cross them at the angle of $35^{\circ}$ in the case of COT. Therefore, the COT cells are rotated by that same angle in respect to the radial direction to reduce the drift length.

The field panels are kept at ground potential ans sense wires are operated under potentials of approximately 2 and $3 \mathrm{kV}$ respectively. Within a cell the actual voltages are slightly varied from wire to wire to maintain a uniform drift field.

There are total of of 30,240 sense wires in COT. They are arranged in eight radially spaced superlayers as shown in Figure 3.8 .

There are 12 sense wires in one cell, so there is 96 possible measurements per track traversing the COT radially. The even layers are axial, i.e. wires are in the planes parallel to the beam, and odd layers have a small stereo angle of $\pm 3^{\circ}$. The number of cells and their radii are summarized in Table 3.1 
Chapter 3. Experimental Apparatus

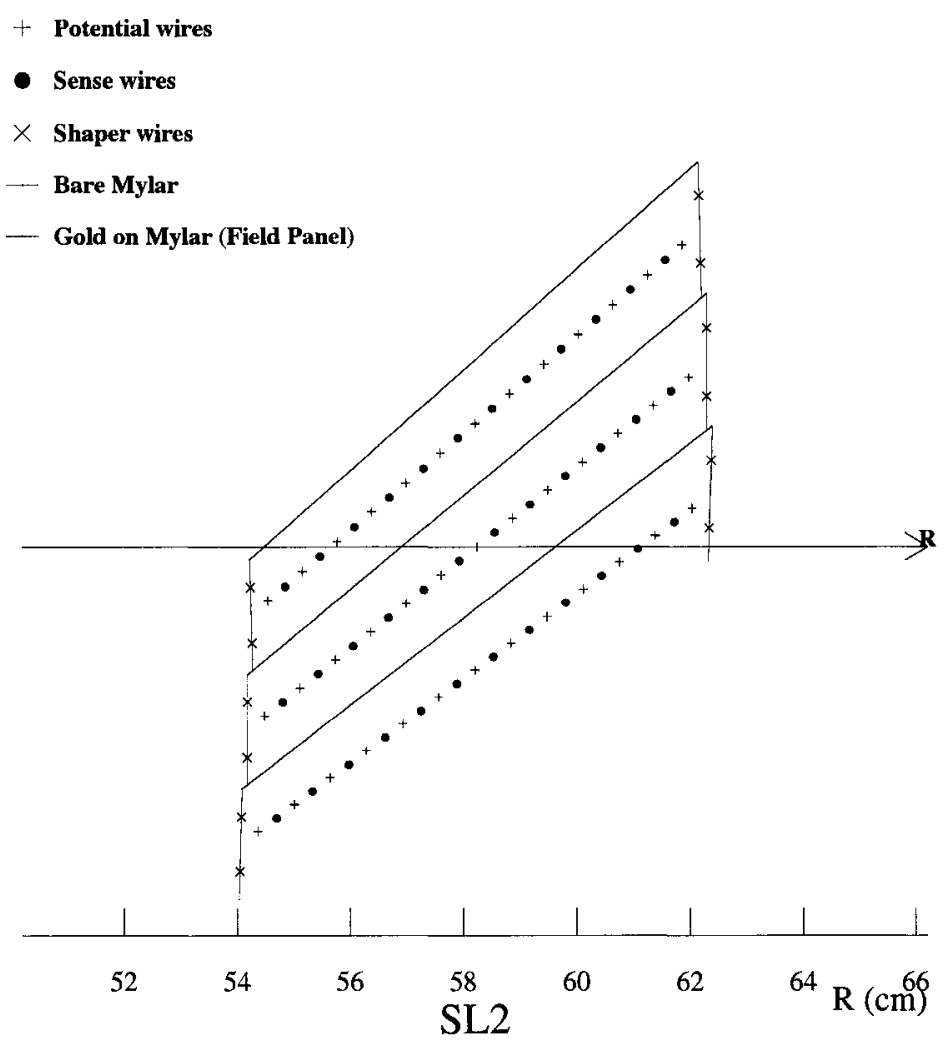

Figure 3.7: Transverse plane view of three COT cells. The $R$-arrow shows the radial direction. Electrostatic field is perpendicular to the field panels and drift velocity is perpendicular to the radius

The signals from sense wires are readout by electronics which are directly placed on the chamber end caps. Specially designed pattern recognition logic in the Level-1 
Chapter 3. Experimental Apparatus

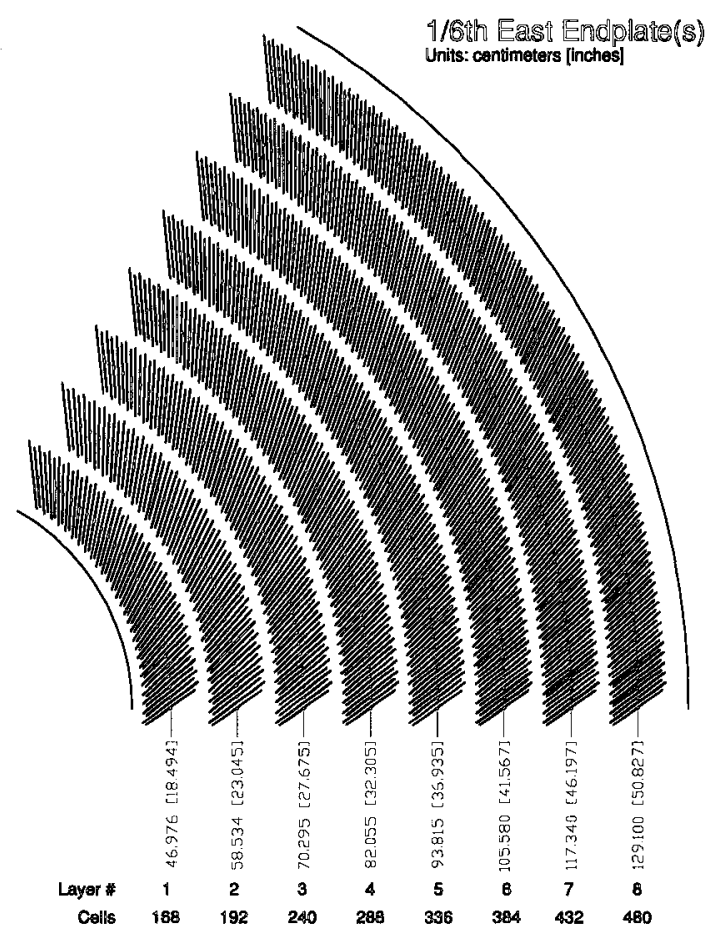

Figure 3.8: COT end plates. The wire-plane slots are grouped into eight concentric super layers. The slots are tilted by $35^{\circ}$ with respect to radial direction

trigger is used to recognize multiple hits from single wire. The resolution of a single hit is measured to be about $140 \mu \mathrm{m}$, which corresponds to the transverse momentum 
resolution of $\delta p_{T} / p_{T}^{2} \approx 0.15 \%(\mathrm{GeV} / c)^{-1}$. In absolute magnitude the tracks can be measured down to $300 \mathrm{MeV} / c$. If both the Silicon Detector and the COT are used, the transverse momentum can be measured with resolution even lower than $\delta p_{T} / p_{T}^{2} \approx$ $0.10 \%(\mathrm{GeV} / c)^{-1}$.

\subsubsection{Calorimetry}

The purpose of the CDF calorimeter system is to measure the energies of the charged and neutral particles produced in $p \bar{p}$ collisions. The absorption and scintillation materials are the main components of each calorimeter. As particle traverses the calorimeter it looses its energy by interacting with the dense absorption material and produces a shower of secondary particles (ref W.R. Leo). The number of secondary particles is proportional to the energy of the incident particle. A scintillator material is used to collect these secondary particles and release the equivalent amount of energy via light, i.e. photons. The light from scintillators is read out by light guides and converted into electrical signals by photomultiplier tubes (PMT) and amplifiers. The strength and position of the signal are the information used in the event reconstruction. The information from calorimetry combined with the tracking information is the essence of particle type identification.

The resolution of energy measurement in a calorimeter, $\frac{\sigma E}{E}$, is dominated by statistical sampling fluctuations and the photo-statistics of the PMTs, which is inversely proportional to the square root of the incident energy. Calibration errors, non-uniform response of the calorimeter, and electronic noise also contribute to the resolution.

The calorimeter detectors are installed just outside the solenoid providing $2 \Pi$ azimuthal coverage out to $|\eta|<3.6$ 3.4. The Central and the Plug Calorimeters cover regions of $|\eta|<1.1$ and $1.1<|\eta|<3.6$, respectively. The gap between the two is covered by the End Wall Calorimeter. Both Central and Plug calorimeters consist of two 
sections: Electromagnetic (EM) and Hadronic (HAD), while the End Wall has only hadronic system. Radially, EM calorimeter is placed in front of the HAD calorimeter toward the collision point. Such positioning is motivated by difference in penetration lengths for electrons/photons and hadrons. The high energy electrons loose their energy in the calorimeter via bremsstrahlung or photon radiation. Photons primary means of loosing energy is by their conversion into electron-positron pairs. In both cases electromagnetic showers are produced. The interactions of hadrons is more complicated since they couple weekly and strongly as well. At CDF's calorimeter hadrons deposit energy mostly in inelastic collisions with the nuclei of the absorbing material. Nuclear interaction cross section is smaller than that of electromagnetic, therefore hadrons travel longer distances then electrons before loosing their energies. For electrons and photons we define radiationlength $X_{0}$ as the distance over which the particle looses $1-e^{-1}$ of its energy. The nuclear interactionlength $\lambda_{I}$ is defined as a mean free path of hadron before it undergoes any inelastic scattering.

The central calorimeter consists 24 azimuthal wedges as shown in Figure ??. Each wedge covers $15 o$ in azimuthal direction and $250 \mathrm{~cm}$ in $z$, both positive and negative direction. In radial direction, central electromagnetic calorimeter (CEM) covers 173 $<r<208 \mathrm{~cm}$, which corresponds to 18 radiation lengths. Radially outward, central hadronic calorimeters (CHA) is placed. Both CEM and CHA are segmented in towers of equal pseudorapidity $\Delta \eta=0.11$. In CEM, the $0.5 \mathrm{~cm}$ thick plastic scintillators are interspersed with $0.32 \mathrm{~cm}$ thick layers of lead that serves as absorber. Each scintillator is read out by two PMT. The resolution on energy measurement is $\frac{\sigma E}{E}=13.5 \% / \sqrt{E_{T}} \oplus$ $2 \%$, with the constant term added in quadrature. In CHA, scintillators are twice as thick, $1 \mathrm{~cm}$, and the absorption layers made of steel are $2.5 \mathrm{~cm}$ thick. The wall hadronic calorimeter (WHA) has a structure similar to that of CHA, except that its absorption steel layers are $5 \mathrm{~cm}$ thick. This is to accommodate for very energetic hadrons at this $\eta$ range. The width of CHA and WHA in terms of $\lambda_{I}$ and their resolutions are listed in Table ??. 


\section{Chapter 3. Experimental Apparatus}

The plug calorimeter as well has the electromagnetic (PEM) and hadronic (PHA) parts to it, each with 23 scintillator-absorber layers. The segmentation of PEM and PHA into $\eta$ towers is finer than than in the case of CEM and CHA. This optimizes electron identification in $b$-jets. In PEM, absorber layer is $5 \mathrm{~cm}$ thick calcium-tin-lead alloy enclosed between steel plates. In CHA, absorber layer is $5 \mathrm{~cm}$ thick iron. Energy resolutions and the widths of PEM and PHA in terms of $\lambda_{I}$ are listed in Table ?? More specification details can be found in (ref IDR).

\subsubsection{Muon System}

The energetic muons produced in $p \bar{p}$ collisions manage to escape the magnetic filed of the solenoid. Muons do not interact heavily with the calorimeter. At such high energies muons Coulomb-scatter in the calorimeter without developing a shower. The detection of muons is performed with proportional drift chambers and scintillators, both placed at the most outer part of the detector. Their muon system is composed of four subdetectors covering the following regions in pseudorapidity: Central Muon (CMU) and Central Muon Upgrade (CMUP) chambers cover the region of $|\eta|<0.6$, the Central Muon Extension detector covers the region of $0.6<|\eta|<1.0$, and the Intermediate Muon (IMU) detector extends the coverage further to about $|\eta|=2.0$. The CMU and CMP cover $84 \%$ and $63 \%$ of the solid angle respectively. Overlap region of CMU and CMP is about $53 \%$ of the solid angle. The complete coverage in $\eta-\phi$ space of muon detectors is shown in 3.9 .

The CMU detectors are located within the central calorimeter wedges, directly behind the CHA at the radius of $347 \mathrm{~cm}$ (see Figures 3.2 and 3.3). The material in the yoke of the magnet and in the calorimeter serve as an absorber for hadrons shielding the muon chambers. The amount of this shielding for CMU is equivalent to 5.5 hadron interaction lengths. This sets the muon sensitivity threshold to $1.4 \mathrm{GeV} / c$. Like the 
Chapter 3. Experimental Apparatus

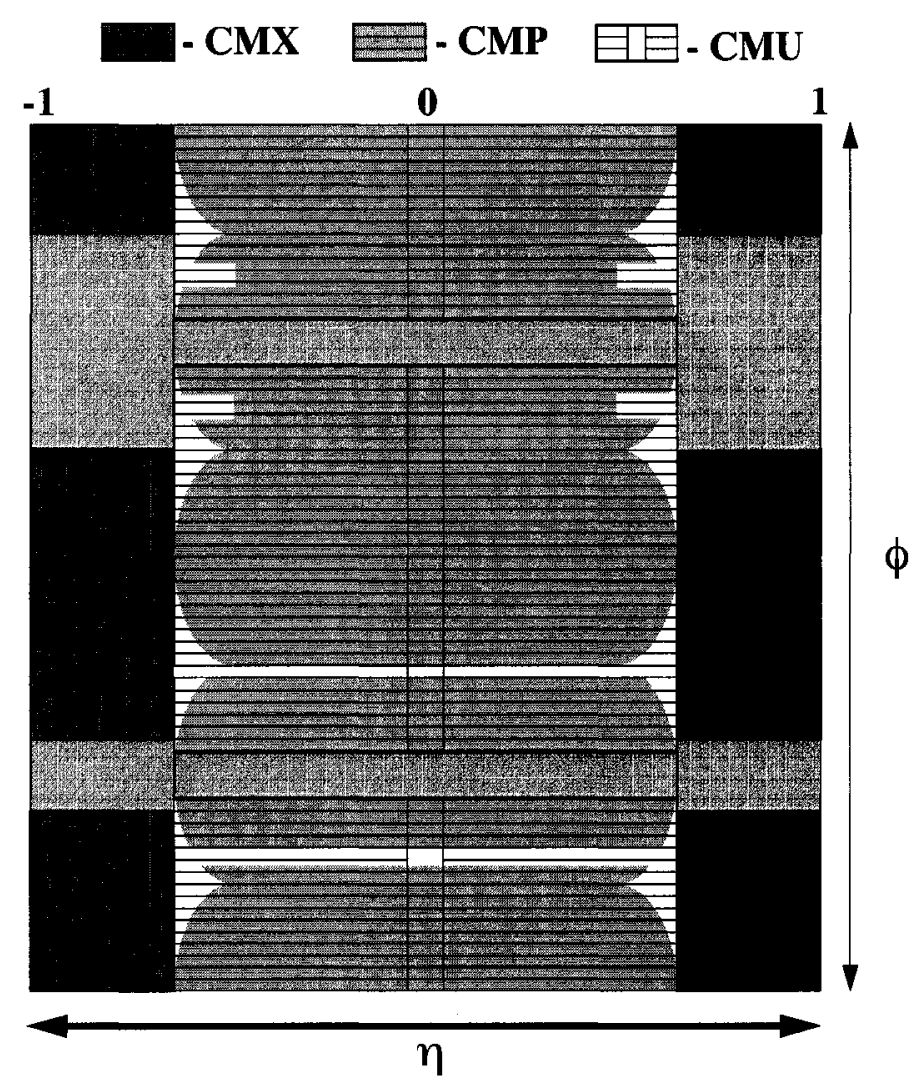

Figure 3.9: Coverage of muon systems in $\eta-\phi$ space

calorimeter, the CMU is split in 24 azimuthal wedges, $15^{\circ}$ each. Instrumented part is of the wedge is only $12.6^{\circ}$ leaving $2.4^{\circ}$ gaps between the chambers. Each wedge is further segmented in $\phi$ into three towers. Each tower is made of four radially stacked 
rectangular drift cells. There is a sense wire at the center running along a drift cell in the $z$ direction, connecting the end plates of the cell. The length of the cell in the $z$ direction is $226 \mathrm{~cm}$. The first and the third cell in a stack are offset by $2 \mathrm{~mm}$ in $\phi$ direction with respect to the second and fourth cell. This is done to avoid ambiguous $\phi$ measurement. Single hit position can be measured with resolution of $250 \mu \mathrm{m}$ in the $r-\phi$ plane and $1.2 \mathrm{~mm}$ in the $r-z$ plane. The $z$ coordinate is determined by comparing the pulse heights at each end of the sense wires. It the hist are observed on at least 3 of the 4 cells in one stack, or 3 or 4 cells from distinctive layers in two neighboring stacks, a muon stub is reconstructed.

The CMP chambers for a rectangular "box" around the central part of CDF. To further reduce the number of hadrons entering the CMP detector, an additional layer of $60 \mathrm{~cm}$ of steel is placed in front of the CMP detector. This provides extra $2.3 \lambda_{I}$ and raises the muon momentum threshold to $2.2 \mathrm{GeV} / c$. The drift cells and their arrangement in CMP are similar to those in CMU. The outer surface of the CMP is lined with scintillator counters (CSP) used to obtain timing information. Because of the rectangular shape of the CMP, its $\eta$ coverage varies as a function of $\phi$. However, at some values of $\phi$, CMP covers CMU gaps.

The muon coverage is extended by additional muon chambers organized in the CMX and IMU detectors. The CMX is made of two conical arches of drift tubes with layers of scintillators (CSX) installed a each end of the central part of the detector. No additional absorption material is added before CMX, since at these polar angles $\left(45^{\circ}-55^{\circ}\right)$ there is enough detector material to stop potential hadrons from reaching it.

The IMU consists of a barrel of drift chambers and scintillator counters placed on both sides of toroid magnets. The IMU drift chambers, scintillators, and read-out electronics are identical to the ones used in CMU. Data used in this analysis have no information from the CMU, since its trigger was not implemented. We discuss triggers in one of the sections below. 


\subsubsection{Shower Detector}

The electromagnetic showers are very narrow in transverse direction. The Moliere radius, within which approximately $90 \%$ of the total energy of a EM shower is deposited, has typical vaolues of $\approx 2 \mathrm{~cm}$ int the CEM. To enable precise measurement of the location of EM shower, finely segmented detectors are embedded in central (CES) and plug (PES) electromagnetic calorimeters. These detectors are located at about six radiation lengths from the front face of the CEM and PEM detectors, which is were the average shower maximum is expected to occur.

The CES detector consists of proportional strip and wire chambers that provide position and transverse shape of the electromagnetic showers in both $z$ and $r-\phi$ directions. The position resolution of CES measurement is $0.2 \mathrm{~cm}$ for electrons with energy of $50 \mathrm{GeV}$. The PES detector is made with of $0.5 \mathrm{~cm}$ pitch scintillator strips and arranged in two layers. The direction of strips in these layers are rotated at $+22.5^{\circ}$ and $-22.5^{\circ}$ with respect to the radial direction, to provide a tow dimensional position measurement. The PES position resolution is about $0.1 \mathrm{~cm}$.

There is also a set of multiwire proportional chambers in from of the CEM called Central Preshower (CPR) chambers. These chambers detect elctromagnetic showers that begin in the solenoid magnet material, providing enhanced photon and soft electron identification. There is no dedicated preshower detector in front of PEM, instead the first scintillator layer of PEM is made thicker $(1 \mathrm{~cm})$ to yield more light and is read out separately. This layer is called PPR and it is used to statistically separate prompt $\gamma \mathrm{s}$ from the two-photons coming from $\Pi^{0}$ decays. 


\subsubsection{Event Trigger}

The signals produced by various subdetectors of CDF must be stored to an event-byevent basis for later analysis. By event here refers to each $p \bar{p}$ crossing. As typical instantaneous luminosity of $\approx 3 \cdot 10^{3} 1 \mathrm{~cm}^{-2} \mathrm{~s}^{-1}$ it is expected to have one protonantiproton interaction on average per bunch crossing. For the bunches separated by $396 \mathrm{~ns}$, the crossing occurs at rate of about $2.5 \mathrm{MHz}$. The time required to read out a single event from the entire detector is of order of $2 \mathrm{~ms}$ which is about 5000 times larger that the time between two consecutive bunch crossings. Another constraint on event acceptance rate comes from the data write-time, which is currently 10-20 ms for a typical event record size of $100-200 \mathrm{kB}$. It is obvious that most of the $p \bar{p}$ events can be recorded. Fortunately, most of these events are not interesting for physics analysis. Namely, most of the inelastic scattering events produced in $p \bar{p}$ collisions are of low momentum transfer, "minimum bias" events, with very little energy flow in the direction transverse to the beam. Events with large momentum transfer are more interesting for they probe physics at short distances, but are also less frequent, a factor of $10^{5}$ less than minimum bias events. A mechanism which selectively picks interesting events is needed. Commonly, this mechanism and its hardware are referred to as "trigger system".

Before permanently storing the data from a single event, the data pass three different levels of the CDF trigger system, as shown in Figure 3.10a. A more detailed block diagram is showing the selection logic is shown in Figure 3.10b. The maximum acceptance rate of Level-1 trigger is $50 \mathrm{kHz}$. The events passing $\mathrm{L} 1$ trigger requirements are further analyzed by Level-2 trigger whose acceptance rate is $300 \mathrm{~Hz} . \mathrm{i}++i$

Finally, the Level-3 trigger decides whether to accept the events for offline storage at the maximum possible writing speed of $75 \mathrm{~Hz}$. Since Level-1 and Level-2 systems have to make fast decisions they use only a part of the entire event data. Level- 1 and 


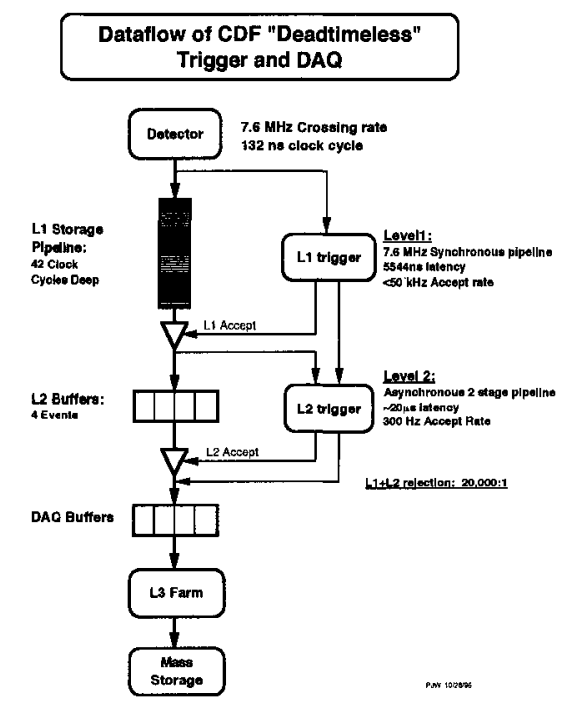

RUN II TRIGGER SYSTEM

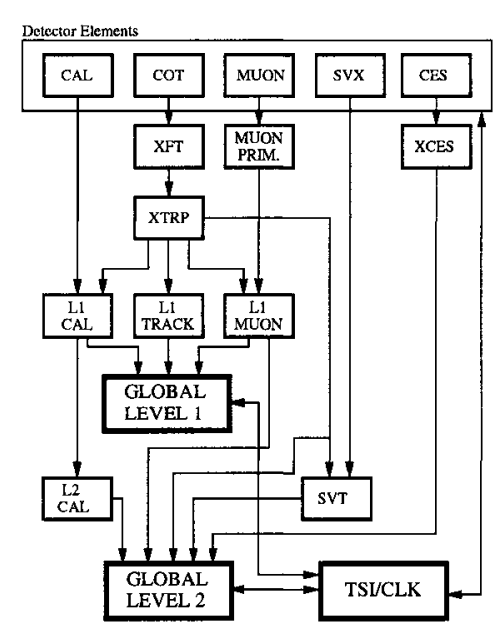

Figure 3.10: CDF data acquisition and trigger system in Run II

Level-2 are based purely on hardware (electronics) while L3 performs almost full event reconstruction using an appropriate software running on a computing farm.

As depicted in Figure ??b the inputs for Level-1 are the signals from the calorimeter detectors, the COT, and the muon detector. Up to 42 events (at 132 ns clock circle) can be stored in pipelined buffers where the data are stored for $5.5 \mu$ s and analyzed bu the three parallel synchronous streams. The first stream collects the information from the object and global calorimeter triggers. The object triggers fire off when the transverse energy deposited in individual calorimeter towers is above a certain threshold. The electron/photon trigger makes decisions based on the EM calorimeter energy, while jet trigger sets a threshold on the sum of EM and HAD energies in a tower. The global triggers compares the total energy from all towers and compares it to the reference value. All the thresholds and requirements on the number of objects can be preset 
at a desired value in the hardware of each detector subsystem. The second stream is responsible for finding stubs in the muon chambers. A novel part of the Level-1 trigger upgrade in Run II is the installation of the Extremely Fast Tracker (XFT) forming the third Level-1 trigger stream. The secret to the XFT's speed is that it reconstructs tracks in the transverse plain. Together with the extrapolation unit (XTRP) the tracks can be matched to clusters in EM calorimeter and muon chambers forming electron and muon primitives. The decision from above streams are then combined and the final decision if the event is accepted is made.

In case an event is accepted by Level-1 trigger all the information about this event is passed into one of four asynchronous event buffers of the Level-2 trigger system. In addition, the information from CES chambers and from $r-\phi$ strips of the SVX detector is transmitted to the Level-2 system. The Level-2 buffers are not able to hold more than four events. The average time to process one event sitting in the buffer is $20 \mu \mathrm{s}$, during which the trigger incurs dead time if the buffer is full. There are three hardware sybsystems part taking in the decision at Level-2: L2CAL, XSEC, and SVT. The L2CAL is the cluster finder which combines the energies collected by individual towers. Thus L2CAL provides. Thus L2CAL provides an approximate measurement of jet's $E_{T}$ and its direction. XCES uses a preset threshold to discriminate for signals from CES detector. This information combined with tracks extrapolated by XTRP is used for better track-to-cluster matching and therefore improved electron identification. The Silicon Vertex Tracker (SVT) reconstructs tracks in silicon vertex detector and determines the track impact parameter. When all the triggers of Level-2 are satisfied then the full detector readout is initiated.

After Level-1 and Level-2 triggers have made their decisions the events are transfered to the data acquisition system (DAQ) buffers. The system controlling the DAQ buffers, called the Event Builder, orders event fragments and sends them to a farm of conventional PCs serving as Level-3 triggers. The event fragments are assembled in a 


\section{Chapter 3. Experimental Apparatus}

block of data, eventrecord, suitable for analysis by CDF reconstruction software. The reconstruction of an event takes advantage of the full detector information and improved resolution not available at the lower trigger levels. If an event satisfies Level-3 requirements, the corresponding event record is sent and stored on a magnetic tape. Each event is marked by a number and by a number of the store, or the run, which the detector was taking data. During each run, the configuration of the detector is stable and recorded in a data base.

More information on CDF trigger system can be found elsewhere (ref TDR). Muon trigger is very important to this analysis and is discussed in the section below. 


\section{Chapter 4}

\section{Event Reconstruction}

The initial triage of events occurs at the online trigger level where a majority of background events is rejected. Physics data analysis requires even better purification of the selected events. The proper requirements are important to accept as many interesting events while reducing the number of background events to the minimum.

Before any meaningful analysis on the collected data happens, the information acquired from the detector and recorded on a permanent storage device must be reconstructed to represent the high level physical objects. At CDF, we the four most important high level objects are : electrons, muons, jets, and missing transverse energy. The reconstruction is performed in three steps. First, the hits in detectors subsystems are combined to form tracks, clusters, and stubs. Second, the cluster and tracking information are matched to the signature of known detectable particles. Third, the raw properties of the particle are corrected to represent the true four-momentum of the corresponding parton or lepton.

The reconstructed events are written in one or more streams according to the type and number of high level objects in the reconstructed event. In the sections below we describe reconstruction of the four basic high level object. 
Chapter 4. Event Reconstruction

\subsection{Lepton Identification}

The lepton identification is crucial for this analysis. Unlike electrons, muons and taus are unstable particles with average lifetimes $\tau_{\mu} \approx 2.2 \times 10^{-6}$ and $\tau_{\tau} \approx 2.91 \times 10^{-13}$ seconds. The relativistic muons pass through the detector without decaying, On contrary, the taus decay soon after they are produced. About $40 \%$ of the time tau will decay into a ligther lepton ( $e$ or $\mu$ ) and two neutrinos. The rest of the time the tau will decay hadronically producing particle jets. In this analysis we don't consider events with taus, but we concentrate on muons and electrons only.

\subsubsection{Electrons}

Electron is a light lepton with a mass of $0.511 \mathrm{MeV}$. At CDF electrons loose most of it energies in Bremsstrahlung process while moving through the lead absorbers. The photons produced at collisions of electrons with the absorber's nuclei have enough energy to convert into electron-positron pairs. Secondary particles collide with the nuclei producing tertiary particles, and so on, forming an electromagnetic (EM) cascade. Such a sower differs from the hadronic showers in jets by the number of tracks, shape and length. EM jets are identified by a high $p_{T}$ track in the drift chamber pointing to a EM calorimetry tower where substantial amount of energy was deposited. Typically, an electron jet deposits its energy in a single tower while a hadronic jet is much broader and it continues into hadronic calorimetry towers. The shape and a position of EM shower is measured by the shower maximum detector. Only electrons detected in the central calorimeter $(\mathrm{CEM}|\eta|<1.1)$. The full list of variables and the cuts used for identifying electrons is shown in Table 4.1

- $E_{T} \equiv E \sin \Theta \geq 20 \mathrm{GeV}$ The transverse energy of EM cluster $E_{T}$ is defined as a product of the total energy deposited in the EM cluster and $\sin \Theta$, where $\Theta$ is 
Chapter 4. Event Reconstruction

\begin{aligned} & Variable Cut \\ &$|\eta|<1 \\ & p_{T}>5 \mathrm{GeV} \\ & E_{T}>5 \mathrm{GeV} \\ &$ Fiduciality FidEleSmx ()$\neq 1$ andFidEleTrk ()$\neq 4 \\ & E_{H A D} / E_{E M}<0.055+0.00045 \times E \\ &$ number of axial segments $\geq 3 \\ &$ number of stereo segments $\geq 3 \\ &$ Isolation $\left(p_{T}>20\right)$ Cal_Iso $/ p_{T}<0.1 \\ &$ Isolation $\left(p_{T}<20\right)$ Cal_Iso $<0.1 \\ &$\hline\end{aligned}

Table 4.1: Electron ID cuts. Electron object must pass these cuts to be considered in this analysis

the angle of COT tracking pointing to the cluster.

- $p_{T} \geq 10 \mathrm{GeV} / c$ An electron in magnetic field moves along a helix, whose curvature depends on the electron's transverse momentum $p_{T}$. Due to differential response of COT to electrons and positrons, a small correction must be applied

$$
\frac{Q}{p_{T}}=\frac{Q}{p_{T}^{\text {raw }}}-0.00037-0.0011 \times \sin (\phi+0.28)
$$

where $\mathrm{Q}$ is the charge of the particle and $\phi$ is the track's azimuthal angle.

- $E / p \geq 2$ The absolute value of the electron's momentum as measured by COT track is required to be no less than $50 \%$ of the full $E$ measured in EM calorimeter. This requirement is justified by the situation where an electron loses a portion of its energy by emiting a photon while passing through the material at the inner COT radius. For the electrons with $p_{T}>50 \mathrm{GeV} / c$ the radiated photon will be almost certainly collinear to the electron's direction contributing to measured energy. For less energetic electrons the photon can end up in adjacent EM towers.

- $E_{h a d} / E_{E M} \leq 0.055+0.00045 E$ To avoid misidentification of a hadronic jet as electron, the fraction of energy $E_{H A D}$ deposited in the hadronic calorimeter is 
Chapter 4. Event Reconstruction

required to be a small fraction of the energy deposited in the electromagnetic calorimeter. The last term is there to compensate of the inefficiency of the cut for very high energy electrons that penetrate into the hadronic calorimeter.

- $L_{s h r}<0.2$ The energy deposited in the towers adjacent to the shower tower is expected to match the data from the test beam electrons. The quantity $L_{s h r}$ reflects how well the measured energy $E^{\text {meas }}$ matches the expected energy $E^{e x p}$ and it is given by:

$$
L_{s h r}=0.14 \sum \frac{E_{i}^{\text {meas }}-E_{i}^{\exp }}{\sqrt{(0.14 \sqrt{E})^{2}+\left(\Delta E^{e x p}\right)^{2}}}
$$

where $0.14 \sqrt{E}$ and $\Delta E^{e x p}$ are the uncertainties on the measured and expected energies. Typically, the sum is taken over two nearby towers in a cluster.

- $\chi_{\text {strip }}^{2}<10$ This quantity compares how well the CES shower profile in $r-z$ view compares with the same profile extracted from the test beam electrons. The fit is performed for each strip individually. This cut discriminates against wider clusters from fully or partially merged showers from $\Pi^{0}$ decays.

- $\left|z_{0}\right| \leq 60 \mathrm{~cm}$ The distance along the beam direction between the nominal interaction point $z=0$ and the point where the recosnrtucted track intersects the beam axis. The longitudinal spread of the $z$ component of the event vertex is subject to the gaussian distribution with $\sigma=26 \mathrm{~cm}$.

- Number of axial and stereo segments $\geq 3$ each. To ensure a good quality track associated with an electron candidate, it is required to pass through at least three axial and three stereo superlayers of the COT.

- Isolation $\leq 0.1$ This variable defines how much energy is deposited by other particles outside the EM cluster but inside the cone $\Delta R \leq 0.4$ and is given by

$$
\text { Isolation } \equiv \frac{E_{T}^{i s o}}{E_{T}^{E M c l u s t e r}}=\frac{E_{T}^{\text {cone }}-E_{T}^{E M c l u s t e r}}{E_{T}^{E M c l u s t e r}}
$$


Chapter 4. Event Reconstruction

where $E_{T}^{c o n e}$ and $E_{T}^{E M c l u s t e r}$ are the transverse energies corresponding to the cone and EM cluster. In fact, the Isolation is not an electron identification cut but rather a discriminator against non-isolated electrons produced in semi-leptonic decay inside the hadronic jets.

\subsubsection{Muon}

The muon is about 200 times more massive than the electron. It's only decay channel in SM is to electron. Although a non-stable particle, relativistic muon succeeds in traversing the detector without decaying. Its radiation length is much grater that that of electron, weakening its loss of energy due to Bremsstrahlung process. At CDF, muons are identified by a COT track matched to a stub of hits in the muon detector. The reconstructed muons are divided into three categories according to in which muon subdetector stubs are located: CMU, CMP and CMX. If the muon was reconstructed in both CMU and CMP chambers, then it is called CMUP muon.

The list of muon identification variables and the cuts imposed on their values are listed in Table 4.2. More details on the muon identification are given below.

- $p_{T} \geq 5 \mathrm{GeV} / c$ An electron in magnetic field moves along a helix, whose curvature depends on the electron's transverse momentum $p_{T}$. Due to differential response of COT to electrons and positrons, a small correction must be applied

$$
\frac{Q}{p_{T}}=\frac{Q}{p_{T}^{\text {raw }}}-0.00037-0.0011 \times \sin (\phi+0.28)
$$

where $Q$ is the charge of the particle and $\phi$ is the track's azimuthal angle. The cut value of 5 is motivated by the point at which muon ID and muon trigger efficiency reach a plateau as a function of $p_{T}$.

- $E_{E M} \leq \max (2,2+0.0115 \cdot(p-100)) \mathrm{GeV}$. This cut vetos muons with momentum greater than $100 \mathrm{GeV}$ that deposit energy greater than $2 \mathrm{GeV}$ in the $\mathrm{EM}$ 
Chapter 4. Event Reconstruction

$\begin{aligned} \text { Variable } & \text { Cut } \\ |\eta| & <1 \\ p_{T} & >5 \mathrm{GeV} \\ \text { Fiduciality (CMUP) } & \text { IsCMUFid()ANDisCMPFid() } \\ \text { (CMX) } & \text { IsCMXFid() } \\ \text { Stub(CMUP) } & \text { HasCMUStub()ANDHasCMPStub() } \\ (\text { CMX) } & \text { HasCMXStub() } \\ \left|\Delta_{C M U}\right| & <3 \mathrm{~cm} \\ \left|\Delta_{C M P}\right| & <5 \mathrm{~cm} \\ \left|\Delta_{C M X}\right| & <6 \mathrm{~cm} \\ \text { Matching }\left(p_{T}>20\right) & <3 \mathrm{~cm} \mathrm{OR} \chi_{C M U}^{2}<9 \\ \left|\Delta_{C M U}\right| & <5 \mathrm{~cm} \mathrm{OR} \chi_{C M P}^{2}<9 \\ \left|\Delta_{C M X}\right| & <6 \mathrm{~cm} \mathrm{OR} \chi_{C M X}^{2}<9 \\ E_{E M} & <\max (2,2+0.0115 \cdot(p-100)) \mathrm{GeV} \\ E_{H A D} & <\max (2,2+0.0115 \cdot(p-100)) \mathrm{GeV} \\ d_{0} & \leq 0.02(\leq 0.2) \mathrm{cm} \text { with (without } \mathrm{SVX}) \\ \text { numbering }\left(p_{T}<20\right) & \geq 3 \\ \text { number of stereo segments } & \geq 3 \\ \text { Isolation }\left(p_{T}>20\right) & \text { Cal_Iso/p } p_{T}<0.1 \\ \text { Isolation }\left(p_{T}<20\right) & \text { Cal_Iso }<0.1\end{aligned}$

Table 4.2: Muon ID cuts. Muon object must pass these cuts to be considered in this analysis

calorimeter. For muons of smaller momentum, this cut is corrected to make it more efficient.

- $E_{H A D} \leq \max (6,6+0.0280 \cdot(p-100)) \mathrm{GeV}$ This cut is hadronic analogy of the cut above.

- $|\Delta x|_{C M U} \leq 3 \mathrm{~cm},|\Delta x|_{C M P} \leq 5 \mathrm{~cm},|\Delta x|_{C M X} \leq 6 \mathrm{~cm}$ These cuts are imposed on the distance in $r-\phi$ plane between the muon track extrapolated in the relevant detector chambers and the muon stub segment. The CMU cut is loser than CMP and CMX because the CMU muon chambers are the closest of the three to the interaction point. The muons undergo the least amount of scattering before reaching CMU chambers. 
- $\rho_{C M X} \geq 140 \mathrm{~cm}$ The exit radius of a track from COT chamber must be smaller that $140 \mathrm{~cm}$. This cut disrciminates against tracks from displaced primary vertecies and are matched to CMX muons. with CMX muons.

- $\left|z_{0}\right| \leq 60 \mathrm{~cm}$ The distance along the beam direction between the nominal interaction point $z=0$ and the point where the reconstucted track intersects the beam axis. The longitudinal spread of the $z$ component of the event vertex is subject to the gaussian distribution with $\sigma=26 \mathrm{~cm}$.

- $d_{0} \geq 0.02(0.2) \mathrm{cm}$ with (without) SVX. The impact parameter $d_{0}$ is the distance of the closest approach of the reconstructed track and the $z$ axis. Only those muons which pass close to the vertex survive this requirement. Lots of cosmic muons from the kaon and pion decays are rejected by this cut. In this analysis we take an extra advantage of this cut in estimating our backgrounds. This is discussed later.

- Number of axial and stereo segments $\geq 3$ each. To ensure a good quality track associated with an electron candidate, it is required to pass through at least three axial and three stereo superlayers of the COT.

- Isolation $\leq 0.1$ This variable defines how much energy is deposited by other particles outside the EM cluster but inside the cone $\Delta R \leq 0.4$ and is given by

$$
\text { Isolation }=\frac{E_{T}^{i s o}}{E_{T}^{E M c l u s t e r}}=\frac{E_{T}^{c o n e}-E_{T}^{E M c l u s t e r}}{E_{T}^{E M c l u s t e r}}
$$

where $E_{T}^{c o n e}$ and $E_{T}^{E M c l u s t e r}$ are the transverse energies corresponding to the cone and EM cluster. In fact, the Isolation is not a muon identification cut but rather a discriminator against non-isolated muons produced in semi-leptonic decay inside the hadronic jets. 
Chapter 4. Event Reconstruction

\subsection{Jet Reconstruction}

Colliding protons and antiprotons have in them three valence quarks, a multitude of sea gluons and virtual quark-antiquark pairs. At high energies, such as $1 \mathrm{TeV}$, partons inside the hadrons can be considered as free particles. During the collisions they scatter and produce other hadrons of high transverse momenta as high as 600 $\mathrm{GeV}$. After scattering from the hadron, colorful parton very quickly interacts with the hadron remnant. If the potential energy of such interaction is high enough a pair of quarks will be produced and will join the free quark in making of a new colorless hadron. The newly formed hadron can further decay and cause further formation of colorless hadrons. The chain of such interaction continues till the energy of the secondary hadrons is low enough. This process is called hadronization. Its final result is a spray of collimated hadrons, a jet, in the direction of the initial parton momentum. The total energy of the jet is equal to the energy and is measured in the calorimeter.

In the calorimeter the jest of particles are recognized as clusters of energy deposited in adjacent towers. Since many jets can be produced in an event, a jet clustering algorithm is used to reconstruct the correct topology of the event. Here we describe a simple cone jet reconstruction algorithm. First, the seed towers with deposited transverse energy higher than $1 \mathrm{GeV}$ are chosen. Then, these towers are used as initial centroids around which all the towers within particular open angle $\Delta R=\sqrt{\Delta \phi^{2}+\Delta \eta^{2}}$ grouped into cluster. The towers belonging to the cluster and having transverse energy above $100 \mathrm{MeV}$ are used to calculate a new $E_{T}$-weighted centroid:

$$
\phi_{c} \text { entroid }=\frac{\sum_{i} E_{T, i} \phi_{i}}{\sum_{i} E_{T, i}} \phi_{c} \text { entroid }=\frac{\sum_{i} E_{T, i} \eta_{i}}{\sum_{i} E_{T, i}}
$$

where angular coordinate $\left(\phi_{i}, \eta_{i}\right)$ define the direction to the center of the $i^{\text {th }}$ calorimeter tower with transverse energy deposited in the tower $E_{T, i}$. The sum in Equations 3.1 and 3.2 is over all towers within a cone $\Delta R=0.4$. A new cone is drawn around the 


\section{Chapter 4. Event Reconstruction}

recalculated centroid and a new iteration is performed. The whole process is repeated till there is no change in the centroid's angular coordinates. Int the case when the two clusters are overlapping, they may be combined or not. The clusters are combined if the energy in the common towers is grater than or equal to $75 \%$ of the total energy in the smaller cluster, otherwise the overlap is assigned to the cluster with the closest centroid. In either case, the coordinates of the new centroids have to be recalculated.

Once all stable cones are identified, the following sums over the towers inside each cluster are used to calculate the raw four-momentum of the jets:

$$
p^{r a w} \equiv\left(E, p_{x}, p_{y}, p_{z}\right) \quad=\left(\sum E_{i} ; \sum E_{i} \sin \Theta_{i} \cos \phi_{i}\right)
$$

where $E_{i}$ is the energy deposited in the $i^{\text {th }}$ tower and $\Theta_{i}$ and $\phi_{i}$ are the polar and azimuthal angles defining the direction to the center of this tower.

It must be noted that the cone algorithm described above showed a satisfactory performance in the Monte Carlo, though it suffers from a few problems. Two "real" jets can be mistakenly merged into one cluster if the soft emission between them is above the seed threshold. On the other hand, the jets may not be reconstructed at all if their energy is evenly distributed among several towers where deposited $E_{T}$ is less than the threshold. Thus, the number of reconstructed jets depends on empirically determined parameters such as jet cone size and seed threshold.

On must adjust the raw jet momentum, to correct for things like detector's imperfections or physics effects. For example, one kind of correction is due to the cracks between the towers and the dependence of calorimeter's response on the rapidity. The physical reasons have to do with multiple interactions and deposition of particles' energy outside the cone. Seven additive and multiplicative levels of corrections are applied to the raw energy in the following way:

$$
E_{t}=\left(E_{T}^{\text {raw }} \times f_{\text {rel }} \times f_{\text {time }} \times f_{\text {scale }}-E_{T}^{M I}\right) \times f_{a b s}-E_{T}^{U E}+E^{O O C} .
$$


The description of each factor is presented below.

- The relative correction ( $f_{\text {rel }}$ is responsible for adjusting the responses from the calorimeter towers at different pseudorapidities making them uniform function of $\eta$. The scale factor is determined by comparing the transverse energy balance in di-jet events where one jet is measured in well-calibrated central region far from the detector cracks and the other is lying outside the $0.2<|\eta|<0.6$ region.

- The time dependent correction $\left(f_{\text {time }}\right.$ is responsible for justifying the calorimeter responses due to their deterioration in time, e.g., aging of phototubes.

- The scale correction $\left(f_{\text {scale }}\right)$ accounts for differences in jet energy measurements in Run I and Run II. The photon energy deposited in the EM calorimeter is measured with high precision. The scale factor is determined from the photonjet balance:

$$
f_{\text {scale }}=\frac{1+\left\langle f^{\text {RunI }}\right\rangle}{1+\left\langle f^{\text {RunII }}\right\rangle},
$$

where $f^{R u n I, I I}=\frac{p_{T}^{\text {jet }}-p_{T}^{\gamma}}{p_{T}^{\gamma}}$

- The correction for multiple interactions $\left(E_{T}^{M I}\right)$ is required if more than one interaction per bunch crossing happens. The events can have an extra overlapping minimum bias event. The energy from these events may fall into the jet clustering cone and must be subtracted from the raw value. The number of additional vertecies is used to estimate the number of soft $p \bar{p}$ scatterings. The additional energy per interaction $E_{T}^{M I}$ in the jet cone of the given size $\Delta R=0.4$ is estimated by measuring the transverse energy of a random cone as a function of the number of the reconstructed vertecies in the minimum bias event.

- The absolute energy correction $f_{a b s}$ scales the measured jet energy to the energy of the original parton as it is defined by the Monte Carlo simulation. The true 


\section{Chapter 4. Event Reconstruction}

parton energy is usually underestimated because of nuclear absorption, energy loss in uninstrumented region and non-linear response to the neutral and charged pions. The multiplicative factor $f_{a b s}$ is determined by scaling the sum of $p_{T}$ 's of the particles within the cone of same size around the parton direction in Monte Carlo by the $E_{T}$ of the jet.

$$
f_{a b s}=\left\langle\frac{\sum p_{T, i}^{M C p a r t i c l e}}{E_{T}^{j e t}}\right.
$$

- The underlying event correction $\left(E_{T}^{U E}\right)$ is defined by activities that are not directly related to the physical process of interest. The sources of such parasitic activities include the interactions, also referred as underlying events, of spectator partons and proton-antiproton remnants. This value, usually small, is measured from the minimum bias events with only one vertex.

- The out-of-cone $E^{O O C}$ is conditioned by the fact that not all particles produced by the initial parton lie inside the jet cone. The usual cause of such energy leakage is the soft gluon radiation. To account for the escaped particles, the certain amount of energy needs to be added to the energy of the jet cluster. An exact value of $E^{O O C}$ is determined from the Monte Carlo simulations by calculating the average difference between the actual parton transverse momentum and the sum of the transverse momenta of all daughter particles lying inside the jet cone:

$$
E^{O O C}=p_{T}^{p a r t o n}-\sum p_{T, i}^{M C p a r t i c l e}
$$

The application of the corrections to the raw jet energy heps to estimate the true energy of the parton as accurate as possible. The total systematic uncertainty on the jet $E_{T}$ is determined from the individual uncertainties assigned to each correction level. The overall uncertainty depends on the jet $E_{T}$ value and varies form $5 \%$ to $12 \%$. The central jets are measured slightly better than the jets in the plug region. 


\subsection{Missing Energy}

Neutrinos are chargless particles which participate only in the weak interactions with other particles. An impact from the neutrino passing through the detector material is too small that it cannot be detected. The presence of the neutrino(s) in the event can be deduced from the imbalance in the total observed transverse energy. The initial transverse momentum of the system of interacting partons is negligible and so must be the vector sum of all final particle momenta projected on the transverse plane. If the latter is not consistent with zero, the missing transverse energy $\left(E_{T}\right)$ is introduced to restore the balance according to the total momentum conservation:

$$
\mathbb{E}_{T}=-\sum \operatorname{vecp}_{T, i}
$$

Since the CDF is sealed around the $z$ axis the sum on the right side of Eqautaion ?? can be calculated over all detected particles, and thus the missing transverse energy can be associated with the neutrino's transverse momentum. Unlike with the transverse momentum we cannot make any assumptions about the initial longitudinal components of the interacting partons. Many particles with the very high pseudorapidity values are not detected, so neutrino's $z$ component of the momentum cannot be measured.

The raw value of ${\overrightarrow{E_{T}}}^{\text {raw }}$ is estimated very fast at the trigger level by summing the transverse energies of all calorimeter towers:

$$
\left.{\overrightarrow{E_{T}}}^{r} a w=-\sum\left(E_{i} \sin \Theta_{i}\right) \overrightarrow{(n}\right)_{i} \equiv-\sum E_{T, i} \vec{n}_{i}
$$

where $E_{i}$ is the energy deposited in the $i^{t h}$ tower, $\Theta_{i}$ is the polar angle of the vector pointing from the event vertex to the center of the $i^{\text {th }}$ tower, and $\vec{n}_{i}$ is the component of that vector lying in the plane perpendicular to the beam direction. If the azimuthal angle of ${\overrightarrow{E_{T}}}^{r}$ aw is $\phi_{\vec{E}_{T}}$ then the $x$ and $y$ components are given by

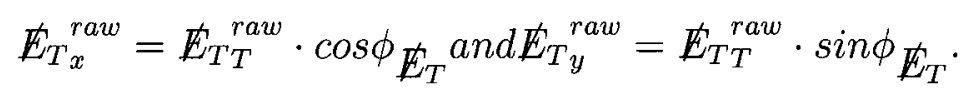




\section{Chapter 4. Event Reconstruction}

All towers with the pseudorapidity $\left|\eta_{i}\right|<3.6$ and the deposited energy $E_{i}>100 \mathrm{MeV}$ are taken into account in Equation 3.15.

The measurement of missing energy is corrected to account for imperfections of the detector. There are two major correction to the $\mathbb{E}_{T}$. One is a correction to jet energies and the other one is correction to the muon's energies. The former subtracts the corrected jet energies $\vec{E}_{T, j}^{\text {corr }}$ and adds uncorrected jet energies $\vec{E}_{T, j}^{\text {corr }}$ to $\vec{E}_{T}^{\text {raw }}$. The later correction subtracts the muon $p_{T} \mathrm{~s}$ which are more accurate measurements of muons' transverse energies and and adds muon energies measured in EM calorimeter $\vec{E}_{T, \mu}$ in the expression form ${\overrightarrow{E_{T}}}^{\text {raw }}$. The both correction can be summarized as

$$
\vec{E}_{T}=\not E_{T x}^{r a w}-\left(\sum \vec{E}_{T, j}^{c o r r}-\sum \vec{E}_{T, j}^{u n c o r r}\right)-\left(\sum \vec{E}_{T, \mu}-\sum \vec{E}_{T, \mu}\right)
$$

The uncertainty on the missing transverse energy primarily comes from the uncertainties on jet energy and muon momentum measurements. 


\section{Chapter 5}

\section{Measurement of Low $p_{T}$ Muon Trigger Efficiencies}

At CDF RunII, medium $p_{T}$ dimuon trigger efficiencies have been measured using muon calibration sample, [19], and low $p_{T}$ muon trigger efficiencies have bean measured using $\mathrm{J} / \Psi$ sample, [20]. In both cases, data processed with cdfsoft2 version 5.3.1 were used. Dilepton data, amounting to $700 \mathrm{pb}^{-1}$, has been processed with version 6.1.1 of $c d f s o f t 2$ software. It is, therefore, necessary to measure muon trigger efficiency for these data. This is the first measurement of SUSY DILEPTON muon trigger efficiencies using the SUSY DILEPTON dataset itself.

\subsection{Trigger Efficiency and Trigger Paths}

Muon trigger efficiencies we measured can be used to derive total trigger efficiencies, $\left(\epsilon_{t}\right)$, of the following SUSY DILEPTON paths:

- DIMUON_CMUCMU4 
Chapter 5. Measurement of Low $p_{T}$ Muon Trigger Efficiencies

- DIMUON_CMU4CMX4

- DIMUON_CMUPCMUP4

- DIMUON_CMUP4CMX4

where trigger efficiency is measured relative to offline CdfMuon.

\subsubsection{Total Path Trigger Efficiency}

Total path trigger efficiency is given by the product of L1, L2, and L3 trigger efficiencies.

$$
\begin{gathered}
\epsilon_{t}=P(\mathrm{~L} 1, \mathrm{offl} \mid \mathrm{off}) \times \mathrm{P}(\mathrm{L} 1, \mathrm{~L} 2, \mathrm{offl} \mid \mathrm{L} 1, \mathrm{off}) \times \mathrm{P}(\mathrm{L} 1, \mathrm{~L} 2, \mathrm{~L} 3, \mathrm{offl} \mid \mathrm{L} 1, \mathrm{~L} 2, \mathrm{offl}) \\
\epsilon_{t}=\epsilon_{L 1} \times \epsilon_{L 2} \times \epsilon_{L 3}
\end{gathered}
$$

\section{Dimuon Trigger Efficiency}

SUSY DILEPTON dimuon trigger paths consist of dimuon triggers at L1, L2, and L3. Dimuon trigger efficiency, $\epsilon_{i}\left(\mu_{1}, \mu_{2}\right)$, at any of the three trigger levels $i$ can be expressed as a product of single-leg muon trigger efficiencies at that trigger level $i$ :

$$
\epsilon_{i}\left(\mu_{1}, \mu_{2}\right)=\epsilon_{i}\left(\mu_{1}\right) \times \epsilon_{i}\left(\mu_{2}\right)
$$

as discussed in [23].

\section{Offline CdfMuon, Trigger Requirements}

We impose the following cuts on offline CdfMuon to purify the data sample,

- Offline CdfMuon: 
Chapter 5. Measurement of Low $p_{T}$ Muon Trigger Efficiencies

- track: $\left|z_{0}\right|<60 \mathrm{~cm},\left|\Delta z_{0}\right|<4 \mathrm{~cm}, Q_{1}+Q_{2}=0,\left|d_{0}\right|<0.2$ or $\left|d_{0}\right|<0.02$ $\mathrm{cm}$ if SVX hits available.

It is important to be able to determine if an offline CdfMuon fired a given trigger. To do so we impose following requirements:

- L1 requirement

- L1 trigger in list of passed triggers at L1 in PhysicsTriggerTable.

- match offline muon's stub to TCMD muon primitive-track match info. Match to $2.5^{\circ}$ in $\phi$. [30].

- matching done using stnTrig class, developed by UNM group [29].

- L2 requirement

- L2 trigger in list of PASSED triggers at L2 in PhysicsTriggerTable.

- match offline muon's stub to TCMD muon primitive-track match info. Match to $2.5^{\circ}$ in $\phi$.

- L3 requirement

- L3 trigger in list of PASSED triggers at L3 in PhysicsTriggerTable.

- L3 to Offline Track matching $\left(\Delta R<0.1 \mathrm{rad}, \Delta Z_{0}<1 \mathrm{~cm}\right)$.

First, we identify the trigger leg using the method described above. The second leg is then unbiased, an example of which can be seen in ??.

Once we have the sample of unbiased muons, then we apply additional cuts: 
Chapter 5. Measurement of Low $p_{T}$ Muon Trigger Efficiencies

- Minimal track's distance from the origin in $z$ direction at any axial superlayer of COT, $z M i n_{-} A x S L$, must be greater than $1.5 \mathrm{~cm}$. In COT, at about $|z|<1.5$ there are glass spacer. ${ }^{1}$ COT hit efficiency close to the spacers is very small. Since XFT requires 11 of 12 possible hits L1 tracking efficiency is significantly effected by this cut [31], [20].

- Due to L2 requirement we impose tracks to be more than 1.25 deg separated in $\phi$ (i.e. $\left.\Delta \phi_{S L 6}>1.25 \mathrm{deg}\right)[20]$.

- We don't consider muons that are less than two stacks apart in the muon chambers. Since each stack is $1.25 \mathrm{deg}$ in $\phi$ and a single muon can have hits in two stacks at most, we eliminate undesired muon pairs by imposing $\Delta \phi_{s t u b}>7.5 \mathrm{deg}$, if the muons are found in the same side of a single muon detector (East or West).

\subsection{2 ediloh Dataset}

Dataset edil0h collected with three trigger datasets (SUSY_DILEPTON_ 2_v-5, 3_v-1, 3_v-2), which were used in total of eight different PHYSICS tables $(00,01,02,04,05$, $06,07,08)$.

\begin{tabular}{ccccc}
\hline$\#$ & low, high run \# & SUSY_DILEPTON_ & PHYSICS_3_ & luminosity $\left(p b^{-1}\right)$ \\
\hline 1 & 190893,200272 & $2 \_v-5$ & $00,01,02$ & 332 \\
2 & 200076,202814 & $3 \_v-1$ & $04,05,06$ & 32 \\
3 & 201456,203489 & 3_v-2 & $06,07,08$ & 122 \\
\hline
\end{tabular}

Table 5.1: Three groups of runs for which different SUSY_DILEPTON triggers were used. Second column gives lowest and highest run number, third column is version of SUSY_DILEPTON trigger dataset used, fourth column is version of PHYSICS_3 table used, fifth column is integrated luminosity collected. Note that run ranges are not contiguous.

\footnotetext{
${ }^{1}$ Spacers serve to maintain constant COT layer separation.
} 
Chapter 5. Measurement of Low $p_{T}$ Muon Trigger Efficiencies

For group of runs \# 1, listed in the first row of Table 5.1, SUSY_DILEPTON_2_v-5 triggers were used, which are shown in Table 5.2 .

We used dimuon paths (\#15-16) for the calculation of the following single-leg triggers:

- L1_CMU1.5_PT1.5

- L2_CMU6_PT4

We used dimuon paths (\#17-18) for the calculation of the following single-leg triggers:

- L1_CMX1.5_PT2

- L2_CMX1.5_PT4

We used single electron path (\#19 ELECTRON_4_LOOSE_v-3) and electron-muon path (\#2 CEM4_CMU4_v-2) for the calculation of L3 single-leg trigger:

\section{- L3 CMU4}

We used single electron path (\#19 ELECTRON_4_LOOSE_v-3) and electron-muon path (\#4 CEM4_CMU4_V-2) for the calculation of L3 single-leg trigger:

\section{- L3 CMX4}

For groups of runs \# 2 and \# 3, listed in the second and the third row of Table 5.1, SUSY_DILEPTON_3_v-1 and SUSY_DILEPTON_3_v-2 triggers were used, which are shown in table 5.3 and 5.4. In these trigger sets CMU trigger paths were substituted 
Chapter 5. Measurement of Low $p_{T}$ Muon Trigger Efficiencies

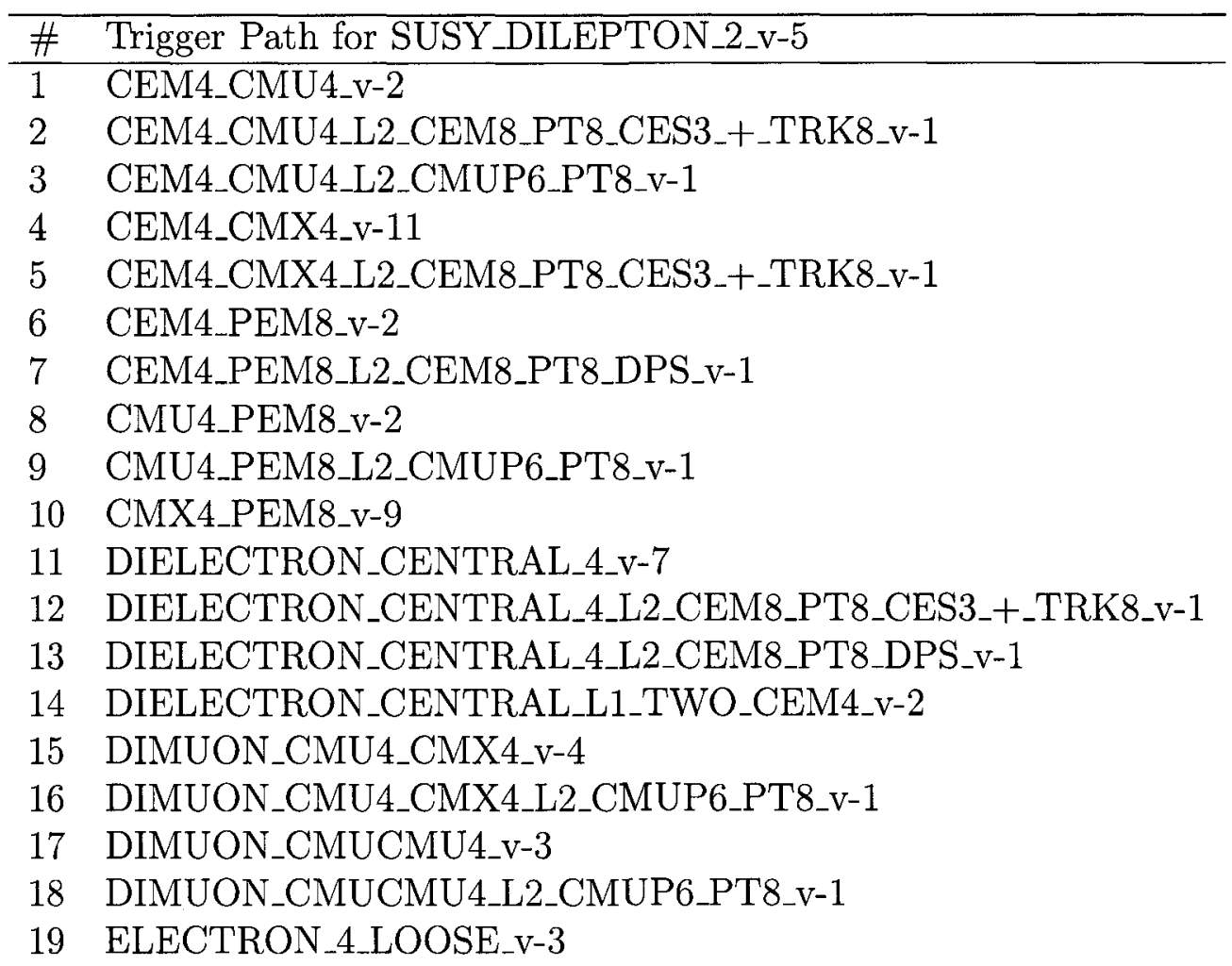

Table 5.2: Trigger paths in SUSY_DILEPTON_2_v-5. Note the presence of single electron path. Also note the presence of DIMUON paths with single muon triggers at L2 (\# 16 and \# 18)

by CMUP trigger paths. The key difference between the two paths is a change at L2, from L2 TWO_CMU6_PT4 for CMU paths to L2 TWO_CMUP6_PT4 for CMUP paths. Both paths have common L1 trigger, L1 TWO_CMU1.5_PT1.5. The measurement of L2 CMUP6_PT4 single-leg trigger efficiency is not described in the current version of this document.

\subsubsection{L1 and L2 Single-Leg Trigger Efficiency}

As described in Section 2.1, to obtain dimuon trigger efficiency we must first determine single-leg muon trigger efficiencies. In the following subsections we describe how single- 
Chapter 5. Measurement of Low $p_{T}$ Muon Trigger Efficiencies

\begin{tabular}{ll}
\hline$\#$ & Trigger Path for SUSY_DILEPTON_3_v-1 \\
\hline 1 & CEM4_CMUP4_v-8 \\
2 & CEM4_CMX4_v-11 \\
3 & CEM4_PEM8_v-2 \\
4 & CMUP4_PEM8_v-7 \\
5 & CMX4_PEM8_v-9 \\
6 & DIELECTRON_CENTRAL_4_v-7 \\
7 & DIMUON_CMUP4_CMX4_v-8 \\
8 & DIMUON_CMUPCMUP4_v-5 \\
9 & ELECTRON_CENTRAL_16_v-1 \\
10 & ELECTRON_CENTRAL_8_+_TRACK8_v-1 \\
11 & MUON_CMUP15_v-1 \\
12 & MUON_CMUP8_+_TRACK8_v-1 \\
13 & MUON_CMX15_v-1 \\
14 & MUON_CMX8_+_TRACK8_v-1
\end{tabular}

Table 5.3: Trigger paths in SUSY_DILEPTON_3_v-1. Note the presence of CMUP paths and the absence of CMU paths.

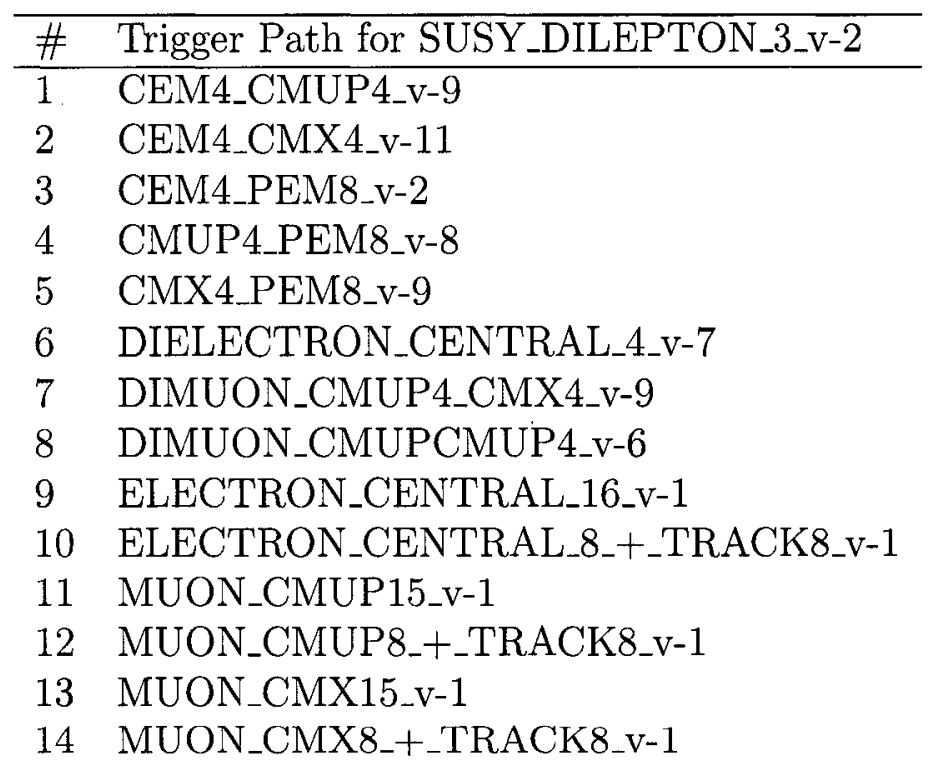

Table 5.4: Trigger paths in SUSY_DILEPTON_3_v-2. Note the presence of CMUP paths and the absence of CMU paths. 
Chapter 5. Measurement of Low $p_{T}$ Muon Trigger Efficiencies

leg CMU and CMX trigger efficiencies were measured.

\section{Dataset}

For the measurement of L1 and L2 single-leg muon trigger efficiency we collected data from runs in row \#1 of Table 5.1 skimmed by dimuon path with single L1 and L2 CMUP triggers (L3 path DIMUO4_CMUCMU(CMX)4_L2_CMUP6_PT8). This path contains a single L2 trigger (L2_CMUP6_PT8), and a single L1 trigger (L1_CMUP6_PT_4)

\section{Algorithm and Calculation of Efficiency}

In the skimmed dataset of dimuon events we reconstruct resonances $J / \psi, \Upsilon$, and $\mathrm{Z}$. We identify at least one of the two muons to have fired the L2 CMUP6_PT8 skimming trigger. We label this leg as trigger leg and the other leg, regardless if it fired the skimming trigger, as other leg and then we test if the other muon triggered L1 $\mathrm{CMU}(\mathrm{CMX}) 1.5$. We use the following mass windows cuts for the resonances: $\mathrm{J} / \Psi$ $(2.7-3.4 \mathrm{GeV} / c), \Upsilon(8-11 \mathrm{GeV} / c), Z(60-120 \mathrm{GeV} / c)$ We use the side-band subtraction to correct for possible residual fake muons.

- For $J / \psi$, we define peak signal (sig) to be $3.0<$ mass $<3.2$, and we define side bands $(\mathrm{sb})$ to be $2.8<$ mass $<3.0$, and $3.2<$ mass $<3.4$.

- For $\Upsilon$, we define peak signal (sig) to be mass $\in(9.3,9.6)$ and $(9.9,10.4)$ and we define side bands $(\mathrm{sb})$ to be mass $\in(8.6,9.1),(9.7,9.8)$, and $(10.6,10.8)$.

- For $Z$, we define peak signal (sig) to be mass $\in(80,100)$, and we define side bands $(\mathrm{sb})$ to be mass $\in(60,70)$ and $(110,12)$.

Since the skimming trigger L2 CMUP6_PT8 has harder cuts than those of the triggers whose efficiency we are measuring, a special care must be taken in the calculation 


\section{Chapter 5. Measurement of Low $p_{T}$ Muon Trigger Efficiencies}

of the trigger efficiency. Let $\epsilon_{l}$ be the single-leg efficiency of the low pt trigger which we are trying to measure, and let $\epsilon_{h}$ be the single-leg efficiency of the high pt trigger that we used to select the dimuon events. Low pt trigger has softer cuts than the high pt trigger, and let's express it as $\epsilon_{l}=\epsilon_{h}+\alpha$, where $0<\alpha<1$.

Given $\mathrm{N}$ dimuon events in the original dataset, then

- $N_{h h} \equiv$ number of events with both legs passing high trigger $=N \epsilon_{h}^{2}$.

- $N_{h \alpha} \equiv$ number of events with one leg passing high trigger and the other leg passing low but not high trigger $=2 N \epsilon_{h} \alpha$.

- $N_{h \bar{\alpha}} \equiv$ number of events with one leg passing high trigger and the other leg not passing low trigger $=2 N \epsilon_{h}\left(1-\epsilon_{l}\right)$.

Further, let's define

- $A \equiv 2 N_{h h}+N_{h \alpha}+N_{h \bar{\alpha}}$

$$
\begin{gathered}
=2 N \epsilon_{h}^{2}+2 N \epsilon_{h}\left(\epsilon_{l}-\epsilon_{h}\right)+2 N \epsilon_{h}\left(1-\epsilon_{l}\right) \\
=2 N \epsilon_{h}
\end{gathered}
$$

- $B \equiv 2 N_{h h}+N_{h \alpha}$

$$
\begin{gathered}
=2 N \epsilon_{h}^{2}+2 N \epsilon_{h}\left(\epsilon_{l}-\epsilon_{h}\right) \\
=2 N \epsilon_{h} \epsilon_{l}
\end{gathered}
$$

The single-leg muon low trigger efficiency is then

$$
\epsilon_{L 1}^{\mu}=\epsilon_{l}=\frac{A}{B}
$$

- This applies for both L1 and L2 trigger efficiency measurements. 
Chapter 5. Measurement of Low $p_{T}$ Muon Trigger Efficiencies

For the calculation of CMU L1 and L2 efficiencies we included all the wedges of the CMU detector, i.e. the entire CMU detector. However, we applied the official Good Run List version 11[32], compiled with option $(0,0,3,1)^{2}$ and with option $(0,0,4,1)^{3}$ for the measurement of CMU and CMX trigger efficiencies, respectively. In the case of CMU trigger efficiencies measurement, we separately considered $J / \psi$ and $\mathrm{Z}$ resonances combined and $\Upsilon$ and $\mathrm{Z}$ resonance combined. In the case of CMX trigger efficiency measurement, we combined all three resonances due to lower statistics. We measured trigger efficiencies as functions of $p_{T}$, $\phi$ (i.e. wedge), detector $\eta$. The plots of efficiency versus $p_{T}$ we try to fit with a function of three parameters,

$$
f(x)=\frac{p_{0}}{1+\exp \left(p_{1}-x / p_{2}\right)}
$$

where $x$ is $p_{T}$.

\footnotetext{
${ }^{2} \mathrm{CMU}$ and CMP detectors were required to be performing well.

${ }^{3} \mathrm{CMU}, \mathrm{CMP}$ and CMX detectors were required to be performing well.
} 
Chapter 5. Measurement of Low $p_{T}$ Muon Trigger Efficiencies

\section{CMU L1 and L2 from $J / \psi$ and Z}

Results for CMU L1 and L2 efficiencies, obtained from $J / \psi$ and Z resonances, are shown in figures 5.3 and 5.4. Same type of plots but without $z M i n A x S L^{4}$ cut applied to the testing muon, obtained from $J / \psi$ and $\mathrm{Z}$ resonances, are shown in figures 5.5 and 5.6. Imposing $z \operatorname{Min} A x S L$ cut improves L1 CMU trigger efficiency by about 3 percent, and removes $\eta$ dependence of the efficiency, as it can be seen from the plots in figures 5.3 and 5.5 .
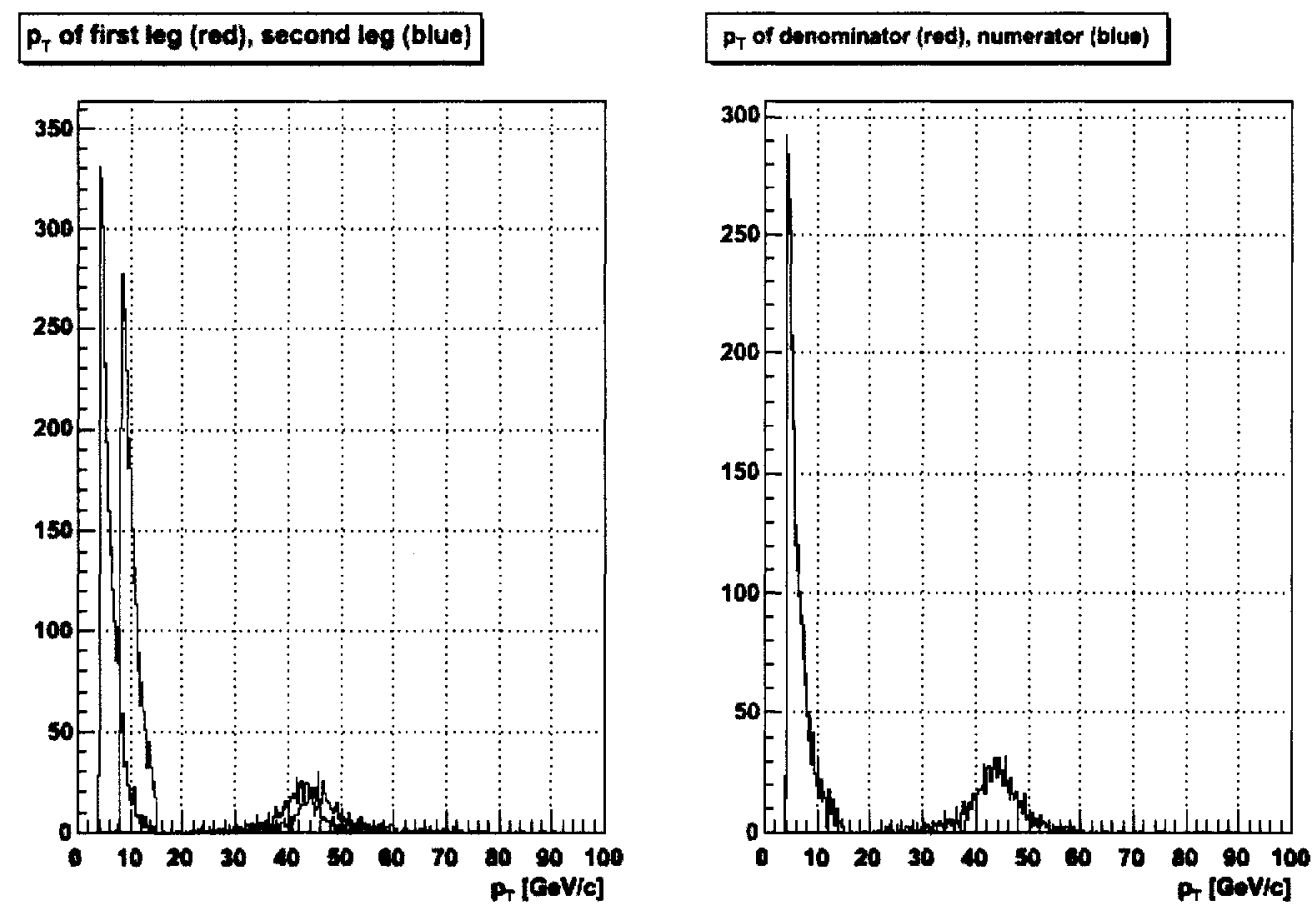

Figure 5.1: $J / \psi$ and Z Left: $p_{T}$ of trigger leg (red) and other leg (blue) leg. Right: $p_{T}$ of other leg, numerator (blue), denominator (red)

\footnotetext{
${ }^{4}$ Described in section 2.1 .2
} 
Chapter 5. Measurement of Low $p_{T}$ Muon Trigger Efficiencies

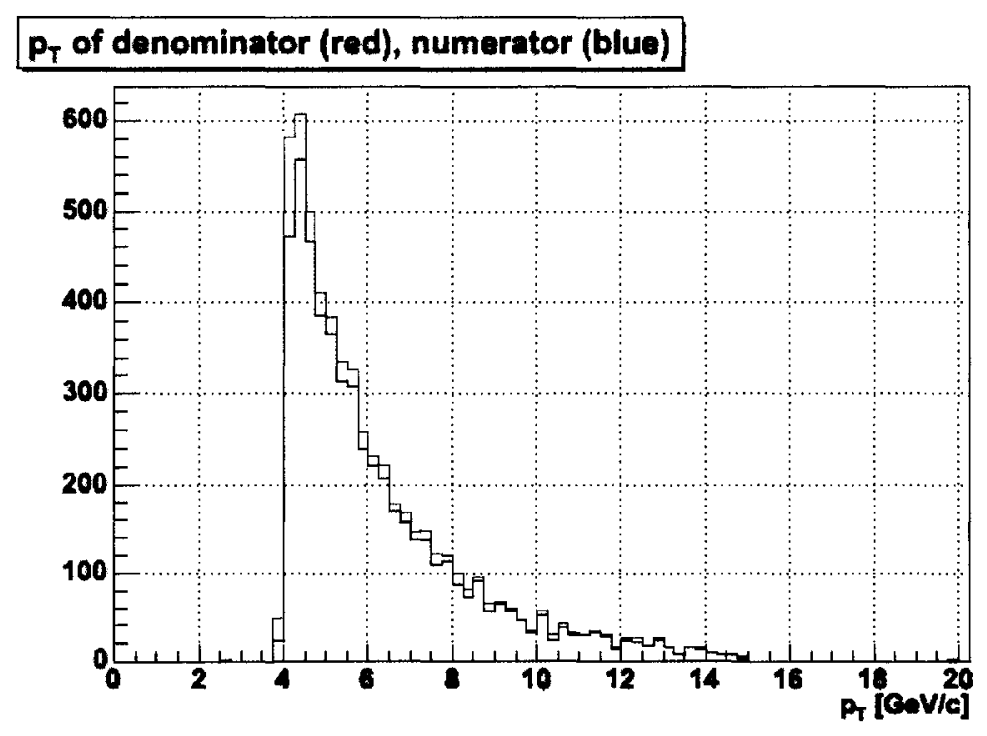

Figure 5.2: Zoom-in of $p_{T}$ of denominator (OTHER leg) and numerator. No obvious shoulder at $p_{T}=8 \mathrm{GeV}$, indicating bias by L2 trigger used for our event selection.

\section{CMU L1 and L2 from $\Upsilon$ and $\mathrm{Z}$}

Results for CMU L1 and L2 efficiencies, obtained from $\Upsilon$ and Z resonances, are shown in figures 5.8 and 5.9. Same type of plots but without $z M$ inAxSL cut applied to the testing muon, obtained from $J / \psi$ and $\mathrm{Z}$ resonances, are shown in figures 5.10 and 5.11 . As it was the case with $J / \psi$ and $\mathrm{Z}$ resonances and discussed in the previous subsection, imposing $z \operatorname{Min} A x S L$ cut improves L1 CMU trigger efficiency by about 3 percent, and removes $\eta$ dependence of the efficiency, as it can be seen from the plots in figures 5.8 and 5.10 . 
Chapter 5. Measurement of Low $p_{T}$ Muon Trigger Efficiencies
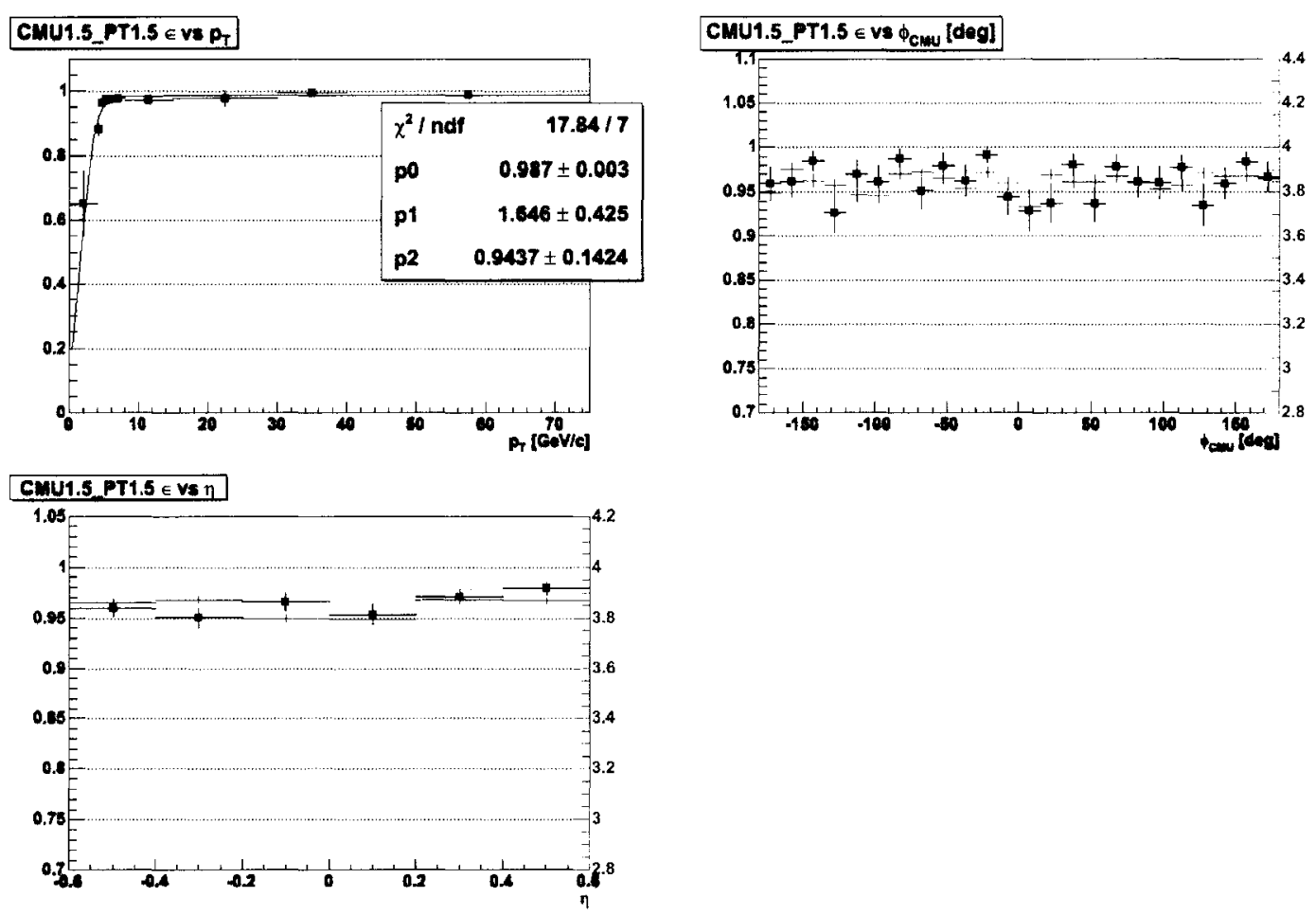

Figure 5.3: $J / \psi$ and Z After SB subtraction, single-leg L1 efficiency vs $p_{T}$ (top left), vs $\phi$ (top right), vs $\eta$ (bottom). A fit function shown in blue (top right). Number of CMU hits shown in red (top right and bottom left). For $p_{T}>6 \mathrm{GeV} / c$ efficiency becomes flat. Efficiency is approximately flat as a function of $\phi$ and $\eta$.

5.11. 
Chapter 5. Measurement of Low $p_{T}$ Muon Trigger Efficiencies
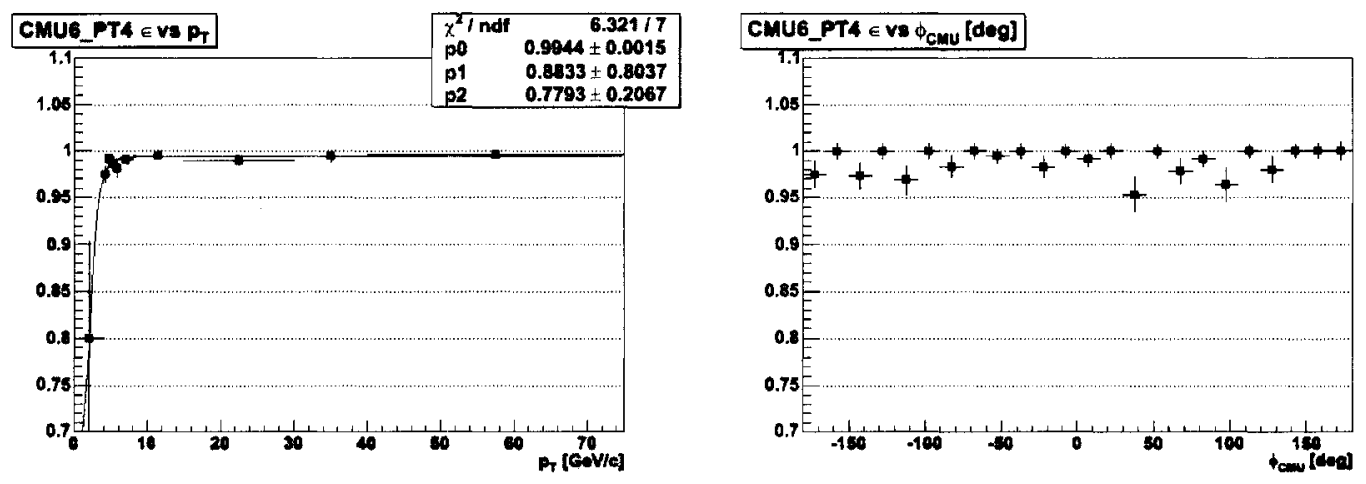

Figure 5.4: $J / \psi$ and Z After SB subtraction, single-leg L2 efficiency vs $p_{T}$ (top left), vs $\phi$ (top right), vs $\eta$ (bottom). A fit function shown in blue (top right). For $p_{T}>6$ $\mathrm{GeV} / c$ efficiency becomes flat. Efficiency is approximately flat as a function of $\phi$ and $\eta$.

\section{L1 and L2 from $J / \psi, \Upsilon$, and Z for East Arch Only}

When measuring CMX trigger efficiencies in the East CMX Arch we did not impose cut $Z$ MinAxSL. It had negligible effect on the results. We also did not include neither the Keystone nor the Miniskirt. Results for CMX L1 and L2 efficiencies in the East CMX Arch, obtained from $J / \psi, \Upsilon$ and $\mathrm{Z}$ resonances, are shown in figures 5.12, 5.13 and 5.14 
Chapter 5. Measurement of Low $p_{T}$ Muon Trigger Efficiencies
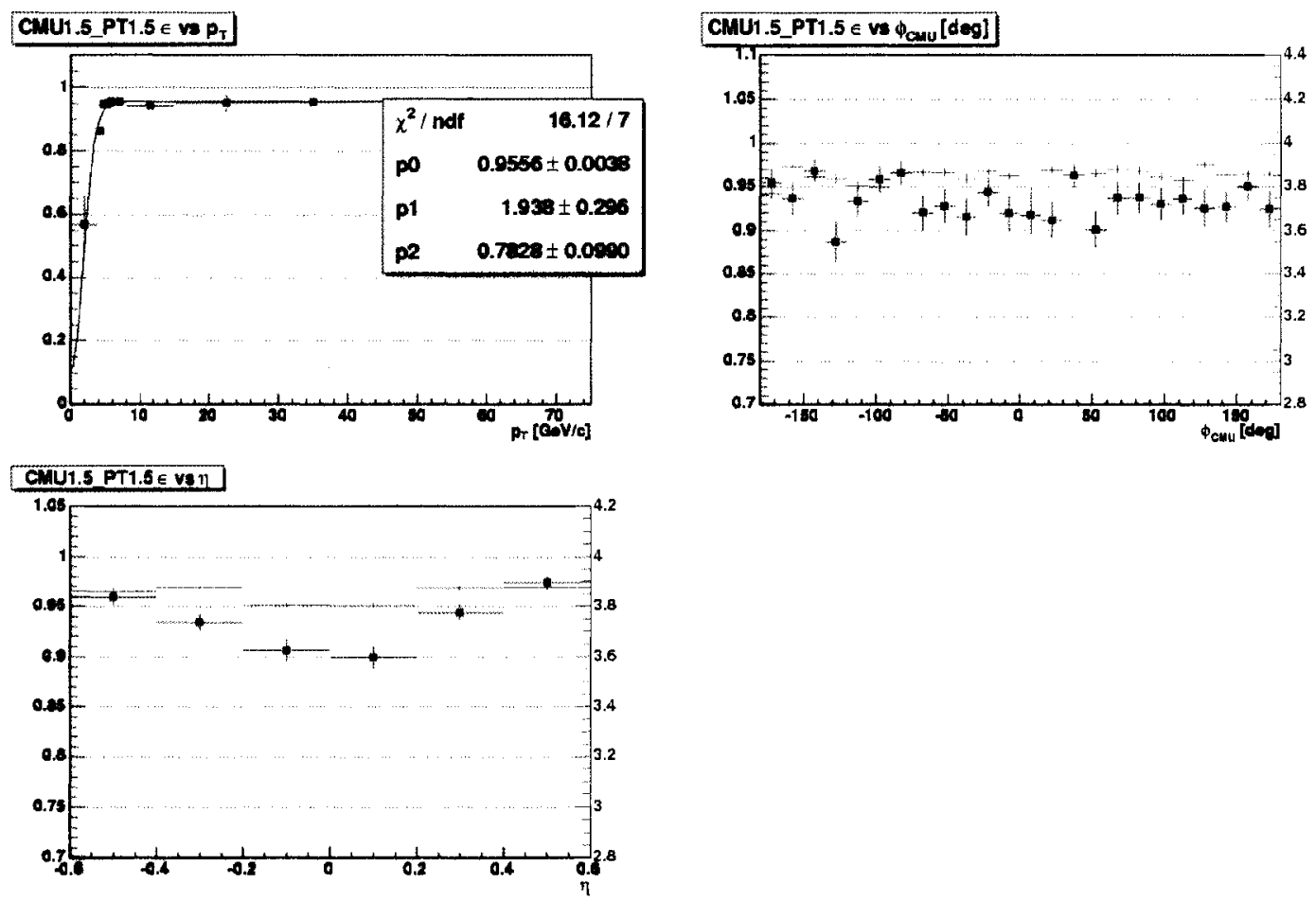

Figure 5.5: $J / \psi$ and Z After SB subtraction and $z M$ in $A x S L$ cut not applied, single-leg L1 efficiency vs $p_{T}$ (top left), vs $\phi$ (top right), vs $\eta$ (bottom). A fit function shown in blue (top right). Number of CMU hits shown in red (top right and bottom left). The absence of $z \operatorname{Min} A x S L$ cut lowers the efficiency and introduces a significant $\eta$ dependence.

CMX L1 and L2 from $J / \psi, \Upsilon$, and Z for West Arch Only

When measuring CMX trigger efficiencies in the West CMX Arch we did not impose cut $Z$ Min $A x S L$. It had negligible effect on the results. We also did not include neither the Keystone nor the Miniskirt. Results for CMX L1 and L2 efficiencies in the Wast CMX Arch, obtained from $J / \psi, \Upsilon$ and $\mathrm{Z}$ resonances, are shown in figures 5.15, 5.16 and 5.17 .

CMX L1 and L2 from $J / \psi, \Upsilon$, and Z in Miniskirts 
Chapter 5. Measurement of Low $p_{T}$ Muon Trigger Efficiencies
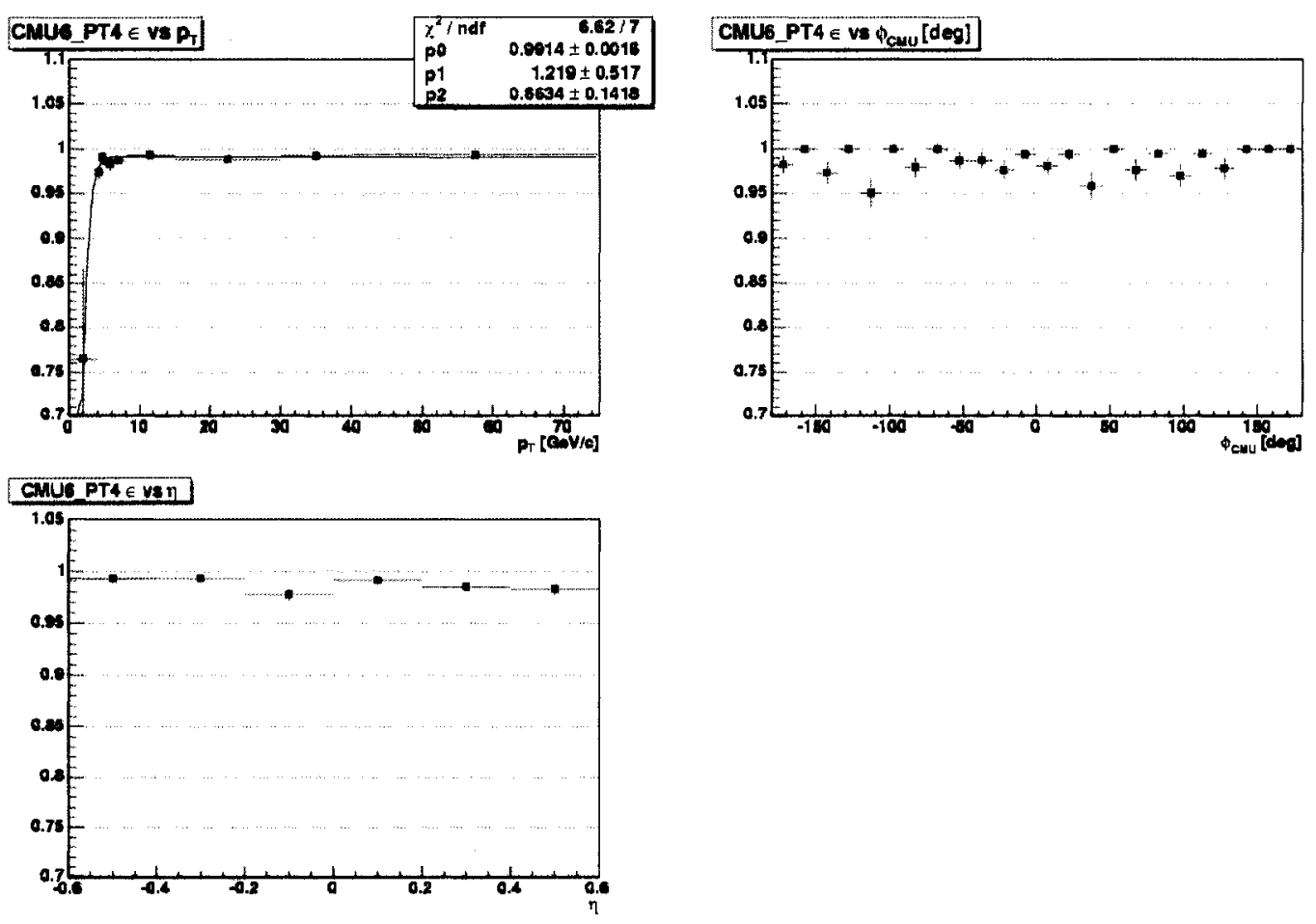

Figure 5.6: $J / \psi$ and Z After SB subtraction and $z M i n A x S L$ cut not applied, single-leg L2 efficiency vs $p_{T}$ (top left), vs $\phi$ (top right), vs $\eta$ (bottom). A fit function shown in blue (top right). The absence of $z \operatorname{Min} A x S L$ does not affect L2 efficiency which is approximately flat as a function of $\phi$ and $\eta$. 
Chapter 5. Measurement of Low $p_{T}$ Muon Trigger Efficiencies
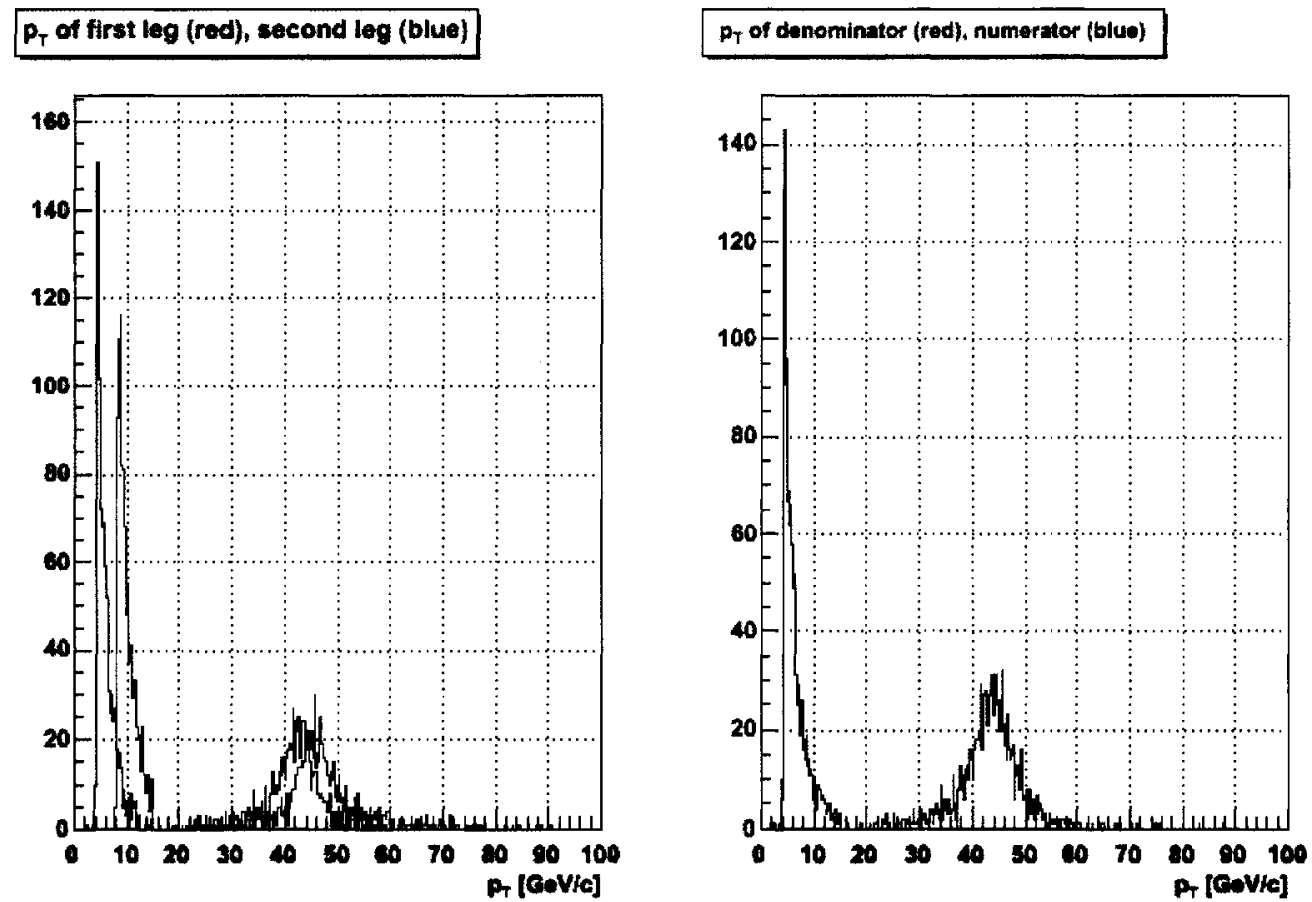

Figure 5.7: $\Upsilon$ and Z Left: $p_{T}$ of trigger leg (red) and other leg (blue) leg. Right: $p_{T}$ of other leg, numerator (blue), denominator (red) 
Chapter 5. Measurement of Low $p_{T}$ Muon Trigger Efficiencies
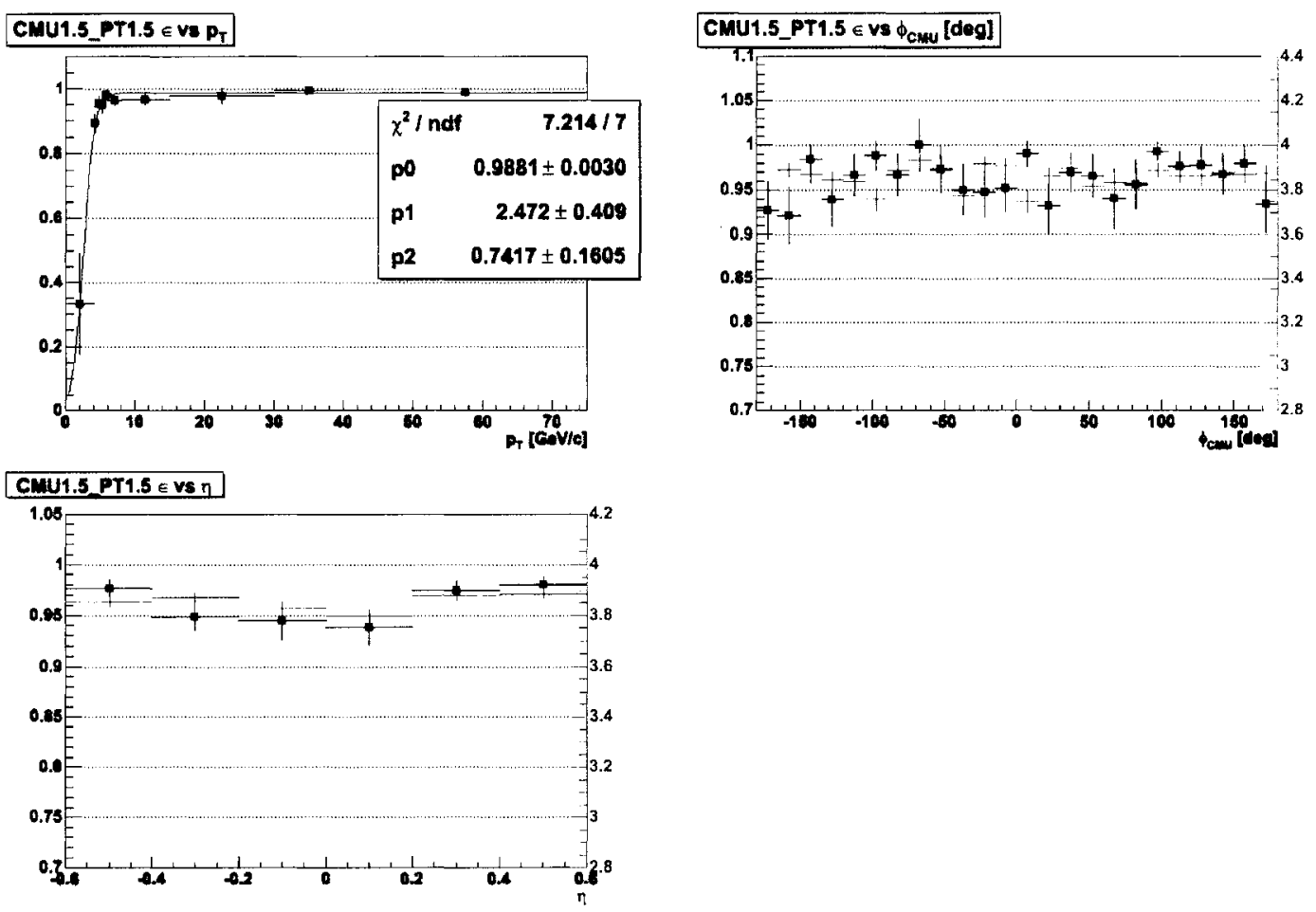

Figure 5.8: $\Upsilon$ and Z After SB subtraction, single-leg L1 efficiency vs $p_{T}$ (top left), vs $\phi$ (top right), vs $\eta$ (bottom). A fit function shown in blue (top right). Number of CMU hits shown in red (top right and bottom left). For $p_{T}>6 \mathrm{GeV} / c$ efficiency becomes flat. Efficiency is approximately flat as a function of $\phi$ and $\eta$. 
Chapter 5. Measurement of Low $p_{T}$ Muon Trigger Efficiencies
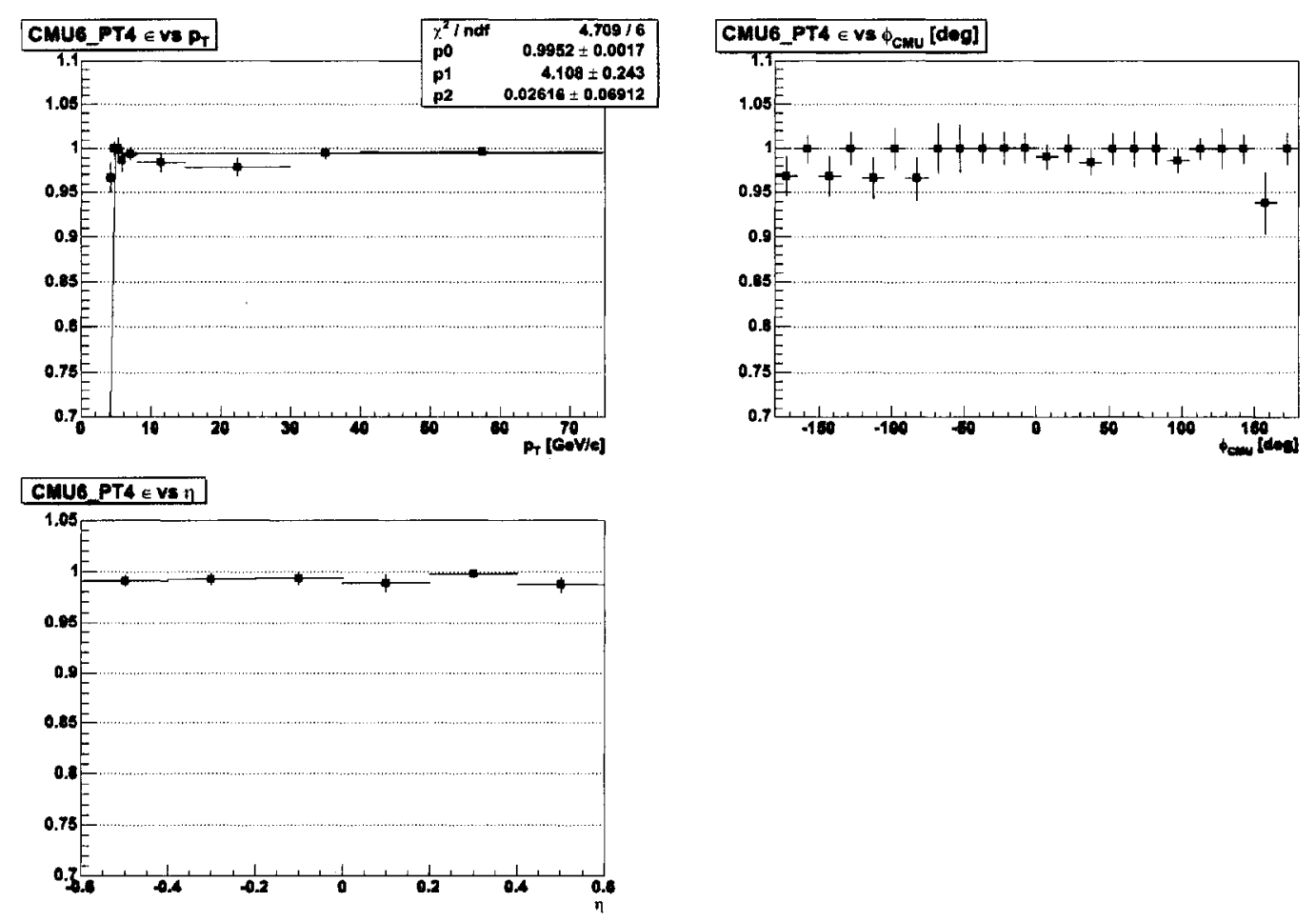

Figure 5.9: $\Upsilon$ and Z After SB subtraction, single-leg L2 efficiency vs $p_{T}$ (top left), vs $\phi$ (top right), vs $\eta$ (bottom). A fit function shown in blue (top right). Number of CMU hits shown in red (top right and bottom left). For $p_{T}>6 \mathrm{GeV} / c$ efficiency becomes flat. Efficiency is approximately flat as a function of $\phi$ and $\eta$. 
Chapter 5. Measurement of Low $p_{T}$ Muon Trigger Efficiencies
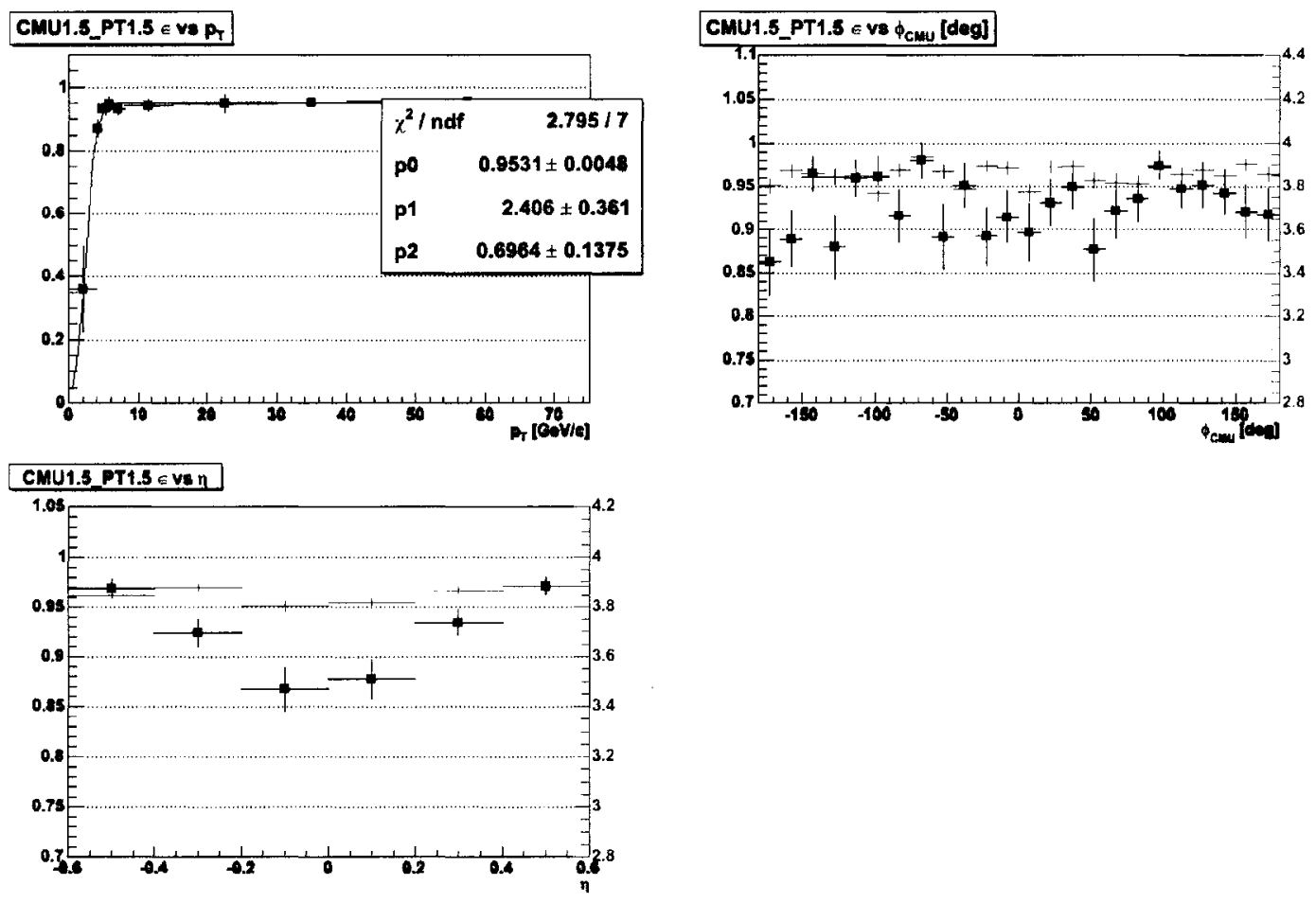

Figure 5.10: $\Upsilon$ and $\mathrm{Z}$ after SB subtraction and $z$ Min $A x S L$ cut not applied, single-leg L1 efficiency vs $p_{T}$ (top left), vs $\phi$ (top right), vs $\eta$ (bottom). A fit function shown in blue (top right). Number of CMU hits shown in red (top right and bottom left). The absence of $z \operatorname{Min} A x S L$ cut lowers the efficiency and introduces a significant $\eta$ dependence. 
Chapter 5. Measurement of Low $p_{T}$ Muon Trigger Efficiencies
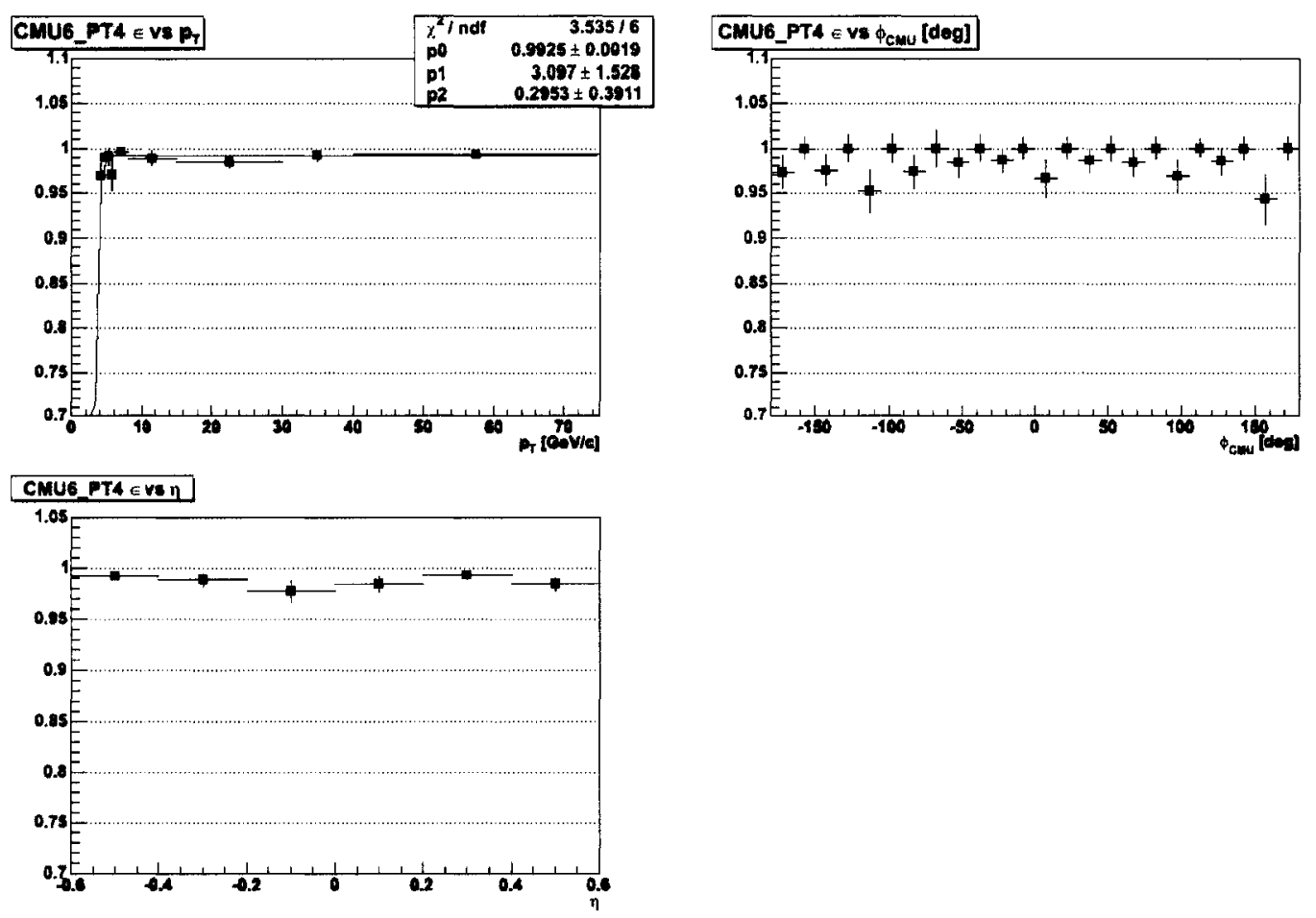

Figure 5.11: $\Upsilon$ and Z After SB subtraction and $z M i n A x S L$ cut not applied, single-leg L2 efficiency vs $p_{T}$ (top left), vs $\phi$ (top right), vs $\eta$ (bottom). A fit function shown in blue (top right). The absence of $z M$ in $A x S L$ does not affect L2 efficiency which is approximately flat as a function of $\phi$ and $\eta$. 
Chapter 5. Measurement of Low $p_{T}$ Muon Trigger Efficiencies

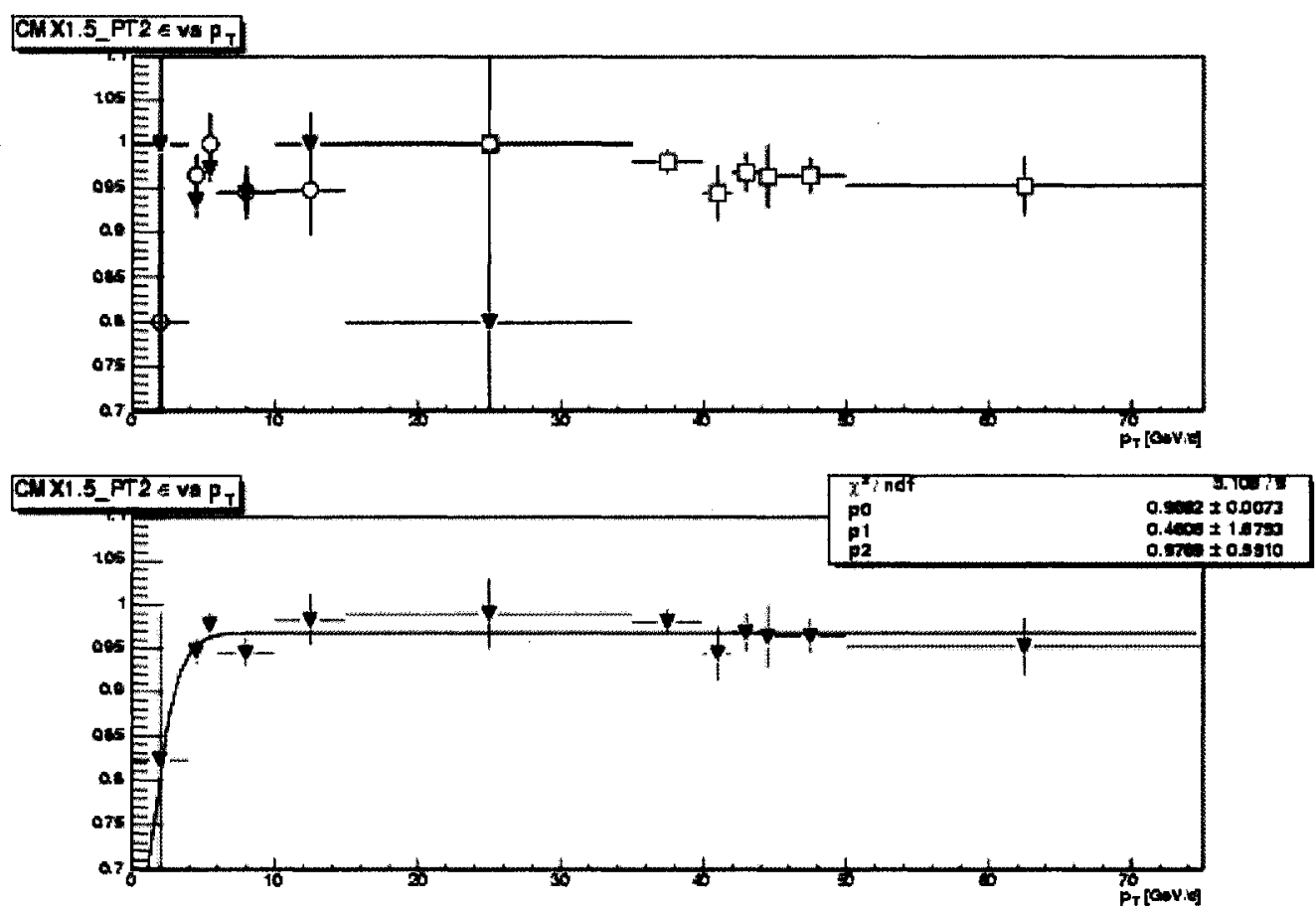

Figure 5.12: L1 CMX efficiency in the east arch. The keystone and the miniskirt are excluded. Top, efficiency as a function of $p_{T}$ from $J / \psi$ in black triangles, from $\Upsilon$ red circles, from $\mathrm{Z}$ in green squares. Bottom, efficiency obtained from $J / \psi, \Upsilon$ and $\mathrm{Z}$ are combined. Although the $p_{T}$ turn on of the efficiency is not very well resolved, the efficiency is constant for $p_{T}>6 \mathrm{GeV} / c$. 

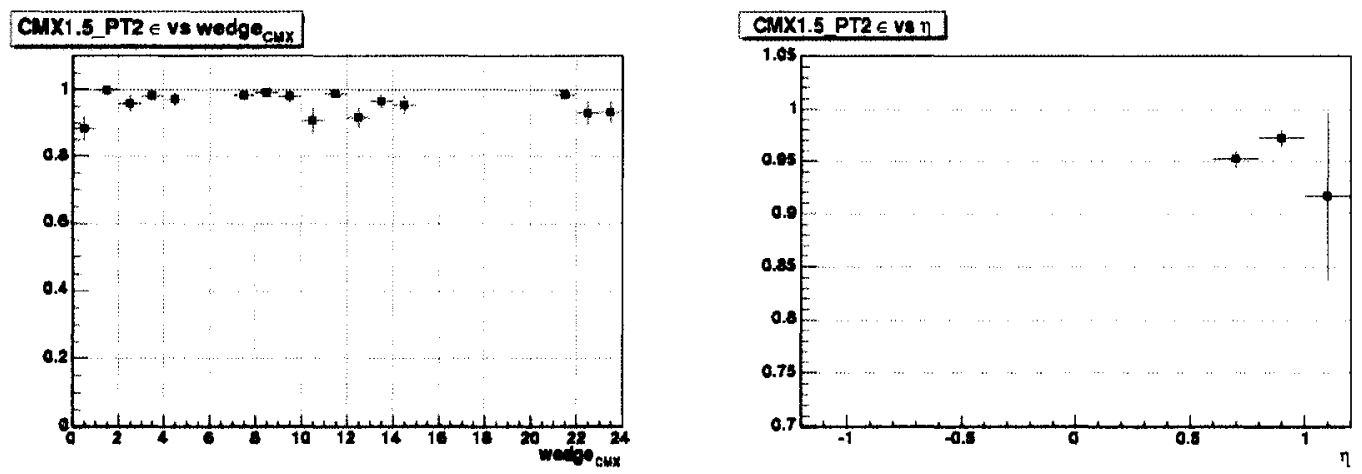

Figure 5.13: L1 CMX efficiency in the arches as a function of CMX wedge number (left) and CMX stub $\eta$. The keystone and the miniskirt are excluded, which is reflected by the missing bins in the plot on the left. In the same plot, the low efficiency bin corresponds to the keystone part of CMX detector. Wedge \#14 West was malfunctioning during the data taking, so we did not consider the muons found in this wedge.
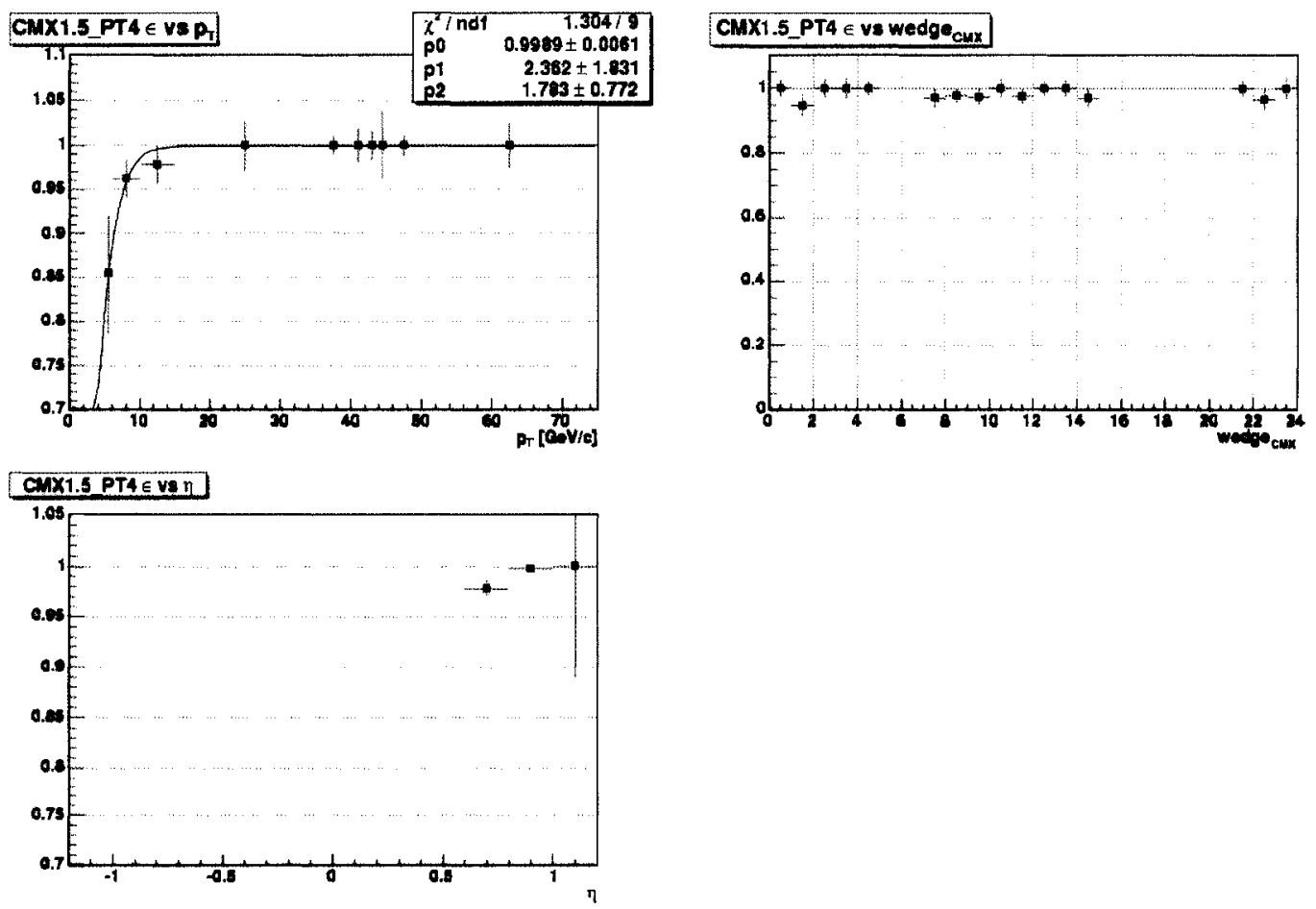

Figure 5.14: L2 CMX efficiency as a function of $p_{T}, \mathrm{CMX}$ wedge number (right) and CMX stub $\eta$ (bottom). The keystone and the miniskirt are excluded. 
Chapter 5. Measurement of Low $p_{T}$ Muon Trigger Efficiencies
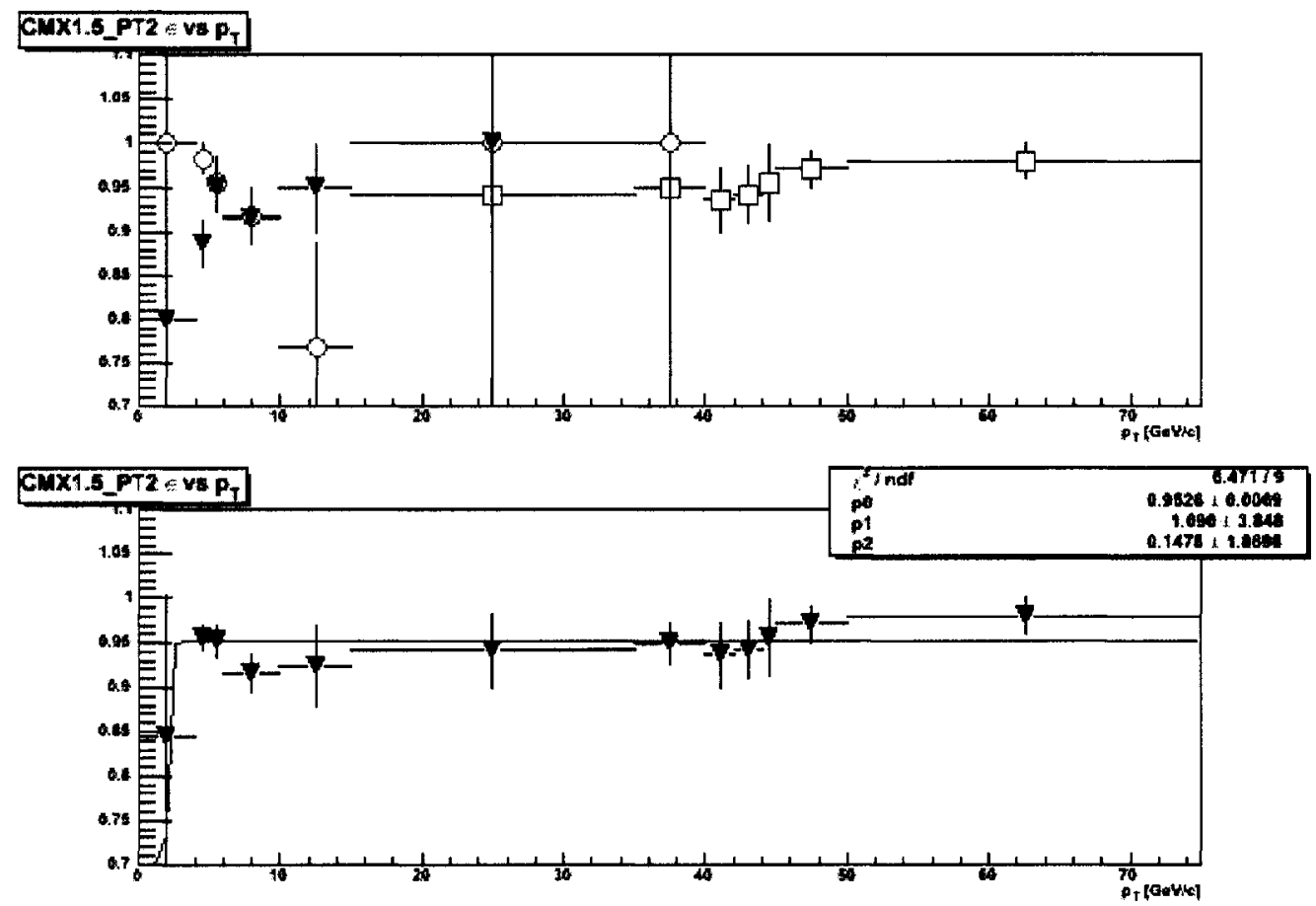

Figure 5.15: L1 CMX efficiency in the west arch. Miniskirts are excluded. Top, efficiency as a function of $p_{T}$ from $J / \psi$ in black triangles, from $\Upsilon$ red circles, from $\mathrm{Z}$ in green squares. Bottom, efficiency obtained from $J / \psi, \Upsilon$ and $\mathrm{Z}$ are combined. Although the $p_{T}$ turn on of the efficiency is not very well resolved, the efficiency is constant for $p_{T}>6 \mathrm{GeV} / c$. 

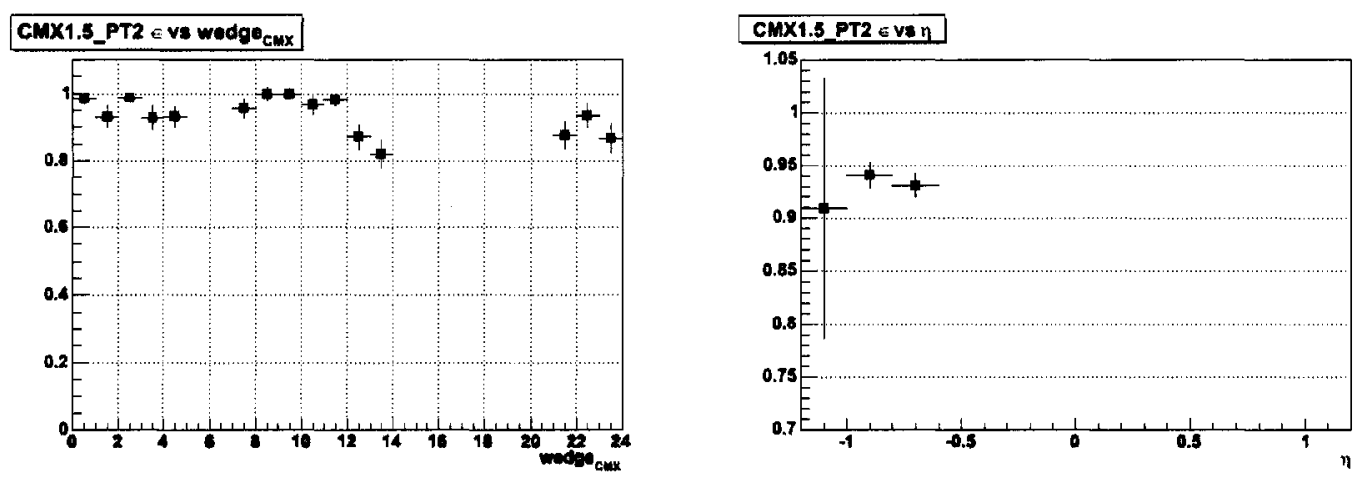

Figure 5.16: L1 CMX efficiency in the arches as a function of CMX wedge number (left) and CMX stub $\eta$. Miniskirts are excluded, which is reflected by the missing bins in the plot on the left. In the same plot, the low efficiency bin corresponds to the keystone part of CMX detector. Wedge \#14 West was malfunctioning during the data taking, so we did not consider the muons found in this wedge.
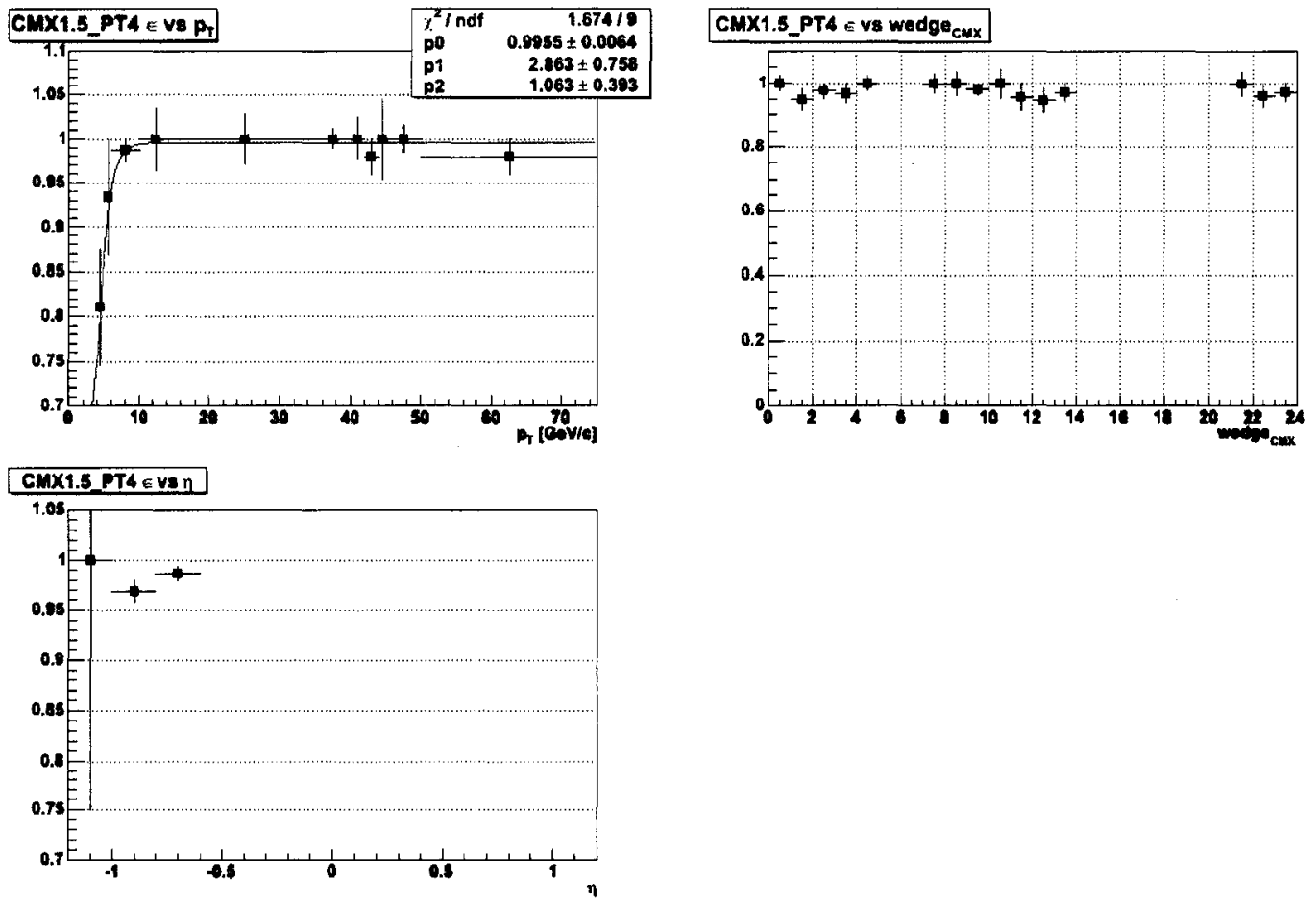

Figure 5.17: L2 CMX efficiency as a function of $p_{T}$, CMX wedge number (right) and CMX stub $\eta$ (bottom). Miniskirts are excluded. 

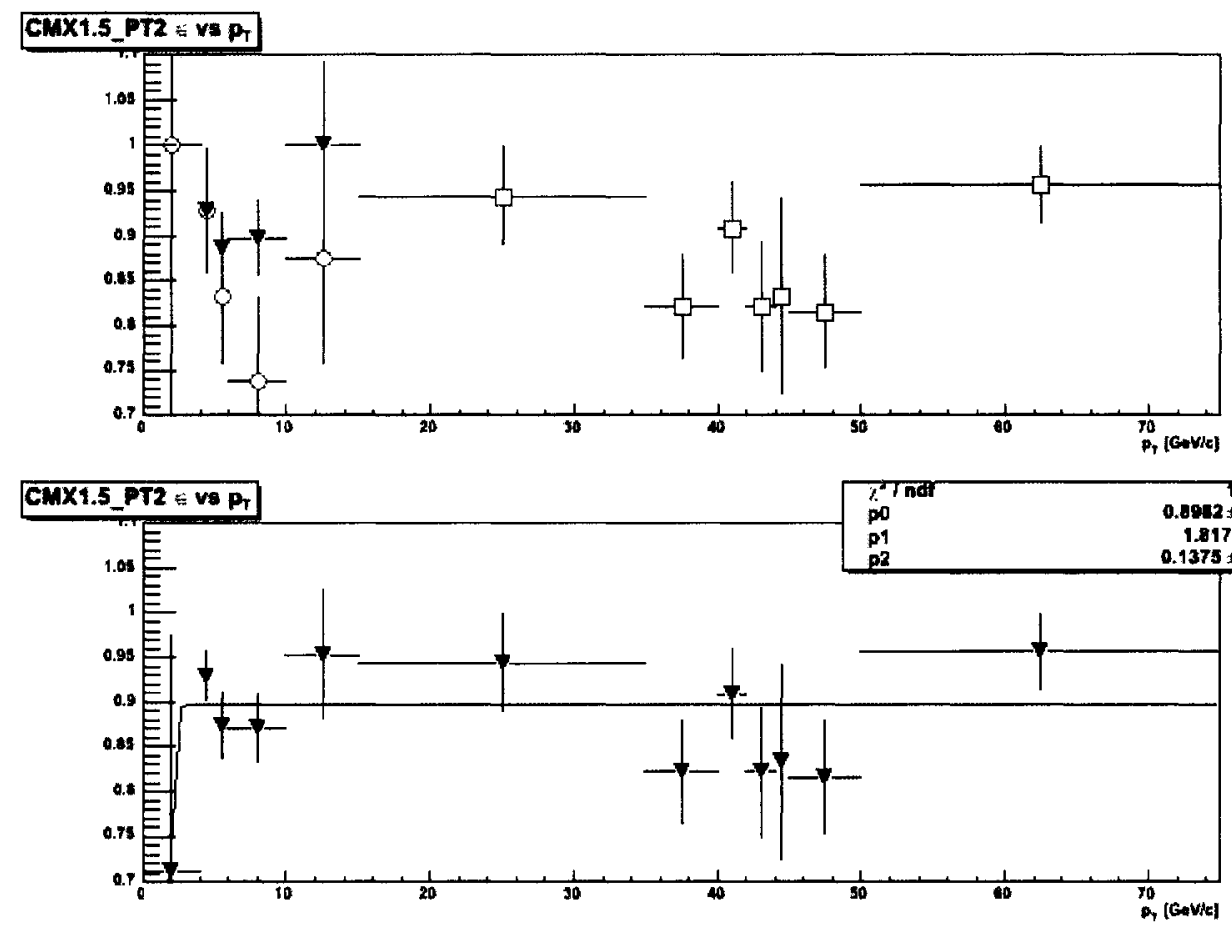

Figure 5.18: L1 CMX efficiency in the miniskirts. Top, efficiency as a function of $p_{T}$ from $J / \psi$ in black triangles, from $\Upsilon$ red circles, from $\mathrm{Z}$ in green squares. Bottom, efficiency obtained from $J / \psi, \Upsilon$ and $\mathrm{Z}$ are combined.
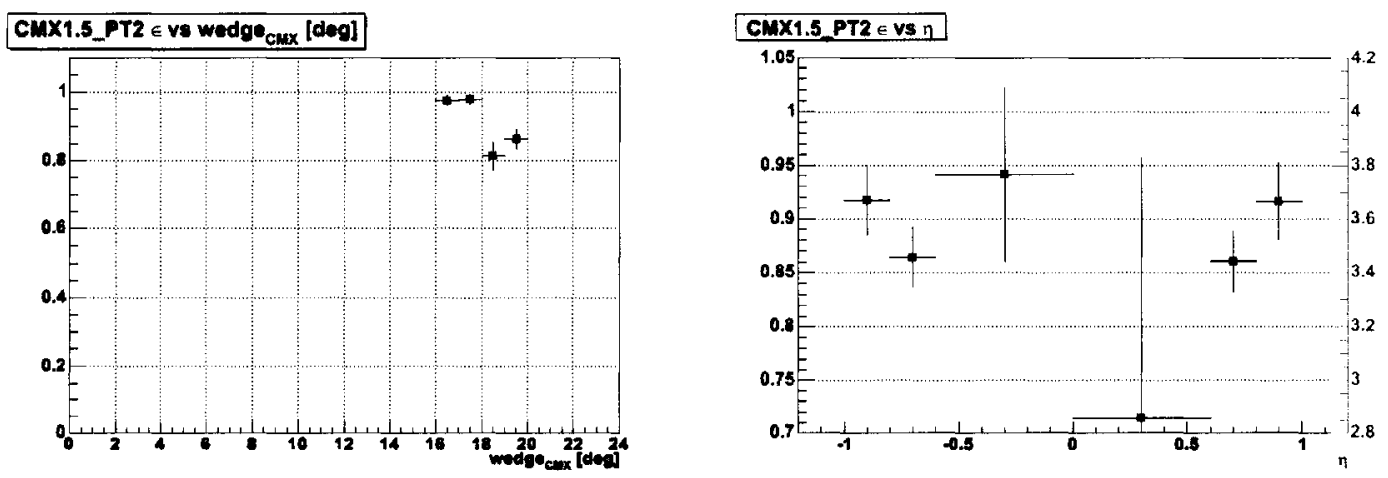

Figure 5.19: L1 CMX efficiency in the miniskirts as a function of CMX wedge number (left) and CMX stub $\eta$ (right). CMX miniskirt wedges \#15 and \#20 were not in function during this data taking so the bins corresponding to these wedges are missing from the plot on the left. 
Chapter 5. Measurement of Low $p_{T}$ Muon Trigger Efficiencies
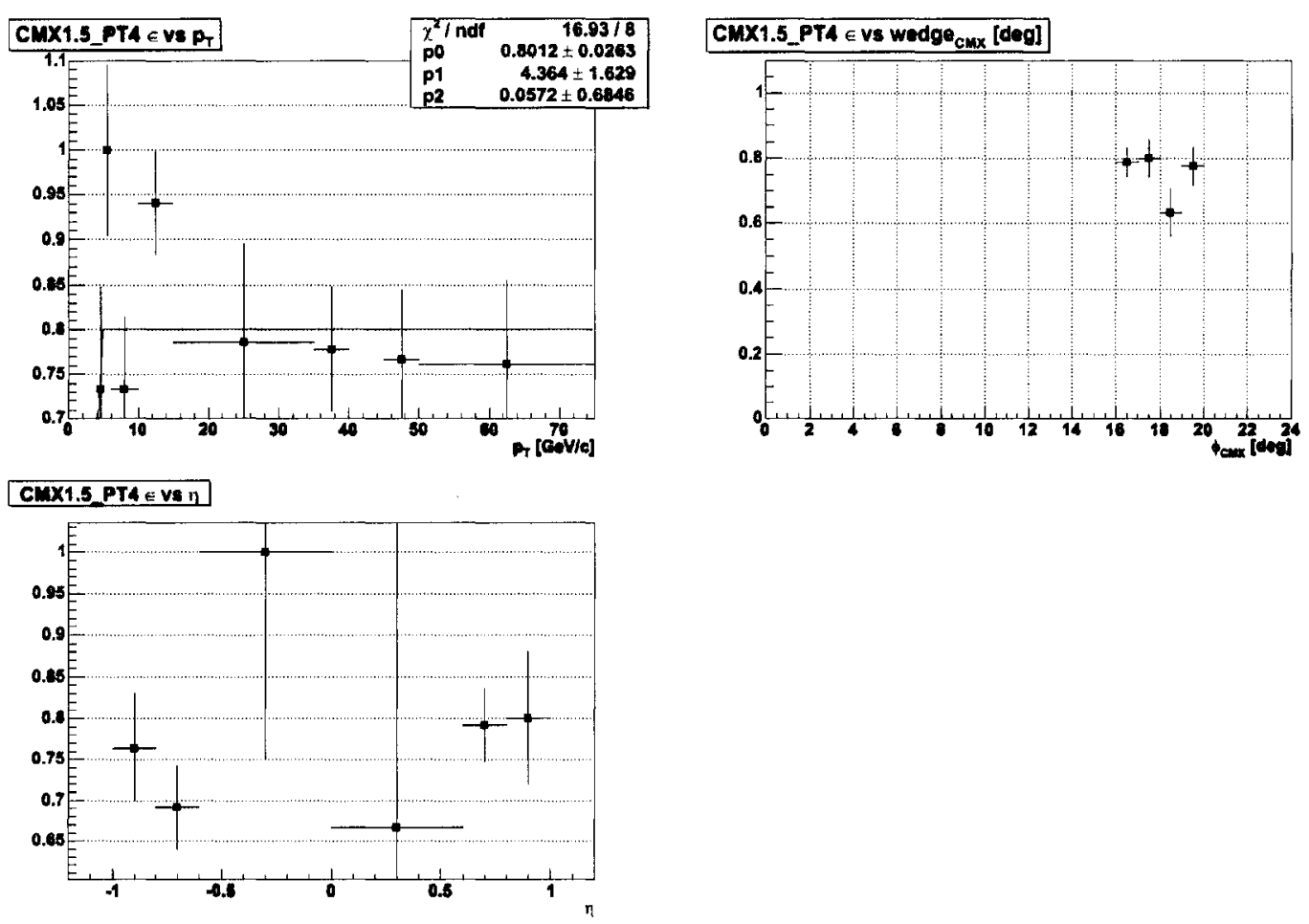

Figure 5.20: L2 CMX efficiency in the miniskirts as a function of $p_{T}$, CMX wedge number (right) and CMX stub $\eta$ (bottom). 
Chapter 5. Measurement of Low $p_{T}$ Muon Trigger Efficiencies

\subsubsection{L3 Single-Leg Trigger Efficiency}

Again, we seek to determine single-leg muon trigger efficiencies. In the following subsections we describe how single-leg L3 CMU and CMX trigger efficiencies were measured.

\section{Dataset}

Calculation of L3 trigger efficiency was done from the same dataset as L1 and L2, SUSY dilepton dataset edil0h. However, we used different triggers and different method from the ones used for the calculation of L1 and L2 efficiencies. For L1 and L2 efficiencies we used dimuon triggers. These triggers had dimuon triggers at L3, which made them unusable for calculation of L3. Instead, we used a single electron trigger ELECTRON_4_LOOSE to select electron-muon events. These electron-muon events provide us with a sample of trigger unbiased muons.

\section{Algorithm and Calculation of Efficiency for L3}

As described in section 2, we define L3 muon efficiency as

$$
\epsilon_{3} \equiv P(L 1, L 2, L 3, \text { offl } \mid \mathrm{L} 1, \mathrm{~L} 2, \mathrm{offl})
$$

To calculate the efficiency of L3 muon trigger we use the offline CdfMuon and the muon part of muon-electron trigger path CEM4_CMU4 or CEM4_CMX4. The electron part of CEM4_CMX4 path are identical to ELECTRON_4_LOOSE path modulo prescale of 250 of ELECTRON_4_LOOSE trigger at L2.

Before the trigger measurement was made, the following requirements were imposed:

- We required L1 muon trigger L1_CMU1.5_PT1.5, L1_CMX1.5_PT2, and L2 trig- 
Chapter 5. Measurement of Low $p_{T}$ Muon Trigger Efficiencies

ger muon L2_CMU6_PT4, L2_CMX1.5_PT4, in the case of CMU and CMX, respectively.

- Offline muon has a $\mathrm{CMU}(\mathrm{X})$ stub.

- We matched the offline CdfMuon to L1 and L2 trigger bits.

- Matched the offline muon to a L3Object within 1 degree in solid angle

- Required hard calorimetry cuts on the offline muon

$$
\begin{aligned}
& -\mathrm{EM}<1 \mathrm{GeV} / c^{2} \\
& -\mathrm{HAD}<2 \mathrm{GeV} / c^{2} \\
& -\mathrm{EM}+\mathrm{HAD}>0.1 \mathrm{GeV} / c^{2}
\end{aligned}
$$

That the requirements above do not bias L3 efficiency calculation can be seen from the requirements of CMU L3 trigger:

L3 Instance: muonCMU4_v1, of class L3MuonFilterModule_v-1
1. $\mathrm{cmuDx}=15$
2. $\operatorname{minPt}=4.0$
3. nMuon $=1$
4. selectCMU $=$ true

All the events that pass the above requirements enter in the denominator of the L3 efficiency calculation. The events that, in addition to the above requirements have CEM4_CMU(X)4 path triggered, enter in the numerator. 
Chapter 5. Measurement of Low $p_{T}$ Muon Trigger Efficiencies

\section{L3 CMU and L3 CMX}

The L3 CMU and L3 CMX trigger efficiency is effectively $100 \%$ for $p_{T}>4 \mathrm{GeV}$. This can be seen in figures 5.21 and 5.22 .
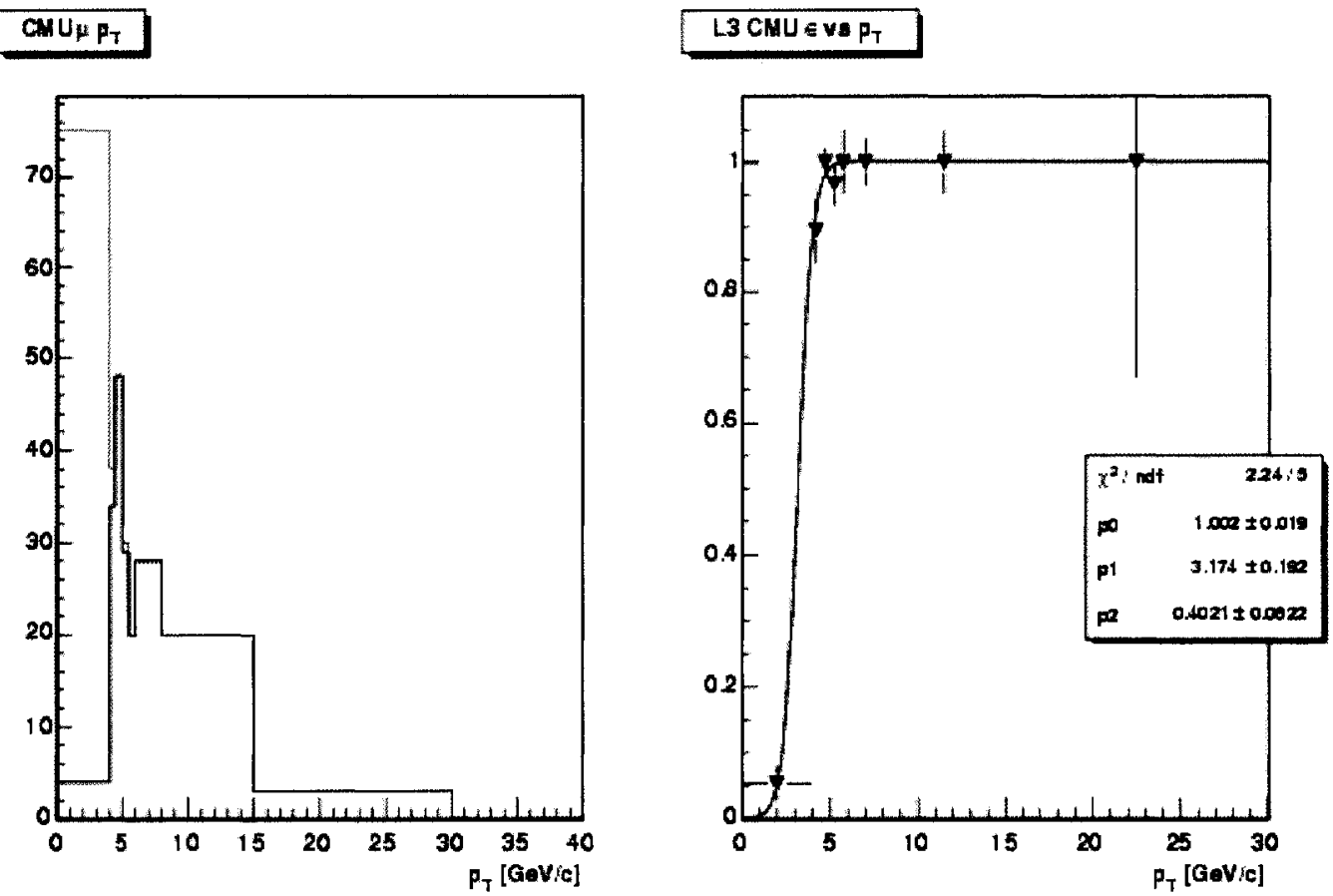

Figure 5.21: L3 CMU. Left: $p_{T}$ distribution of the denominator (red) and the numerator (blue). Right: L3 CMU trigger efficiency. In the first bin, the inefficiency is due to $p_{T}$ cut of $4 \mathrm{GeV}$ at L3, almost entirely. 
Chapter 5. Measurement of Low $p_{T}$ Muon Trigger Efficiencies
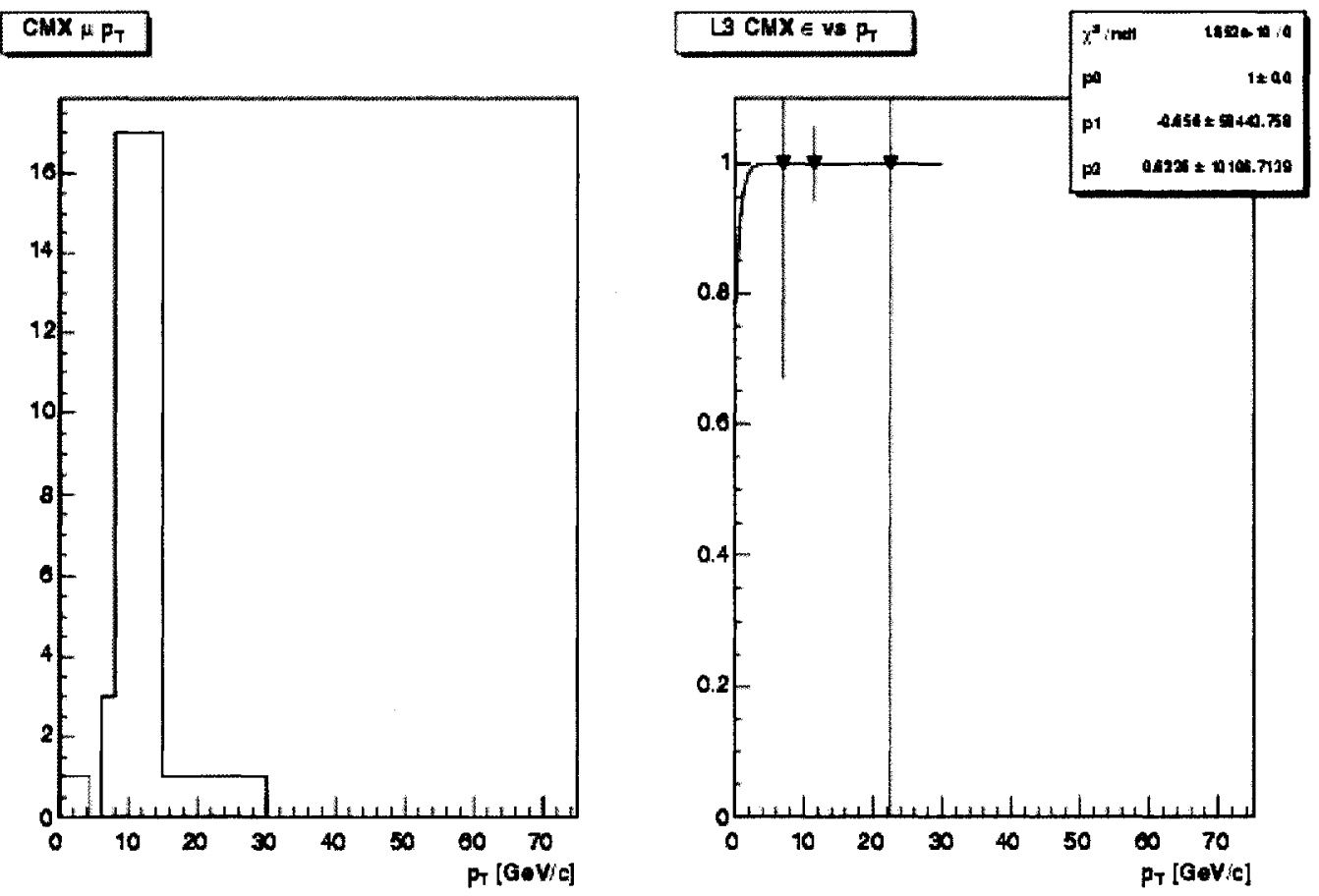

Figure 5.22: L3 CMX. Left: $p_{T}$ distribution of the denominator (red) and the numerator (blue). Right: L3 CMX trigger efficiency. There is much lower statistics in for this measurement to L3 CMU measurement, because CMX of much lower yield of CMX muons. 
Chapter 5. Measurement of Low $p_{T}$ Muon Trigger Efficiencies

\subsubsection{Summary}

We summarize the results of our measurements of efficiency of all three trigger levels in table 5.5 for CMU, table 5.6 for CMX arches and table 5.7 for CMX miniskirts. In these tables we list extracted parameters to the function

$$
f(x)=\frac{p_{0}}{1+\exp \left(p_{1}-\frac{x}{p_{2}}\right)}
$$

, used to fit the plots of trigger efficiencies as a function of $p_{T}$. In these tables we also list "plateau cut-off" value of $p_{T}$ for which the above function reaches plateau, and therefore can be substituted by a constant single value of the parameter $p_{0}$.

\begin{tabular}{|lccccc|}
\hline level & $\mathrm{p} 0$ & $\mathrm{p} 1$ & $\mathrm{p} 2$ & $\chi^{2} / \mathrm{ndf}$ & plateau cutoff[GeV/c] \\
\hline L1 & $0.987 \pm 0.003$ & $1.646 \pm 0.425$ & $0.944 \pm 0.142$ & $17.8 / 7$ & 6 \\
L1 & $0.956 \pm 0.004$ & $1.938 \pm 0.296$ & $0.783 \pm 0.099$ & $16.1 / 7$ & 6 \\
L2 & $0.994 \pm 0.002$ & $0.883 \pm 0.804$ & $0.780 \pm 0.207$ & $6.3 / 7$ & 6 \\
L3 & $1.002 \pm 0.019$ & $3.174 \pm 0.192$ & $0.402 \pm 0.062$ & $2.2 / 5$ & 4 \\
\hline
\end{tabular}

Table 5.5: CMU trigger efficiency fit function parameters by trigger level. L1' and L1 entries list the efficiency fit function parameters for Level 1 with and without $z$ MinAxSL cut imposed, respectively. The effect of this cut is negligible for L2. Parameters extracted from figures 5.3, 5.5, 5.4, 5.21.

\begin{tabular}{|lccccc|}
\hline level & $\mathrm{p} 0$ & $\mathrm{p} 1$ & $\mathrm{p} 2$ & $\chi^{2} / \mathrm{ndf}$ & plateau cut-off \\
\hline (East) L1 & $0.968 \pm 0.007$ & $0.461 \pm 1.875$ & $0.977 \pm 0.551$ & $5.1 / 9$ & 6 \\
(West) L1 & $0.953 \pm 0.007$ & $1.696 \pm 3.848$ & $0.14 r \pm 1.870$ & $6.5 / 9$ & 6 \\
(East) L2 & $0.999 \pm 0.006$ & $2.362 \pm 1.831$ & $1.783 \pm 0.772$ & $1.3 / 9$ & 15 \\
(West) L2 & $0.996 \pm 0.006$ & $2.863 \pm 0.758$ & $1.063 \pm 0.393$ & $1.7 / 9$ & 15 \\
L3 & $1.000 \pm 0.000$ & $-0.656 \pm 58443$ & $0.626 \pm 10106$ & $1.8 / 2$ & 4 \\
\hline
\end{tabular}

Table 5.6: CMX trigger efficiency fit function parameters by trigger level for the East and West CMX Arch. Result for L3 is obtained from muons from both East and West CMX Arches. Parameters extracted from figures 5.12, 5.15, 5.14, 5.17 and 5.22. 
Chapter 5. Measurement of Low $p_{T}$ Muon Trigger Efficiencies

\begin{tabular}{|lccccc|}
\hline level & $\mathrm{p} 0$ & $\mathrm{p} 1$ & $\mathrm{p} 2$ & $\chi^{2} / \mathrm{ndf}$ & plateau cut-off $[\mathrm{GeV} / c]$ \\
\hline L1 & $0.898 \pm 0.014$ & $1,817 \pm 4.448$ & $0.137 \pm 3.333$ & $10.4 / 9$ & 4 \\
L2 & $0.801 \pm 0.026$ & $4.364 \pm 1.629$ & $0.057 \pm 0.685$ & $16.9 / 8$ & 15 \\
L3 & $1.000 \pm 0.000$ & $-0.656 \pm 58443$ & $0.626 \pm 10106$ & $1.8 / 2$ & 4 \\
\hline
\end{tabular}

Table 5.7: Miniskirt CMX trigger efficiency fit function parameters by trigger level. Parameters extracted from figures 5.18, 5.20 and 5.22. 


\section{Chapter 6}

\section{Muon Isolation Efficiencies}

\subsection{Motivation}

We are interested in calculating the efficiency of a muon to be isolated. This efficiency is necessary and useful for many searches. One example is tri-lepton search for chargino-neutralino SUSY production, where the three leptons are isolated. One ultimately might be interested in the efficiency of a muon to pass both isolation and ID requirements. If the isolation efficiency and the ID efficiency of isolated muons are measured, and with Bayes's theorem in hand, one can calculate the efficiency of a muon to pass both isolation and ID requirements in the following manner ${ }^{1}$ :

$$
\epsilon(I D \& I S O)=\epsilon(I D \mid I S O) \epsilon(I S O)
$$

Finally, we derive a scale factor to correct the muon isolation efficiency of the MC.

\footnotetext{
${ }^{1}$ The muon ID efficiencies are measured in CDF not 7210.
} 
Chapter 6. Muon Isolation Efficiencies

\subsection{Algorithm and Samples}

To study the muon isolation efficiency one needs a sample of "good" muons. Good here means that one is confident that these particles are from a well-understood source of prompt, isolated muons, and are not either fakes, cosmics or from sequential decays of heavy-flavor particles. Since we are interested in a large range of muon momenta, we select muons that are likely to be from the Drell-Yan process which produces two leptons of the same flavor and un-like sign charge (US). We do not make the US requirement until the very end. We use the like sign (LS) pairs to model the heavy flavor background distribution. After we subtract the backgrounds, we define the isolation efficiency as the number of events with both legs isolated divided by the number of events with one leg isolated.

$$
\epsilon=\frac{N_{2 i s o}}{N_{1 i s o}}
$$

We define a muon to be isolated if

- ConeR4_Et/ $\mathrm{p}_{T}<0.1$, for $\mathrm{p}_{T}>20 G e V$

- ConeR4_Et $<2 \mathrm{GeV}$, otherwise

where ConeR4_Et is defined as the transverse energy, $\mathrm{E}_{T}$ deposited in the calorimeter towers in the cone of solid angle 0.4 in $\eta$ - $\phi$ space around the muon's tower, excluding the muon's tower.

\subsubsection{DY Selection}

For MC we used Pythia Drell-Yan sample generated, simulated in cdfosft2 version 5.3.3. For data we used SUSY di-lepton data set edil0d, produced in 5.3.1. On both data and $\mathrm{MC}$ were stored in stntuples. We used the production version of Stntuple 
Chapter 6. Muon Isolation Efficiencies

dev_242 package executable, version 5.3.3, so that both data and MC were analyzed in the same production version (cdfsoft2 5.3.3)

Datasets:

- MC - Pythia (Stntuple_prod 5.3.3)

- DATA - edil0d (Stntuple_prod 5.3.3)

To obtain our sample of DY-enriched muons we made the following requirements.

- At least 2 CdfMuon in the event. Figure 6.1

- The two muons' COT tracks be no less then $4 \mathrm{~cm}$ in z0. $(\mu$ 's $|\Delta z 0|<4)$ Figure $6.1(\mathrm{~b})$

- COT exit radius $>140 \mathrm{~cm}$ Figure 6.1 (c).

- Identify the primary vertex closest to the two muons in Z.

- The primary vertex is within $60 \mathrm{~cm}$ from the collision point $(|Z|<60 . \mathrm{cm})$ Figure $6.1(\mathrm{~d})$

- Far from resonances $\left(\mathrm{m}_{\mu \mu}>12\right)$ Figure 6.2 (top)

- Opening angle $\mathrm{b} / \mathrm{w}$ the two COT tracks greater then $150^{\circ}$, i.e. tracks are back to back $(\Delta \phi)>2.5 \mathrm{rad}\left(150^{\circ}\right)$ Figure 6.2 (bottom)

- One $\mu$ leg pass ID cuts (no D0 requirement)

- Testing $\mu$ leg pass track quality cut (number of COT hits $>60$, and no D0 requirement) 
Chapter 6. Muon Isolation Efficiencies
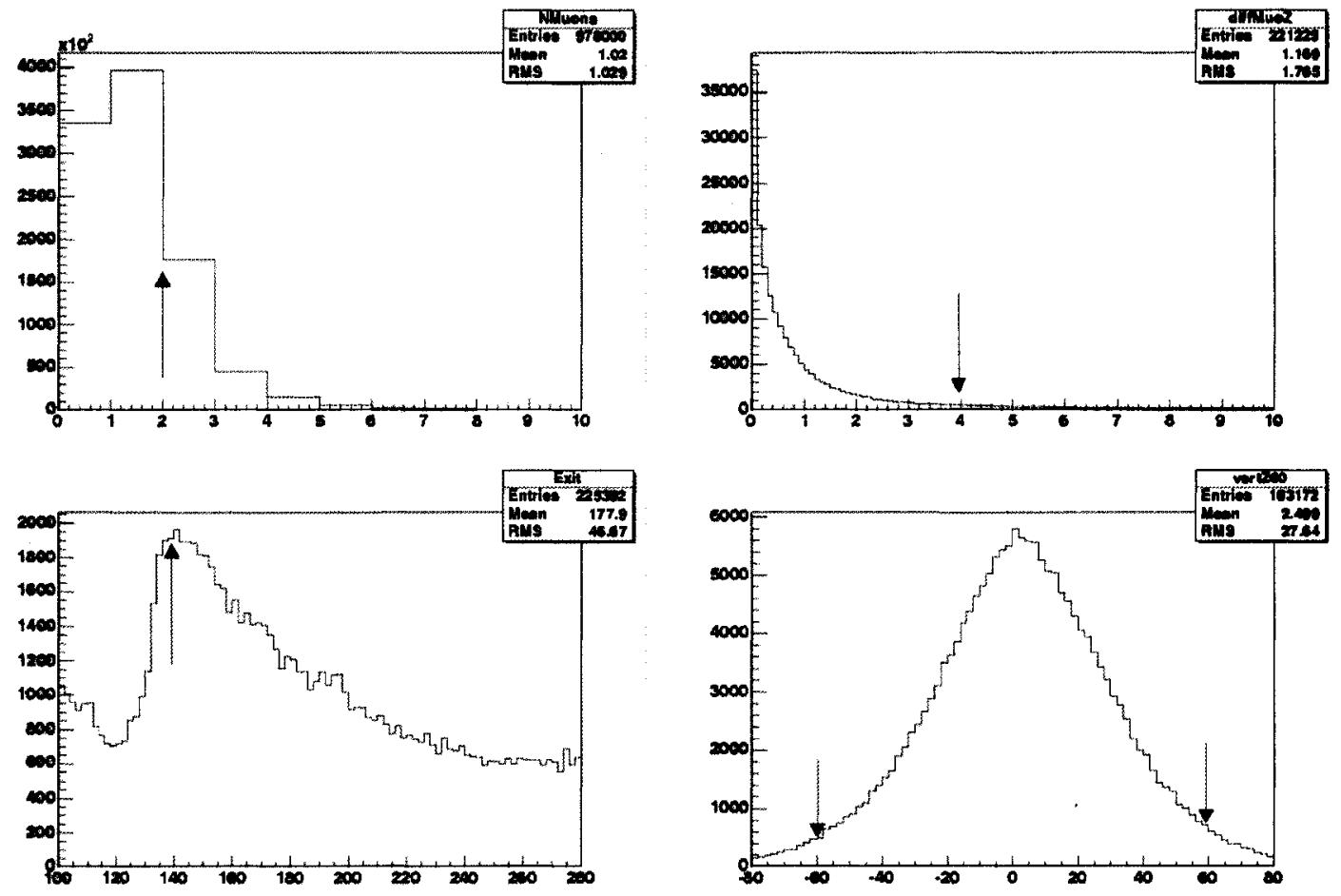

Figure 6.1: Selection of events shown for a part of edil0d DATA: (a) number of CdfMuons in the event, require 2 at least, (b) Difference between the $z_{0}$ coordinates of the muons' COT tracks in $\mathrm{cm}$, (c) COT exit radius in $\mathrm{cm}$, require $\rho_{\text {exit }}>140 \mathrm{~cm}$ (d) $z$ coordinate of selected primary vertex in $\mathrm{cm}$, require $\left|z 0_{\text {prim }}\right|<60 \mathrm{~cm}$

\subsubsection{ID Variables}

In the selection process, we impose ID cuts (all except D0 cut) on the first randomly selected leg. The ID cuts are shown in Table 6.1. We do not apply the calorimeter ID cuts on the $2^{\text {nd }}$ leg, in order to not bias the isolation efficiency. 
Chapter 6. Muon Isolation Efficiencies

Selection of DY mu-mu events

Mon Dec 20 18:12:34 2004
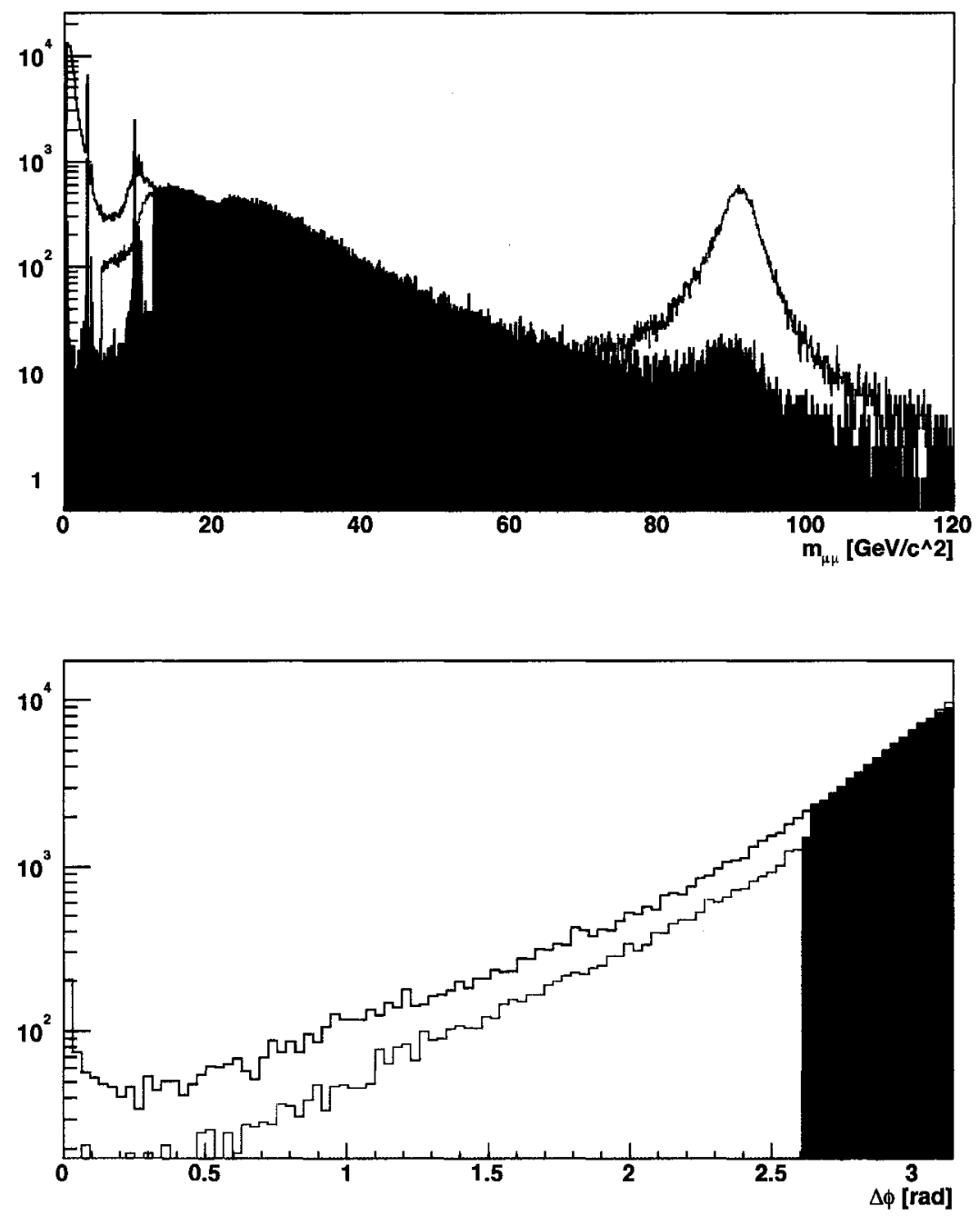

Figure 6.2: Selection of events $\mu \mu$ pairs for all DATA (blue) and MC (red). Top: invariant mass $\left(\mathrm{m}_{\mu \mu}\right)$ of muon pair. For reference we show $\mathrm{m}_{\mu \mu}$ of DATA when both legs isolated (green). Selected events have $\mathrm{m}_{\mu \mu}>12 \mathrm{GeV}$ to assure absence of $\mathrm{J} / \Psi$ and $\Upsilon$ resonances. Bottom: difference of two legs' COT tracks angles, i.e. opening angle $\Delta \phi$. Selected events shown in filled blue $\Delta \phi>150^{\circ}$ 
Chapter 6. Muon Isolation Efficiencies

\begin{tabular}{ll} 
cut & value \\
\hline $\operatorname{Pt}(\mathrm{bc})$ & $>4$ \\
$\mathrm{EM}$ & $<4$ \\
$\mathrm{HAD}$ & $<1$ \\
$\mathrm{EM}+\mathrm{HAD}$ & $>0.1$ \\
$\mathrm{CMU} \Delta x$ & $<3$ or $\chi^{2}<9$ \\
$\mathrm{CMP} \Delta x$ & $<5$ or $\chi^{2}<9$ \\
$\operatorname{CMX} \Delta x$ & $<6$ or $\chi^{2}<9$
\end{tabular}

Table 6.1: ID cuts for muons: CMU-muon has a CMU stub, CMUP-muon has both CMU and CMP stubs, and a CMX-muon a CMX stub

\subsubsection{Backgrounds}

In Tevatron, production of HF pairs $b \bar{b}$ and $c \bar{c}$ is the dominant background to DY. HF quark-antiquark pair can further decay semileptonically into a pair of lepotons of either LS or US charges. We will use the LS data to model the HF background, but we must determine the correct normalization. HF background does not come from the primary interaction, therefore it should be independent of the impact parameter $d_{0}$. On the contrary, the signal DY comes from the primary interaction and the virtual $\gamma$ or Z, and its cross section should depend on $d_{0}$. We exploit this fact in the following manner: - we do not impose any $d_{0}$ cut on LS pairs and use $\mathrm{p}_{T}$ and $\mathrm{m}_{\mu \mu}$ distributions to model the background. (Shown on the right of Figures 6.5 and 6.4. - we impose a tight $d_{0}$ cut $\left(\left|d_{0}\right|<0.005 \mathrm{~cm}\right)$ on US pairs and use $\mathrm{p}_{T}$ and $\mathrm{m}_{\mu \mu}$ distributions to obtain DY signal. (Shown on the left of Figures 6.5 and 6.4. - we estimate the HF background contribution in the US sample using the LS pair sample, which is discussed in subsection 6.2.4. 
Chapter 6. Muon Isolation Efficiencies
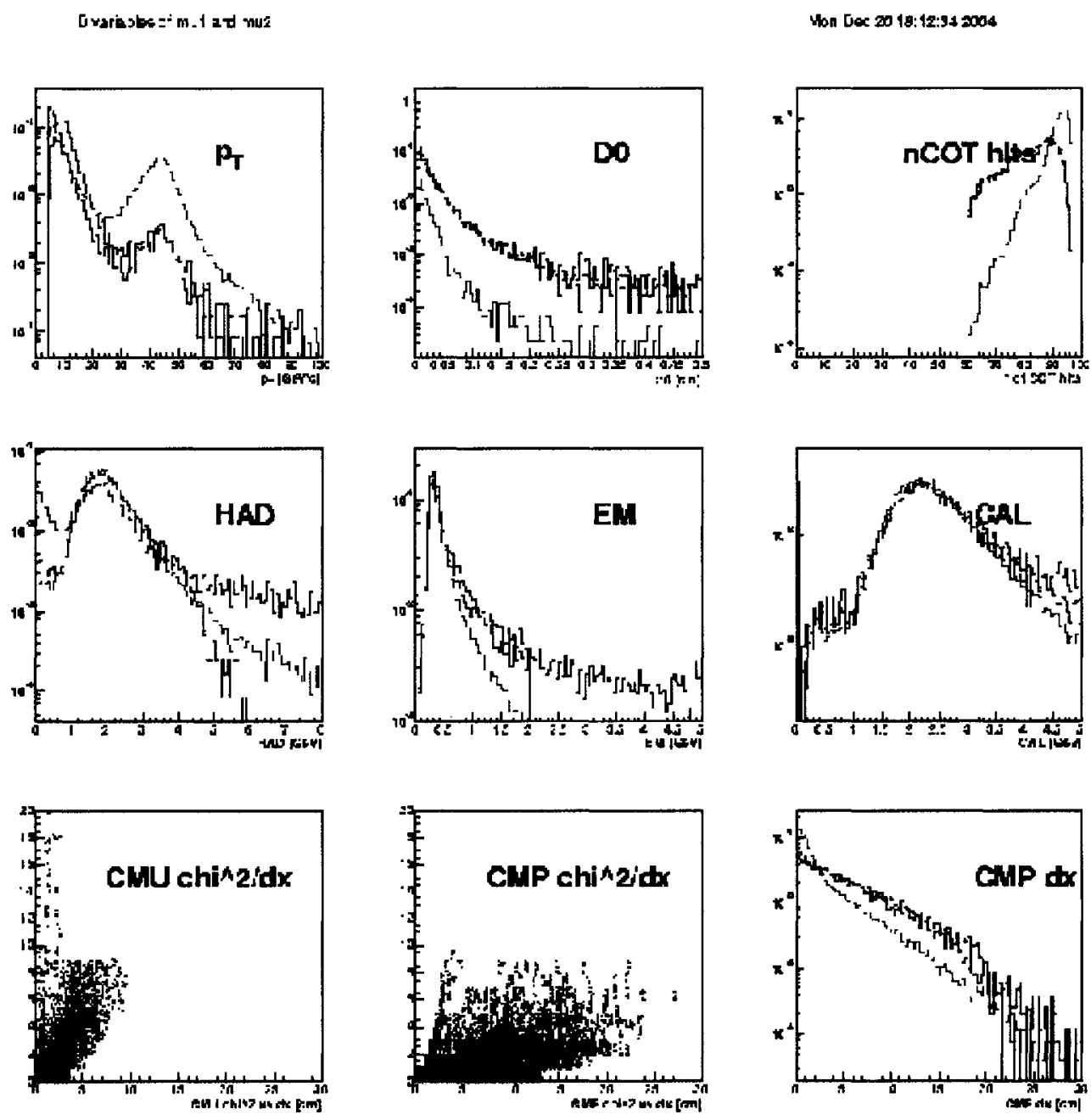

Figure 6.3: $\mu$ ID variables. One leg (black), testing leg (blue), MC testing leg (red). All ID cuts, except for D0 cut, applied to the first leg. Only the matching cuts (cmup $\Delta x$ and $\chi_{c m u p}^{2}$ ) and track quality cut (number of COT hits) applied on the second leg, i.e. testing leg. 
Chapter 6. Muon Isolation Efficiencies

PT Distribution
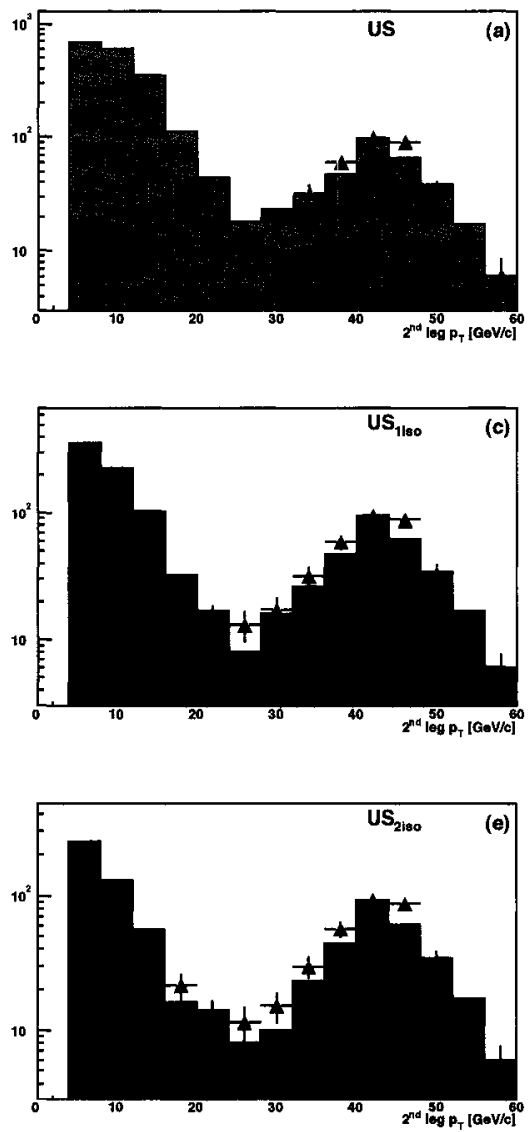

$100012 \cdot 352004$
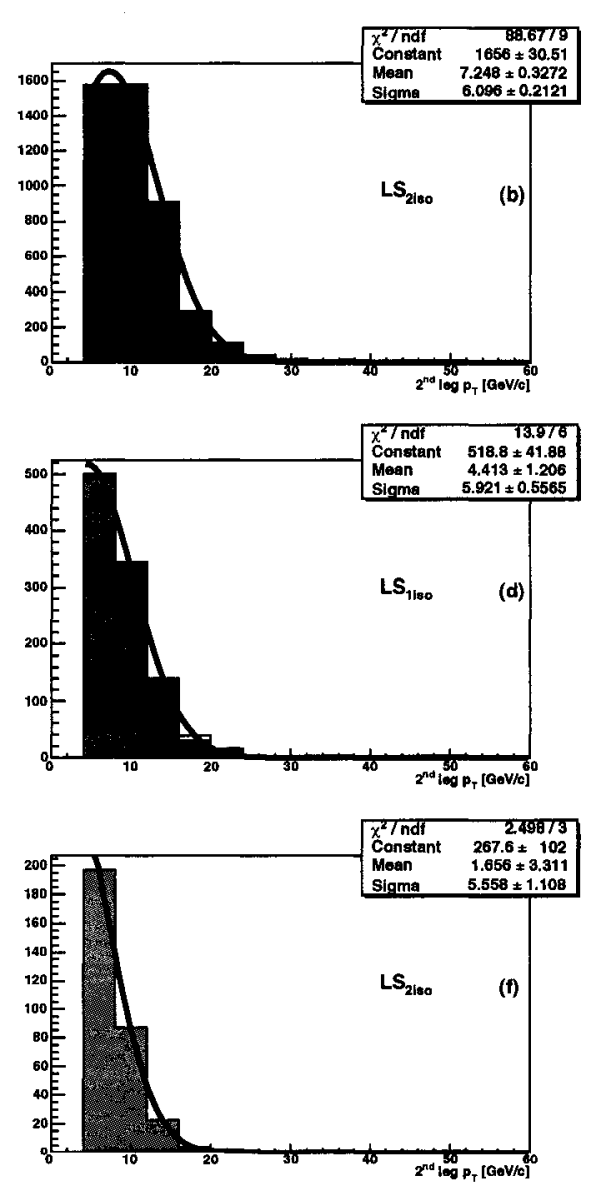

Figure 6.4: $2^{\text {nd }} \operatorname{leg} p_{T}$ : for unlike sign (US) pairs on the left and like sign (LS) pairs on the right. For reference, MC DY normalized to the $\mathrm{Z}$ peak overlaid in red on the left. Negligible number of LS MC events not shown on the right. (a) US pairs (b) LS pairs, (c) US pairs 1 leg isolated, (d) US pairs 1 leg isolated, (e) US pairs both legs isolated, (f) LS pairs both legs isolated. On the right, for LS pairs, a fit with a Gaussian function is shown in black line. 
Chapter 6. Muon Isolation Efficiencies

Invariant Mass Distributions
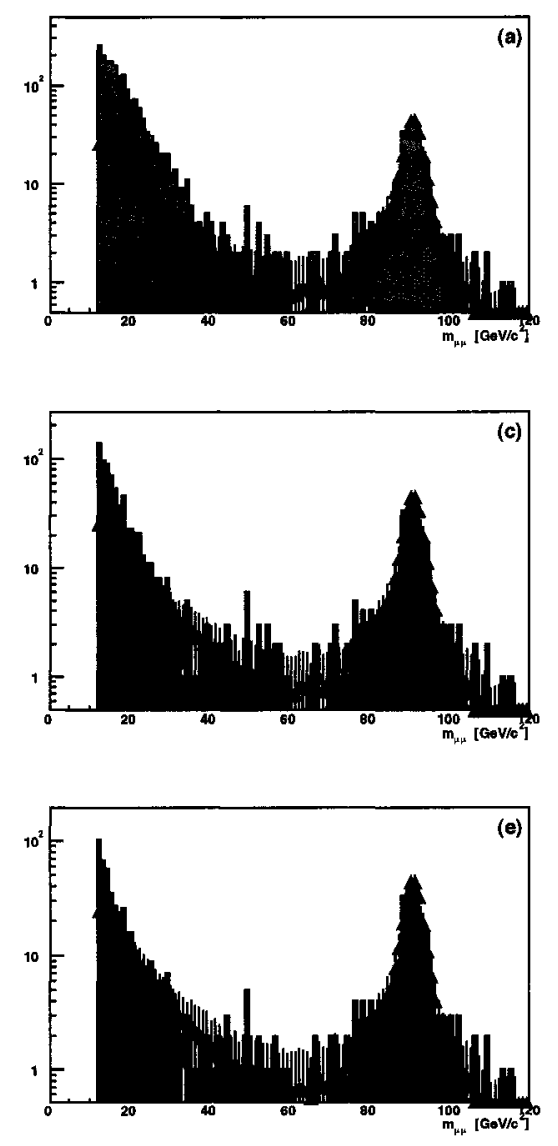

Mon Dec 20 18:12:35 2004
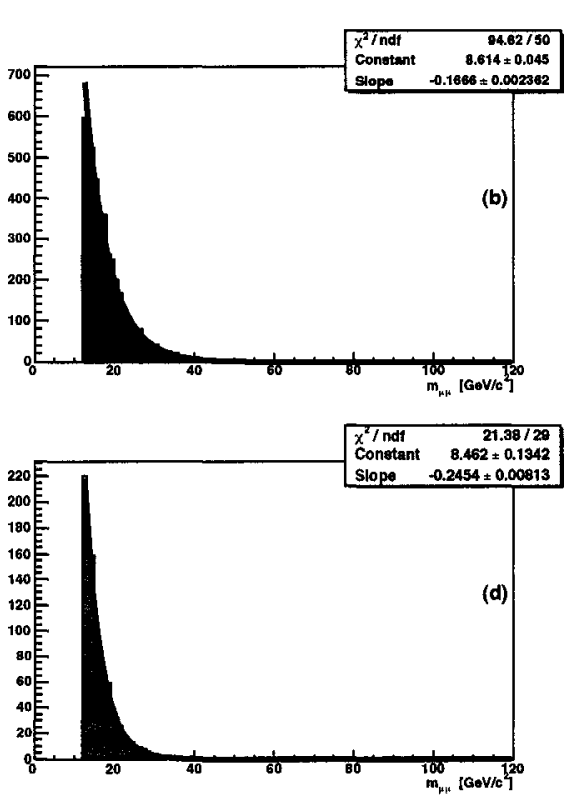

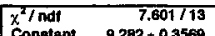

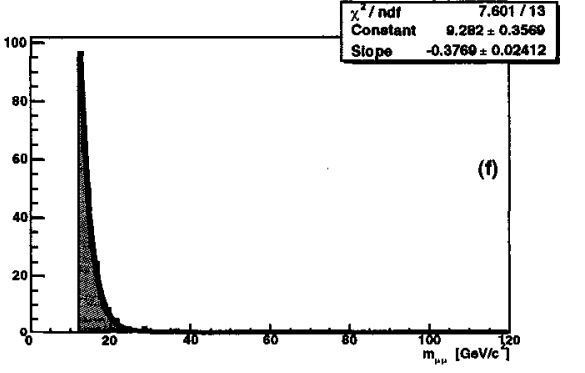

Figure 6.5: $m_{\mu \mu}: \operatorname{leg} p_{T}$ : for unlike sign (US) pairs on the left and like sign (LS) pairs on the right. For reference, MC DY normalized to the $\mathrm{Z}$ peak, overlaid in red on the left. Negligible number of LS MC events not shown on the right. (a) US pairs (b) LS pairs, (c) US pairs 1 leg isolated, (d) US pairs 1 leg isolated, (e) US pairs both legs isolated, (f) LS pairs both legs isolated. On the right, for LS pairs, a fit with a exponential function is shown in black line. 

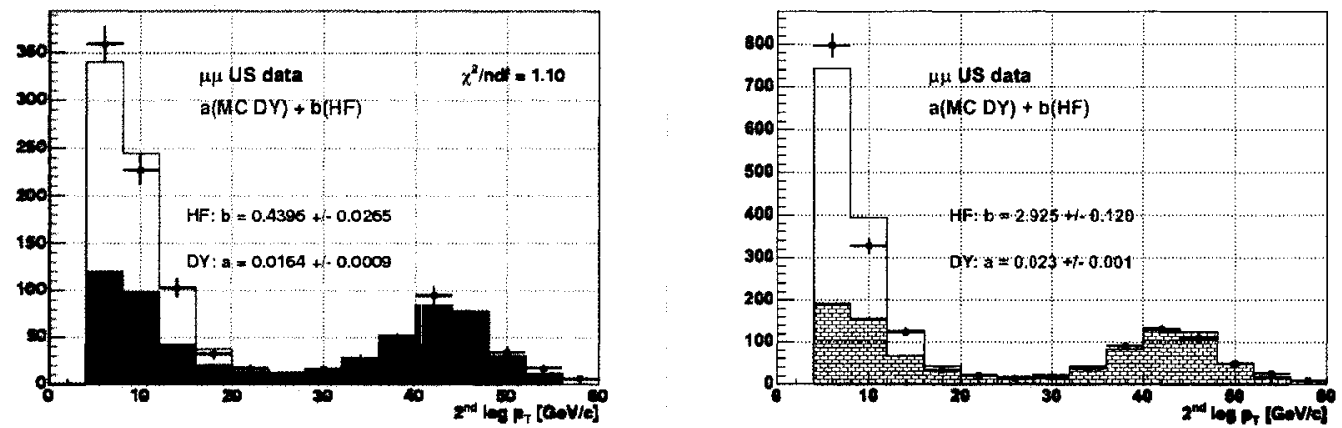

Figure 6.6: Bin-Likelihood fit (a) fit to $\mathrm{p}_{T}$ of the $2^{\text {nd }}$ leg for events where one leg isolated (b) fit to $\mathrm{p}_{T}$ of the $2^{\text {nd }}$ leg for events where both legs isolated
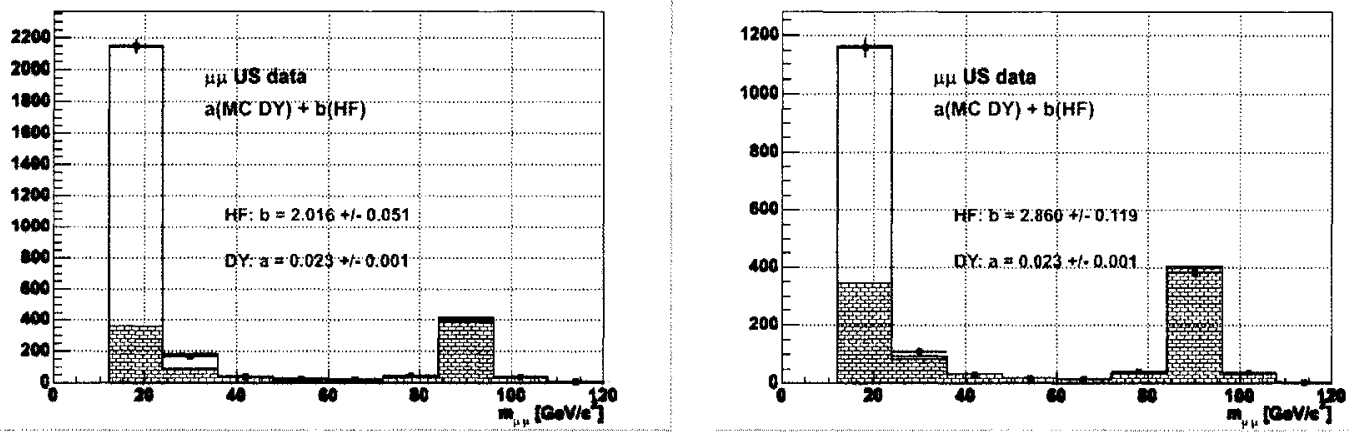

Figure 6.7: Bin-Likelihood fit (a) fit to $\mathrm{m}_{\mu \mu}$ for events where one leg isolated (b) fit to $\mathrm{m}_{\mu \mu}$ for events where both legs isolated

\subsubsection{Bin-Likelihood}

The data US sample referred to in the subsection "Backgrounds" is composed predominantly of DY and HF events. We fit MC DY US and data LS to data US for both invariant mass and $\mathrm{p}_{T}$ of the $2^{\text {nd }} \mathrm{leg}$. We do the fit for both "one-leg isolated" and "both-leg isolated" samples. The results of fitting for $\mathrm{m}_{\mu \mu}$ are shown in Figures 6.6 and 6.7, and the factors of HF LS distributions are given in Tables 6.2 and 6.3. 
Chapter 6. Muon Isolation Efficiencies

\begin{tabular}{l|cc} 
sample & HF LS & MC DY \\
\hline $1_{\text {iso }}$ & $0.4396 \pm 0.0265$ & $0.0164 \pm 0.0009$ \\
$2_{\text {iso }}$ & $0.6502 \pm 0.0685$ & $0.0159 \pm 0.0009$
\end{tabular}

Table 6.2: Bin-Likelihood factors for $\mathrm{p}_{T}$ HF LS and MC DY distributions, also shown in middle row ( $1_{\text {iso }}$ case) and bottom row ( $2_{\text {iso }}$ case) of Figure 6.5 where HF LS is on right (green histogram) and MC DY is on rihgt (red markers). The sum of apropriately weighted HF LS and MC DY histogram approximate DATA US histgram on right (blue histogram)

\begin{tabular}{l|cc} 
sample & HF LS & MC DY \\
\hline $1_{\text {iso }}$ & $0.4443 \pm 0.0258$ & $0.0162 \pm 0.0010$ \\
$2_{\text {iso }}$ & $0.6502 \pm 0.0621$ & $0.0159 \pm 0.0010$
\end{tabular}

Table 6.3: Bin-Likelihood factors for $\mathrm{m}_{\mu \mu}$ HF LS and MC DY distributions, also shown in middle row ( $1_{i s o}$ case) and bottom row $\left(2_{i s o}\right.$ case) of Figure 6.4 where HF LS is on right (green histogram) and MC DY is on rihgt (red markers). The sum of apropriately weighted HF LS and MC DY histogram approximate DATA US histgram on right (blue histogram) 
Chapter 6. Muon Isolation Efficiencies

\subsubsection{Calculation of Isolation Efficiency and Error Propaga- tion}

We define:

$N_{1 i s o}=$ number of US $\mu \mu$ events with one leg passing isolation cut

$N_{2 i s o}=$ number of US $\mu \mu$ events passing isolation cut on both legs

$\bar{N}_{2 i s o}=N_{1 i s o}-N_{2 i s o}=$ number of US $\mu \mu$ events pass isolation cut on one leg but failing isolation cut on other leg

efficiency

$$
\epsilon=\frac{N_{2 i s o}}{N_{1 i s o}}
$$

error

$$
\frac{(\Delta \epsilon)^{2}=N_{2 i s o}\left(N_{1 i s o}-N_{2 i s o}\right)}{N_{1 i s o}^{3}}
$$

We use "Bin Likelihood" method to estimate amount of heavy flavor (HF) background in the sample of $U S_{1 i s o}$ and $U S_{2 i s o} 2 \mu$ events. To do the error propagation correctly, we need to use more general formula for the error on efficiency. So, we start from basics. Without loss of generality and for the sake of simplicity we will relabel $N_{1 i s o}, N_{2 i s o}, \bar{N}_{2 i s o}$ as $n, p, f$, respectively.

Then,

efficiency

$$
\epsilon=\frac{p}{(p+f)}
$$

error

$$
(\Delta \epsilon)^{2}=(\Delta p)^{2}\left[\frac{1}{(p+f)}-\frac{p}{(p+f)^{2}}\right]^{2}+(\Delta f)^{2}\left[\frac{p}{(p+f)^{2}}\right]^{2}
$$


Chapter 6. Muon Isolation Efficiencies

$$
=(\Delta p)^{2}\left[\frac{f}{(p+f)^{2}}\right]^{2}+(\Delta f)^{2}\left[\frac{p}{(p+f)^{2}}\right]^{2}
$$

If $p$ and $f$ obey Poisson statistics $\left(i . e .(\Delta p)^{2}=p\right.$ and $(\Delta f)^{2}=f$ we get

$$
(\Delta \epsilon)^{2}=\frac{p f^{2}+f P^{2}}{(p+f)^{4}}=\frac{p f}{(p+f)^{3}}
$$

so we retrieve the formula in (above).

In the case of BG subtraction, $N_{2 i s o} \rightarrow N_{2 i s o}-B_{2 i s o}^{U S}$, where $B_{2 i s o}^{U S}$ is the number of BG US events passing the "both legs isolated" cut and estimated from the "BinLikelihood" fit, It is expressed as $B_{2 i s o}^{U S} \cong c B_{2 i s o}^{L S}$ where $B_{2 i s o}^{L S}$ is the number of $\mathrm{BG}$ LS events passing the "both legs isolated" cut and $c$ is the "Bin-Likelihood" fitting coefficient. The error on $B_{2 i s o}^{U S}$ is

$$
\begin{gathered}
\left(\Delta B_{2 i s o}^{U S}\right)^{2}=c^{2}\left(\Delta B_{2 i s o}^{L S}\right)^{2}+\left(B_{2 i s o}^{L S}\right)^{2}(\Delta c)^{2} \\
=c^{2} B_{2 i s o}^{L S}+\left(B_{2 i s o}^{L S}\right)^{2}(\Delta c)^{2}
\end{gathered}
$$

The error on $N_{2 i s o}$ is

$$
\begin{aligned}
& \left(\Delta N_{2 i s o}\right)^{2}=\left(\Delta N_{2 i s o}\right)^{2}+\left(\Delta B_{2 i s o}^{U S}\right)^{2} \\
& \cong N_{2 i s o}+c^{2} B_{2 i s o}^{L S}+\left(B_{2 i s o}^{L S}\right)^{2}(\Delta c)^{2}
\end{aligned}
$$

and for $\bar{N}_{2 i s o}$,

$$
\left(\Delta \bar{N}_{2 i s o}\right)^{2}=\bar{N}_{2 i s o}+c^{2} \bar{B}_{2 i s o}^{L S}+\left(\bar{B}_{2 i s o}^{L S}\right)^{2}(\Delta c)^{2}
$$

where we neglected small errors on $N_{2 i s o}^{B G-U S}$ and $N \frac{B G-U S}{2 i s o}$ which we take to be errors to function fits (see Fig. 6.4 and Fig. 6.5). 
Chapter 6. Muon Isolation Efficiencies

\subsubsection{Isolation Efficiency and Scale Factors}

In this subsection we present isolation efficiencies for both DATA and MC. Subsequently, we derived the scale factors as the ratio of the isolation efficiency measured in data to one measured in Monte Carlo. For $\mathrm{p}_{T}$ variable, the results are shown in histograms in Figure 6.8 and Table 6.4. For $\mathrm{m}_{\mu \mu}$ variable, the results are shown in histograms in Figure 6.9 and Table 6.5.

\begin{tabular}{l|ccc}
$\mathrm{p}_{T}$ bin & data & $\mathrm{MC}$ & scale factor \\
\hline $0-20$ & $0.907 \pm 0.071$ & $0.957 \pm 0.001$ & $0.948 \pm 0.075 \pm 0.01$ \\
$20-40$ & $0.891 \pm 0.034$ & $0.940 \pm 0.003$ & $0.947 \pm 0.036 \pm 0.005$ \\
$40-60$ & $0.986 \pm 0.008$ & $0.992 \pm 0.001$ & $0.994 \pm 0.008 \pm 0.000$
\end{tabular}

Table 6.4: Isolations efficiencies and scale factors for $3 \mathrm{p}_{T}$ bins: ratio of efficiency measured in the data to the Monte Carlo. The errors in the data are statistical. For scale factors we included sytematical erros discussed in the subsection "Systematics"

\begin{tabular}{l|ccc}
$\mathrm{m}_{\mu \mu}$ bin & data & MC & ratio \\
\hline $0-40$ & $0.948 \pm 0.077$ & $0.960 \pm 0.002$ & $0.987 \pm 0.080 \pm 0.045$ \\
$40-80$ & $0.788 \pm 0.056$ & $0.912 \pm 0.005$ & $0.864 \pm 0.062 \pm 0.055$ \\
$80-120$ & $0.962 \pm 0.011$ & $0.978 \pm 0.001$ & $0.982 \pm 0.012 \pm 0.000$
\end{tabular}

Table 6.5: Isolation efficiencies and scale factors for $3 \mathrm{~m}_{\mu \mu}$ bins: ratio of efficiency measured in the data to the Monte Carlo. The errors in the data are statistical. For scale factors we included sytematical erros discussed in the subsection "Systematics". 
Chapter 6. Muon Isolation Efficiencies
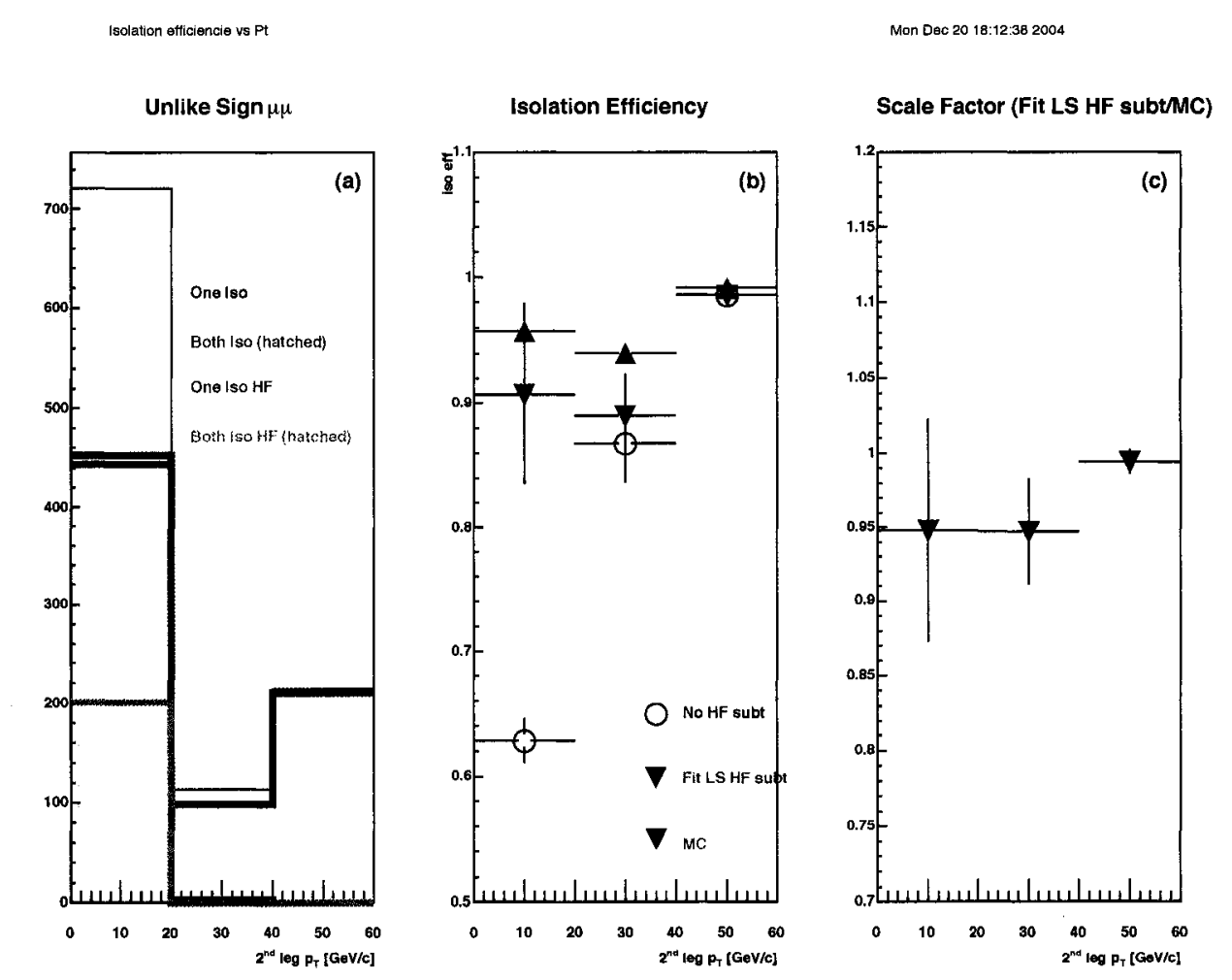

Figure 6.8: Fixed isolation cut of $2 \mathrm{GeV}$ for $\mathrm{p}_{T}<20 \mathrm{GeV}$. LEFT: muon Pt. Unlike Sign (US) in light blue, US one leg iso in medium dark blue, US both leg isolated dark blue. Like Sign (LS) in green. LS one leg iso in medium dark green, LS both leg isolated dark green. CENTER: Isolation efficiency vs $\mathrm{p}_{T}$. RIGHT: Isolation efficiency corrected for LS background. 
Chapter 6. Muon Isolation Efficiencies
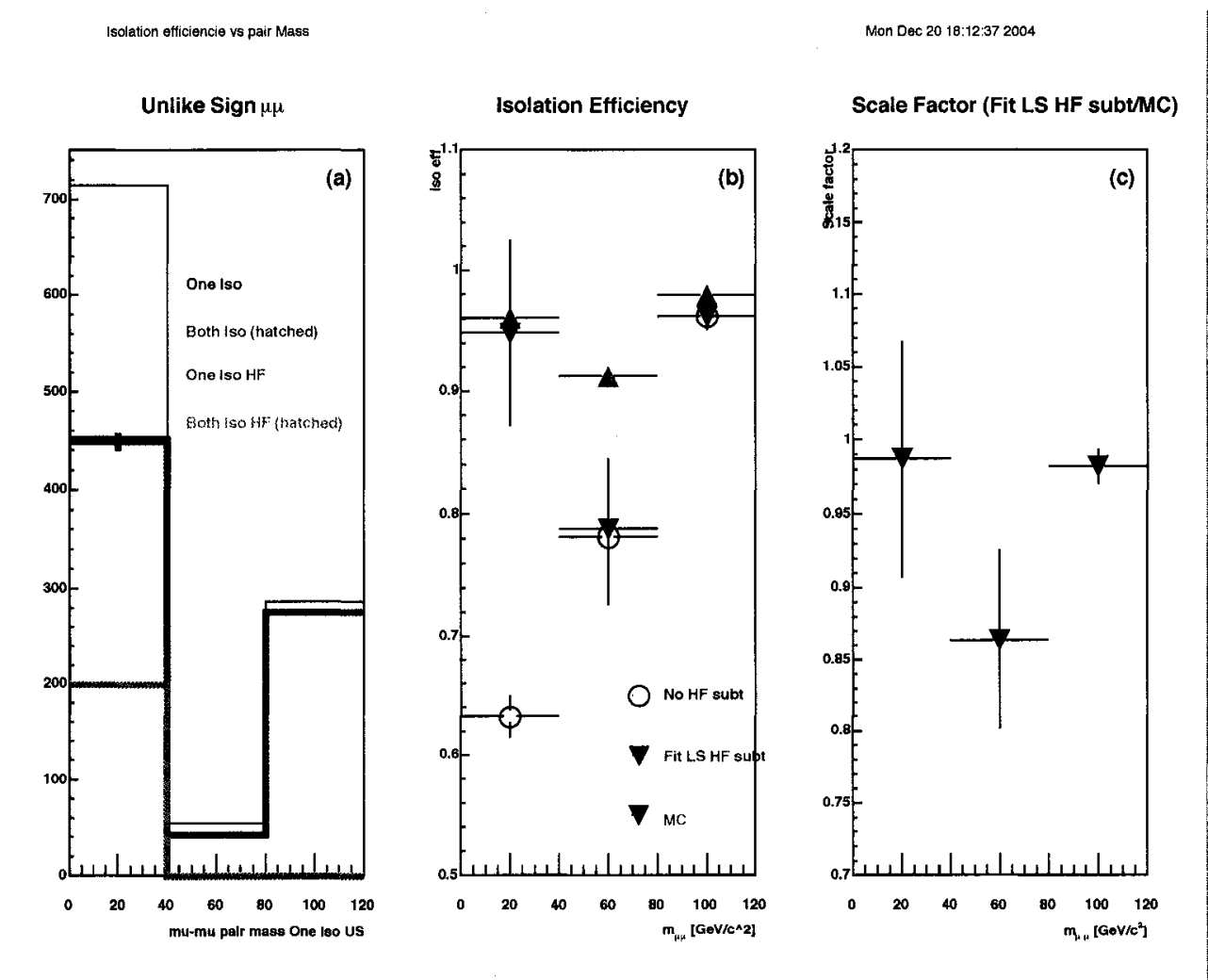

Figure 6.9: Fixed isolation cut of $2 \mathrm{GeV}$ for $\mathrm{p}_{T}<20 \mathrm{GeV}$. LEFT: muon Mass. Unlike Sign (US) in light blue, US one leg iso in medium dark blue, US both leg isolated dark blue. Like Sign (LS) in green. LS one leg iso in medium dark green, LS both leg isolated dark green. CENTER: Isolation efficiency vs $\mathrm{p}_{T}$. RIGHT: Isolation efficiency corrected for LS background. 
Chapter 6. Muon Isolation Efficiencies

\subsubsection{Systematics}

We repeated the study of the isolation efficiency for 3 systematically different scenarios:

- Do not smooth HF LS distribution by fitting a function, but used the distribution "as is".

- Apply all, except for DO, ID cuts on the testing leg

- Redefine isolation to be

- ConeR4Et/ $\mathrm{p}_{T}>0.1$, for $\mathrm{p}_{T}>10 G e V$

- ConeR4Et $>1 \mathrm{GeV}$, otherwise

Apart from the above listed changes, our method was identical to the original. The results for the four scenarios itemized above are given in Figure 6.10, Figure 6.11, Figure 6.12. 

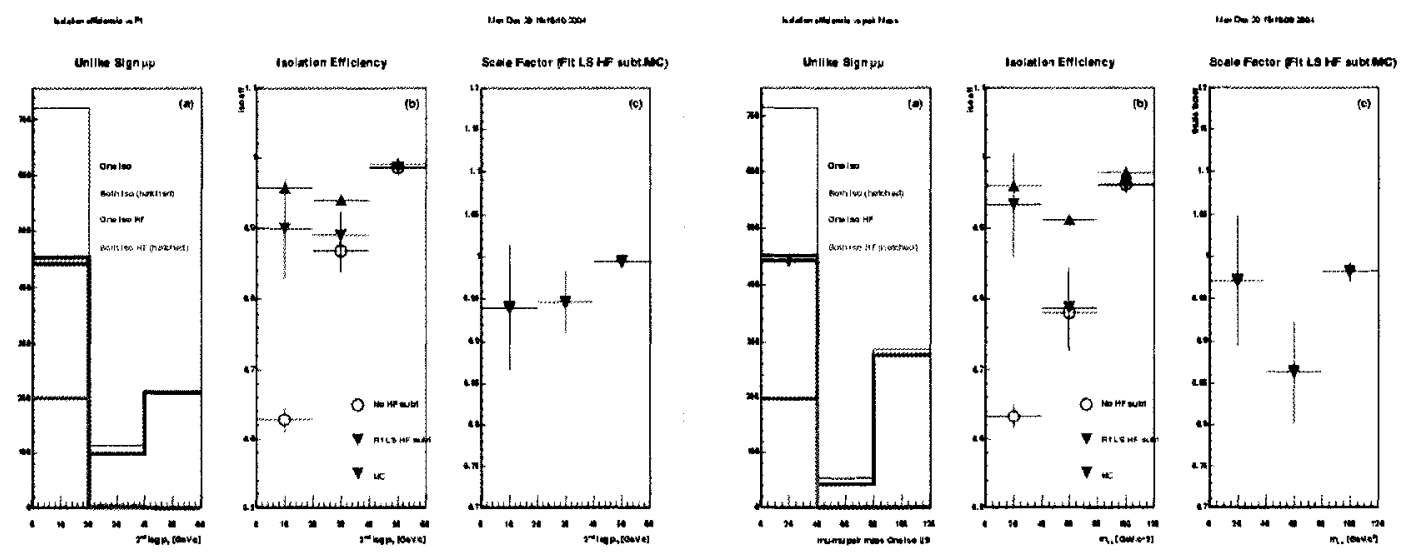

(a) $2^{\text {nd }} \operatorname{leg} \mathrm{p}_{T}$

(b) $\mathrm{m}_{\mu \mu}$

Figure 6.10: No smoothing of HF LS distributions but used the distribution "as is". Results for $\mathrm{p}_{T}$ (left set of three histograms) and results for $\mathrm{m}_{\mu \mu}$ (right three histograms). LEFT: muon Pt. Unlike Sign (US) in light blue, US one leg iso in medium dark blue, US both leg isolated dark blue. Like Sign (LS) in green. LS one leg iso in medium dark green, LS both leg isolated dark green. CENTER: Isolation efficiency vs $\mathrm{p}_{T}$. RIGHT: Isolation efficiency corrected for LS background.
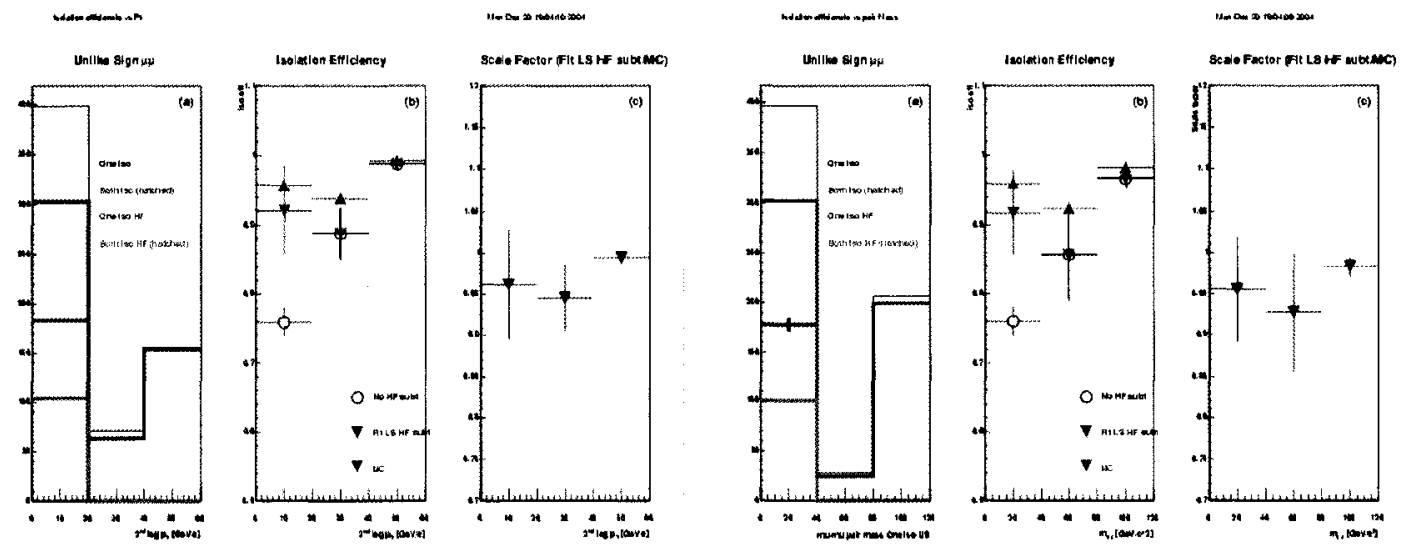

(a) $2^{\text {nd }} \operatorname{leg} \mathrm{p}_{T}$

(b) $\mathrm{m}_{\mu \mu}$

Figure 6.11: Applied all ID cuts, including calorimetry cuts, on both legs. Results for $\mathrm{p}_{T}$ (left set of three histograms) and results for $\mathrm{m}_{\mu \mu}$ (right three histograms). LEFT: muon Pt. Unlike Sign (US) in light blue, US one leg iso in medium dark blue, US both leg isolated dark blue. Like Sign (LS) in green. LS one leg iso in medium dark green, LS both leg isolated dark green. CENTER: Isolation efficiency vs $\mathrm{p}_{T}$. RIGHT: Isolation efficiency corrected for LS background. 

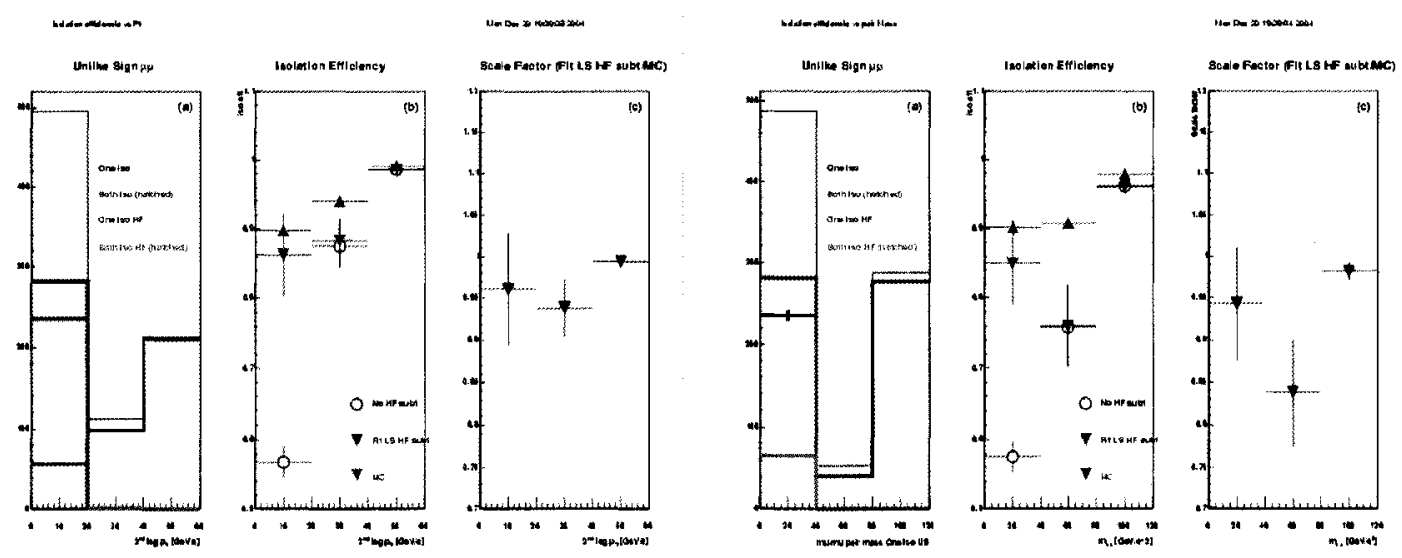

(a) $2^{\text {nd }} \operatorname{leg} \mathrm{p}_{T}$

(b) $\mathrm{m}_{\mu \mu}$

Figure 6.12: Fixed isolation cut of $1 \mathrm{GeV}$ for $\mathrm{p}_{T}<10 \mathrm{GeV}$.Results for $\mathrm{p}_{T}$ (left set of three histograms) and results for $\mathrm{m}_{\mu \mu}$ (right three histograms). LEFT: muon Pt. Unlike Sign (US) in light blue, US one leg iso in medium dark blue, US both leg isolated dark blue. Like Sign (LS) in green. LS one leg iso in medium dark green, LS both leg isolated dark green. CENTER: Isolation efficiency vs $\mathrm{p}_{T}$. RIGHT: Isolation efficiency corrected for LS background. 


\section{Chapter 7}

\section{Measurement of Muon ID Efficiencies in Gen6 Data}

We have determined the identification efficiencies for isolated muons corresponding to the selection criteria for exotics multilepton searches. We use $J / \psi$ and $\mathrm{Z}$ dimuon resonances from SUSY dilepton dataset ediloh, created with Production from cdfsoft2 gen6, to calculate efficiencies of identification for CMU, CMUP, and CMX muons. Our results are in the agreement with the measurement performed on data created with Production cdfsoft2 gen5, documented in the note CDF7210.

In [33], measurement of muon identification efficiencies in data processed with cdfsoft2 gen5 was presented. Since then, approximately $500 \mathrm{pb}^{-1}$ of data were collected at CDF. These data were processed with a new version gen6 of cdfsoft2. In this note, the efficiency measurement of the muon identification (ID) cuts studied in [33], listed in the following subsection, are presented. However, the dataset and the triggers [36] used in this study are very different from the ones used in the work presented in [33]. We discuss the dataset used in this measurement in Section 2. The standard method of using an unbiased muon leg of a dimuon resonance, in our case $J / \Psi$ and 
Chapter 7. Measurement of Muon ID Efficiencies in Gen6 Data

Z, we described in Section 3 and 4. In Section 5 we presented our results. In the Appendix D, we present measurements of ID cuts defined by the Top Group, and we compare our results to the measurements quoted by the Top High $p_{T}$ Muon group in [37]. In the Appendix E, we show plots of efficiency of each cut as a function of $p_{T}$ for both low and high $p_{T}$ region. In the Appendix $\mathrm{F}$, we show plots of efficiency of each cut used by Top Group as a function of $p_{T}$ for both low and high $p_{T}$ region. These cuts don't include total energy (CAL) cut, but include isolation (ISO) cut.

\subsubsection{Muon Identification Cuts}

We are interested in the efficiency of the following ID cuts performed on Cdf-Offline muon:

- Track quality

- $\geq 3$ 5-hit axial stubs, and $\geq 2$ 5-hit stereo stubs

- COT exit radius $>140 \mathrm{~cm}$

- impact parameter to primary vertex, $d_{0}$ :

- if track with SVX hits, then $\left|d_{0}\right|<0.02 \mathrm{~cm}$

- if track with no SVX hits, then $\left|d_{0}\right|<0.20 \mathrm{~cm}$

- if $p_{T}<20 \mathrm{GeV} / c^{2}$, then

- EM energy in tower $<2 \mathrm{GeV}$,

- HAD energy in tower $<3.5+p_{T} / 8 \mathrm{GeV}$

- CAL (EM+HAD) energy in tower $>1 \mathrm{GeV}$,

- if $p_{T}>20 \mathrm{GeV} / c^{2}$, then 
Chapter 7. Measurement of Muon ID Efficiencies in Gen6 Data

- EM energy in tower $<2.0+\theta\left(0.0115 \times\left(p_{T}-100\right)\right) \mathrm{GeV}$

- HAD energy in tower $<6.0+\theta\left(0.028 \times\left(p_{T}-100\right)\right) \mathrm{GeV}$

- CAL (EM+HAD) energy in tower $>1 \mathrm{GeV}$,

- track-stub matching

- CMU stub $\Delta X<3 \mathrm{~cm}$ or $\chi^{2}<9$

- CMP stub $\Delta X<5 \mathrm{~cm}$ or $\chi^{2}<9$

- CMX stub $\Delta X<6 \mathrm{~cm}$ or $\chi^{2}<9$

In the above list of cuts for muons, CMU-muon has a CMU stub, CMUP-muon has both CMU and CMP stubs, and a CMX-muon has a CMX, and $\theta()$ is the step function. Although "CAL" will not be used for gen6 analysis, we list it for the sake of comparison with the results for gen5 listed in CDF7210.

\subsection{Sample}

We selected dimuon events from the SUSY dilepton dataset edil0h, which is created with Production from cdfsoft2 gen6. The data were stored into the Stntuple format, version dev_243, by Ray Culbertson et al. Starting from these stntuples, we select dimuon events that passed DIMUON_CMUCMU4_L2_CMUP6_PT8 or DIMUON_CMU4CMX4_L2_CMUP6_PT8 trigger paths. Although these two dimuon paths have dimuon triggers at L3, they have single muon triggers at L1 (CMUP6_PT4) and L2 (CMUP6_PT8). The only requirement at L3 relevant to ID variables is the matching cut, $\triangle X$, of $15 \mathrm{~cm}$, both for $\mathrm{CMU}$ and CMX. This cut is much loser then the ID matching cut listed in the section 1.1, so that it does not create any L3 trigger bias. Further, dimuon events with the invariant masses in the region of the $J / \psi, \Upsilon$ and $\mathrm{Z}$ were selected. One leg of the pair is required to pass the CMUP L1-L2 trigger 
Chapter 7. Measurement of Muon ID Efficiencies in Gen6 Data

("trigger leg"), and the other leg is used as a sample of muons without a trigger bias. A special care must be taken in the case of $J / \psi$ events, since about 30 percent of $J / \psi$ at CDF originate from $b$ - hadron decays [34]. We cut on the impact parameter of the trigger leg, to exclude $J / \psi$ events with non-prompt vertices. However, we don't cut on the impact parameter in the case of $\Upsilon$, since its production comes predominantly from primary interactions [35]. The invariant mass plots of di-muons form $J / \Psi$ and Z resonances are shown in figures 7.1 and 7.2.

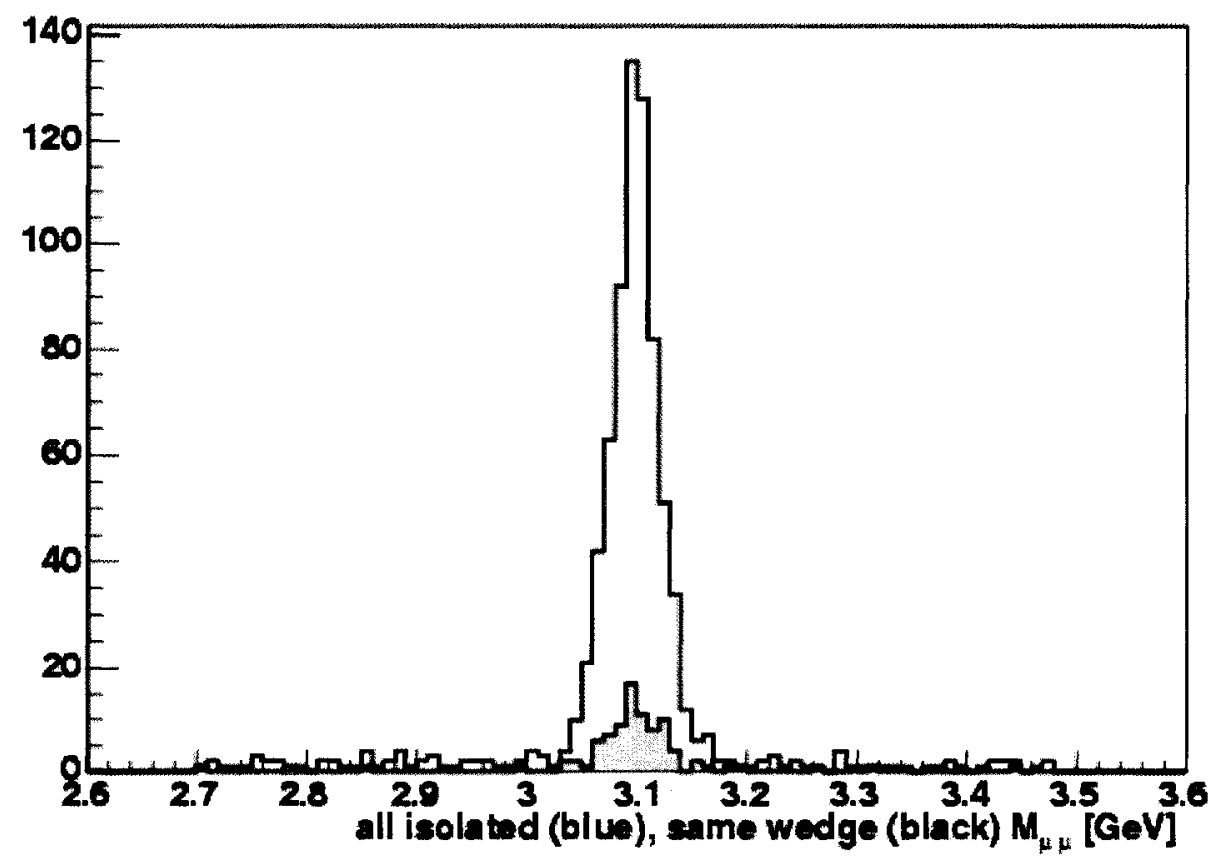

Figure 7.1: JU mass for all isolated dimuons (blue) and dimuons in the same CMU wedge (black, filled). This is only relevant to CMU(P) fiducial muons. Triggering CMUP muon cannot physically be in the same wedge as a CMX fiducial muon.

Finally, the sample of muons is further separated into the three classes of muons considered. ID efficiency is measured for each of the three muon classes, CMU, CMUP and CMX. 

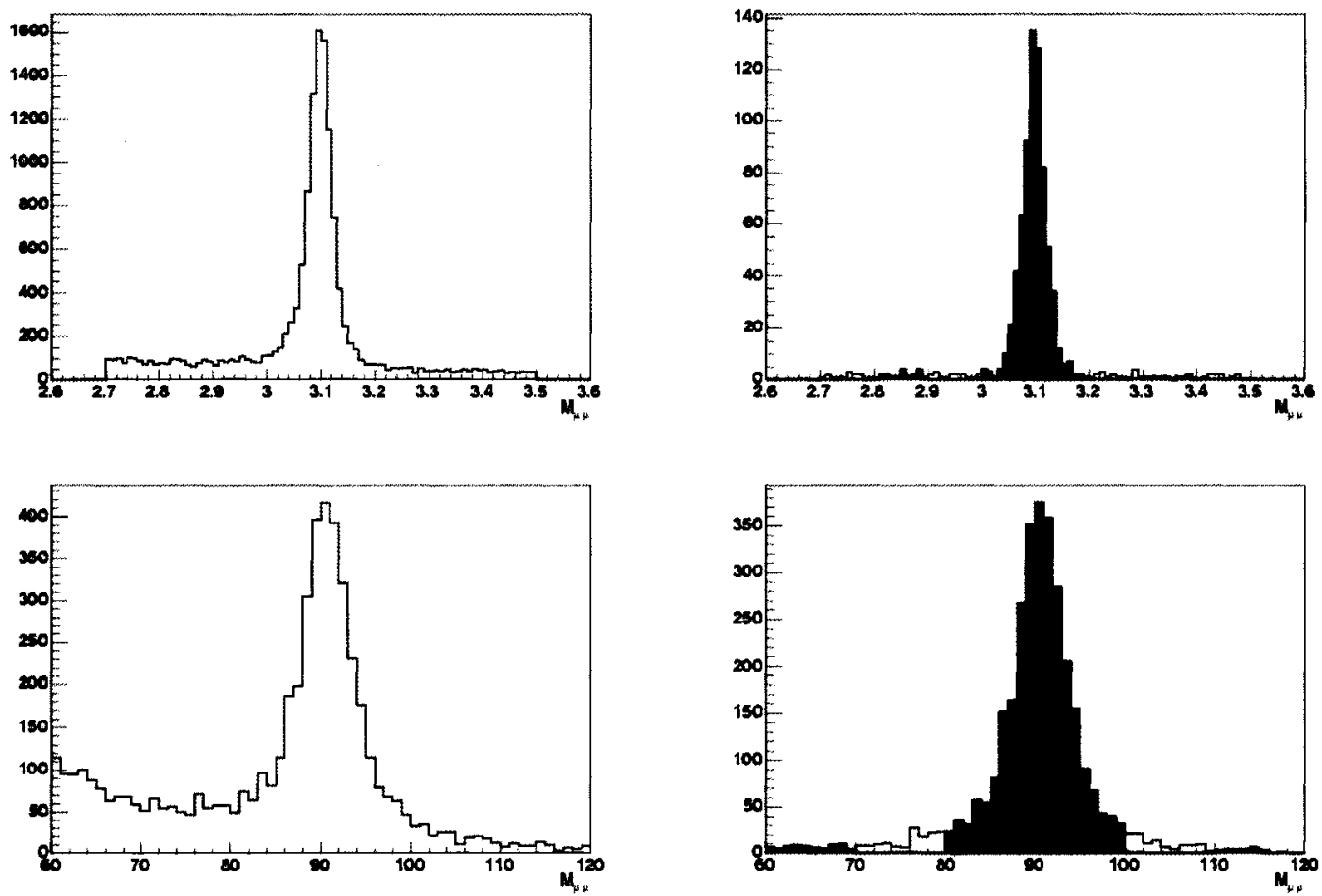

Figure 7.2: $J / \Psi$ (top) and Z (bottom) before (left) and after (right) isolation applied to both legs. On the right, signal (blue) and background (red) regions.

\subsection{Trigger Bias}

We remove a possible trigger bias based on the muon matchbox trigger information stored in the TCMD bank. The event is required to have triggered the L1 CMUP6_PT4 trigger and to have $p_{T}$ at $\mathrm{L} 3>8 \mathrm{GeV} / \mathrm{c}$. The offline reconstructed muon with stub in the same trigger tower as the level 1 trigger muon is identified. The second muon in the event is then unbiased. The identification of the trigger leg is done using stnTrig class [30] of the stnEvent package developed by the UNM group internally [29]. The $p_{T}$ of trigger and other leg are shown in figure 7.3. The distributions show that there is no trigger bias on the testing muon. 
Chapter 7. Measurement of Muon ID Efficiencies in Gen6 Data

\begin{tabular}{|l|ccc|cc|}
\hline & & & gen6 & \multicolumn{2}{c|}{ gen5 CDF7210 } \\
\hline cut & $\epsilon_{\text {low }}(J / \psi)$ & $\epsilon_{\text {low }}(\Upsilon)$ & $\epsilon_{\text {high }}(Z)$ & $\epsilon_{\text {low }}$ & $\epsilon_{\text {high }}$ \\
\hline EM & $1.000 \pm 0.004$ & $1.000 \pm 0.004$ & $0.976 \pm 0.004$ & $0.998 \pm 0.001$ & $0.975 \pm 0.004$ \\
HAD & $1.000 \pm 0.004$ & $0.997 \pm 0.004$ & $0.986 \pm 0.003$ & $0.996 \pm 0.002$ & $0.984 \pm 0.003$ \\
CAL & $1.000 \pm 0.004$ & $1.000 \pm 0.004$ & $1.000 \pm 0.001$ & $1.000 \pm 0.000$ & $0.991 \pm 0.009$ \\
TRK & $1.000 \pm 0.004$ & $1.000 \pm 0.004$ & $1.000 \pm 0.001$ & $0.998 \pm 0.001$ & $1.000 \pm 0.003$ \\
D0 & $0.970 \pm 0.008$ & $0.979 \pm 0.011$ & $0.988 \pm 0.003$ & $0.977 \pm 0.004$ & $0.994 \pm 0.002$ \\
CMUDX & $0.987 \pm 0.006$ & $0.973 \pm 0.010$ & $0.958 \pm 0.005$ & $0.987 \pm 0.004$ & $0.961 \pm 0.005$ \\
ALL & $0.960 \pm 0.010$ & $0.951 \pm 0.014$ & $0.910 \pm 0.008$ & $0.957 \pm 0.006$ & $0.901 \pm 0.008$ \\
\hline
\end{tabular}

Table 7.1: CMU ID efficiencies (gen6 and gen5 reported in CDF7210) for low $p_{T}(<15$ $\mathrm{GeV} / \mathrm{c})$ and $h i g h p_{T}(>20 \mathrm{GeV} / \mathrm{c})$ regions. Efficiencies are listed for each cut applied individually. The efficiency of all the cuts applied together is listed in the last entry, "ALL". This was not the case for gen5, documented in CDFNote 7210.

\subsection{Gen $6 \mu$ ID Efficiencies}

The results of our ID efficiency measurements for CMU, CMUP and CMX muons are shown in tables 7.1, 7.2 and 7.3, respectively. The $p_{T}$ dependence of the ID efficiency measurements for CMU, CMUP and CMX muons are shown in figures 7.4 and 7.5 for low $p_{T}(<15 \mathrm{GeV} / \mathrm{c})$ and high $p_{T}(>20 \mathrm{GeV} / \mathrm{c})$ regions, respectively. In the figures $7.6,7.7,7.8$ and 7.9 we plotted ID variables in LOW $p_{T}$ region after the ID cuts have been applied to all the other ID variables, for CMU, CMUP, and CMX muons. In the figures $7.10,7.11,7.12$ and 7.13 we plotted ID variables in high $p_{T}$ region after the ID cuts have been applied to all the other ID variables, for CMU, CMUP, and CMX muons.

$[\mathrm{htpb}]$ 
Chapter 7. Measurement of Muon ID Efficiencies in Gen6 Data

\begin{tabular}{|l|ccc|cc|}
\hline & & & gen6 & & gen5 CDF7210 \\
\hline cut & $\epsilon_{\text {low }}(J / \psi)$ & $\epsilon_{\text {low }}(\Upsilon)$ & $\epsilon_{\text {high }}(Z)$ & $\epsilon_{\text {low }}$ & $\epsilon_{\text {high }}$ \\
\hline EM & $1.000 \pm 0.006$ & $1.000 \pm 0.006$ & $0.979 \pm 0.005$ & $0.997 \pm 0.002$ & $0.978 \pm 0.004$ \\
HAD & $1.000 \pm 0.006$ & $0.996 \pm 0.005$ & $0.987 \pm 0.004$ & $0.996 \pm 0.003$ & $0.981 \pm 0.004$ \\
CAL & $1.000 \pm 0.006$ & $1.000 \pm 0.006$ & $1.000 \pm 0.002$ & $1.000 \pm 0.002$ & $0.989 \pm 0.011$ \\
TRK & $1.000 \pm 0.006$ & $1.000 \pm 0.006$ & $1.000 \pm 0.002$ & $0.999 \pm 0.001$ & $1.000 \pm 0.003$ \\
D0 & $0.969 \pm 0.010$ & $0.992 \pm 0.011$ & $0.992 \pm 0.003$ & $0.977 \pm 0.005$ & $0.993 \pm 0.003$ \\
CMUDX & $0.990 \pm 0.005$ & $0.984 \pm 0.009$ & $0.953 \pm 0.007$ & $0.987 \pm 0.003$ & $0.969 \pm 0.005$ \\
CMPDX & $1.000 \pm 0.006$ & $0.988 \pm 0.008$ & $0.971 \pm 0.005$ & $0.996 \pm 0.002$ & $0.977 \pm 0.004$ \\
ALL & $0.961 \pm 0.011$ & $0.965 \pm 0.015$ & $0.886 \pm 0.010$ & $0.953 \pm 0.007$ & $0.882 \pm 0.009$ \\
\hline
\end{tabular}

Table 7.2: CMUP ID efficiencies (gen6 and gen5 reported in CDF7210) for low $p_{T}$ $(<15 \mathrm{GeV} / \mathrm{c})$ and $h i g h p_{T}(>20 \mathrm{GeV} / \mathrm{c})$ regions. Efficiencies are listed for each cut applied individually. The efficiency of all the cuts applied together is listed in the last entry, "ALL". This was not the case for gen5, documented in CDFNote 7210.

\begin{tabular}{|l|ccc|cc|}
\hline & & & gen6 & \multicolumn{2}{c|}{ gen5 CDF7210 } \\
\hline cut & $\epsilon_{\text {low }}(J / \psi)$ & $\epsilon_{\text {low }}(\Upsilon)$ & $\epsilon_{\text {high }}$ & $\epsilon_{\text {low }}$ & $\epsilon_{\text {high }}$ \\
\hline EM & $1.000 \pm 0.012$ & $0.991 \pm 0.013$ & $0.973 \pm 0.006$ & $0.995 \pm 0.004$ & $0.981 \pm 0.005$ \\
HAD & $1.000 \pm 0.012$ & $0.982 \pm 0.016$ & $0.983 \pm 0.004$ & $1.000 \pm 0.003$ & $0.975 \pm 0.006$ \\
CAL & $0.978 \pm 0.013$ & $0.991 \pm 0.013$ & $0.983 \pm 0.004$ & $0.988 \pm 0.006$ & $0.987 \pm 0.004$ \\
TRK & $1.000 \pm 0.012$ & $1.000 \pm 0.013$ & $1.000 \pm 0.002$ & $1.000 \pm 0.014$ & $1.000 \pm 0.000$ \\
D0 & $0.978 \pm 0.013$ & $0.945 \pm 0.024$ & $0.990 \pm 0.003$ & $0.987 \pm 0.009$ & $0.991 \pm 0.004$ \\
CMXDX & $1.000 \pm 0.012$ & $1.000 \pm 0.013$ & $0.998 \pm 0.001$ & $0.996 \pm 0.004$ & $0.995 \pm 0.003$ \\
ALL & $0.956 \pm 0.018$ & $0.917 \pm 0.028$ & $0.927 \pm 0.008$ & $0.956 \pm 0.011$ & $0.934 \pm 0.009$ \\
\hline
\end{tabular}

Table 7.3: CMX ID efficiencies (gen6 and gen5 reported in CDF7210) for low $p_{T}(<15$ $\mathrm{GeV} / \mathrm{c})$ and high $p_{T}(>20 \mathrm{GeV} / \mathrm{c})$ regions. Efficiencies are listed for each cut applied individually. The efficiency of all the cuts applied together is listed in the last entry, "ALL". Note, that for gen6 results, we separately measured efficiencies from $J / \psi$ and $\Upsilon$. This was not the case for gen5, documented in CDFNote 7210 . 
Chapter 7. Measurement of Muon ID Efficiencies in Gen6 Data

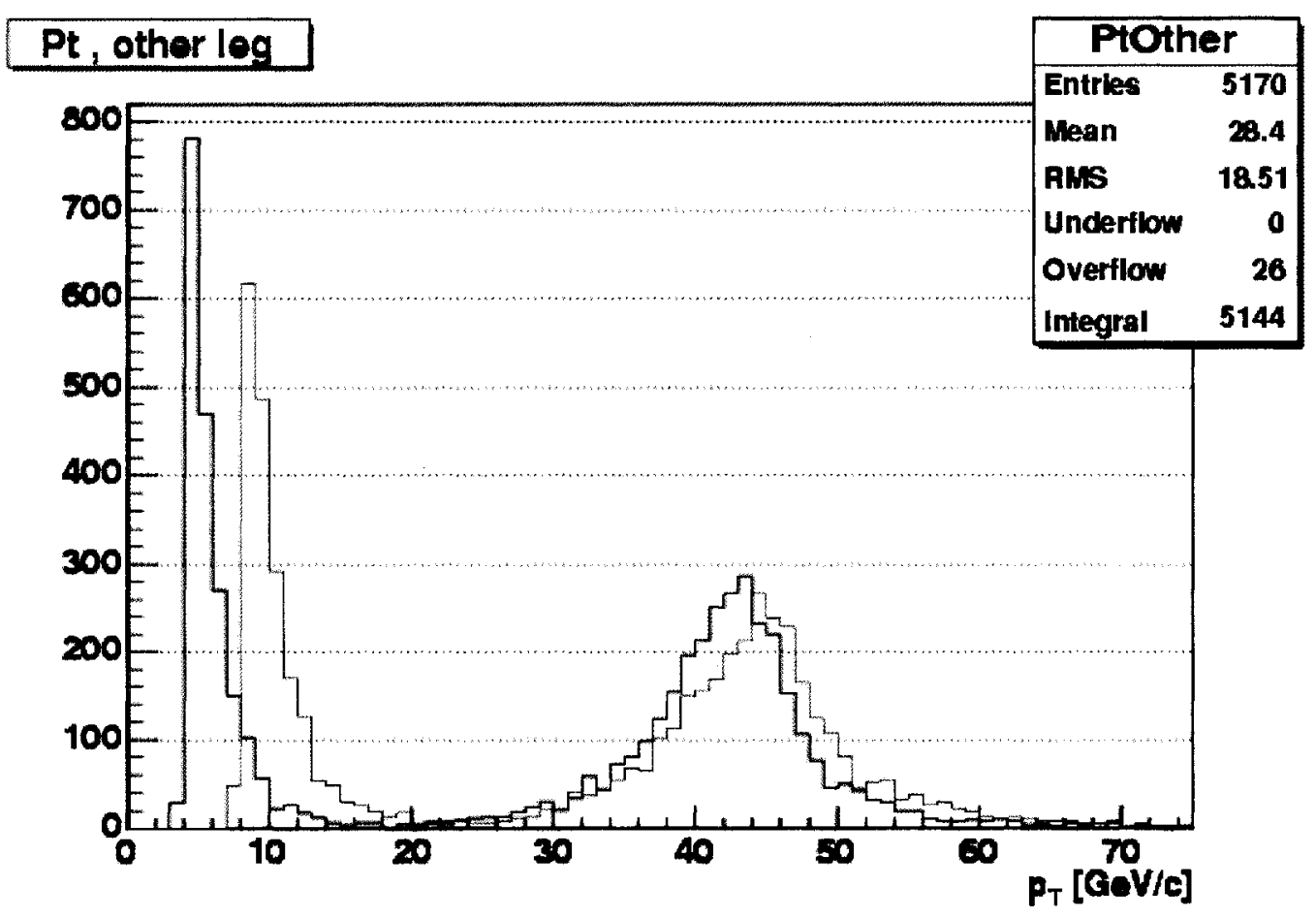

Figure 7.3: $p_{T}$ of the trigger leg (red) and the other leg (blue). 
Chapter 7. Measurement of Muon ID Efficiencies in Gen6 Data
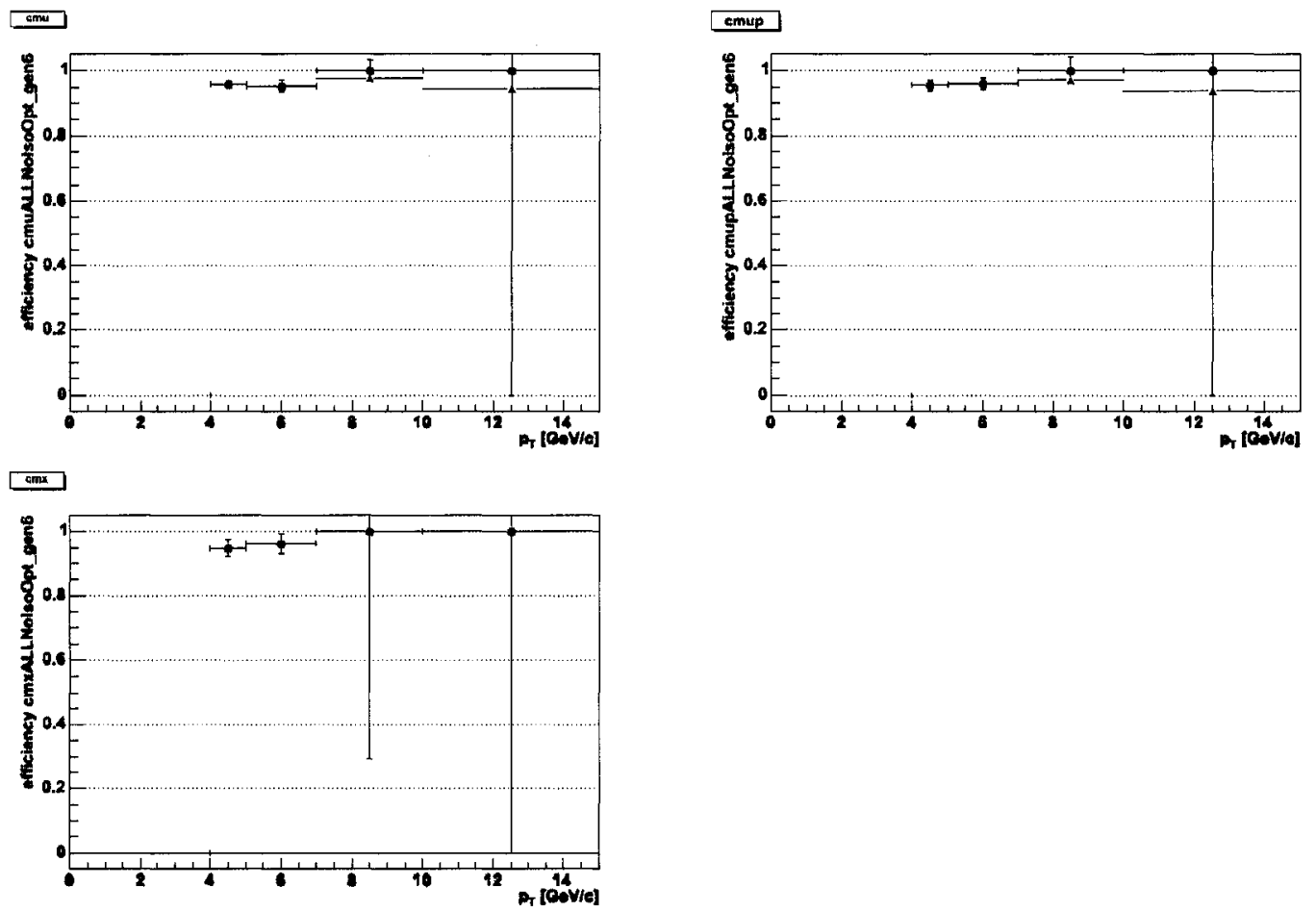

Figure 7.4: CMU, CMUP, and CMX ID efficiencies as a function of $p_{T}$ of other leg (blue circles) and trigger leg (reg triangles), in the low $p_{T}$ region $\left(p_{T}<15 \mathrm{GeV} / c\right)$. 
Chapter 7. Measurement of Muon ID Efficiencies in Gen6 Data
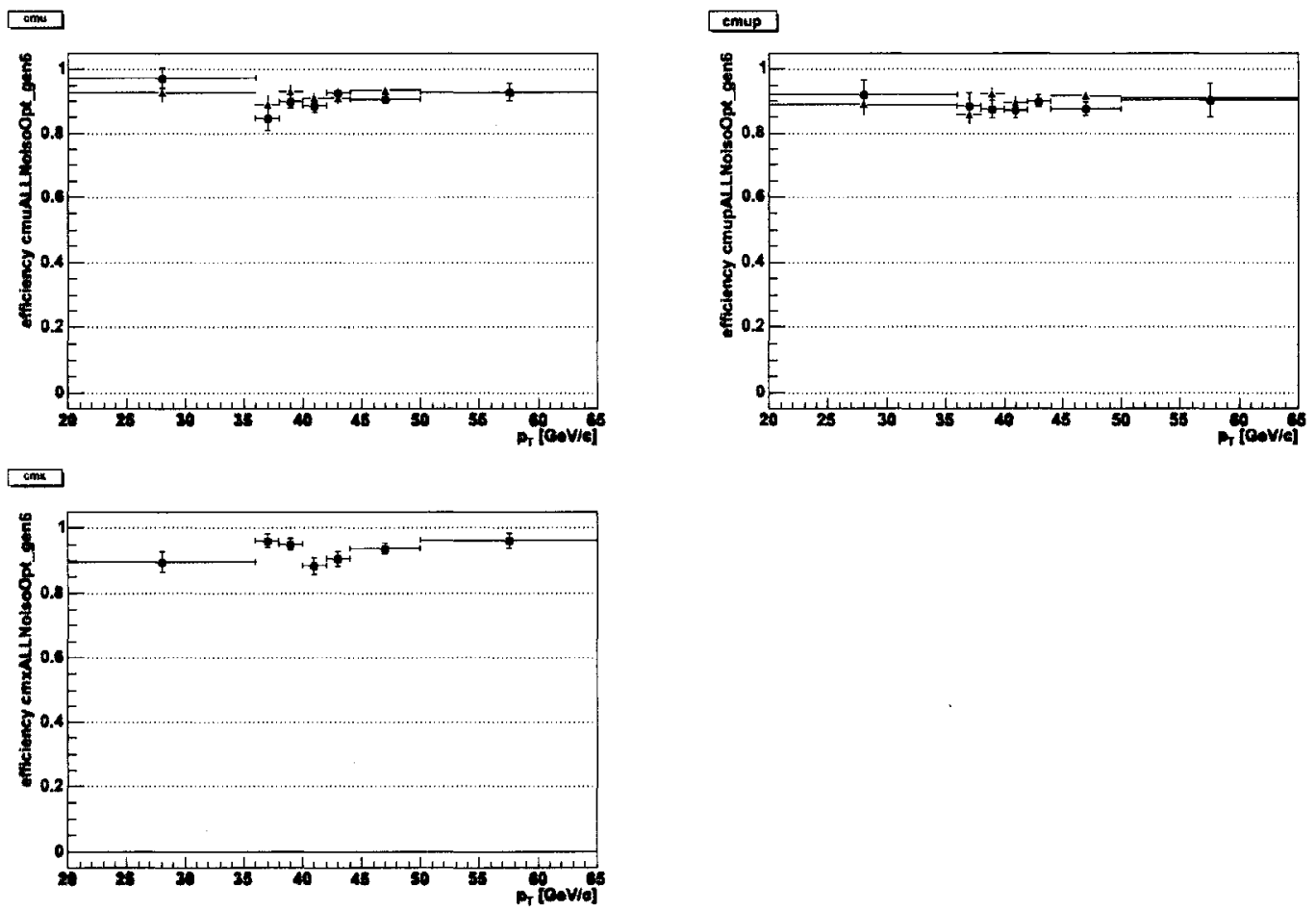

Figure 7.5: CMU, CMUP, and CMX ID efficiencies as a function of $p_{T}$ of other leg (blue circles) and trigger leg (reg triangles), in the high $p_{T}$ region $\left(p_{T}>15 \mathrm{GeV} / c\right)$. 
Chapter 7. Measurement of Muon ID Efficiencies in Gen6 Data
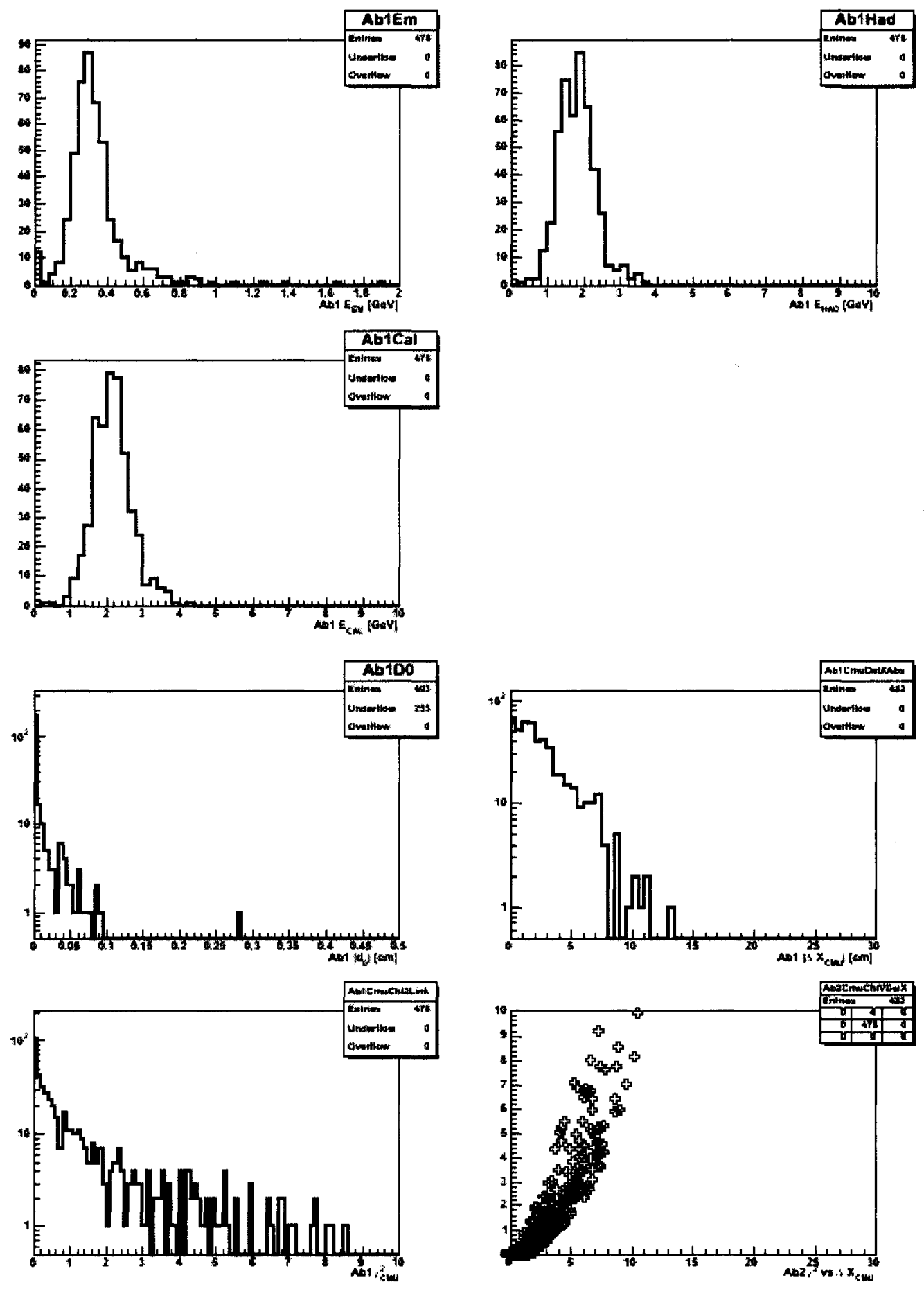

Figure 7.6: In LOW $p_{T}$ region, CMU ID variables with cuts applied on all but the plotted variables. 
Chapter 7. Measurement of Muon ID Efficiencies in Gen6 Data
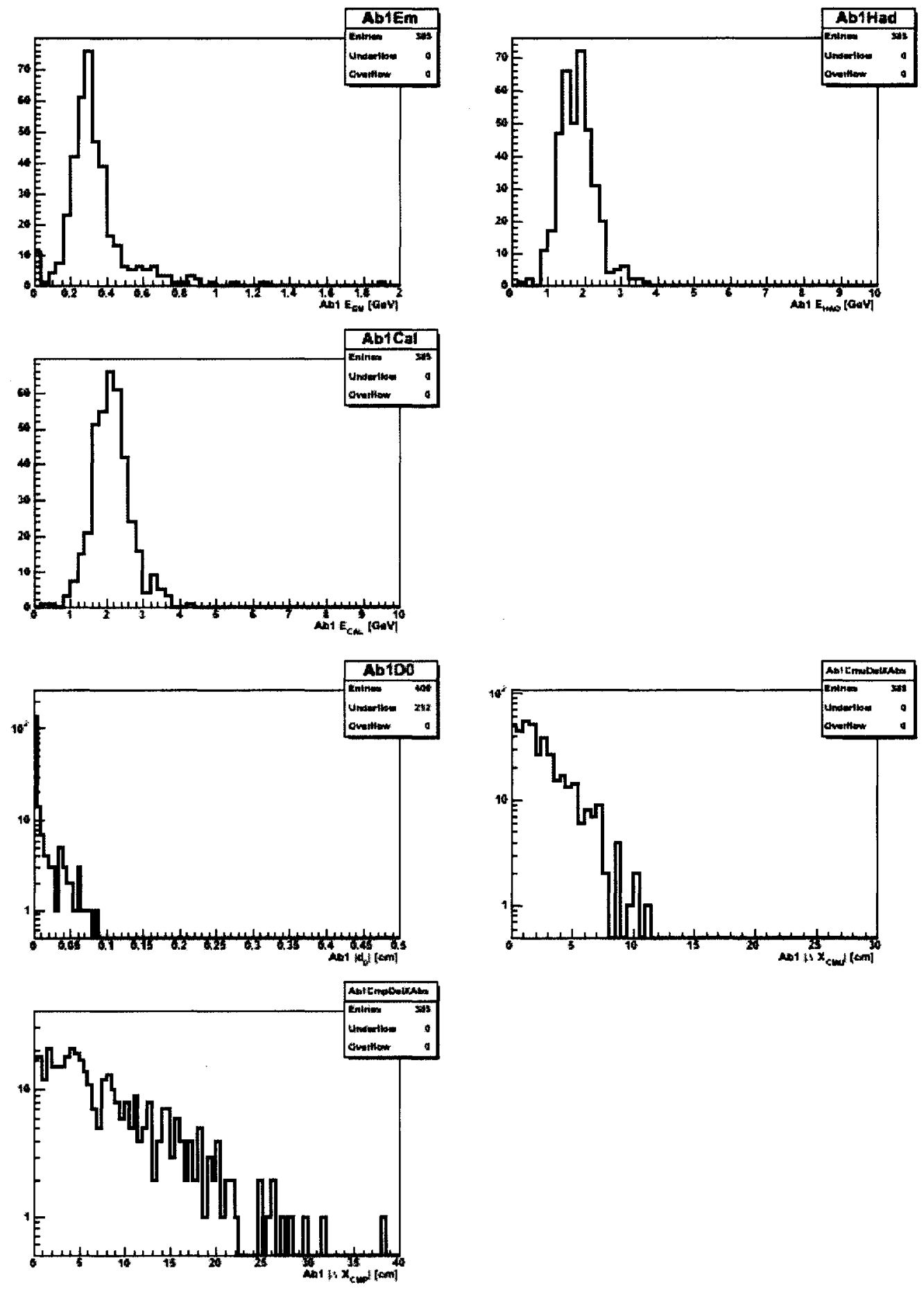

Figure 7.7: In LOW $p_{T}$ region, CMUP ID variables with cuts applied on all but the plotted variables. 
Chapter 7. Measurement of Muon ID Efficiencies in Gen6 Data
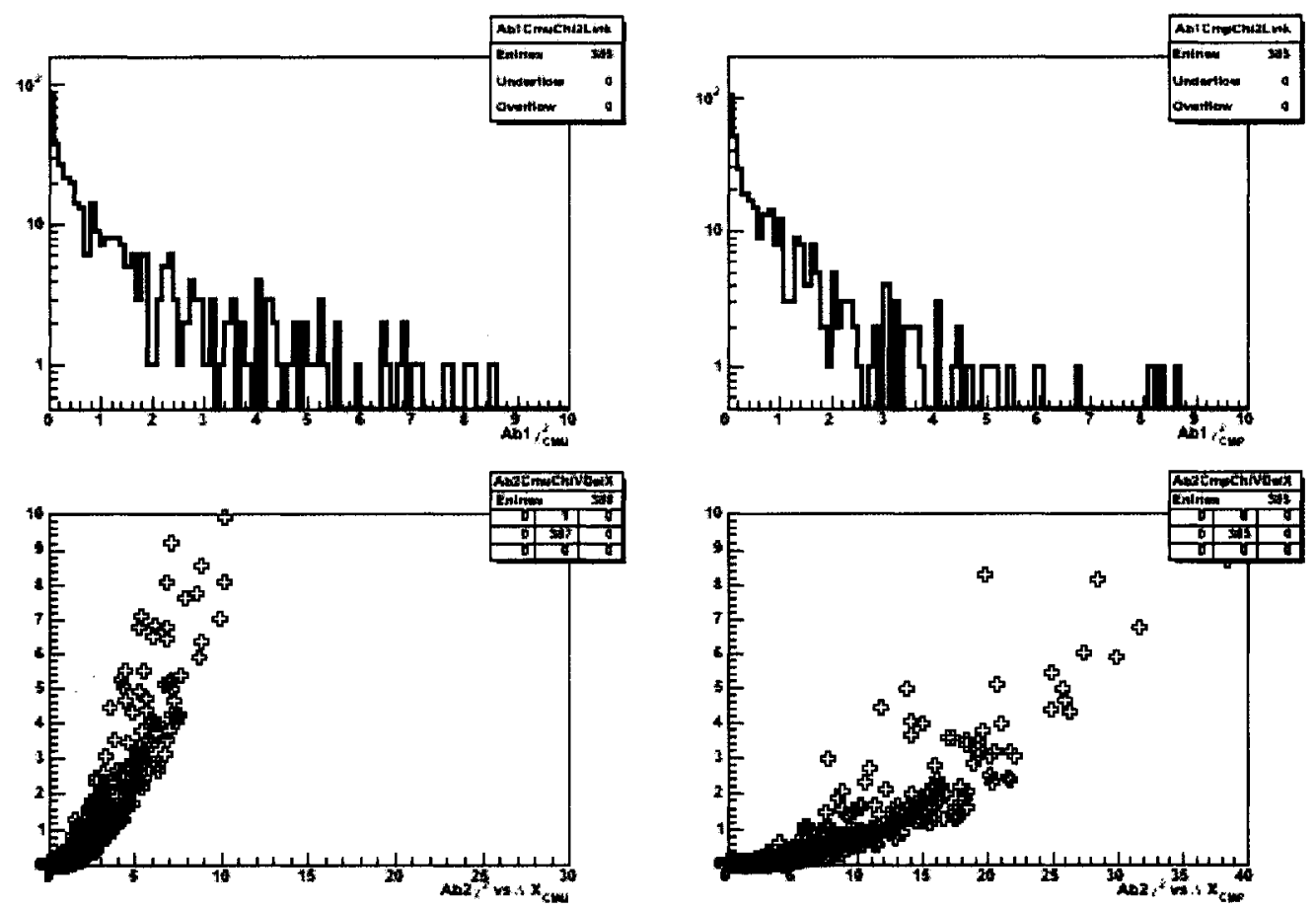

Figure 7.8: In LOW $p_{T}$ region, CMUP ID variables with cuts applied on all but the plotted variables. 
Chapter 7. Measurement of Muon ID Efficiencies in Gen6 Data
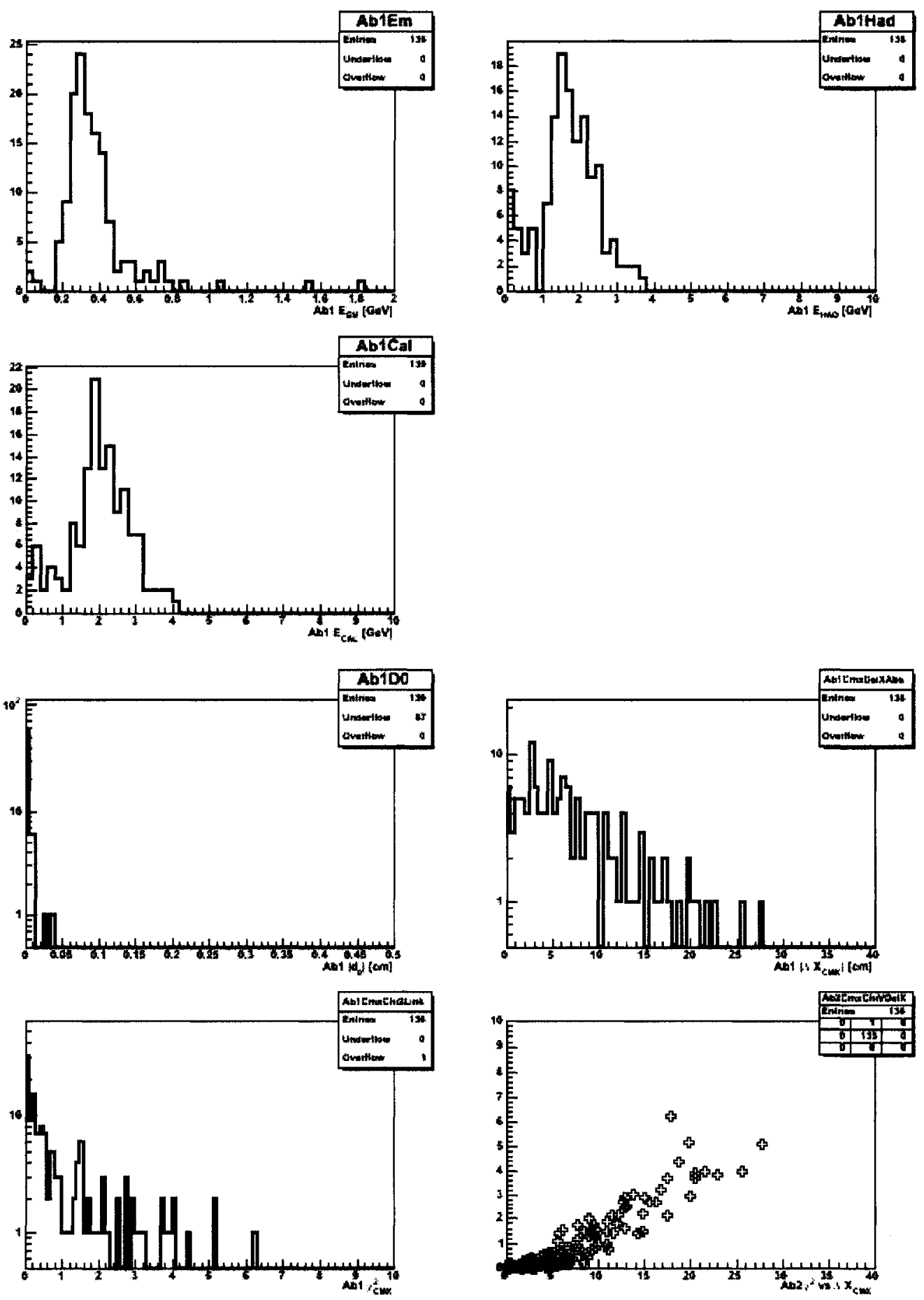

Figure 7.9: In LOW $p_{T}$ region, CMX ID variables with cuts applied on all but the plotted variables. 
Chapter 7. Measurement of Muon ID Efficiencies in Gen6 Data
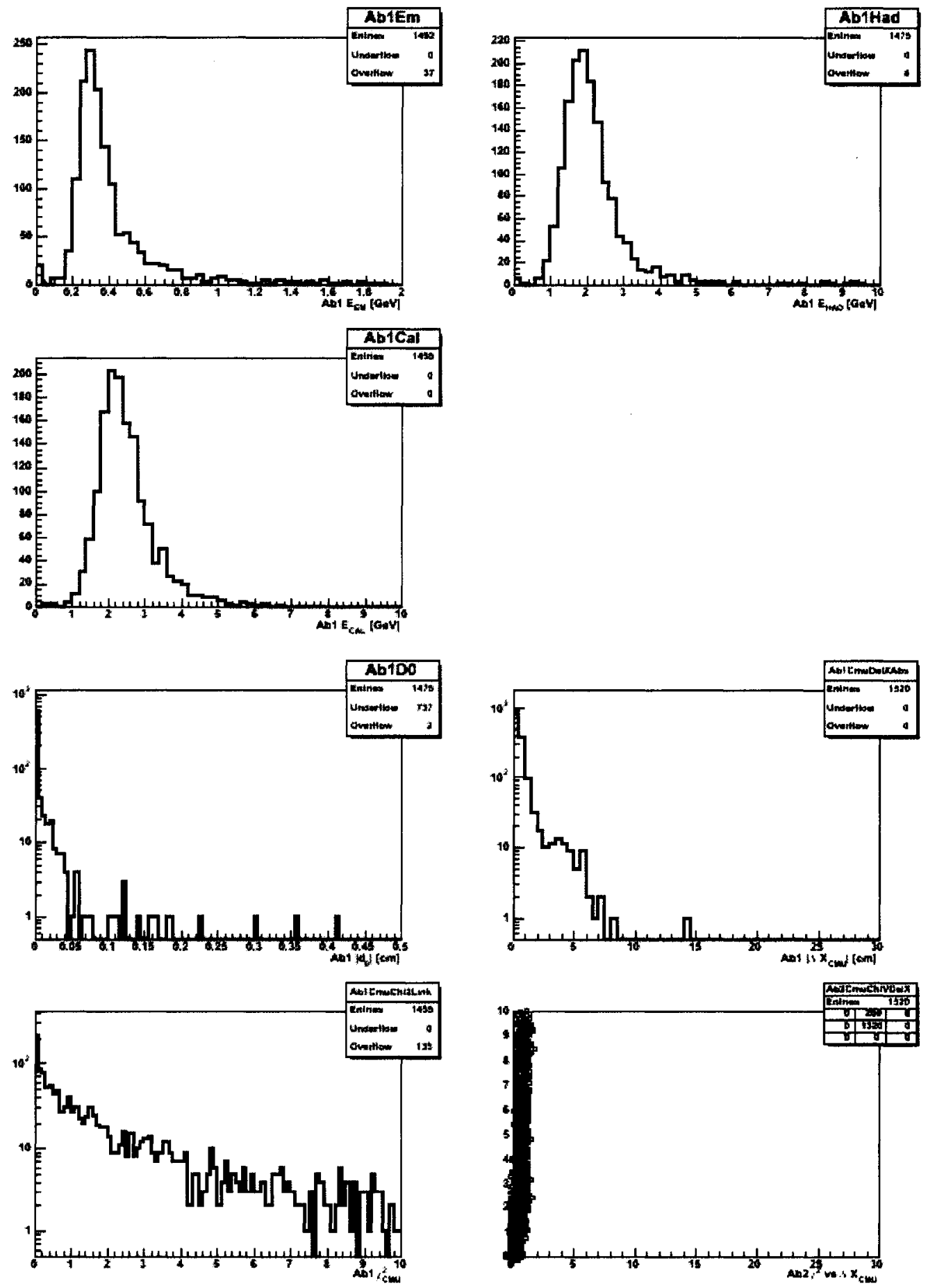

Figure 7.10: In high $p_{T}$ region, CMU ID variables with cuts applied on all but the plotted variables. 
Chapter 7. Measurement of Muon ID Efficiencies in Gen6 Data
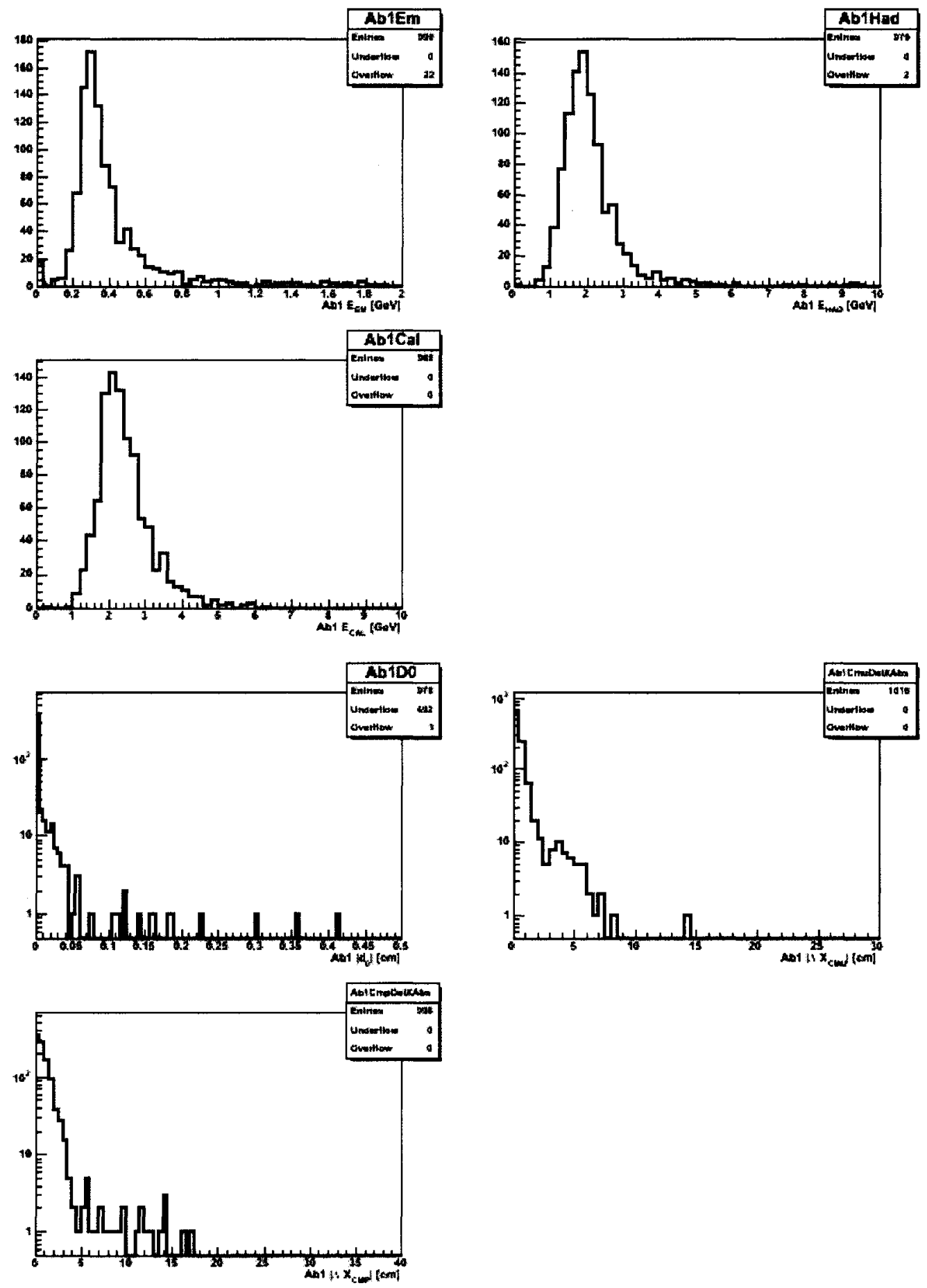

Figure 7.11: In high $p_{T}$ region, CMUP ID variables with cuts applied on all but the plotted variables. 
Chapter 7. Measurement of Muon ID Efficiencies in Gen6 Data
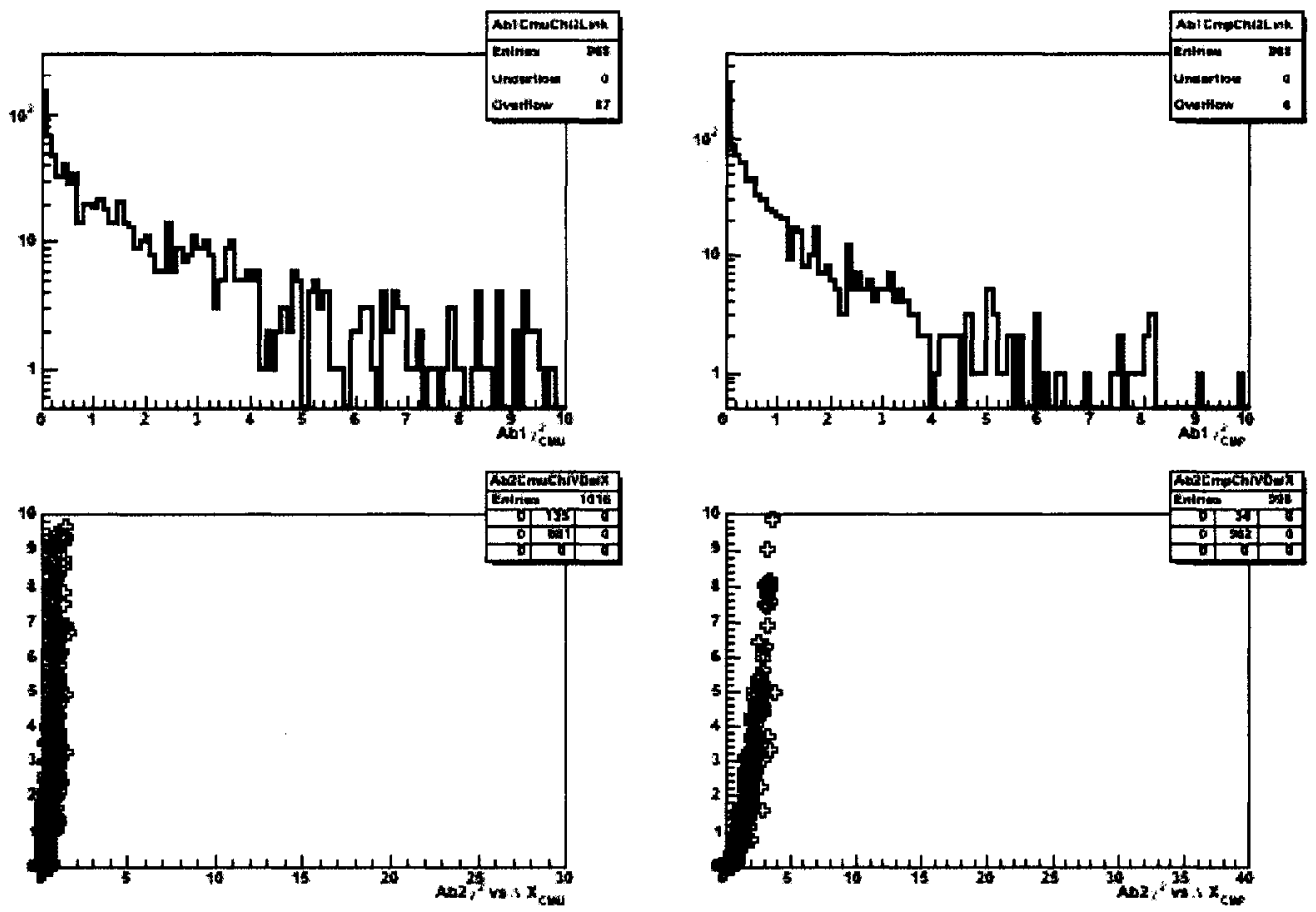

Figure 7.12: In high $p_{T}$ region, CMUP ID variables with cuts applied on all but the plotted variables. 
Chapter 7. Measurement of Muon ID Efficiencies in Gen6 Data
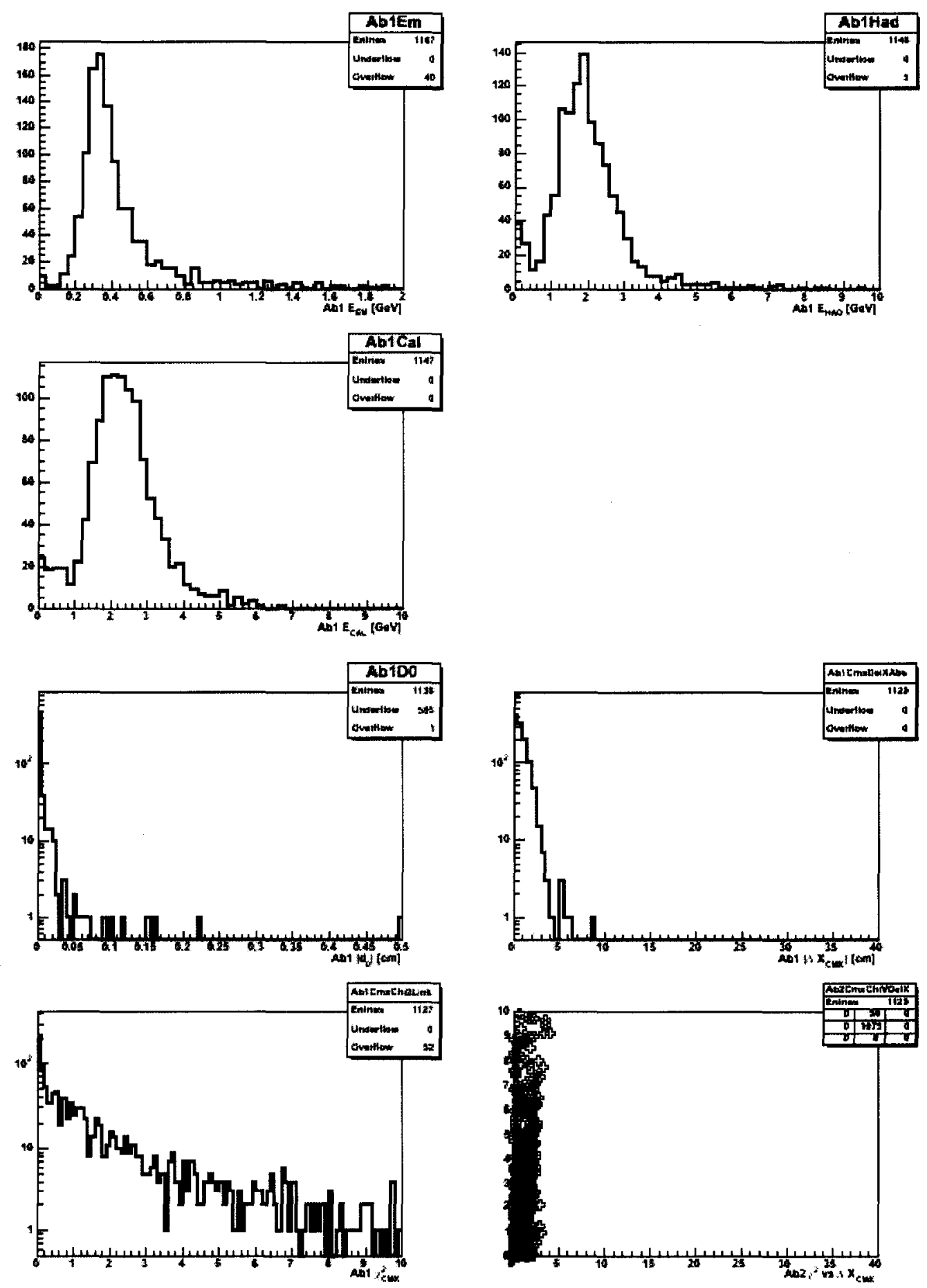

Figure 7.13: In high $p_{T}$ region, CMX ID variables with cuts applied on all but the plotted variables. 
Chapter 7. Measurement of Muon ID Efficiencies in Gen6 Data

\subsection{Summary}

Using SUSY dilepton gen6 data set, edil0h, we were able to measure ID efficiencies of CMU, CMUP, and CMX muons. Our findings, summarized in tables 7.1, 7.2 and 7.3 , show these efficiencies to be within the errors from the ID efficiencies measured for gen5 and documented in CDF7210. Furthermore, the agreement between the results of our measurements of ID cuts used by the Top group and the measurements performed by the Top group for gen6 data provided us with a satisfactory cross check of our methodology. For analysis of gen6 data we intend to use the values of ID efficiencies reported in this document and listed in the tables mentioned above. 


\section{Chapter 8}

\section{Trilepton Analysis}

\subsection{Search Analysis Strategy}

We perform a trilepton analysis using low- $p_{T}$ dimuon triggers discussed in previous chapters. This means that we only searched for the signal in the events that fired these triggers. Other analysis at CDF performed searches in other dilepton channels using different triggers. We conducted a "blind" analysis. This means that we don't look for events with three isolated leptons in the signal region till the very end, after we have understood the backgrounds in the control regions to our satisfaction. We define the signal region in the parameter space as: $M_{\mu \mu}>15 \mathrm{GeV} / c^{2},\left|M_{\mu \mu}-M_{Z}\right|<\mathrm{GeV} / c^{2}$, $\mathbb{E}_{\mathrm{T}}>15 \mathrm{GeV}, N_{j} \leq 1$. We define 19 dilepton/trilepton control regions in the $\mathbb{E}_{\mathrm{T}}$ vs the dimuon mass $M_{\mu \mu}$ vs jet multiplicity. In Figure (ref) we present the 19 dimuon and trilepton control regions we use. In these region SM backgrounds are dominant. SM processes are well understood and so we expect good agreement between expected and observed background. That is to say, in these control regions, the data we observed should agree within uncertainties with processes we expect from SM. There are two major SM background sources for this analysis. One is heavy-flavor and the other 
Chapter 8. Trilepton Analysis

one is Drell-Yan (DY). The low-mass and and/or low- $E_{\mathrm{T}}$ control regions give us a good handle on heavy flavor background, while the control regions with $Z$-mass help us understand DY. When we fully understand the backgrounds in the control regions, we proceed to un-blind ourselves in the signal region. This way our analysis is not statistically biased. We consider all the events that come through dimuon trigger in which the leading muon's transverse momentum is $p_{T} \geq 5 \mathrm{GeV} / c$.

\subsubsection{The observed data}

We analyzed the data collected in the period from March 2002 to February 2006. These data were divided in exotic dilepton edil0d, edil0h and edil0i datasets. For all MC samples and the data processed with version 5.3.3 of CDF software cdfsoft2 we used Stntuples version dev_242. For data processed with version 6.1 .2 of cdfsoft2 we used Stntuples version dev_243. The data luminosity, after removing bad runs [21] and after the application of corrections is $976 \mathrm{pb}^{-1}\left(330 \mathrm{pb}^{-1}\right.$ for edil0d and $646 \mathrm{pb}^{-1}$ for ediloh $+\mathrm{i}$.

\subsubsection{The Backgrounds}

In this analysis various MC generators were used to model the hard scattering of BG processes. PYTHIA 6.2 was used for the parton showering and hadronization. In all cases the CTEQ5L parametrization was used for the parton distribution functions (PDFs). All MC events were simulated using CDF detector simulation software (cdfSim version 5.3.3, which is based on GEANT. To match data, in MC we smear the $1 / p_{T}$ using gaussian distribution with mean 0 and sigma of 0.000475 [26]. In the case of DY $\mathrm{MC}$, we apply a K-factor of 1.4 to account for the difference between the leading and next-to-leading order theoretical cross sections. 
Chapter 8. Trilepton Analysis

There are several SM background (BG) processes which can also yield a final state with three leptons. We must consider each of them, perform our analysis on $\mathrm{MC}$ simulated data that describe them and compare it to the observed data. By studying the kinematic variables of our signal events and our background events, we can devise various cuts that remove most of the background but preserves our signal.

Recall the Feynman diagrams in the Theory chapter of decays of the $\tilde{\chi}_{1}^{ \pm}$and $\tilde{\chi}_{2}^{0}$. Neither of the decay modes to three leptons contain jets. This means that each of the leptons should be well isolated. Coming from associated production of $\tilde{\chi}_{1}^{ \pm} \tilde{\chi}_{2}^{0}$ the leptons should not be particularly back-to-back in $\eta-\phi$ space. Since these leptons are massive objects, we don't expect them to be close together in the detector, but we do expect them to come from the same vertex. The $\tilde{\chi}_{2}^{0}$ should always decay to two opposite sign but same flavor leptons plus LSP.

If the above mentioned cuts are applied together with ID cuts described in previous chapters, most of the BG events will be removed. There will be, however, a number of BG events that pass these cuts: events with three real leptons, and events with two real leptons plus an object that is mistakenly identified as a real lepton. This fake lepton can be a real lepton from the underlying event, a decay in flight, or a hadronic track misidentified as a lepton.

In this trilepton search we study various SM BG processes. Some of them contribute to dilepton BG and some contribute to both dilepton and trilepton BGs. The most important BGs are discussed below.

- $t \bar{t}$

- A top quark will practically always decay $t \rightarrow W^{ \pm} b$ in the SM. If the $W^{ \pm}$decays leptonically, the lepton will be isolated. From $W^{ \pm}$decays we can get two leptons (one from $t$ and one from $\bar{t}$ ). So this is a dilepton BG. If the $b$ decays semileptonically we can get a third lepton, but this type of event should be removed 
by the isolation cut. Also, the production cross section for $t \bar{t}$ is quite small so this background is negligible. We used available dataset atopcb, generated using PYTHIA, with luminosity of $143,156 \mathrm{pb}^{-1}$, which is multiplied by good-run requirement efficiency. In this MC simulation top mass was $m_{t}=178 \mathrm{GeV} / c^{2}$, and cross section $\sigma_{t t}=6.7 \mathrm{pb}^{-1}$.

- $b \bar{b}$ - heavy flavor (HF)

- A typical $b$ decay is $b \rightarrow W^{*} c \rightarrow W^{*}\left(W^{*} d\right)$. Either of two virtual $W$ s has $22 \%$ chance of decaying to an $e$ or $\mu$. In $4.8 \%$ of the decay, both $W^{*}$ will decay leptonically. This is a dilepton BG. If either of $W^{*} \mathrm{~s}$ from $\bar{b}$ decays leptonically then we will have three real leptons in the event. The probability of this is $1 \%$. However, $b$ s are produced at Tevatron with high rate, $b \bar{b}$ is a substantial background. $b$ quark is light compared to the center of mass energy which means it's momentum is large. This means that leptons coming from its decays will be in the direction close to the rest of the products of the decay and therefore will not be isolated. Although MC samples of this process were available to us, we estimate this background from the dilepton data using inverse impact parameter cut. This estimation of this BG requires a more detailed discussion is given in the following subsection.

- Diboson production $\left(Z^{0} Z^{0} a n d W^{ \pm} Z^{0}\right)$

Both the production cross section and branching ratio to three leptons for $Z^{0} Z^{0}$ $(\sigma=1.0 \mathrm{pb}, \mathrm{BR}=0.45 \%$ to $e$ or $\mu)$ and $W^{ \pm} Z^{0}(\sigma=2.5 \mathrm{pb}, \mathrm{BR}=1.44 \%$ to $e$ or $\mu$ ) are small. However, the leptons from either of these processes are very similar to those of our signal, so we must consider this BG. We used the following MC datasets:

- zewk8t: $W Z$ process generated using MADGRAPH, with luminosity of $9,453,013 \mathrm{pb}^{-1}$, which is multiplied by good-run requirement efficiency. 
Chapter 8. Trilepton Analysis

- ZZ process generated using PYTHIA, with luminosity of 535,200 $\mathrm{pb}^{-1}$, which is multiplied by good-run requirement efficiency.

- Diboson production $\left(W^{+} W^{-}\right)$Like in the other diboson BG processes, this one as well has a small cross section. $(\sigma=9.5 \mathrm{pg})$. The only BG with real leptons that this process can give us is the dilepton. However, if there is a fake third lepton, then this process contributes in trilepton BG, too. We used the following MC dataset:

- wtop1w: $W W$ process generated using PYTHIA, with luminosity of 33,849 $\mathrm{pb}^{-1}$, which is multiplied by good-run requirement efficiency.

- Drell-Yan $\left(Z^{0}\right.$ and $\left.\gamma\right)$ production

Due to its extremely large cross section, DY is our dominant BG. It can be split in three categories: $\gamma$ production, and low- and high- $p_{T} Z^{0}$ production. Events with $\gamma$ and low- $p_{T} Z^{0}$ production can be removed with an opening angle cut. Leptons from high- $p_{T} Z^{0}$ production are not back-to-back and these events are removed with a $Z^{0}$ mass window cut. This $\mathrm{BG}$ production is further reduced in our sample by requiring large $E_{\mathrm{T}}$. We use 4 different DY samples, properly merged at the generator level. The reason for this complicated approach is that the $\mathrm{MC}$ sample that has lepton $p_{T}$ requirements looser than our analysis cuts (z8v2gt) has limited statistics, whereas the higher statistics DY MC samples have tighter cuts than ours. The four samples are:

- z8v2gt: DY process generated using PYTHIA allowing decays to electrons, muons and taus, with low $p_{T}$ cuts at generator level $(3.6 \mathrm{GeV}$ for electrons and $4.8 \mathrm{GeV}$ for muons), consistent with our ID cuts given the detector resolution. This sample is used if both of the leptons at generator level have $p_{T}<10 \mathrm{GeV}$. The luminosity of this sample is $1415 \mathrm{pb}^{-1}$, which is divided by the K-factor of 1.4 and multiplied with the good-run requirement 
Chapter 8. Trilepton Analysis

efficiency.

- sexo8t: DY process generated using PYTHIA allowing decays to electrons, muons and taus, with high $p_{T}$ cuts at generator level. This sample is used if both of the leptons at generator level have $p_{T}>10 \mathrm{GeV}$. The luminosity of this sample is $6701 \mathrm{pb}^{-1}$, which is divided by the K-factor of 1.4 and multiplied with the good-run requirement efficiency.

- zewk6m: DY process generated using PYTHIA allowing decays to muons only and $M_{\mu \mu}>20 \mathrm{GeV}$. This sample is used if at least on of the muons at generator level have $p_{T}>10 \mathrm{GeV}$. The luminosity of this sample is 8948 $\mathrm{pb}^{-1}$, which is divided by the K-factor of 1.4 and multiplied with the goodrun requirement efficiency.

- zewk8t: DY process generated using PYTHIA allowing decays to taus only and large $p_{T}$ cuts (10 GeV and $5 \mathrm{GeV}$ for the two leptons). This sample is used if at least on of the leptons at generator level have $p_{T}>10 \mathrm{GeV}$ and $M_{\mu \mu}>20 \mathrm{GeV}$. The luminosity of this sample is $15106 \mathrm{pb}^{-1}$, which is divided by the K-factor of 1.4 and multiplied with the good-run requirement efficiency.

- $W+$ jets

- atopcb: $W+$ parton process generated using ALPEN and HERWIG, with luminosity of $287.6 \mathrm{pb}^{-1}$, which is multiplied by good-run requirement efficiency.

- atopkb: $W+$ charmboson process generated using MADGRAPH, with luminosity of $59,837 \mathrm{pb}^{-1}$, which is multiplied by good-run requirement efficiency.

- atophb: $W+$ bottomboson process generated using ALPEN and HERWIG, with luminosity of $61,554 \mathrm{pb}^{-1}$, which is multiplied by good-run requirement efficiency. 
Chapter 8. Trilepton Analysis

- $\mathrm{DY}+\gamma$ :

For the estimation of this background we use the DY samples presented above, in addition to sexo9t which is the same as sexo8t with a larger luminosity of 22,164 $\mathrm{pb}^{-1}$, which is multiplied by the good-run requirement efficiency and divided by K-factor of 1.4 .

- Cosmics

Cosmic muons are detected as dimuon events in the CDF detector. This types of events are removed using the standard cosmic filter of cdfsoft2 software. The filter is documented elsewhere (ref Cosmic Filter). Residual cosmics are removed by requiring that the $3-\mathrm{D}$ opening angle be less than 178 degrees.

\subsection{Event Selection}

We select events that include either three muons passing the CMUP or CMX requirements of Table (ref muonID) or two muons passing these requirements and a CEM electron passing the requirements of Table (ref electronID). The leptons have to be $\Delta R>0.4$ away from each other, the leading dimuon mass has to be greater than 10.5 $\mathrm{GeV} / c^{2}$, and the three dimensional opening angle between the two leading muons has to be less than 178 degrees. We require primary vertex and redefine impact parameter with respect to this vertex. The absolute value of the $z$ coordinate of the primary vertex has to be less than $60 \mathrm{~cm}$. We count jets in the event if their corrected energy is greater than $20 \mathrm{GeV}$, their electromagnetic fraction (e.m.f) is less than 0.9 and they are $\Delta R>0.4$ away from the leptons in the event. The missing energy, $\mathbb{E}_{\mathrm{T}}$, in the event is corrected for the muons and jets with uncorrected energy greater than $10 \mathrm{GeV}$, e.m.f < 0.9 and $\Delta R>0.4$. 
Chapter 8. Trilepton Analysis

\subsection{Weighing of MC}

To meaningfully compare $\mathrm{MC}$ to data, we weighted the $\mathrm{MC}$ events by appropriate factors so that they correspond to the data luminosity of $976 \mathrm{pb}^{-1}$. Further, these weights have to be multiplied by the trigger efficiency, the lepton ID efficiency scale factors (SF), isolation efficiency (SF), as well as the lepton reconstruction efficiency $(\mathrm{SF})$. Without fully understanding the above efficiencies and their (SF) it is impossible to meaningfully compare BG to data in the control regions. In Chapters 7 and 5 I discuss in detail our measurements of muon ID efficiency and dimuon low- $p_{T}$ trigger efficiencies for gen 6 data. The muon trigger efficiency used for gen 5 data are documented elsewhere [22].

The values of single muon trigger efficiency used for gen5 data are summarized:

$$
\epsilon_{C M U P}=0.889 \pm 0.0056 \epsilon_{C M X}=0.910 \pm 0.007
$$

The values of single muon trigger efficiency used for gen 6 data are:

$$
\epsilon_{C M U P}=0.94 \pm 0.005 \epsilon_{C M X}=0.95 \pm 0.009
$$

An event with only two muons has trigger efficiency

$$
\epsilon_{\text {dimuon }}=\epsilon_{1} \epsilon_{2}
$$

while an event with three muons has trigger efficiencies

$$
\begin{gathered}
\epsilon_{\text {trimuon }}=\sum \epsilon_{i} \epsilon_{j}\left(1-\epsilon_{k}\right)+\epsilon_{1} \epsilon_{2} \epsilon_{3} \\
\epsilon_{\text {trimuon }}=\sum \epsilon_{i} \epsilon_{j}\left(1-\epsilon_{k}\right)+\epsilon_{1} \epsilon_{2} \epsilon_{3}
\end{gathered}
$$

where $\epsilon_{1}, \epsilon_{2}, \epsilon_{3}$ are single muon trigger efficiencies for the three muons. 
Chapter 8. Trilepton Analysis

\begin{tabular}{|c|c|}
\hline \hline Muon type & Muon ID Scale Factor \\
\hline \hline CMUP & $0.9710 \pm 0.0056 \pm 0.0232$ \\
CMX & $1.0222 \pm 0.0077 \pm 0.0258$ \\
\hline
\end{tabular}

Table 8.1: The muon ID scale factors (systematic uncertainty shown second)

\begin{tabular}{|c|c|}
\hline \hline CMUP and CMX $p_{T}\left(\mathrm{GeV} / c^{2}\right)$ & Muon Isolation Scale Factor \\
\hline \hline $0-20$ & $0.948 \pm 0.075 \pm 0.010$ \\
$20-40$ & $0.947 \pm 0.036 \pm 0.005$ \\
$>40$ & $0.994 \pm 0.008 \pm 0.000$ \\
\hline
\end{tabular}

Table 8.2: The muon Isolation scale factors (systematic uncertainty shown second)

\begin{tabular}{|c|c|}
\hline \hline$p_{T}\left(\mathrm{GeV} / c^{2}\right)$ & Electron ID $\times$ Isolation Scale Factor \\
\hline \hline $4-6$ & $0.8 \pm 0.07 \pm 0.010$ \\
$6-8$ & $0.88 \pm 0.06 \pm 0.005$ \\
$8-10$ & $1.06 \pm 0.10 \pm 0.005$ \\
$>10$ & $1.03 \pm 0.02$ (total uncert.) \\
\hline
\end{tabular}

Table 8.3: The electron ID $\mathrm{x}$ Isolation scale factors for gen5 (systemic uncertainty shown second)

\begin{tabular}{|c|c|}
\hline \hline$p_{T}\left(\mathrm{GeV} / c^{2}\right)$ & Electron ID $\times$ Isolation Scale Factor \\
\hline \hline $4-12$ & $0.933 \pm 0.1$ (total uncert.) \\
$12-16$ & $0.999 \pm 0.1$ (total uncert.) \\
$16-20$ & $0.960 \pm 0.1$ (total uncert.) \\
$>20$ & $1.206 \pm 0.1$ (total uncert.) \\
\hline
\end{tabular}

Table 8.4: The electron ID x Isolation scale factors for gen6

The muon ID and muon isolation SF we used are discussed in Chapter 6 and are summarized in Tables 8.1 and 8.2. It turns out that these SF for gen5 and gen6 data are equal within the uncertainty. The electron ID SF (with the electron isolation SF included) for gen5 are given in Table 8.3 and for gen 6 in Table 8.4. 
Chapter 8. Trilepton Analysis

The muon reconstruction efficiency SF for gen 5 are:

$$
S F_{C M U P}^{r e c o}=0.9427 S F_{C M X}^{r e c o}=0.9872
$$

while the muon reconstruction efficiency SF for gen 6 are:

$$
S F_{C M U P}^{r e c o}=0.9325 S F_{C M X}^{\text {reco }}=0.9863
$$

\subsection{Background Estimation}

We use CDF data to estimate HF background and the fakes. Monte Carlo is used for $\mathrm{DY}$, diboson, $t \bar{t}, W+$ jets and $\mathrm{DY}+\gamma$.

\subsubsection{Heavy flavor background estimation}

Since we include muons with very low $p_{T}$ the heavy flavor (HF) is our major background in addition to DY. Unlike for other sources of BG where we use MC to model these processes, in case of heavy flavor (HF) we use data. It is always desirable to use real data over $\mathrm{MC}$ whenever possible, to account for unknown effects that MC misses to model. This background is estimated from a HF-rich set of the CDF dilepton data. HF-rich events are selected by inverting the $d_{0}$ requirement for at least one of the two muons, keeping the rest of the dimuon requirements unchanged. The reversal of the impact parameter cut depends on the presence of silicon hits in the track $\left(\left|d_{0}\right|>0.02\right.$ if $N_{\text {SiHits }}>2$, or $\left|d_{0}\right|>0.2$ if $N_{\text {SiHits }}<2$ ). We then fit the HF+DY dimuon mass to the data, for opposite sing and same-sign dimuons separately as shown in Figures [?] and [?], extracting two sets of weights. These weights are then applied to the HF-rich events, which are fed to our analysis program, passing the same cuts as data (with the exception of the impact parameter cut) in our control regions. Figures ?? to ?? show 
the resulted HF estimation for various low-mass dilepton control regions. These figures support the consistency of the the method, where we observe satisfactory agreement between data and $\mathrm{DY}+\mathrm{HF}$ in all control regions.

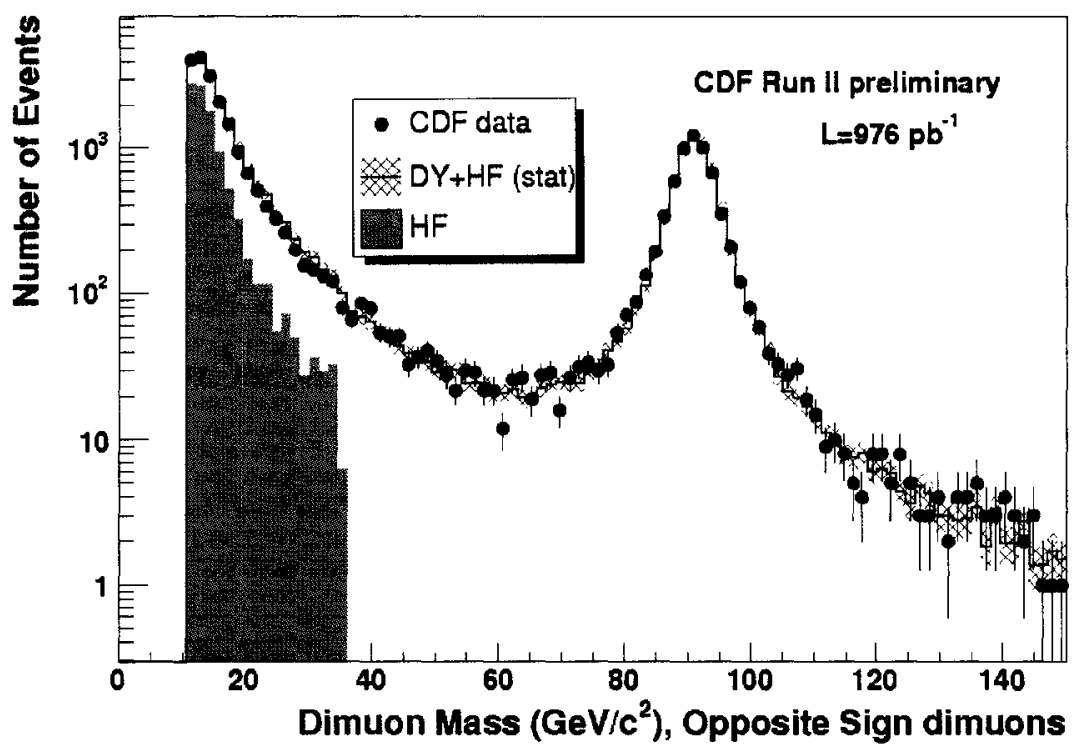

Table 8.5: Fit of di-muon invariant mass for opposite sign pairs.

Because of the limited statistics of the HF-rich dataset, we observe no HF trilepton event in the signal region using both gen5 and gen6 data. Nevertheless, we observe $\mathrm{HF}$ trilepton events in the low $\mathbb{E}_{\mathrm{T}}$ control region (CON_UNM). Given that the $\mathbb{E}_{\mathrm{T}}$ distribution for the dilepton and trilepton events should not be different, we estimate the HF background in the signal region using the following relation

$$
N_{H F, \text { trilep }}^{\mathrm{E}_{\mathrm{T}}>E_{2}} / N_{H F, \text { trilep }}^{\mathrm{E}_{\mathrm{T}}>E_{1}}=N_{H F, \text { dilep }}^{\mathrm{E}_{\mathrm{T}}>E_{2}} / N_{H F, \text { dilep }}^{\mathrm{E}_{\mathrm{T}}>E_{1}}
$$

or

$$
N_{H F, \text { trilep }}^{\mathrm{F}_{\mathrm{T}}>E_{2}}=N_{H F, \text { dilep }}^{\mathrm{E}_{\mathrm{T}}>E_{2}} \times N_{H F, \text { trilep }}^{\mathrm{F}_{\mathrm{T}}>E_{1}} / N_{H F, \text { dilep }}^{\mathrm{E}_{\mathrm{T}}>E_{1}}
$$

where $E_{1}$ is $10.5 \mathrm{GeV}$, and $E_{2}$ is $15 \mathrm{GeV}$, which is our single region minimum $E_{\mathrm{T}}$. 
Chapter 8. Trilepton Analysis

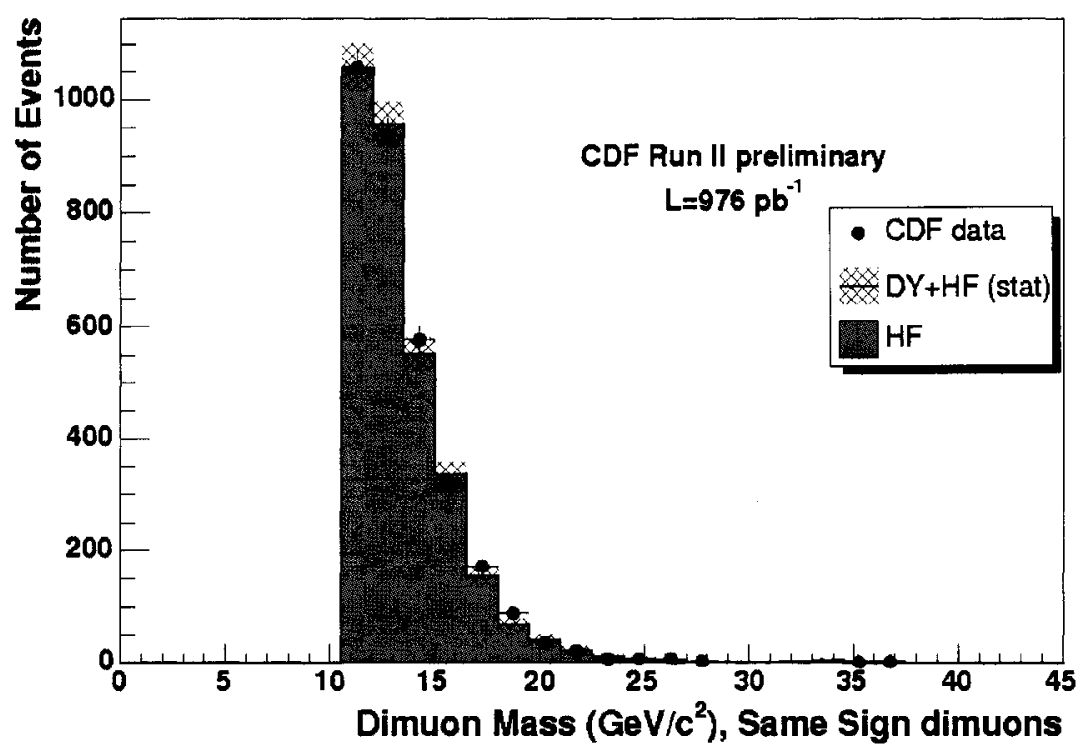

Table 8.6: Fit of di-muon invariant mass for same sign pairs.

We don't see any muon HF-rich gen6 events in region SIG_A2, but we see muon HF-rich events in region SIG_A. As a reminder, we point out that the only difference between control regions SIG_A and SIG_A2 is jet multiplicity. We estimate the HF in region SIG_A2 by assuming that the dimuon HF distribution should not be affected by the jet multiplicity, and using the formula:

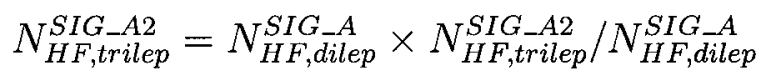

We also don't see any trilepton HF-rich gen6 events in region CON_G, where we estimate the HF assuming the the dimuon and trilepton HF distributions should be alike, and using the formula:

$$
N_{H F, \text { trilep }}^{C O N_{-} G}=N_{H F, \text { dilep }}^{C O N_{-} G} \times N_{H F, \text { trilep }}^{C O N_{-} G} / N_{H F, \text { dilep }}^{C O N_{-} G}
$$


Chapter 8. Trilepton Analysis

\subsubsection{Fakes}

In the case of muons, fake rate is defined as a probability that a track that does not belong to a muon is reconstructed as a muon. In case of electrons, fake rate is defined as a probability that a jet is reconstructed as an electron.

In our estimation of dilepton HF we include the estimation of fakes. For the estimation of trilepton fakes, we first identify the dimuon event and then loop over all tracks (excluding the tracks of the leading two muons) applying the muon fake rate and over all jets applying the electron fake rate. For the fake leptons we require the same cuts as in the case of real ones. We subsequently sum the weights for all events to estimate the trilepton fake background.

\subsubsection{Backgrounds estimated with Monte Carlo}

We run our analysis programs on MC, using the proper luminosity normalization, trigger efficiencies, and lepton-ID, lepton isolation and lepton reconstruction SF. Especially for the trilepton events, to avoid double counting between fakes and MC backgrounds we require that the $\mathrm{MC}$ final leptons are geometrically matched to the generator-level produced real leptons.

Fake estimation does not include the DY $+\gamma$ events where photons are subsequently convert in dileptons. We estimate this background by running our analysis on DY samples demanding that a photon is radiated from one of the two prompt muons. We then remove the requirement that the final leptons are matched to the generator level and count how many DY $+\gamma$ events pass our conversion filter. These events properly weighted give us an estimate on the number of DY $+\gamma$ background events in the trilepton control and signal region.

To every estimated background we associate a statistical error, which is related to 
Chapter 8. Trilepton Analysis

the number of MC events that pass our cuts in a particular control or signal region.

\subsection{Systematics}

The sources of systematic uncertainties for the MC-estimated backgrounds are:

- The luminosity uncertainty of $6 \%$.

- The lepton-ID and isolation scale factors

- Triggers efficiency uncertainty

- Jet energy scale. This source of systematic uncertainty is responsible for migration of events from one control region to another, since the change to jet energy affects both the corrections to the $\mathrm{E}_{\mathrm{T}}$ and the jet-20 multiplicity.

- Parton Distribution Function systematic. We use a previous study [27], and apply $2 \%$ systematic to the BG MC and 1

- Cross section theory systematic of $5-12 \%$ depending on the process, as described in $[27]$.

- The fakes uncertainty of $50 \%$ [28].

- The HF systematic error consists of two parts, an anti-correlated to DY and a non-correlated with DY part. The correlation comes from deriving HF weights from the fit of DY $+\mathrm{HF}$ to data, so that variation on DY weights causes negative variation in $\mathrm{HF}$ weight. The non-correlated error for $\mathrm{HF}$ comes from $2 \%$ uncertainty in the fit DY $+\mathrm{HF}$ to data for fixed DY component and from the HF estimation method systematic. We estimate HF-method systematic by redefining the HF-rich data set using either a requirement of silicon hits for the 
Chapter 8. Trilepton Analysis

muon that has the impact parameter cut inverted, or/and setting cuts on the impact parameter significance. The HF-method systematic is about $25 \%$ in our signal region. The correlated DY+HF systematic is about $8 \%$. The total sytematic uncertainty due to the combined $\mathrm{HF}+\mathrm{DY}$ is then given by

$$
\delta(D Y+H F)=\left[\left(\delta D Y-\delta H F_{c o r r}\right)^{2}+\delta H F_{u n c o r}^{2}\right]^{1 / 2}
$$

In Tables 8.7, 8.7 and 8.7 we show the break-down of systematic errors for the BG, SIG1 and SIG2 in the signal region SIG_A.

\begin{tabular}{|c|c|c|}
\hline \multicolumn{3}{|c|}{ BACKGROUND SYSTEMATICS } \\
\hline \multicolumn{3}{|c|}{ Region SIG_A $\left(\mathscr{G}_{T}>15,15<M_{\mu \mu}<76\right.$ or $\left.M_{\mu \mu}>106, N_{j} \leq 1\right)$} \\
\hline Source & Dileptons & Trileptons \\
\hline Electron scale factors & $+0 \%-0 \%$ & $+1 \%-1 \%$ \\
\hline Muon scale factors & $+8 \%-7 \%$ & $+3 \%-3 \%$ \\
\hline Luminosity & $+3 \%-3 \%$ & $+1 \%-1 \%$ \\
\hline Trigger efficiency & $+0.5 \%-0.5 \%$ & $+0.1 \%-0.1 \%$ \\
\hline PDF & $+1 \%-1 \%$ & $+0.4 \%-0.4 \%$ \\
\hline Theoretical cross sections & $+3 \%-3 \%$ & $+1 \%-1 \%$ \\
\hline Jet energy scale & $+0.5 \%-0.2 \%$ & $+0 \%-0.02 \%$ \\
\hline Total MC syst. & $+9 \%-8 \%$ & $+4 \%-4 \%$ \\
\hline Fakes estimation & $+0.03 \%-0.03 \%$ & $+24 \%-24 \%$ \\
\hline $\mathrm{HF}$ estimation & $+11 \%-11 \%$ & $+5 \%-5 \%$ \\
\hline Total (with correlations) & $+12 \%-12 \%$ & $+24 \%-24 \%$ \\
\hline
\end{tabular}

Table 8.7: Systematic errors for the backgrounds in SIG A region. 
Chapter 8. Trilepton Analysis

\begin{tabular}{|c||c|c|}
\hline \multicolumn{3}{|c|}{ SIGNAL-1 SYSTEMATICS } \\
\hline \hline Region SIG_A $\left(\boldsymbol{H}_{\boldsymbol{T}}>\mathbf{1 5}, \mathbf{1 5}<\boldsymbol{M}_{\boldsymbol{\mu} \boldsymbol{\mu}}<\mathbf{7 6}\right.$ or $\left.\boldsymbol{M}_{\boldsymbol{\mu} \boldsymbol{\mu}}>106, \boldsymbol{N}_{j} \leq 1\right)$ \\
\hline Source & Dileptons & Trileptons \\
\hline Electron scale factors & $+0 \%-0 \%$ & $+6 \%-6 \%$ \\
Muon scale factors & $+11 \%-10 \%$ & $+13 \%-13 \%$ \\
Luminosity & $+6 \%-6 \%$ & $+6 \%-6 \%$ \\
Trigger efficiency & $+0.9 \%-0.9 \%$ & $+0.5 \%-0.5 \%$ \\
PDF & $+1 \%-1 \%$ & $+1 \%-1 \%$ \\
Theoretical cross sections & $+7 \%-7 \%$ & $+7 \%-7 \%$ \\
Jet energy scale & $+0.09 \%-0.39 \%$ & $+0 \%-0 \%$ \\
\hline \hline Total & $+14 \%-14 \%$ & $+18 \%-17 \%$ \\
\hline
\end{tabular}

Table 8.8: Systematic errors for the SIG1 point in SIG A region.

\begin{tabular}{|c|c|c|}
\hline \hline \multicolumn{3}{|c|}{ SIGNAL-2 SYSTEMATICS } \\
\hline \hline Region SIG_A $\left(\boldsymbol{F}_{\mathbf{T}}>\mathbf{1 5}, \mathbf{1 5}<\boldsymbol{M}_{\mu \mu}<\mathbf{7 6}\right.$ or $\left.\boldsymbol{M}_{\boldsymbol{\mu} \mu}>\mathbf{1 0 6}, \mathbf{N}_{\mathbf{j}} \leq \mathbf{1}\right)$ \\
\hline Source & Dileptons & Trileptons \\
\hline Electron scale factors & $+0 \%-0 \%$ & $+6 \%-6 \%$ \\
Muon scale factors & $+11 \%-11 \%$ & $+15 \%-14 \%$ \\
Luminosity & $+6 \%-6 \%$ & $+6 \%-6 \%$ \\
Trigger efficiency & $+0.9 \%-0.9 \%$ & $+0.5 \%-0.5 \%$ \\
PDF & $+1 \%-1 \%$ & $+1 \%-1 \%$ \\
Theoretical cross sections & $+7 \%-7 \%$ & $+7 \%-7 \%$ \\
Jet energy scale & $+0.4 \%-0.2 \%$ & $+0.9 \%-0 \%$ \\
\hline \hline Total & $+15 \%-15 \%$ & $+19 \%-18 \%$ \\
\hline \hline
\end{tabular}

Table 8.9: Systematic errors for the SIG2 point in SIG A region. 
Chapter 8. Trilepton Analysis

\subsection{Control Regions}

Our signal region in the parameter space is defined as: 3 leptons, large $\mathbb{E}_{\mathrm{T}}$, exclusion of the $Z$ peak and low jet multiplicity. We remain blind at the signal region, and we define 22 control regions in phase-space (with minimal signal presence), where we compare the observed data to the SM event-yield prediction.

\begin{tabular}{|c||c|c||c|c|}
\hline \hline \multicolumn{4}{|c|}{ Control and Signal Regions Yields } \\
\hline \multirow{2}{*}{ Control Region } & \multicolumn{2}{|c|}{ Dimuons } & \multicolumn{2}{c|}{ Trileptons } \\
\cline { 2 - 5 } & Expected & Observed & Expected & Observed \\
\hline CON_Z & $6423 \pm 644$ & 6347 & $3 \pm 1$ & 4 \\
CON_UNM & $26595 \pm 1451$ & 26295 & $16 \pm 6$ & 16 \\
CONEE & $219 \pm 23$ & 253 & $0.3 \pm 0.1$ & 0 \\
CONF & $23 \pm 3$ & 28 & $0.02 \pm 0.01$ & 0 \\
CON_G & $8557 \pm 851$ & 8205 & $6 \pm 2$ & 8 \\
CON_H & $29 \pm 10$ & 50 & $0.04 \pm 0.02$ & 0 \\
CON_I & $5267 \pm 528$ & 5185 & $2 \pm 1$ & 2 \\
CON_J & $69 \pm 8$ & 71 & $0.05 \pm 0.03$ & 1 \\
SIG_A & $300 \pm 38$ & 297 & $0.4 \pm 0.1$ & 1 \\
SIG_A2 & $24 \pm 6$ & 23 & $0.03 \pm 0.01$ & 0 \\
\hline \hline
\end{tabular}

Table 8.10: Expected and observed number of dilepton and trilepton events in our control regions. We observe one trilepton event in the signal region SIG_A

In Table 8.10 we summarize our comparison in the control and signal region. We show $M_{\mu \mu}$ and $E_{\mathrm{T}}$ distributions for these regions in the following subsections. 
Chapter 8. Trilepton Analysis

\subsubsection{Control Region CON_Z}

In this region, we require unlike sing dimuon pairs, and dimuon mass between 76 and $106 \mathrm{GeV}$. Figures 8.1 to 8.4 show the $M_{\mu \mu}$ and $\mathrm{E}_{\mathrm{T}}$ distributions for data, SM predicted BG, and SUSY signal for dilepton and trilepton case.
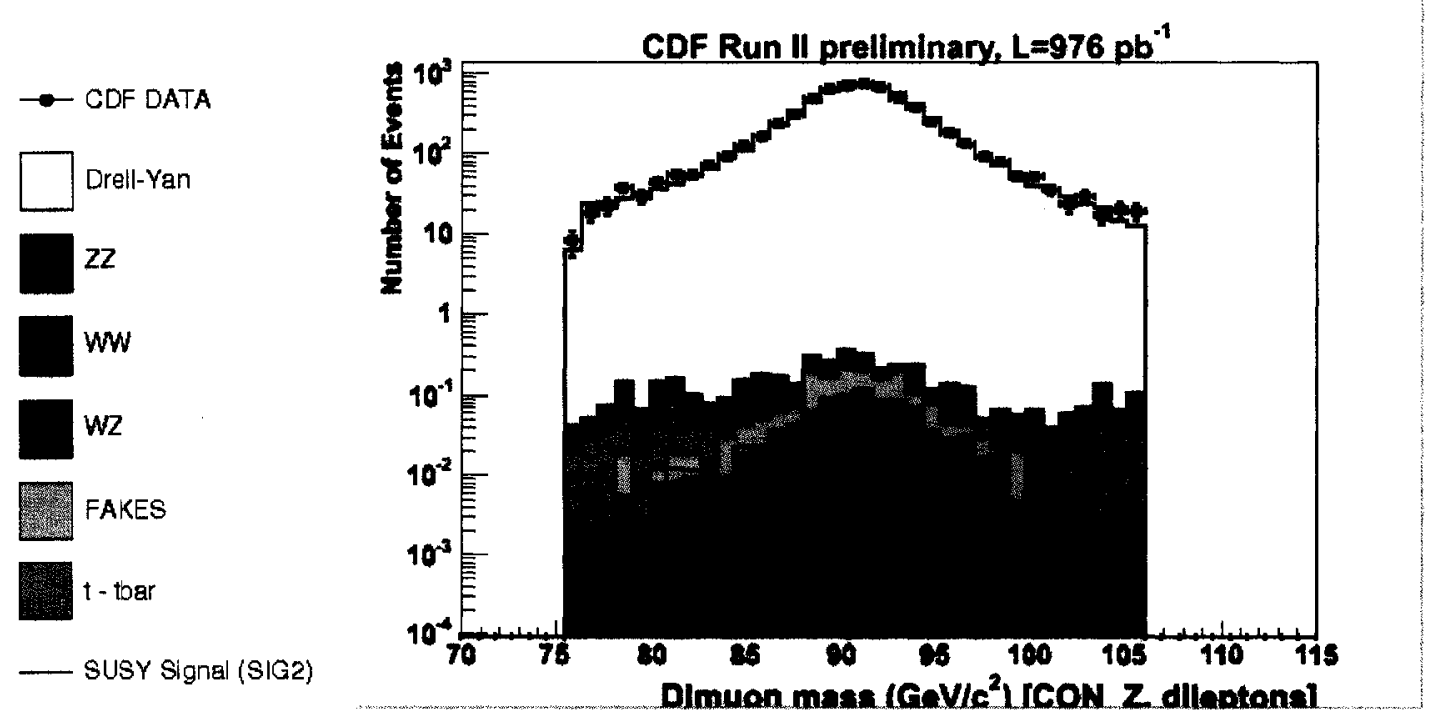

Figure 8.1: Dimuon mass for data, SM prediction, and SUSY MC signal in region CON_Z 
Chapter 8. Trilepton Analysis
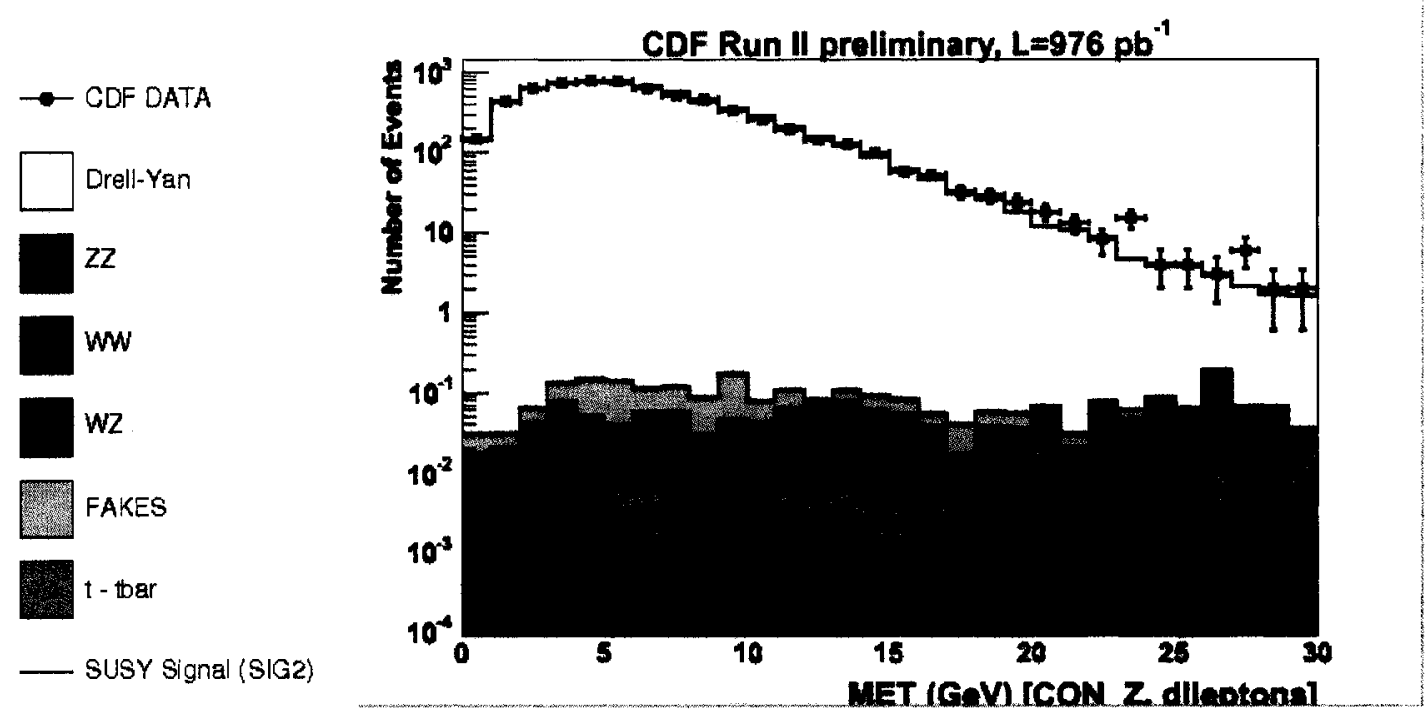

Figure 8.2: $\mathbb{E}_{\mathrm{T}}$ for data, SM prediction, and SUSY MC signal in region CON_Z
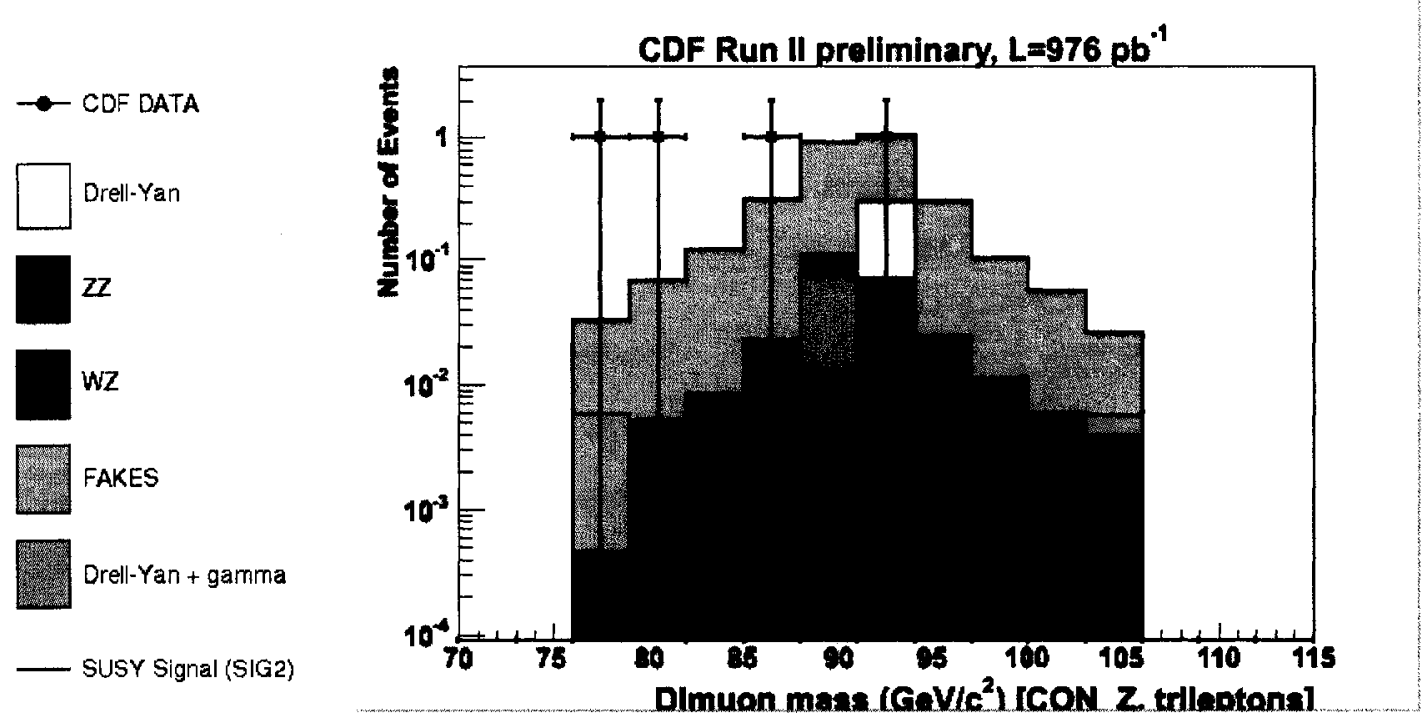

Figure 8.3: Dimuon mass for data, SM prediction, and SUSY MC signal in region CON_Z 
Chapter 8. Trilepton Analysis
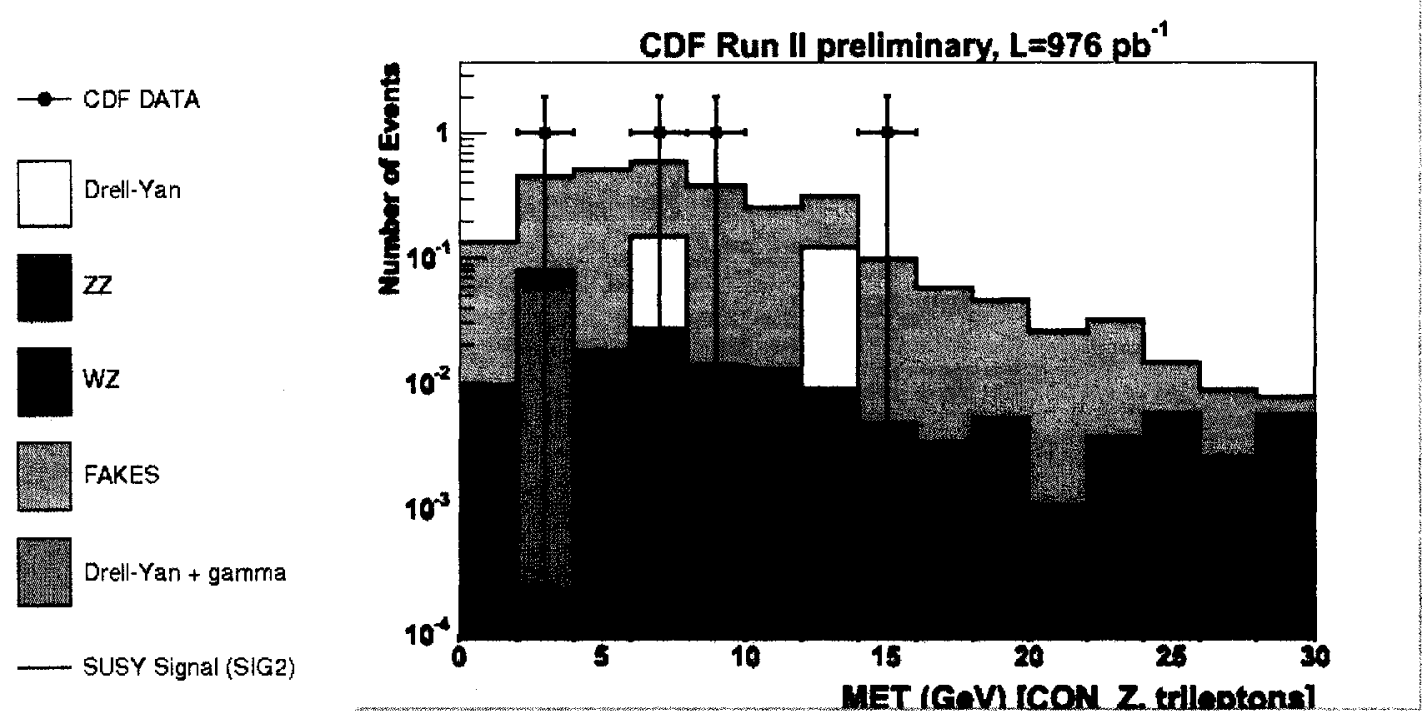

Figure 8.4: $\mathbb{E}_{\mathrm{T}}$ for data, SM prediction, and SUSY MC signal in region CON_Z 
Chapter 8. Trilepton Analysis

\subsubsection{Control Region CON_UNM}

In this region, we only require $\mathrm{E}_{\mathrm{T}}<10 \mathrm{GeV}$. Figures 8.5 to 8.8 show the $M_{\mu \mu}$ and $\mathrm{E}_{\mathrm{T}}$ distributions for data, SM predicted BG, and SUSY signal for dilepton and trilepton case.
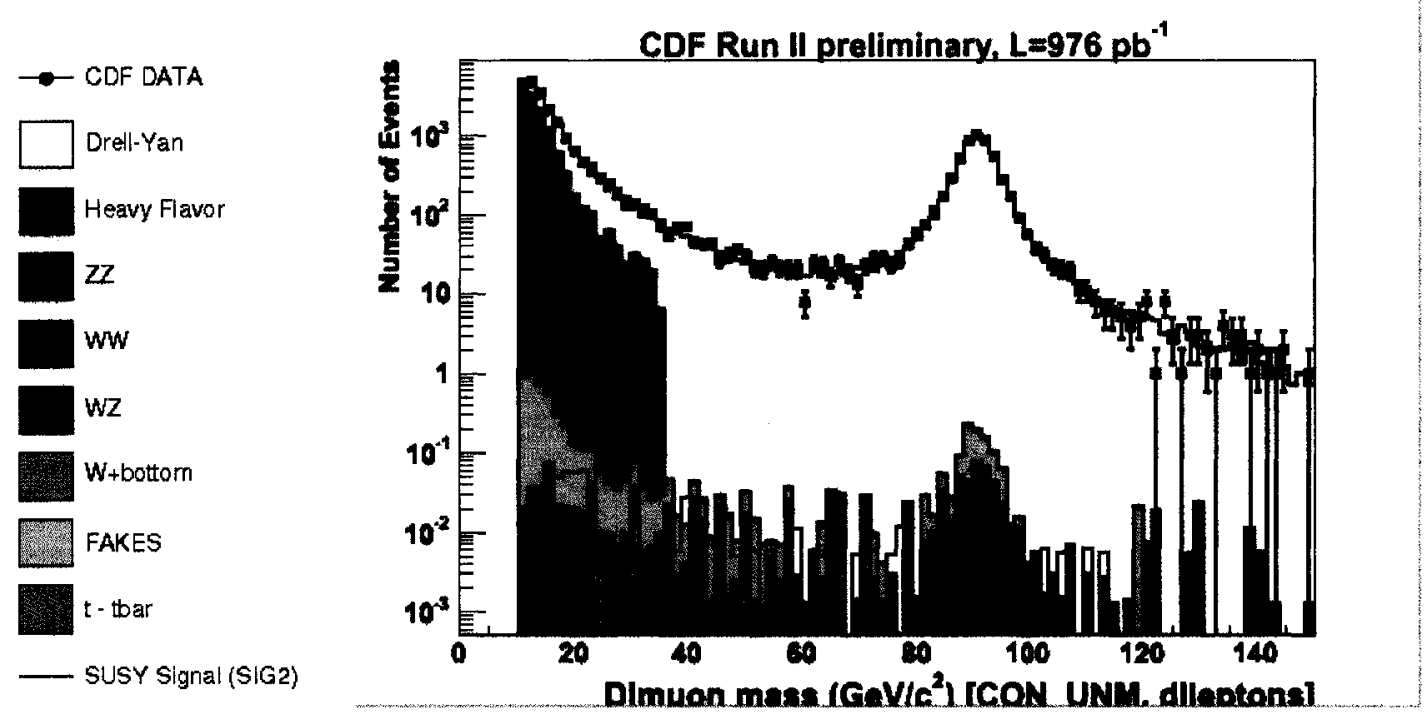

Figure 8.5: Dimuon mass for data, SM prediction, and SUSY MC signal in region CON_UNM 
Chapter 8. Trilepton Analysis
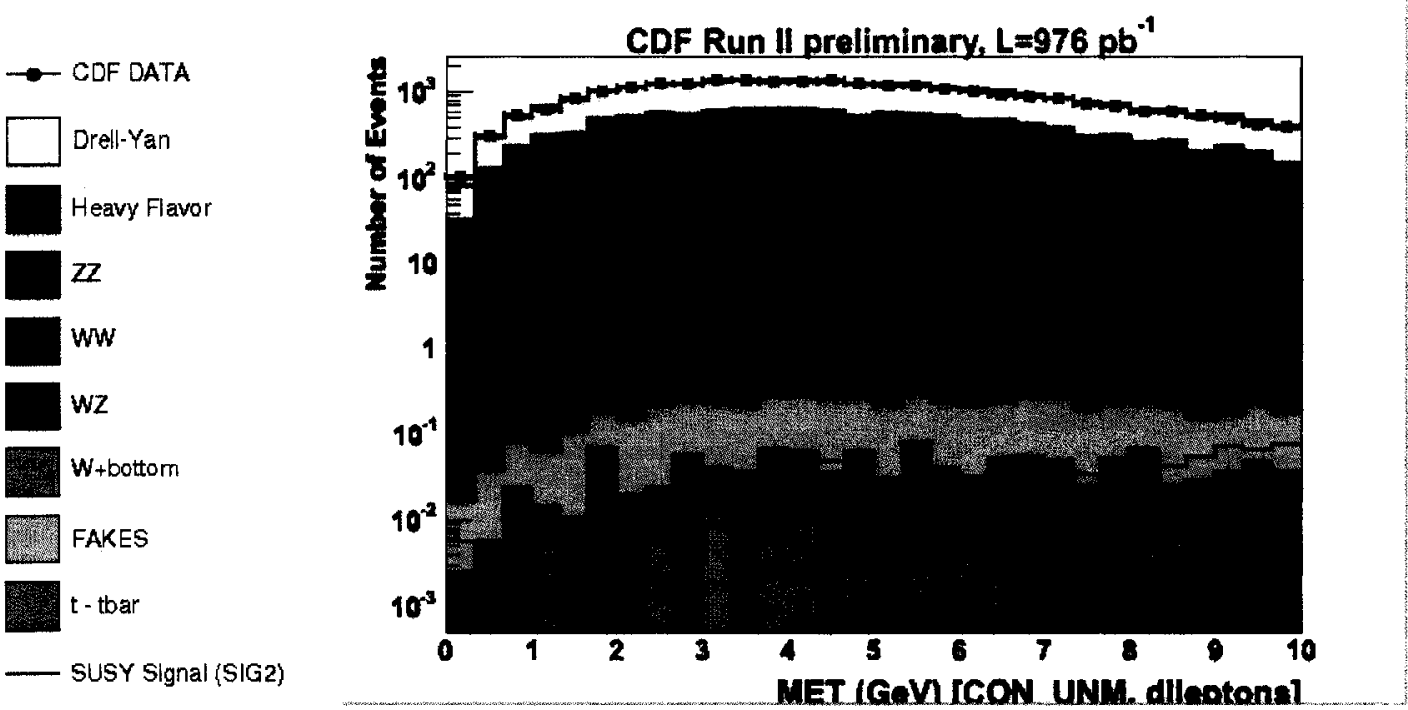

Figure 8.6: $\mathbb{E}_{\mathrm{T}}$ for data, SM prediction, and SUSY MC signal in region CON_UNM
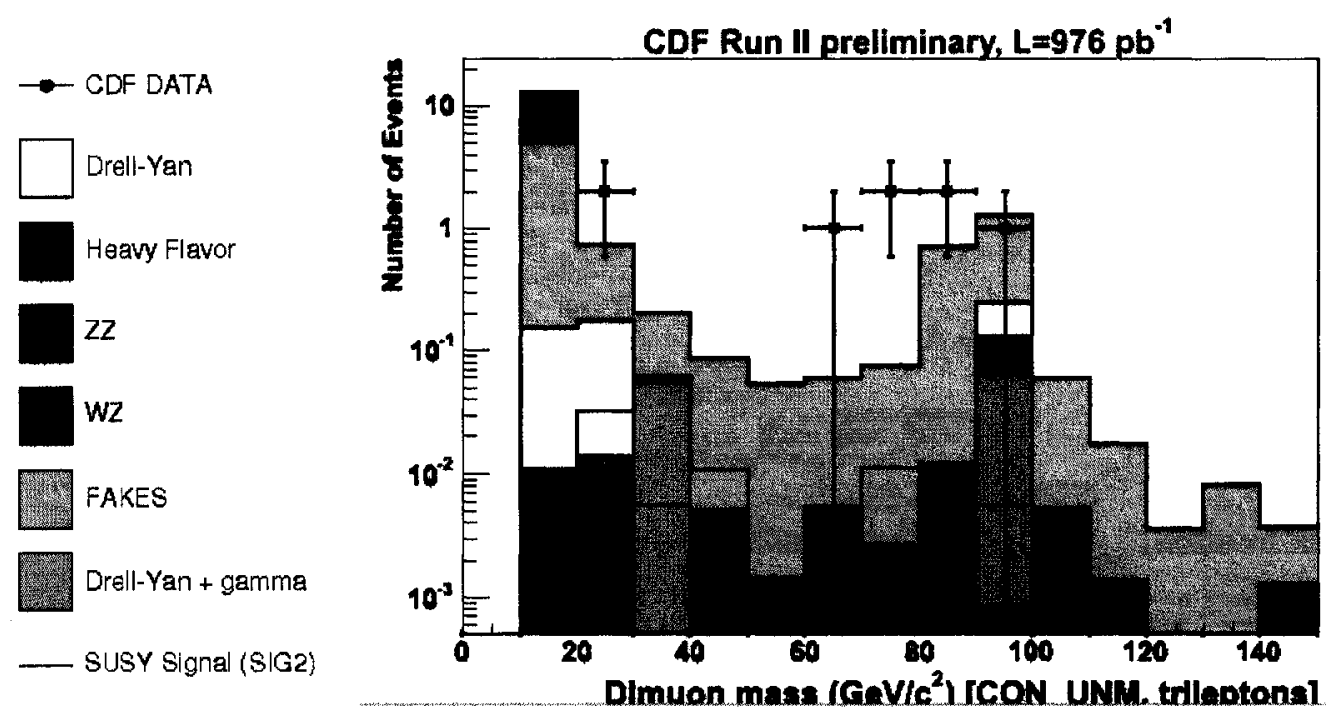

Figure 8.7: Dimuon mass for data, SM prediction, and SUSY MC signal in region CON_UNM 
Chapter 8. Trilepton Analysis
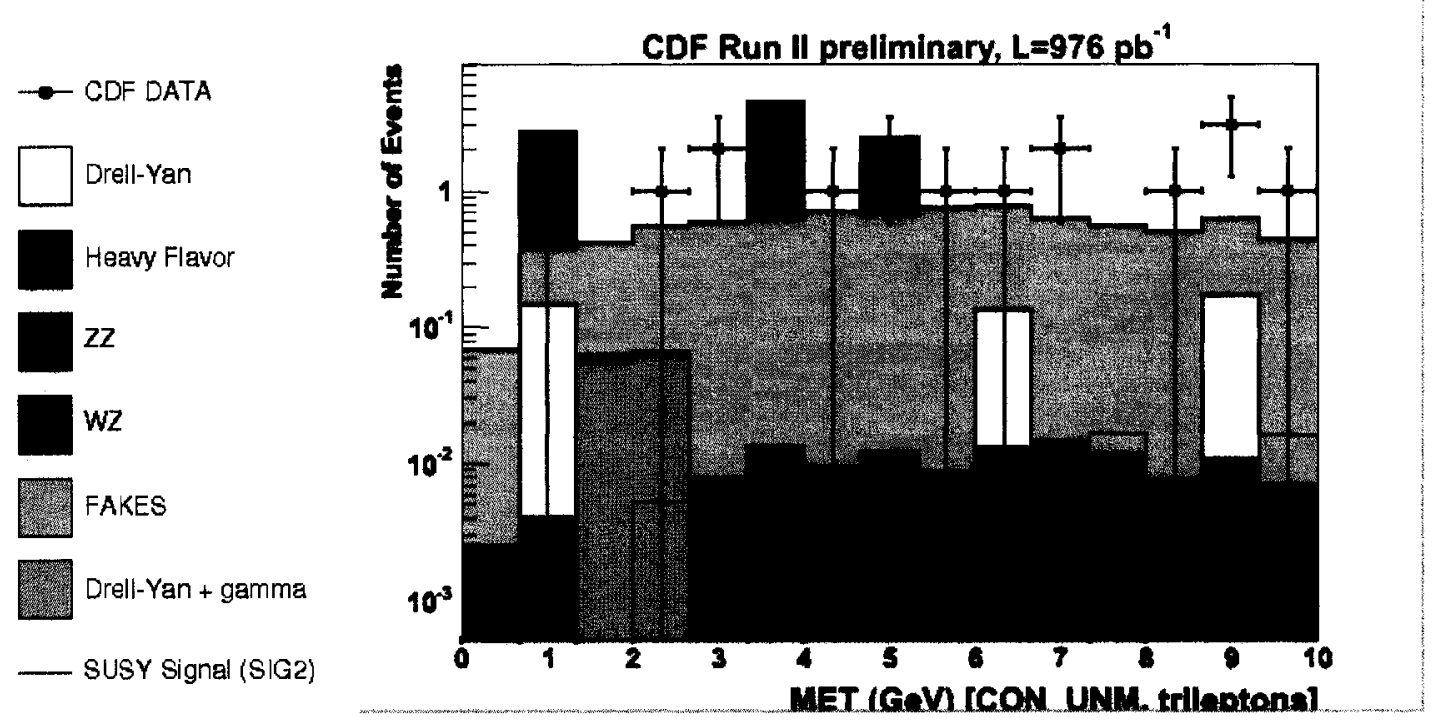

Figure 8.8: $E_{\mathrm{T}}$ for data, SM prediction, and SUSY MC signal in region CON_UNM 
Chapter 8. Trilepton Analysis

\subsubsection{Control Region CON_E}

In this region, we require $\mathbb{E}_{\mathrm{T}}<15 \mathrm{GeV}$, dimuon mass within $Z$ region and low jet multiplicity (only one jet allowed). Figures 8.9 to 8.12 show the $M_{\mu \mu}$ and $\mathbb{E}_{\mathrm{T}}$ distributions for data, SM predicted BG, and SUSY signal for dilepton and trilepton case.
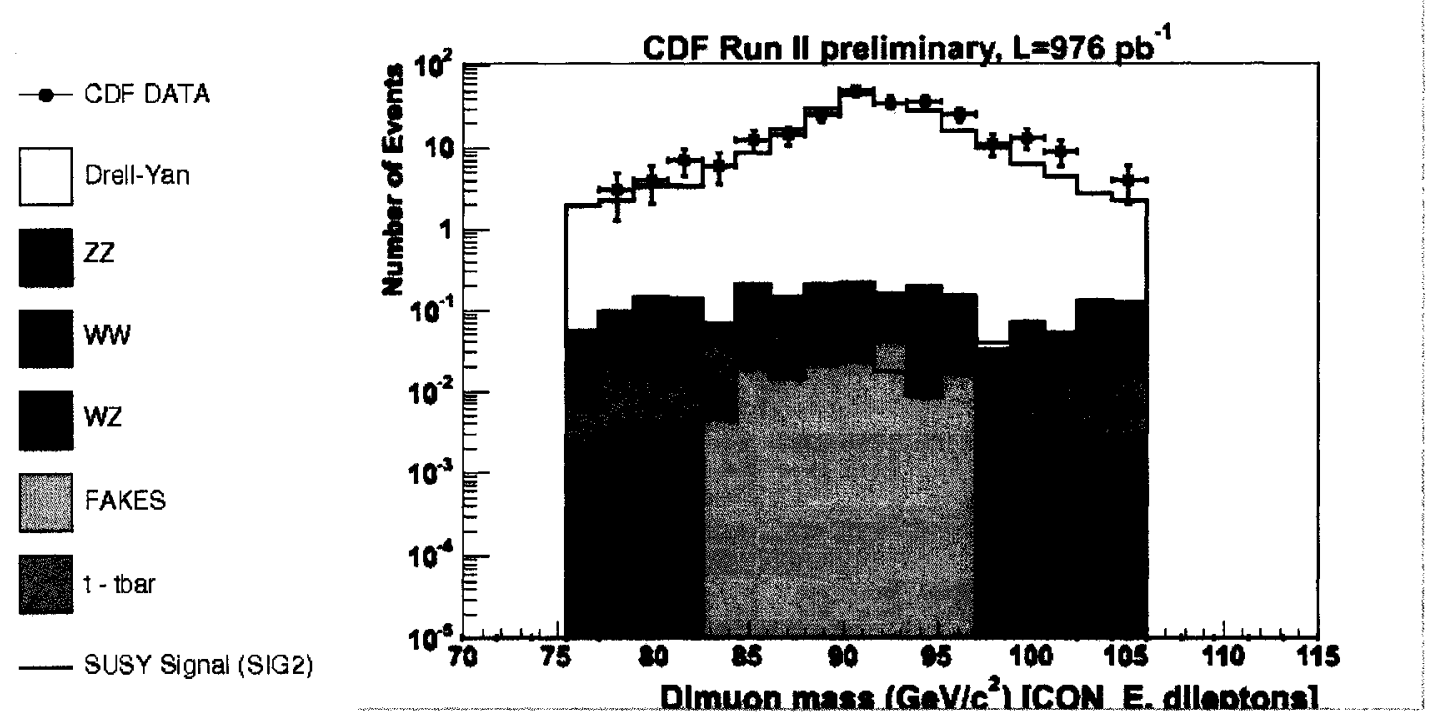

Figure 8.9: Dimuon mass for data, SM prediction, and SUSY MC signal in region CON_E 
Chapter 8. Trilepton Analysis
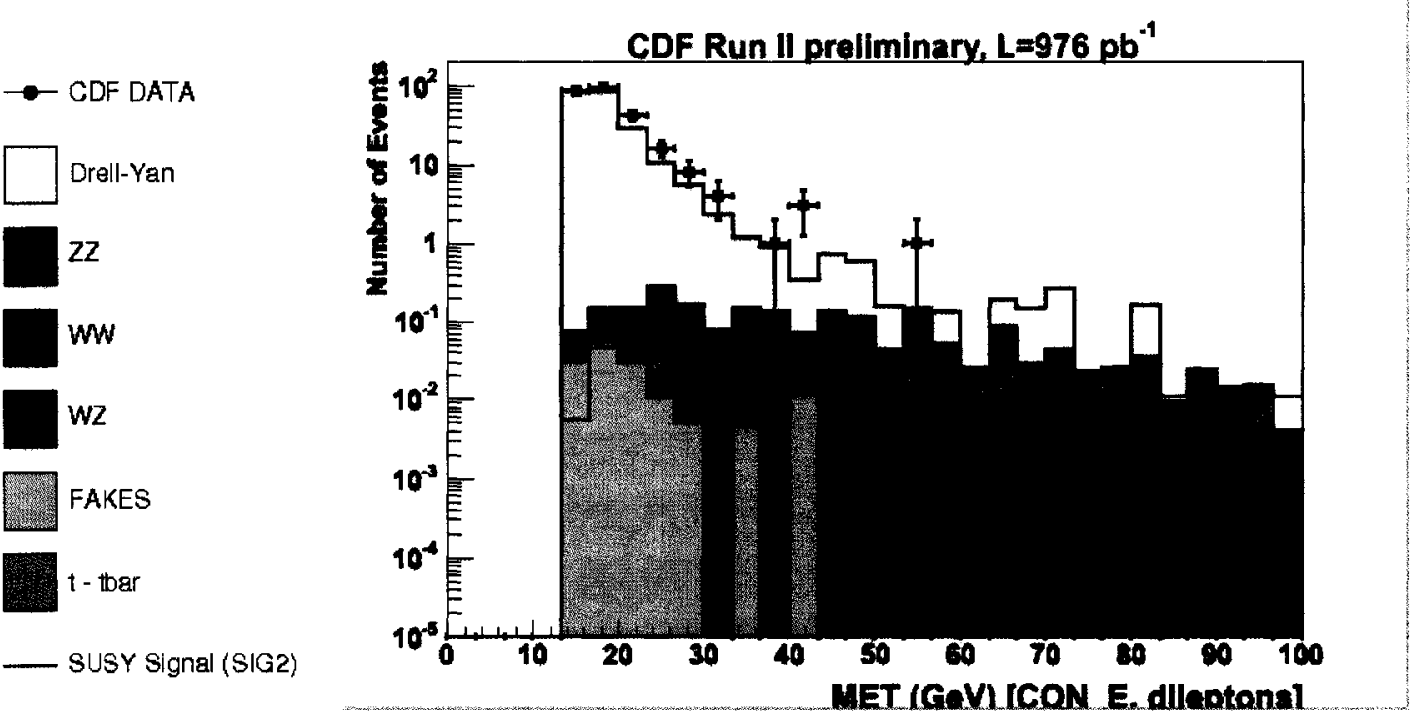

Figure 8.10: $E_{\mathrm{T}}$ for data, SM prediction, and SUSY MC signal in region CON_E
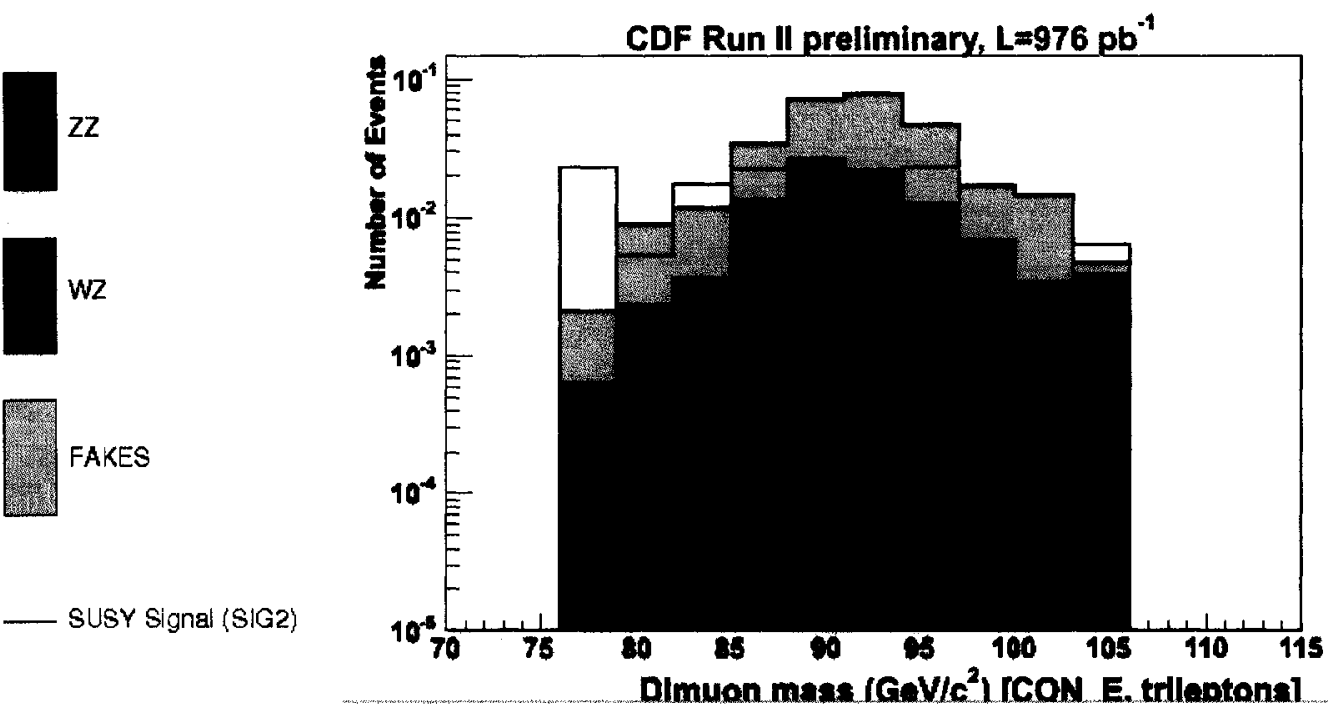

Figure 8.11: Dimuon mass for data, SM prediction, and SUSY MC signal in region CON_E 
Chapter 8. Trilepton Analysis
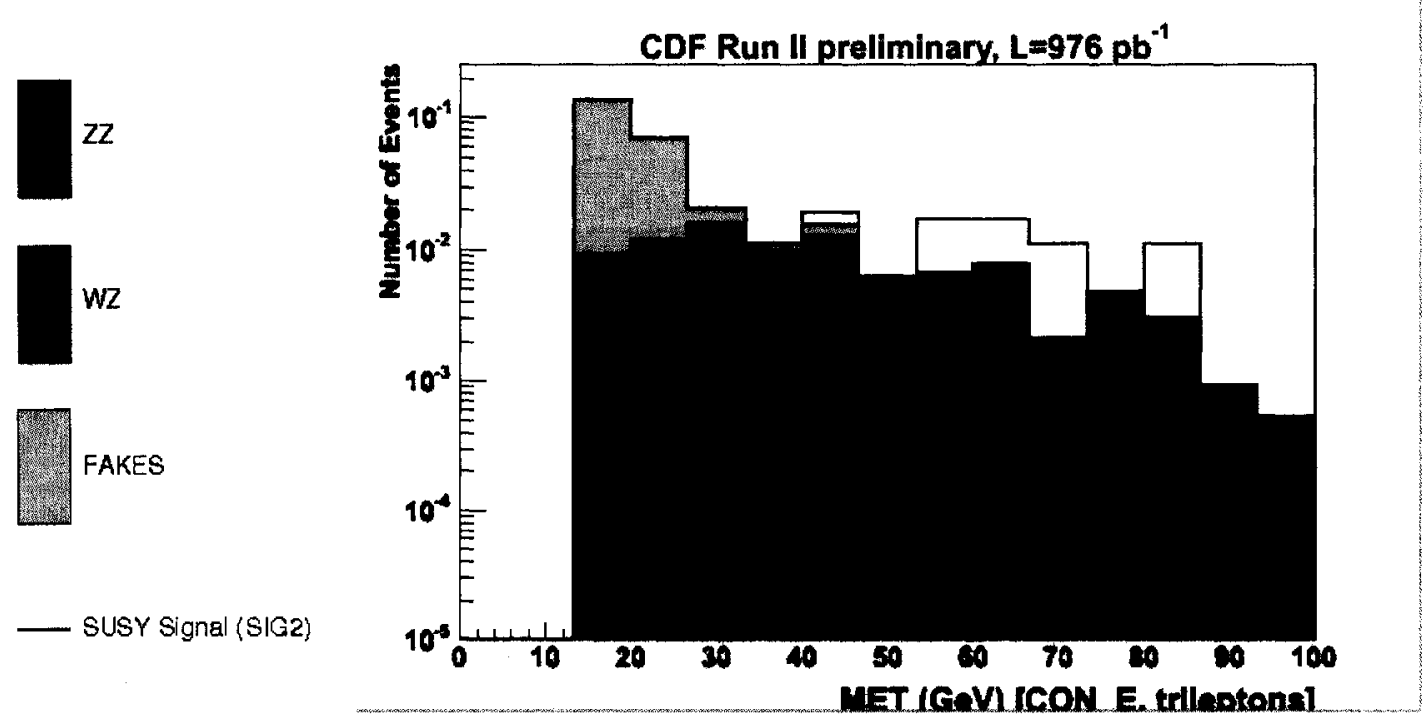

Figure 8.12: $E_{T}$ for data, SM prediction, and SUSY MC signal in region CON_E 
Chapter 8. Trilepton Analysis

\subsubsection{Control Region CON_F}

In this region, we require $\mathrm{E}_{\mathrm{T}}<15 \mathrm{GeV}$, dimuon mass within the $Z$ region and high jet multiplicity (more than one jet). Figures 8.13 to 8.16 show the $M_{\mu \mu}$ and $E_{\mathrm{T}}$ distributions for data, SM predicted BG, and SUSY signal for dilepton and trilepton case.
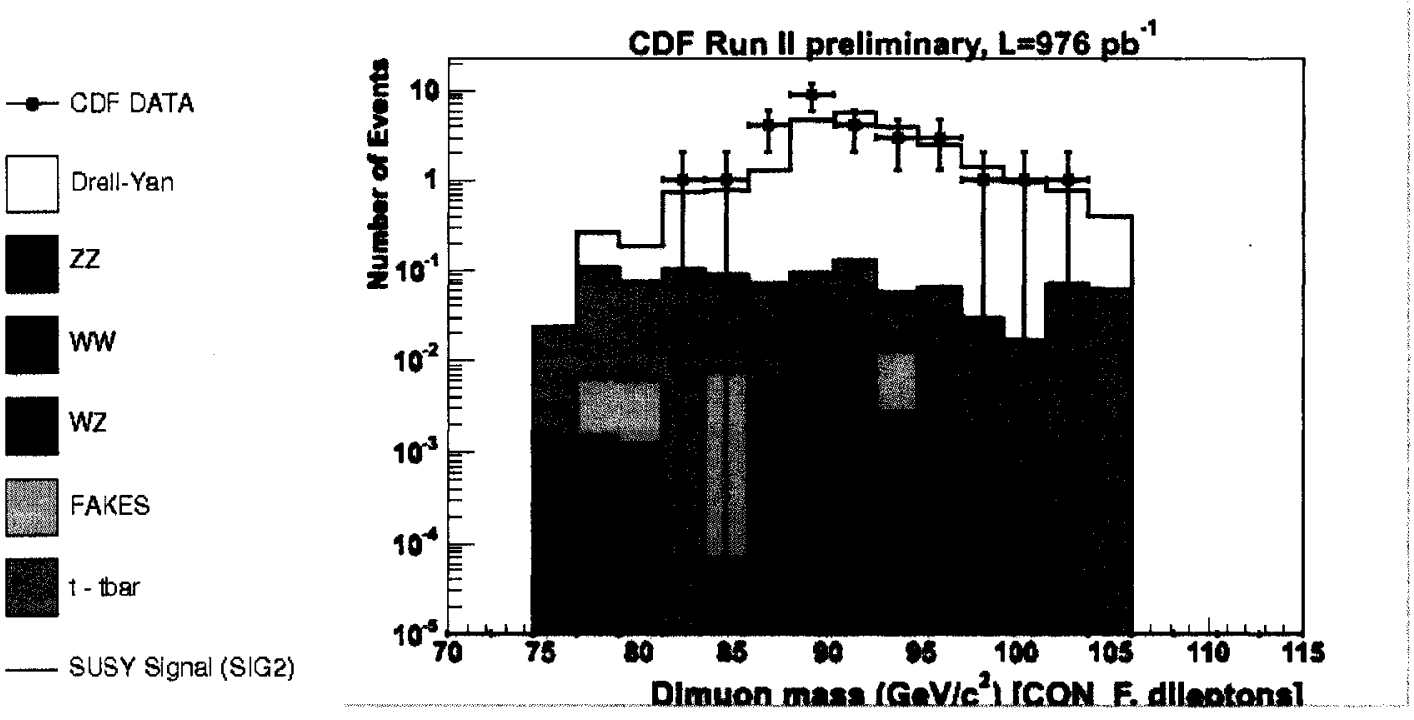

Figure 8.13: Dimuon mass for data, SM prediction, and SUSY MC signal in region CON_F 
Chapter 8. Trilepton Analysis
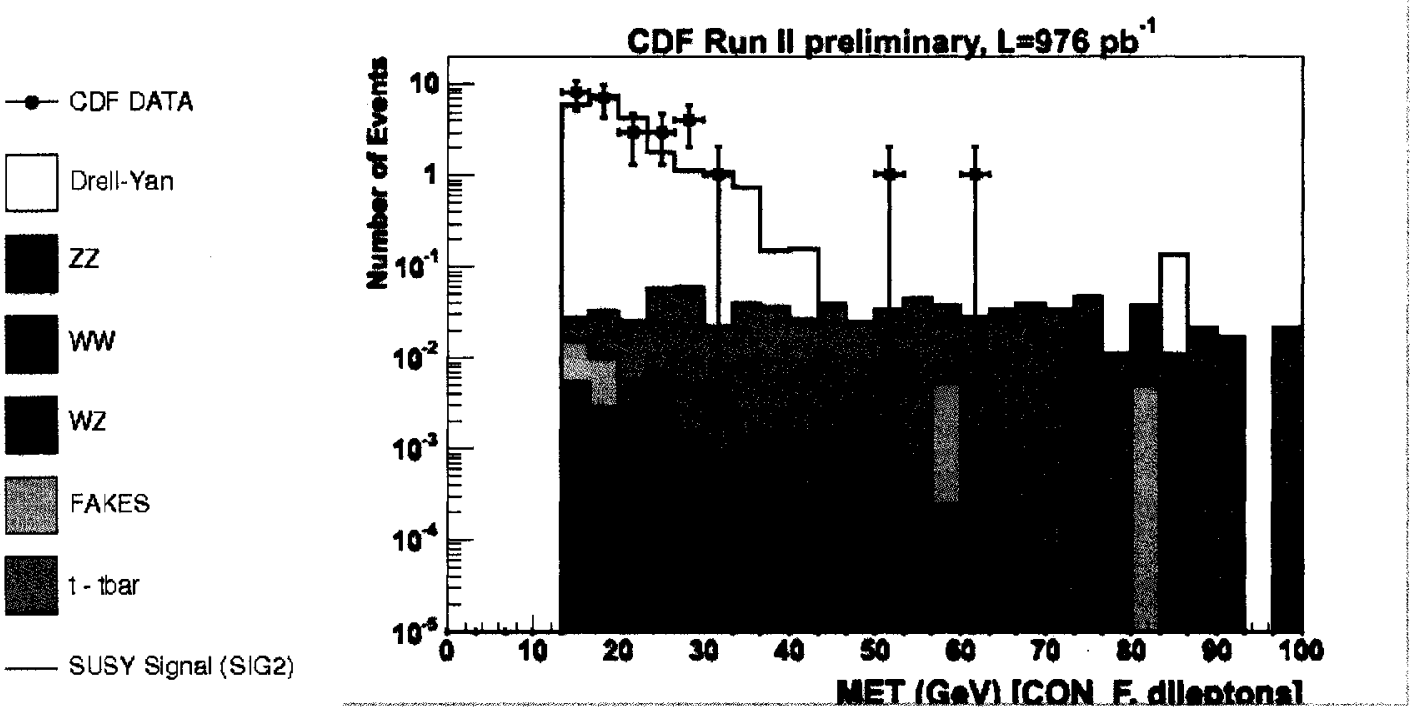

Figure 8.14: $E_{\mathrm{T}}$ for data, SM prediction, and SUSY MC signal in region CON_F
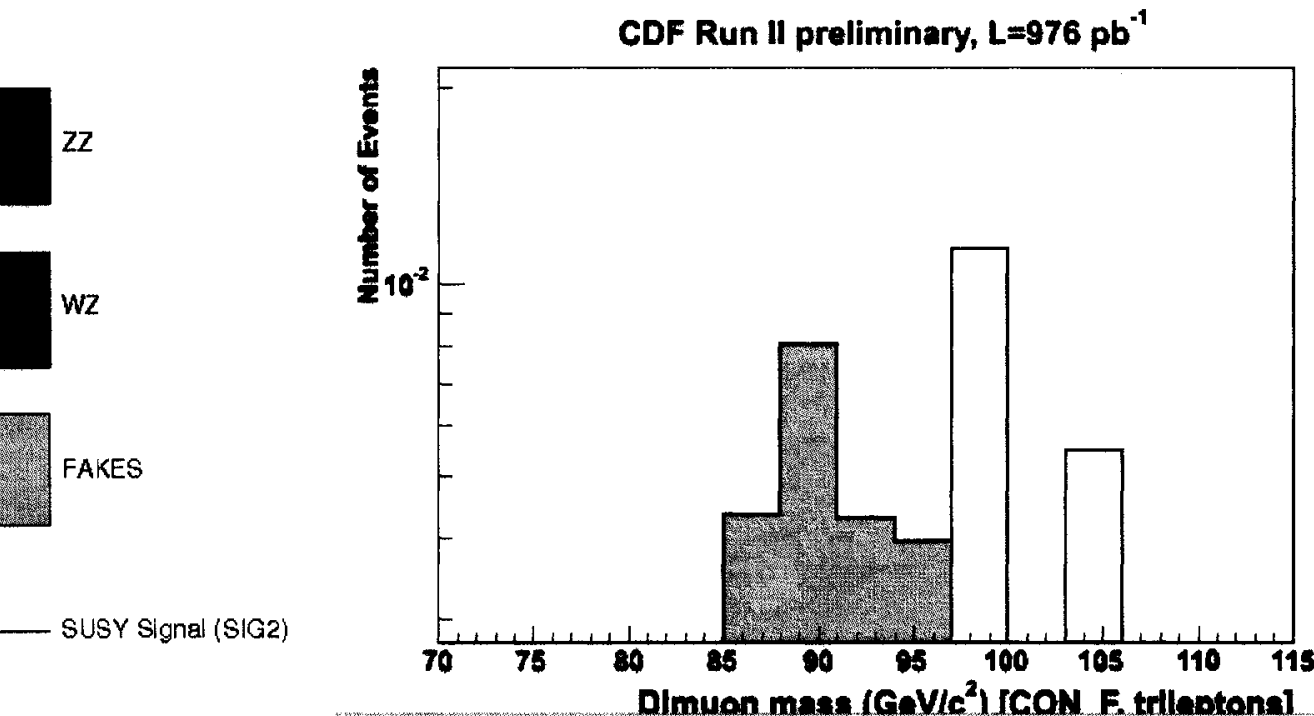

Figure 8.15: Dimuon mass for data, SM prediction, and SUSY MC signal in region CON_F 
Chapter 8. Trilepton Analysis

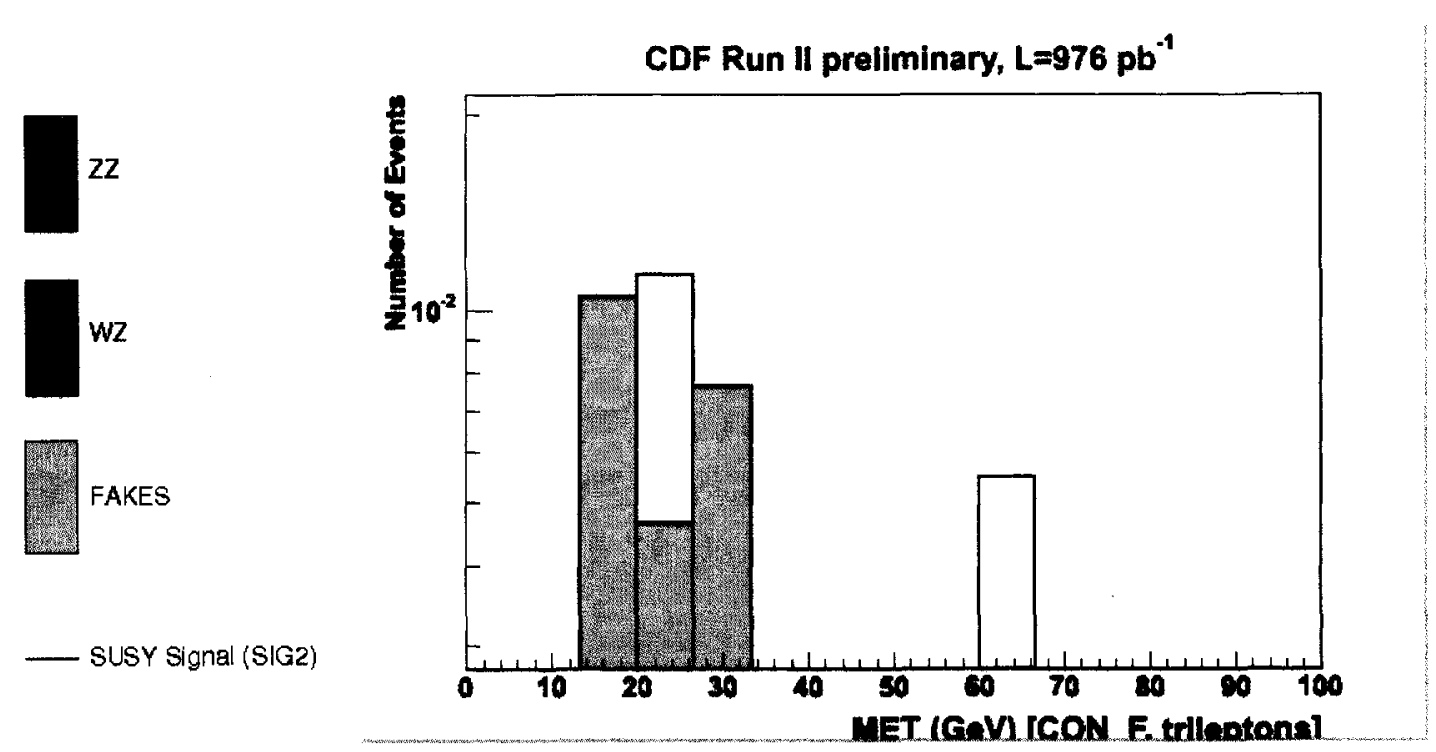

Figure 8.16: $E_{\mathrm{T}}$ for data, SM prediction, and SUSY MC signal in region CON_F 
Chapter 8. Trilepton Analysis

\subsubsection{Control Region CON_G}

In this region, we only require $E_{\mathrm{T}}<10 \mathrm{GeV}$. Figures 8.17 to 8.20 show the $M_{\mu \mu}$ and $\mathbb{E}_{\mathrm{T}}$ distributions for data, SM predicted BG, and SUSY signal for dilepton and trilepton case.
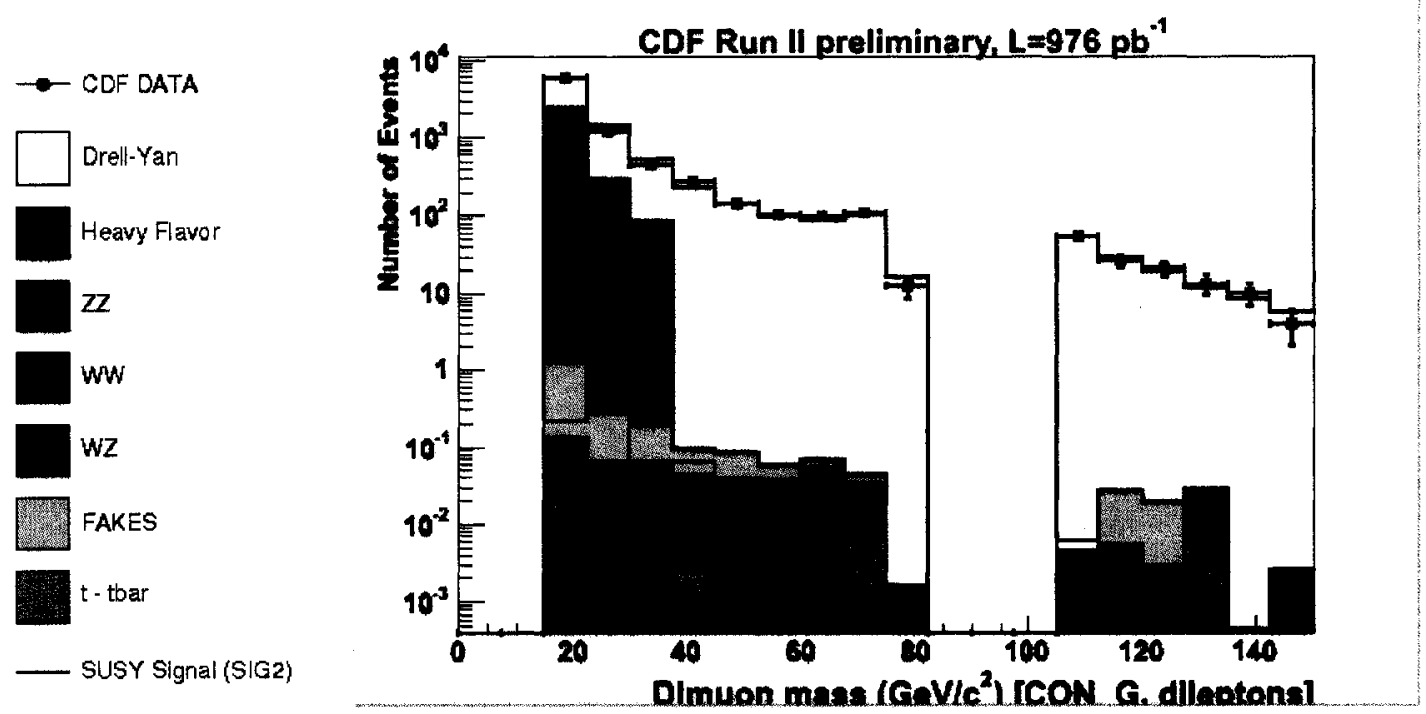

Figure 8.17: Dimuon mass for data, SM prediction, and SUSY MC signal in region CON_G 
Chapter 8. Trilepton Analysis
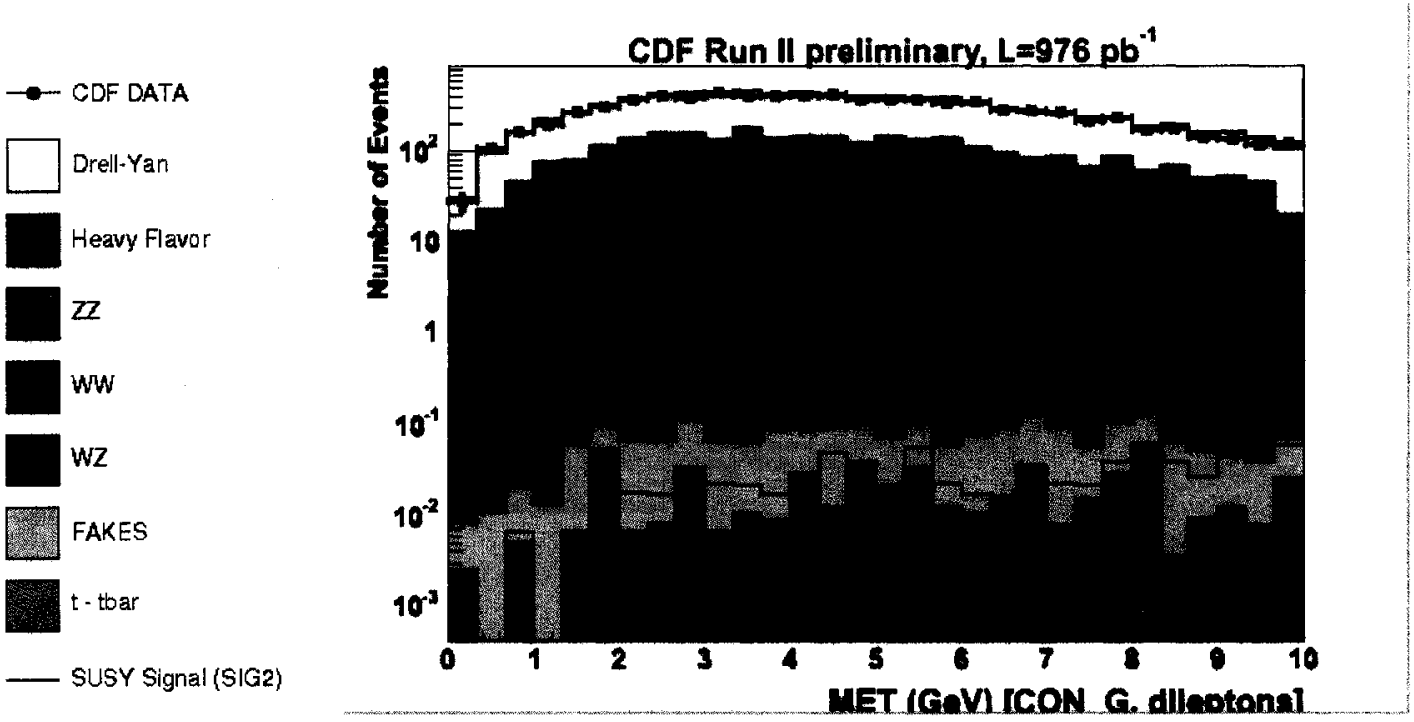

Figure 8.18: $E_{\mathrm{T}}$ for data, SM prediction, and SUSY MC signal in region CON_G
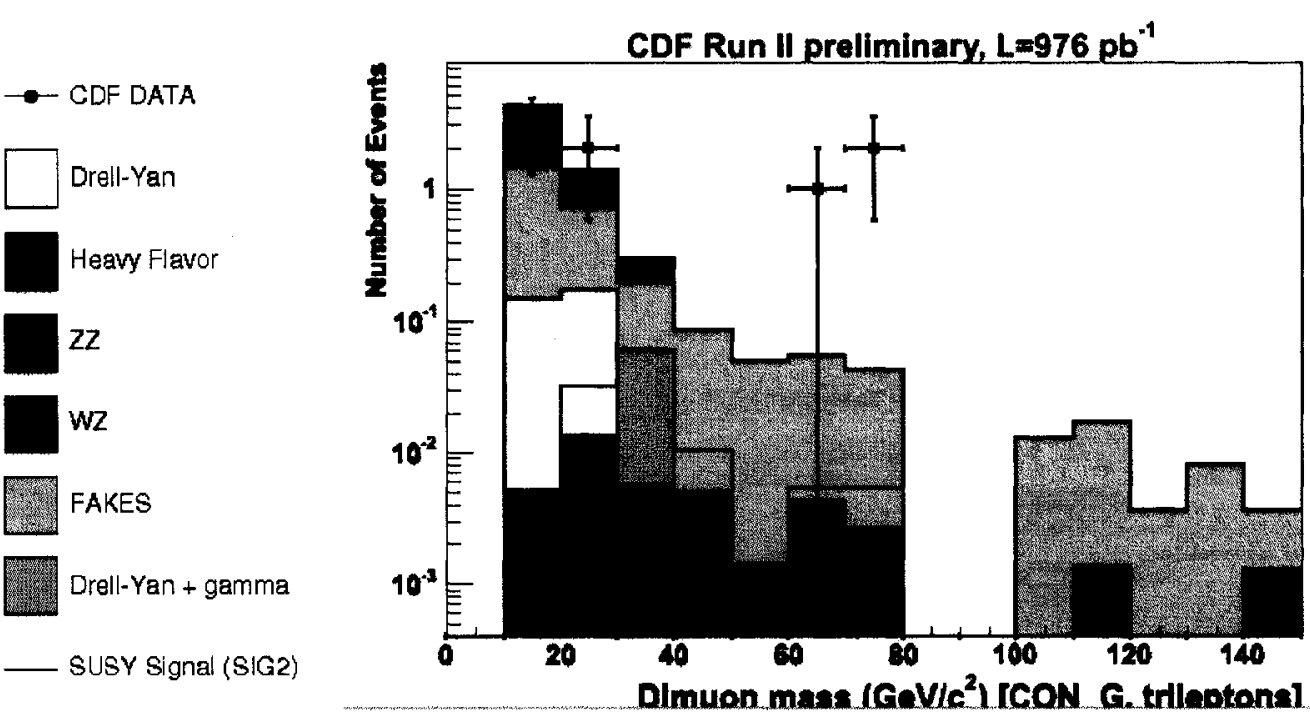

Figure 8.19: Dimuon mass for data, SM prediction, and SUSY MC signal in region CON_G 
Chapter 8. Trilepton Analysis
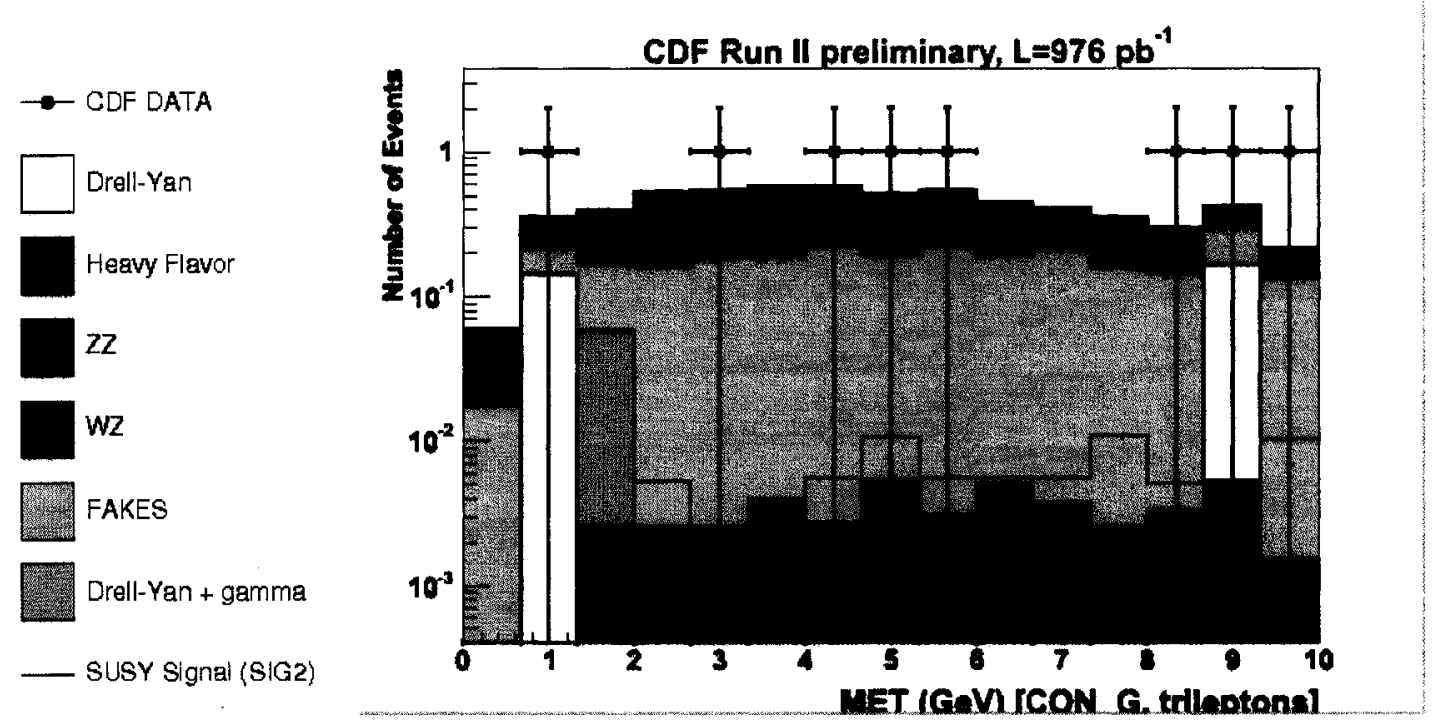

Figure 8.20: $\mathbb{E}_{\mathrm{T}}$ for data, SM prediction, and SUSY MC signal in region CON_G 
Chapter 8. Trilepton Analysis

\subsubsection{Control Region CON_H}

In this region, we only require $E_{\mathrm{T}}<10 \mathrm{GeV}$. Figures 8.21 to 8.24 show the $M_{\mu \mu}$ and $E_{\mathrm{T}}$ distributions for data, SM predicted BG, and SUSY signal for dilepton and trilepton case.

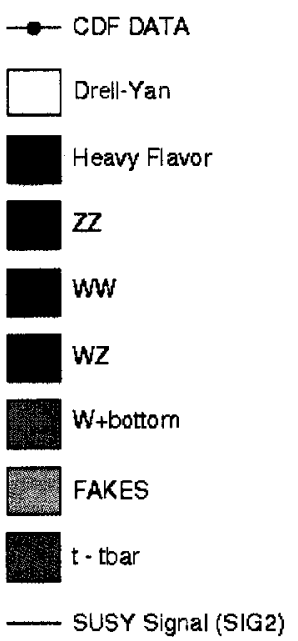

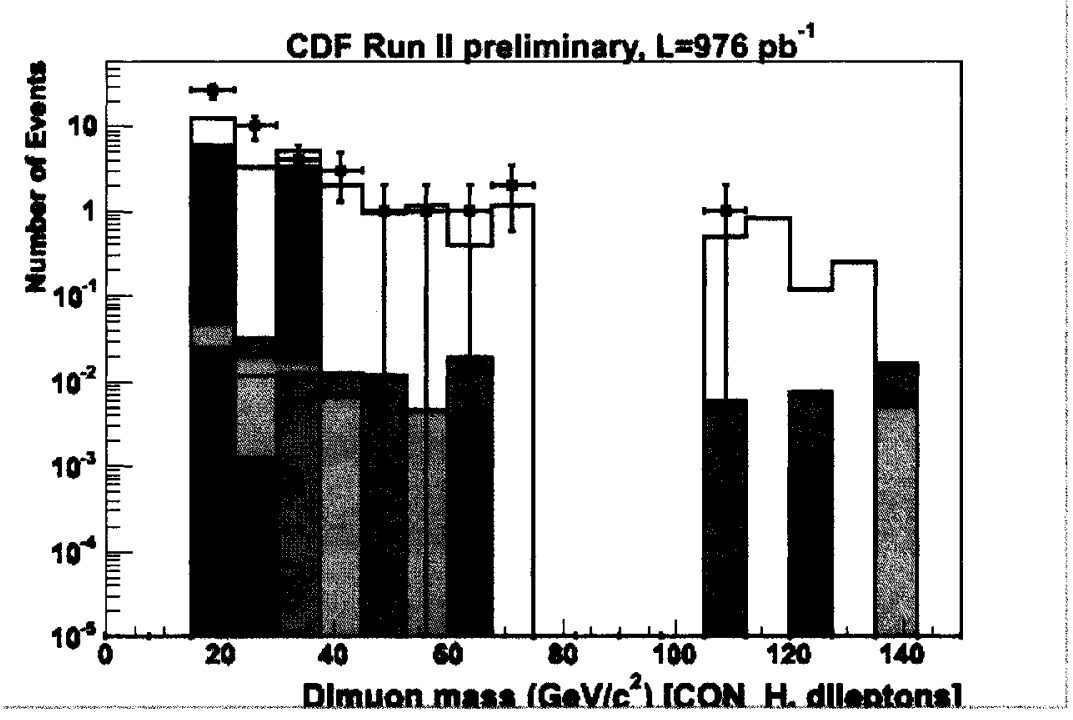

Figure 8.21: Dimuon mass for data, SM prediction, and SUSY MC signal in region CON_H 
Chapter 8. Trilepton Analysis
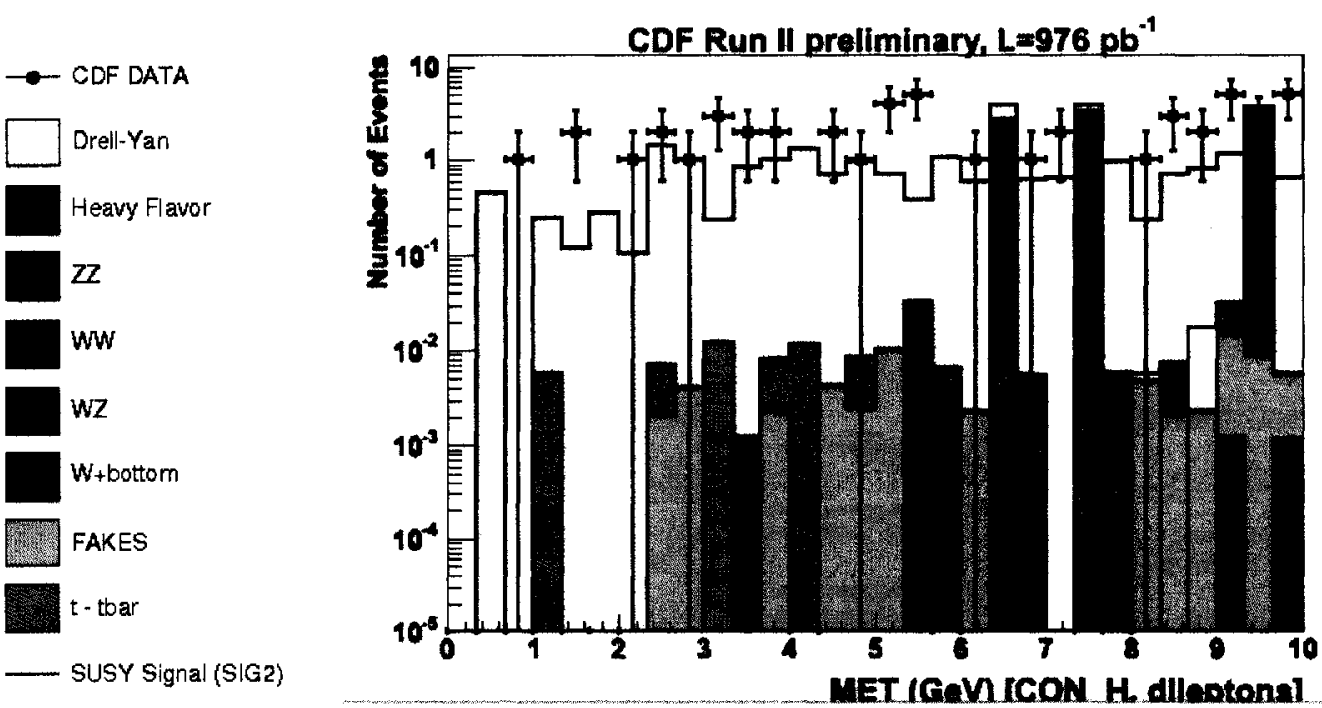

Figure 8.22: $E_{\mathrm{T}}$ for data, SM prediction, and SUSY MC signal in region CON_H
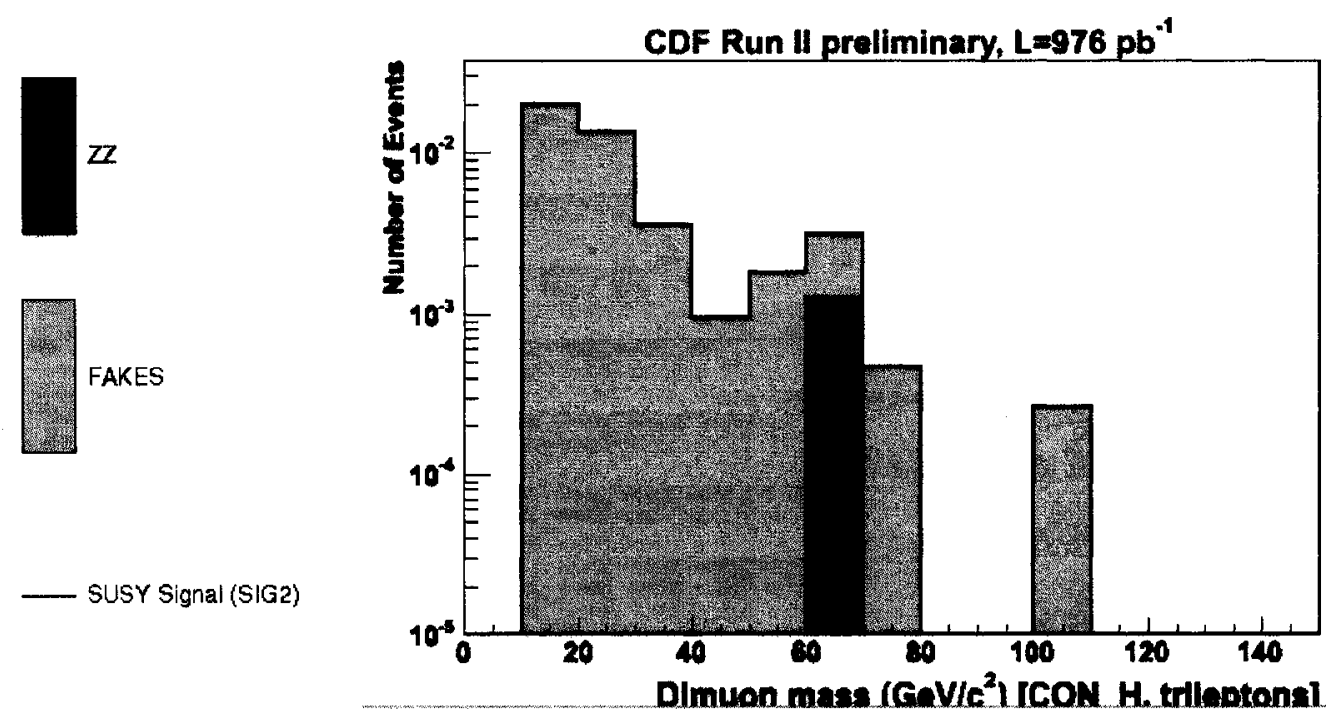

Figure 8.23: Dimuon mass for data, SM prediction, and SUSY MC signal in region CON_H 
Chapter 8. Trilepton Analysis
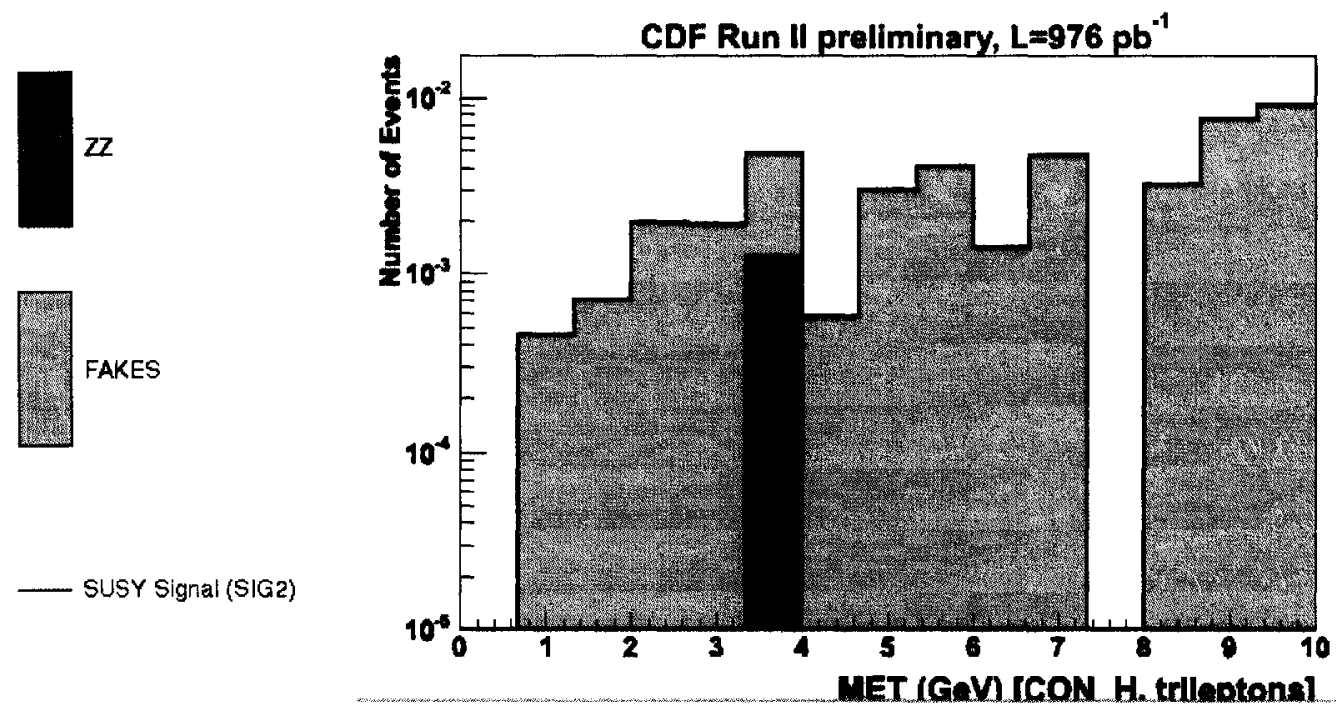

Figure 8.24: $\mathbb{E}_{\mathrm{T}}$ for data, SM prediction, and SUSY MC signal in region CON_H 
Chapter 8. Trilepton Analysis

\subsubsection{Control Region CON_I}

In this region, we only require $\mathbb{E}_{\mathrm{T}}<10 \mathrm{GeV}$. Figures 8.25 to 8.28 show the $M_{\mu \mu}$ and $E_{\mathrm{T}}$ distributions for data, SM predicted BG, and SUSY signal for dilepton and trilepton case.
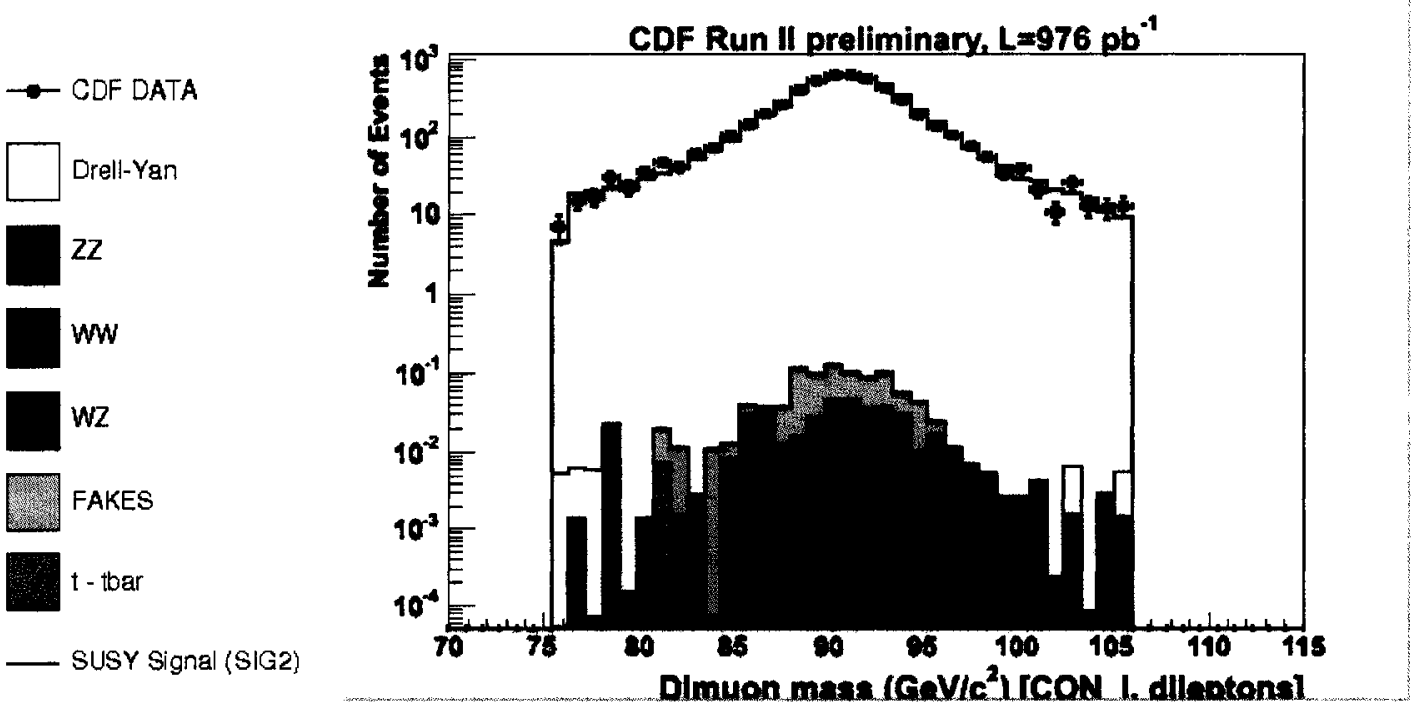

Figure 8.25: Dimuon mass for data, SM prediction, and SUSY MC signal in region CON_I 
Chapter 8. Trilepton Analysis
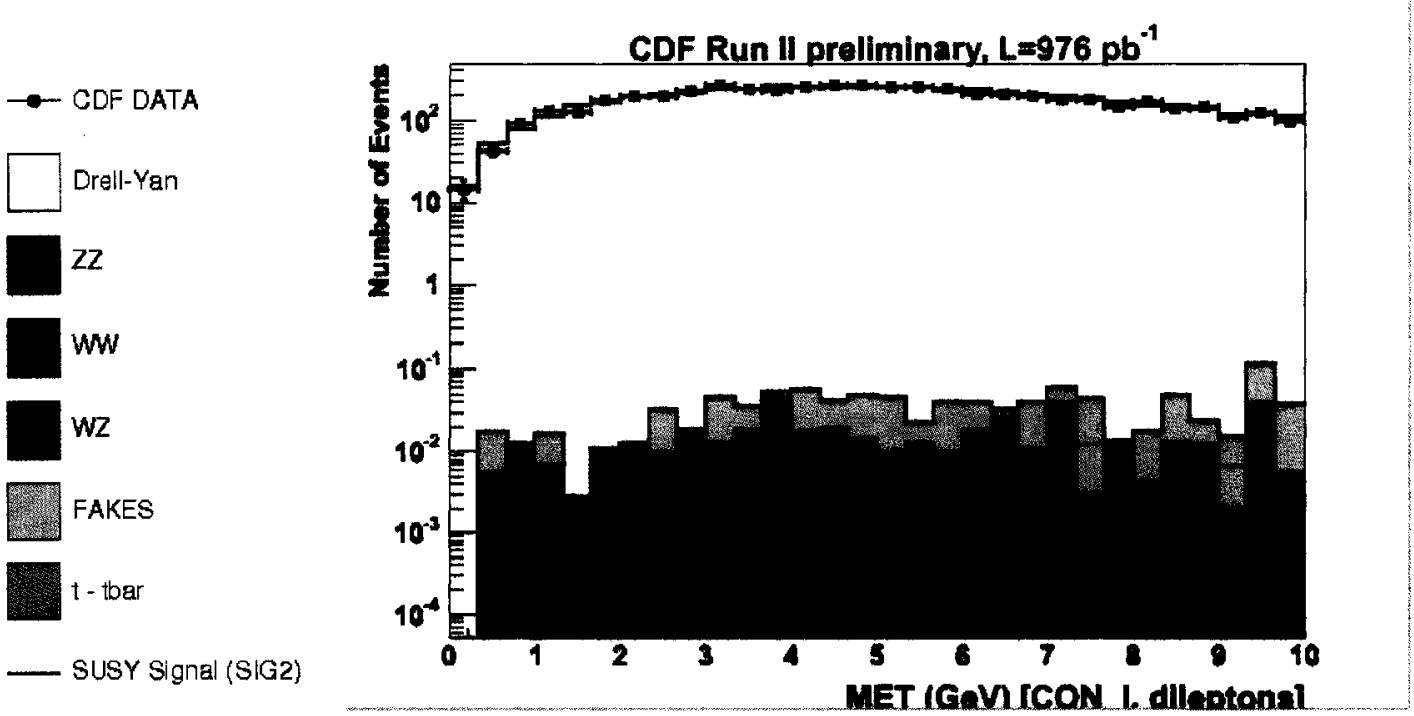

Figure 8.26: $E_{\mathrm{T}}$ for data, SM prediction, and SUSY MC signal in region CON_I
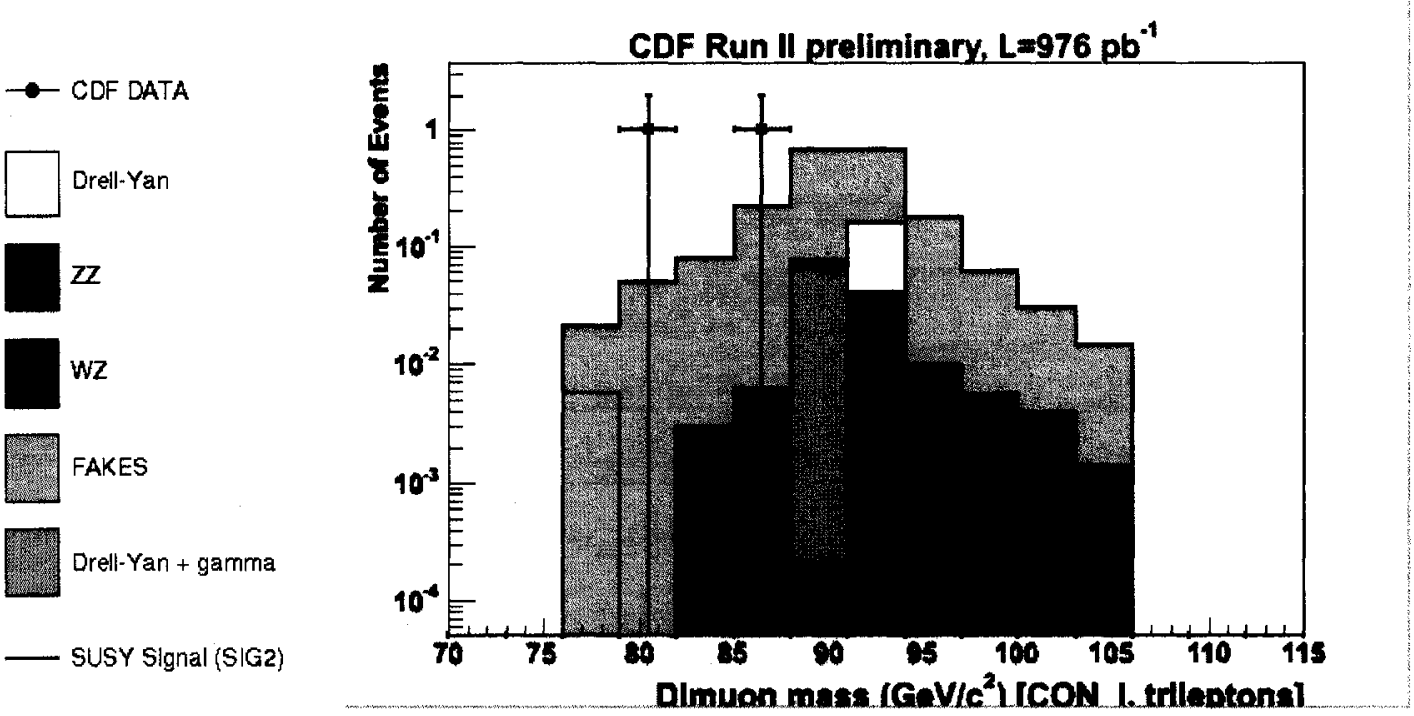

Figure 8.27: Dimuon mass for data, SM prediction, and SUSY MC signal in region CON_I 
Chapter 8. Trilepton Analysis
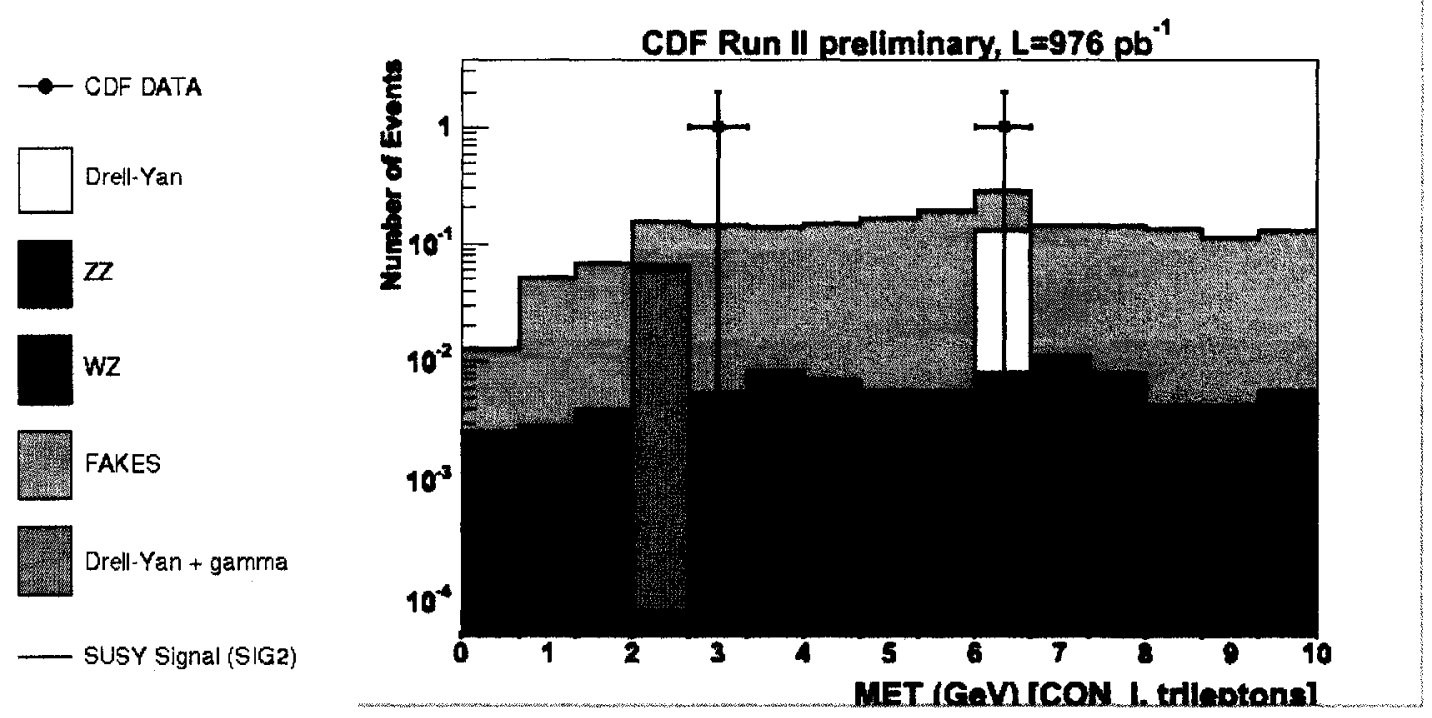

Figure 8.28: $E_{\mathrm{T}}$ for data, SM prediction, and SUSY MC signal in region CON_I 
Chapter 8. Trilepton Analysis

\subsubsection{Control Region CON_J}

In this region, we only require $E_{\mathrm{T}}<10 \mathrm{GeV}$. Figures 8.29 to 8.32 show the $M_{\mu \mu}$ and $E_{\mathrm{T}}$ distributions for data, SM predicted BG, and SUSY signal for dilepton and trilepton case.
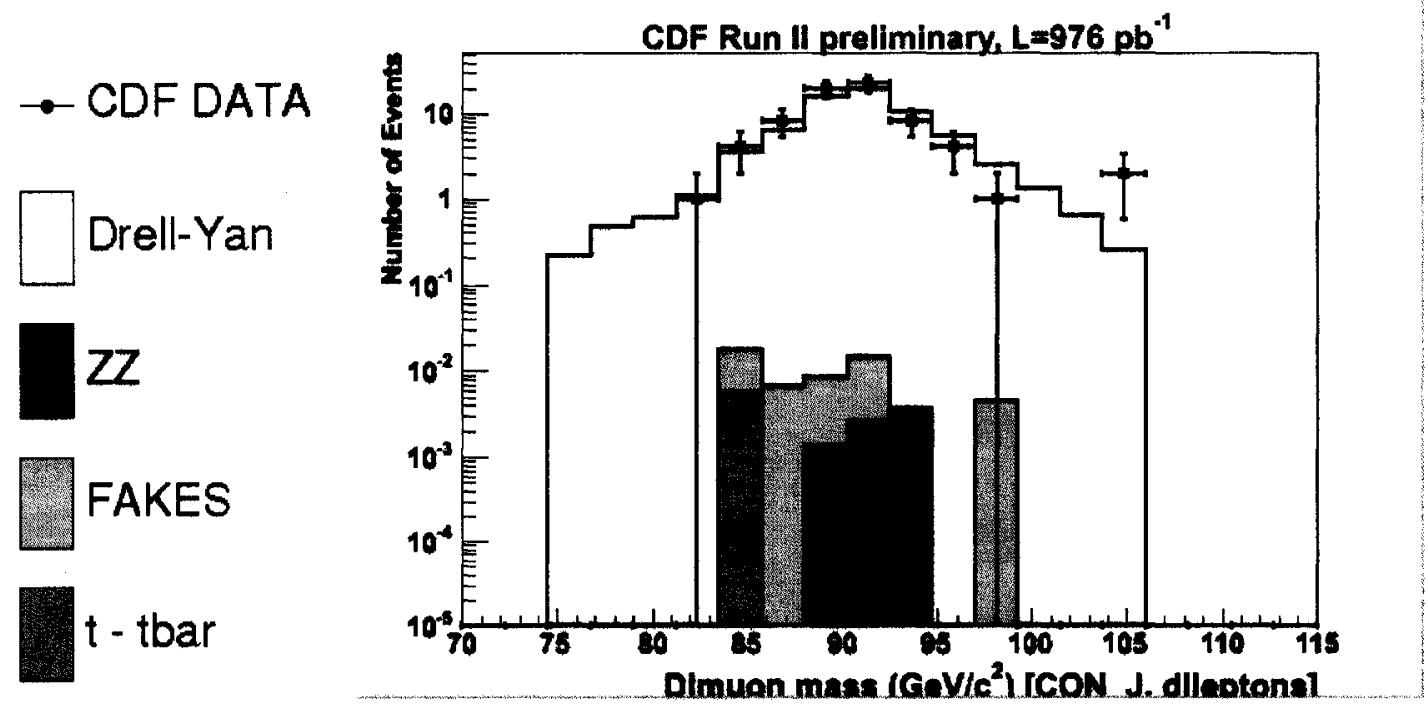

Figure 8.29: Dimuon mass for data, SM prediction, and SUSY MC signal in region CON_J 

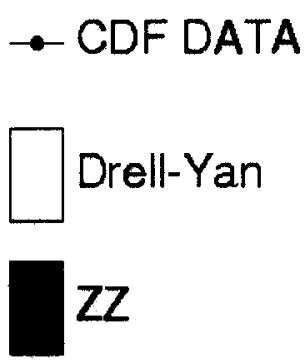

- FAKES

t - tbar

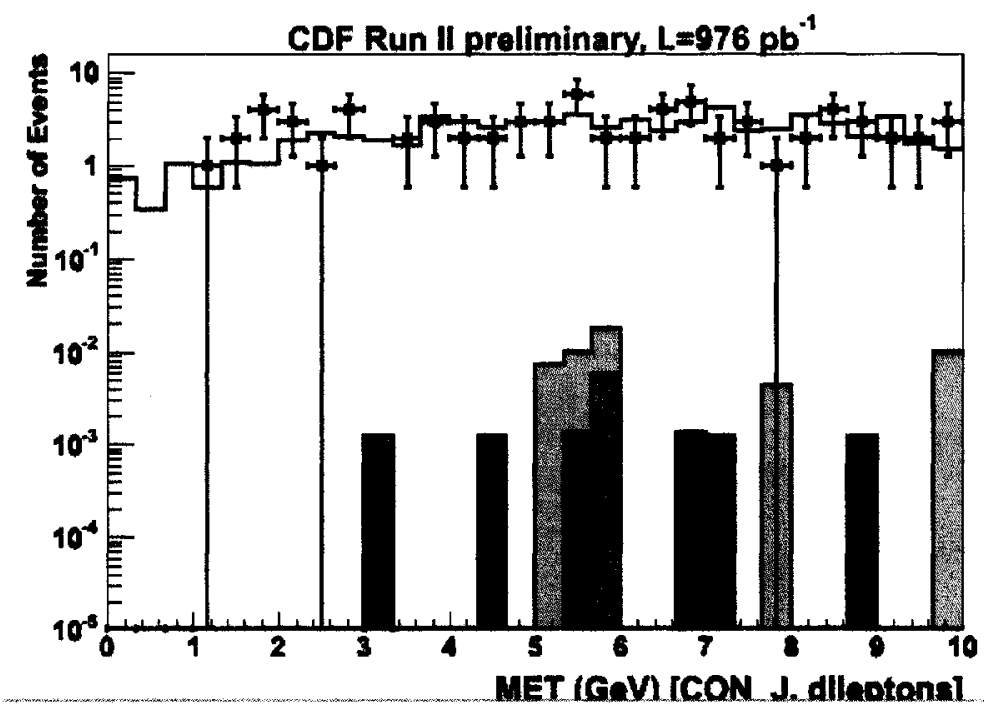

Figure 8.30: $\mathbb{E}_{\mathrm{T}}$ for data, SM prediction, and SUSY MC signal in region CON_J
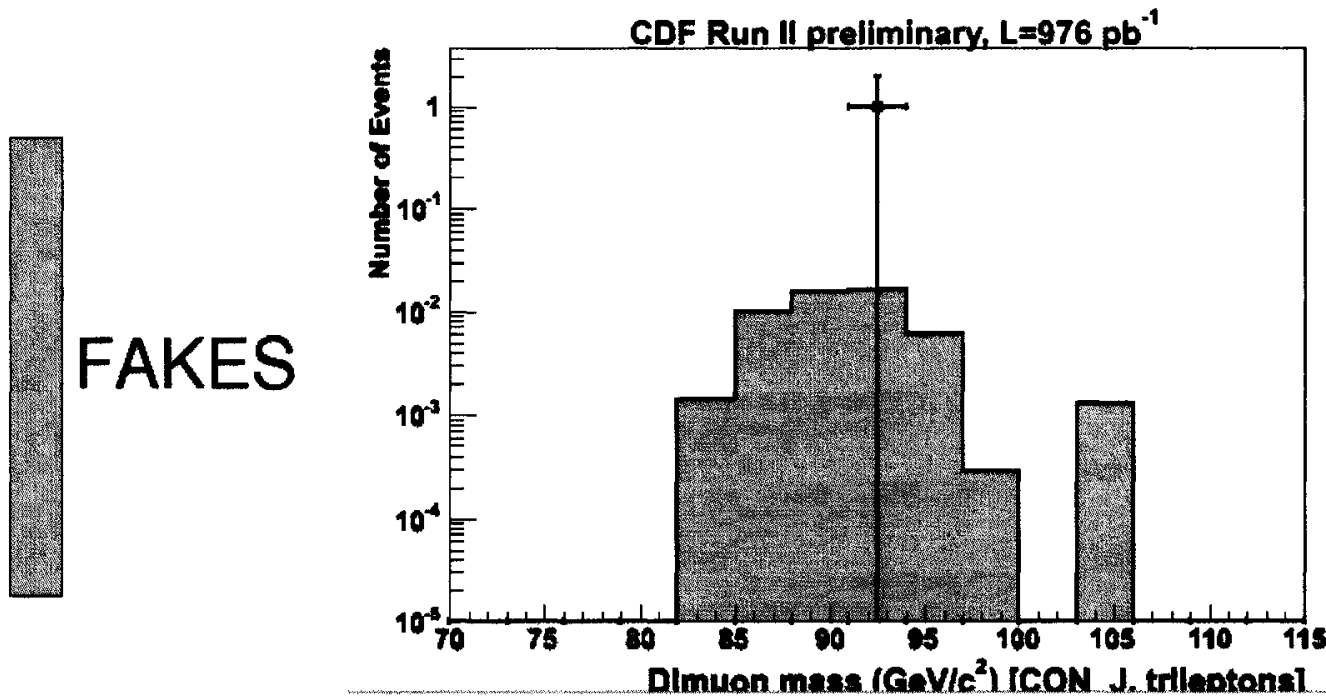

Figure 8.31: Dimuon mass for data, SM prediction, and SUSY MC signal in region CON_J 
Chapter 8. Trilepton Analysis

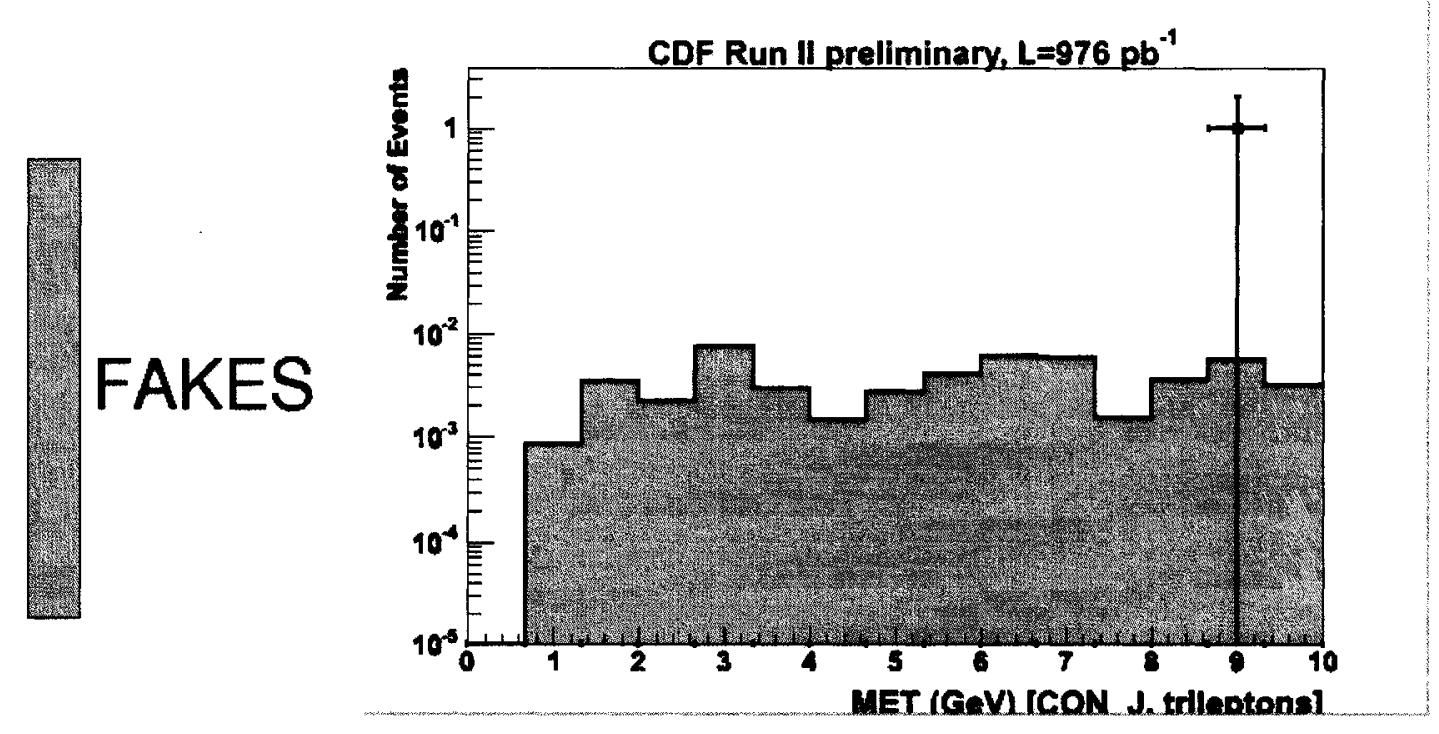

Figure 8.32: $E_{\mathrm{T}}$ for data, SM prediction, and SUSY MC signal in region CON_J 
Chapter 8. Trilepton Analysis

\subsubsection{Control Region SIG_A}

In this region, we only require $\mathbb{E}_{\mathrm{T}}<10 \mathrm{GeV}$. Figures 8.33 to 8.36 show the $M_{\mu \mu}$ and $\mathbb{E}_{\mathrm{T}}$ distributions for data, SM predicted BG, and SUSY signal for dilepton and trilepton case.
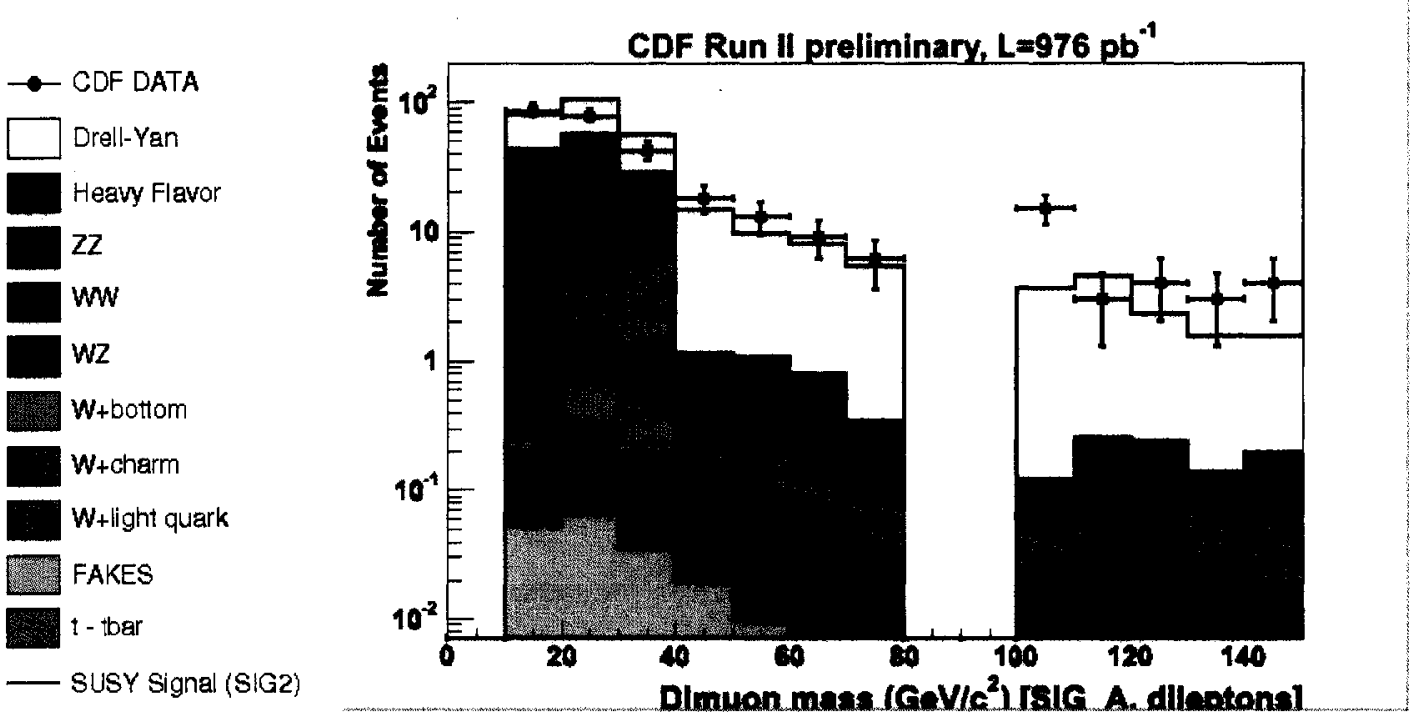

Figure 8.33: Dimuon mass for data, SM prediction, and SUSY MC signal in region SIG_A 
Chapter 8. Trilepton Analysis
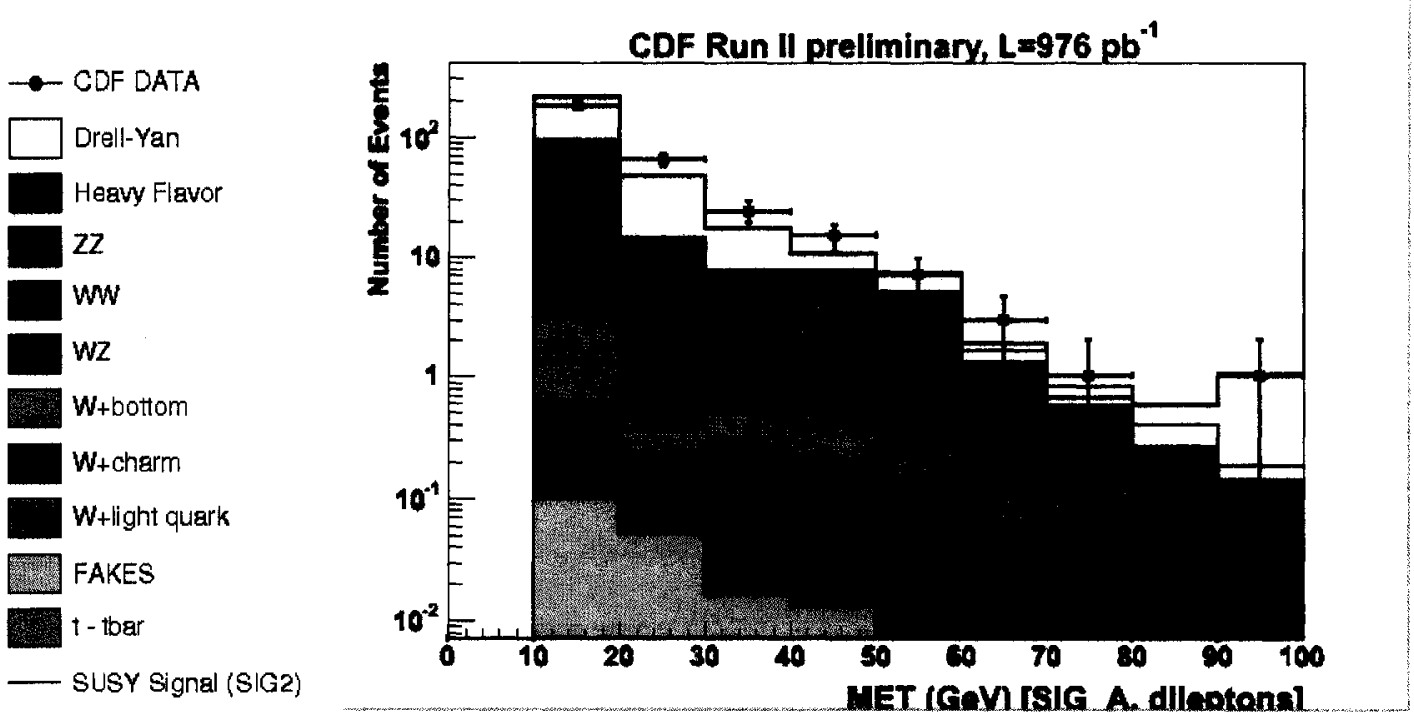

Figure 8.34: $E_{\mathrm{T}}$ for data, SM prediction, and SUSY MC signal in region SIG_A
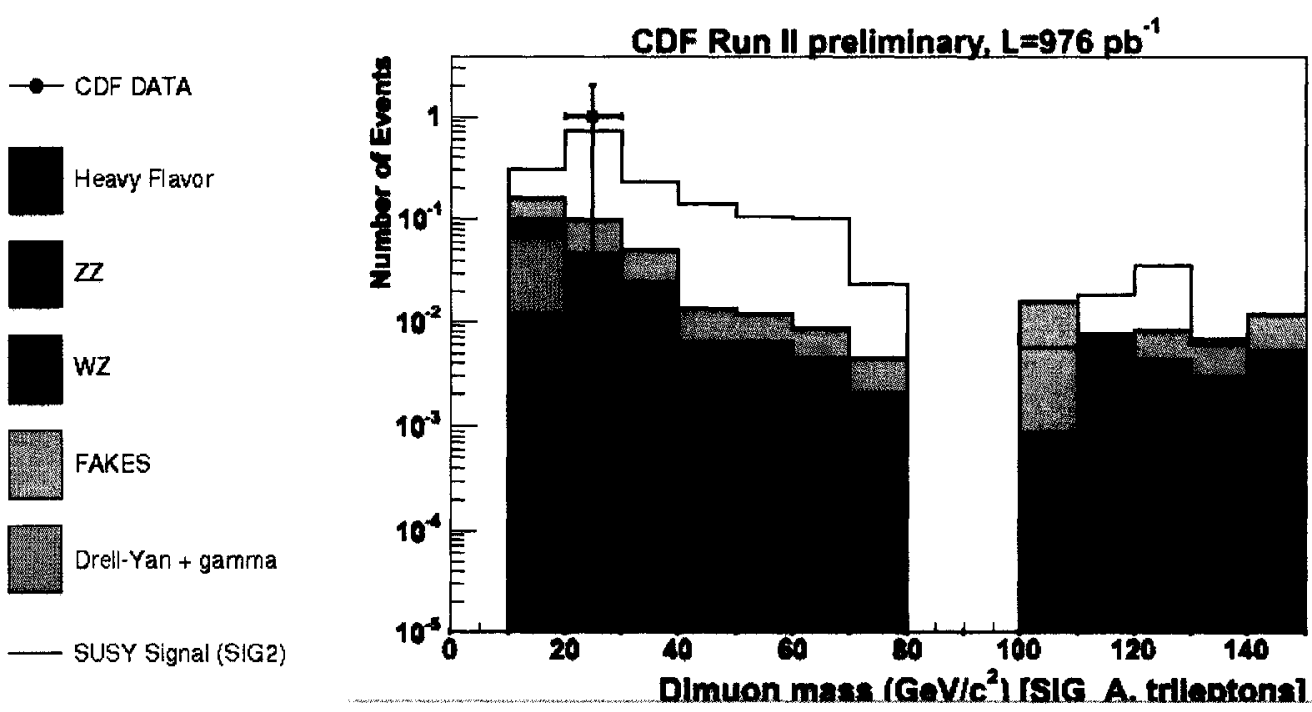

Figure 8.35: Dimuon mass for data, SM prediction, and SUSY MC signal in region SIG_A 
Chapter 8. Trilepton Analysis
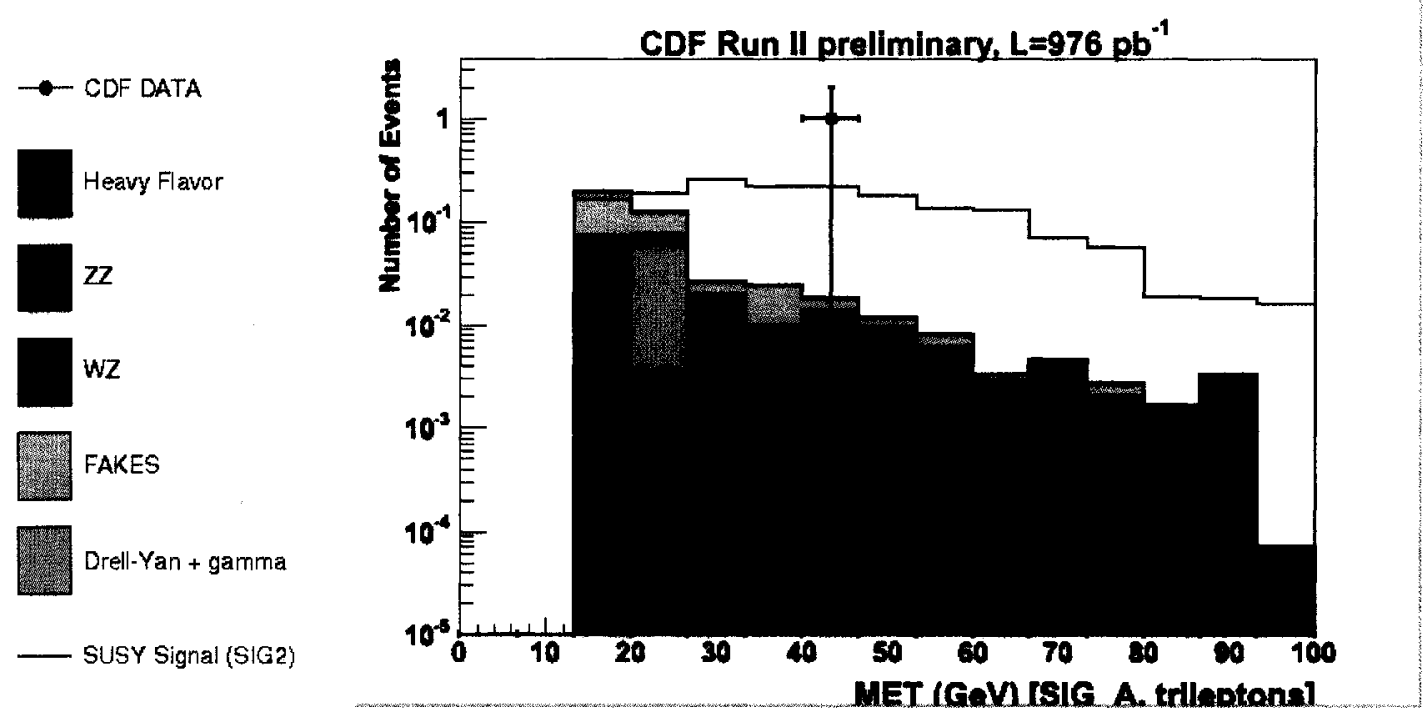

Figure 8.36: $E_{T}$ for data, SM prediction, and SUSY MC signal in region SIG_A 
Chapter 8. Trilepton Analysis

\subsubsection{Control Region SIG_A2}

In this region, we only require $E_{\mathrm{T}}<10 \mathrm{GeV}$. Figures 8.37 to 8.40 show the $M_{\mu \mu}$ and $\mathbb{E}_{\mathrm{T}}$ distributions for data, SM predicted BG, and SUSY signal for dilepton and trilepton case.
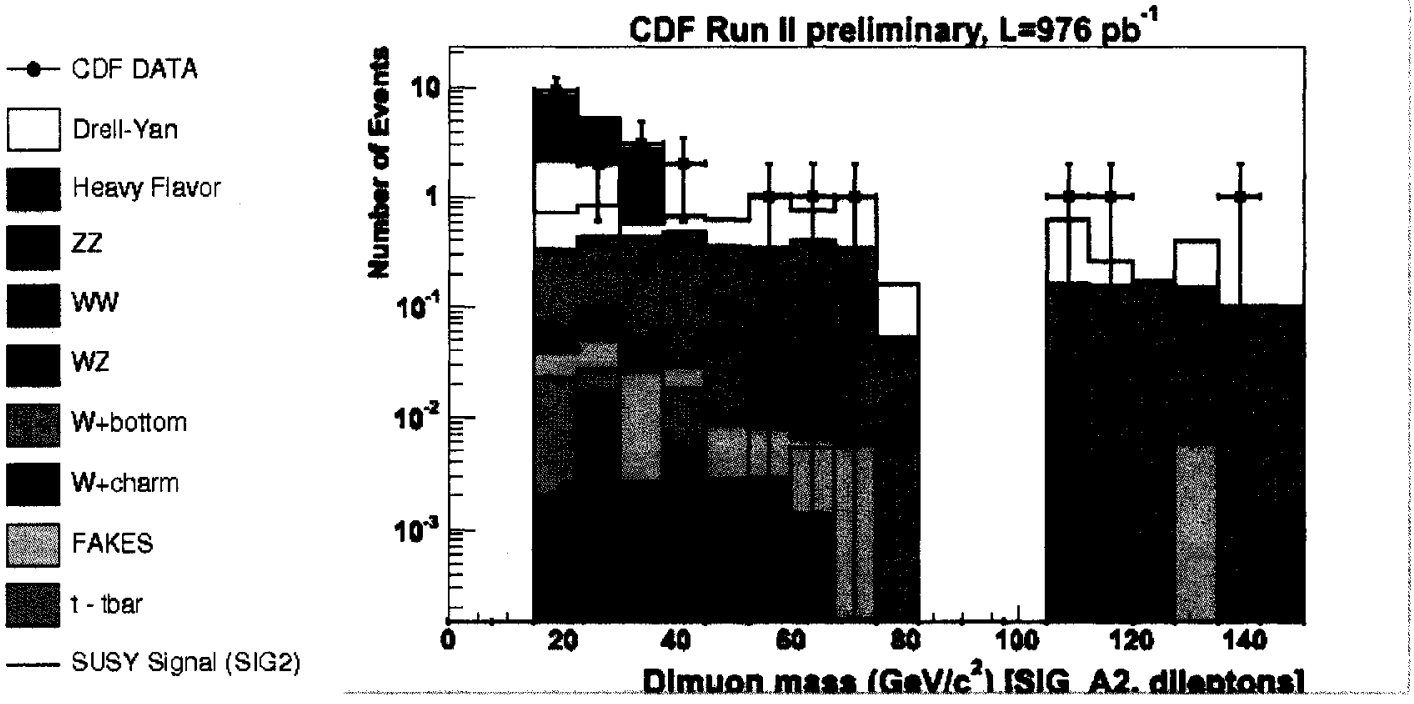

Figure 8.37: Dimuon mass for data, SM prediction, and SUSY MC signal in region SIG_A2 
Chapter 8. Trilepton Analysis
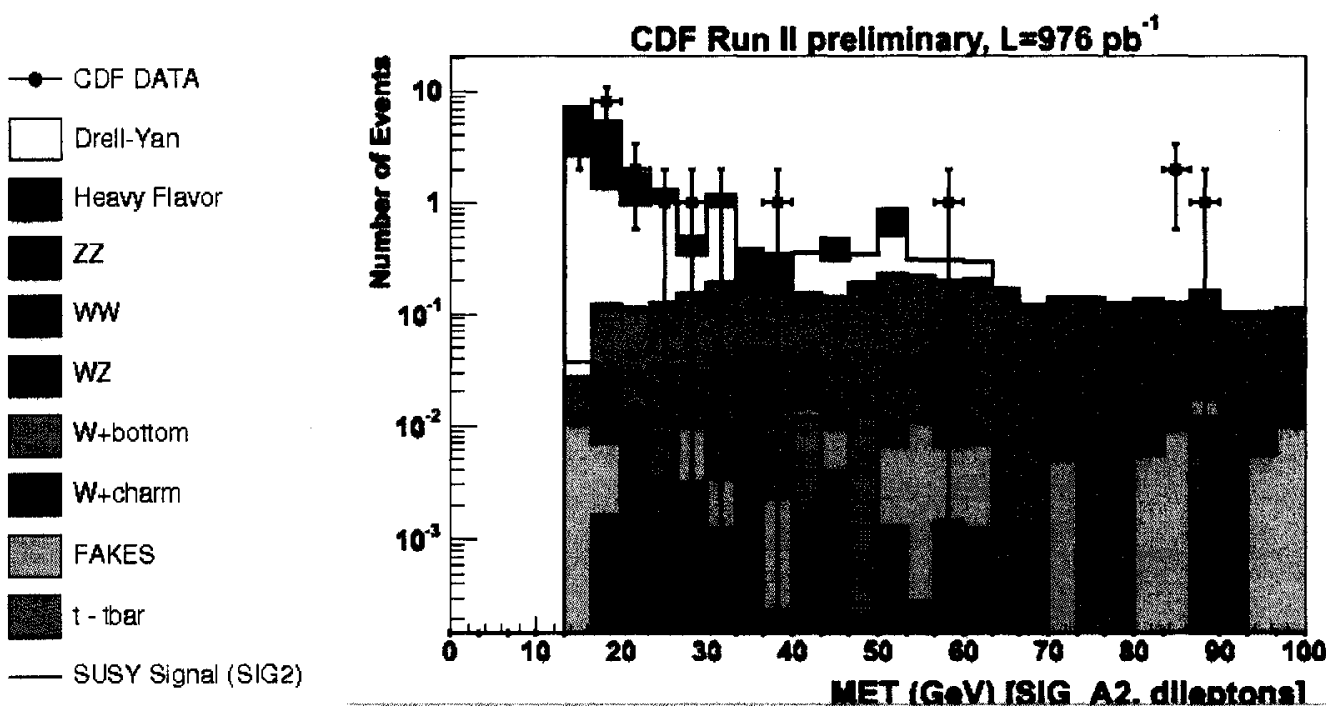

Figure 8.38: $\mathrm{E}_{\mathrm{T}}$ for data, SM prediction, and SUSY MC signal in region SIG_A2
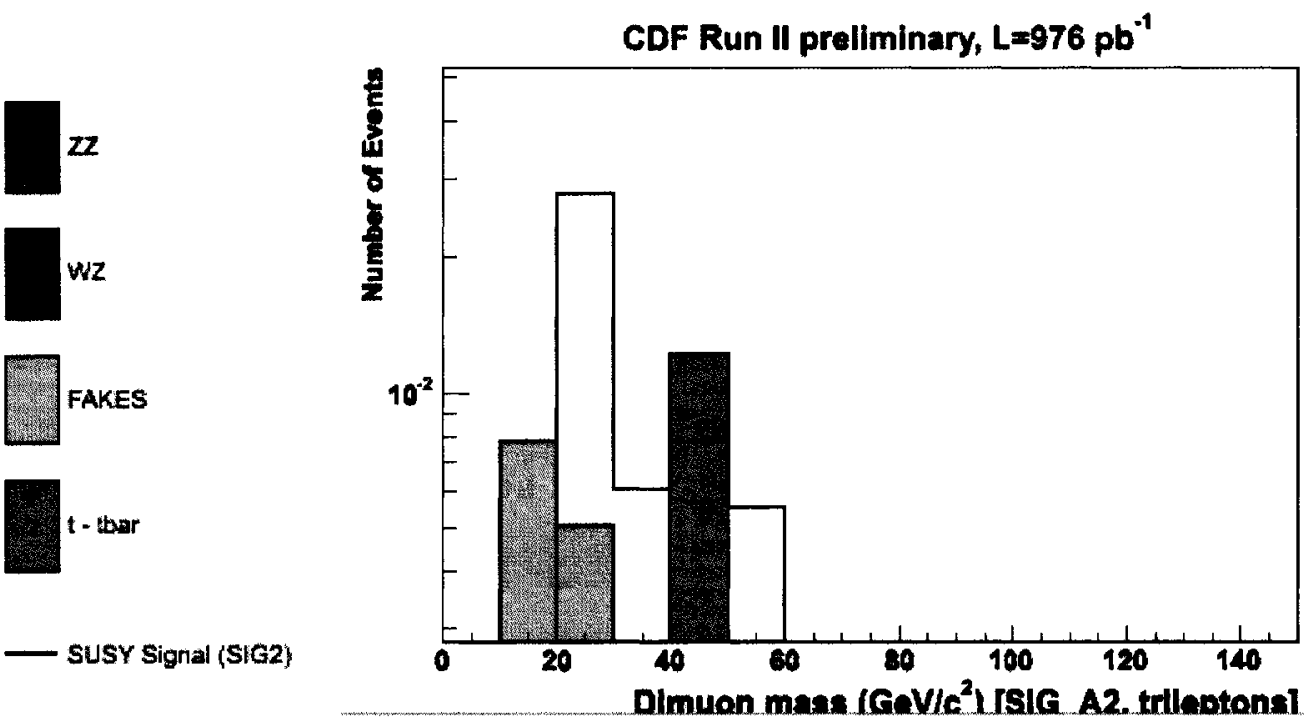

Figure 8.39: Dimuon mass for data, SM prediction, and SUSY MC signal in region SIG_A2 
Chapter 8. Trilepton Analysis

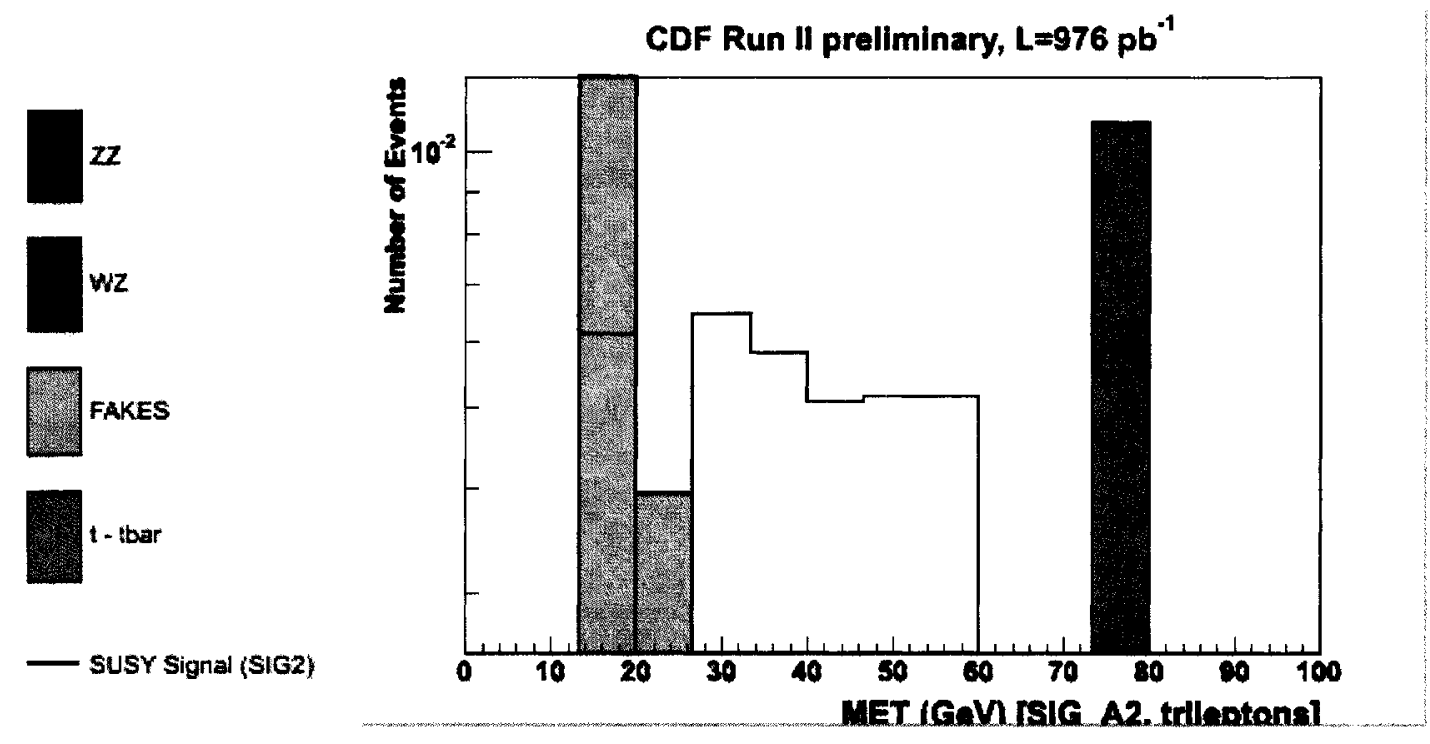

Figure 8.40: $\mathbb{E}_{\mathrm{T}}$ for data, SM prediction, and SUSY MC signal in region SIG_A2 
Chapter 8. Trilepton Analysis

\subsection{Opening the box}

We were confident that we understood the backgrounds after we observed satisfactory agreement between data and SM predictions. We then "opened" the signal "box". That is to say we looked for trilepton events in the signal region SIG_A. We observed one clean event of three isolated muons. In Table 8.11 we list the properties of this event. Figures 8.41 and 8.42 show detector displays of this event. Figures 8.43 to ?? show kinematic variables of SM predictions and our event in SIG_A region where it was observed. Judging from these distributions, our trimuon event does not look like SM background. However, we will treat this event as BG and set up an upper limit in the $\sigma \times B R$, based on this result.

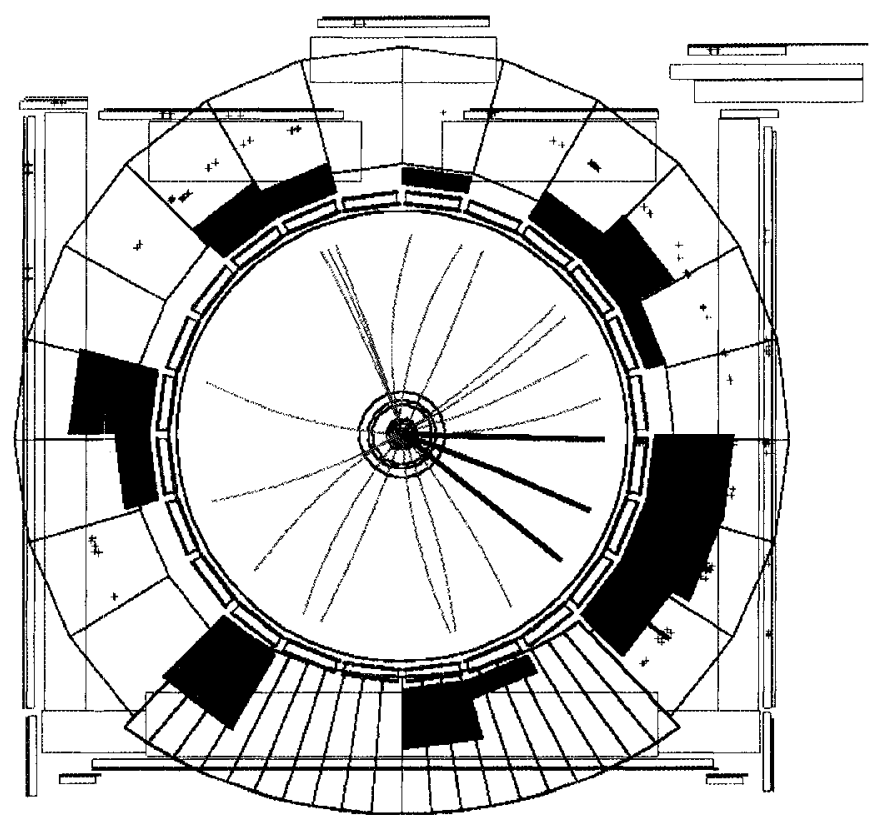

Figure 8.41: Transverse plane display of the observed trimuon event in the CDF detector. Muons are shown in green lines. Only the tracks with $p_{T}>1 \mathrm{GeV} / c^{2}$ are shown (blue lines) and the calorimetry towers with $E_{T}>0.7 \mathrm{GeV}$ 
Chapter 8. Trilepton Analysis

\begin{tabular}{|c|c|}
\hline \multicolumn{2}{|c|}{ Trimuon Event Properties } \\
\hline Run\#, event\# & 211396,9177382 \\
\hline Kind of Leptons & CMUP - CMX - CMX \\
\hline$p_{T}$ of Leptons $(\mathrm{GeV} / \mathrm{c})$ & $45.0,21.1,7.8$ \\
\hline$\eta$ of Leptons & $-0.21,-0.89,0.84$ \\
\hline$\phi$ of Leptons (deg) & $358.8,321.1,340.2$ \\
\hline Isolation of Leptons & 0.05 (frac), 0.009 (frac), 0.13 (frac) and $1 \mathrm{GeV}$ (abs) \\
\hline$Z_{0}(\mathrm{~cm})$ & $7.42,7.23,7.81$ \\
\hline Vertices in event & One, with $Z=7.42 \mathrm{~cm}$ \\
\hline Charge of Leptons & $-1,1,-1$ \\
\hline Number of Lepton silicon hits & $10,10,7$ \\
\hline Dimuon masses $\left(\mathrm{GeV} / c^{2}\right)$ & $28.3(1-2), 21.7(1-3), 25.7(2-3)$ \\
\hline Transverse masses (muon- $\boldsymbol{p}_{T}$ ) & $86.39,51.43,34.15$ \\
\hline 3-d $\Delta \phi($ dimuon) (deg) & 46.3 \\
\hline$\#_{T}(\mathrm{GeV})$ & 43.8 \\
\hline$E_{T} \phi(\mathrm{deg})$ & 205.6 \\
\hline Number of Jet-20 & 1 \\
\hline Jet $E_{T}(\mathrm{GeV})$ & 41.1 \\
\hline Jet $\eta$ & -1.55 \\
\hline Jet $\phi(\mathrm{deg})$ & 102.9 \\
\hline Jet $Z_{0}(\mathrm{~cm})$ & 7.37 \\
\hline
\end{tabular}

Table 8.11: Properties of the observed event in region SIG_A 
Chapter 8. Trilepton Analysis

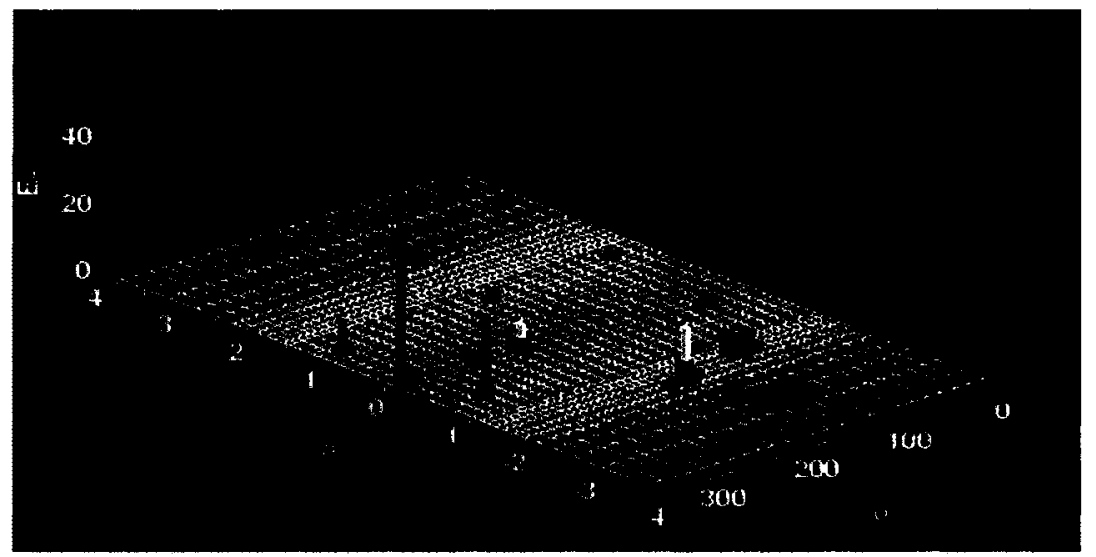

Figure 8.42: $\eta-\phi$ Lego display of $E_{T}$ in the observed trimuon event in the CDF detector. Muons are shown in green lines. Photons are shown in yellow. Only the calorimetry towers with $E_{T}>0.7 \mathrm{GeV}$
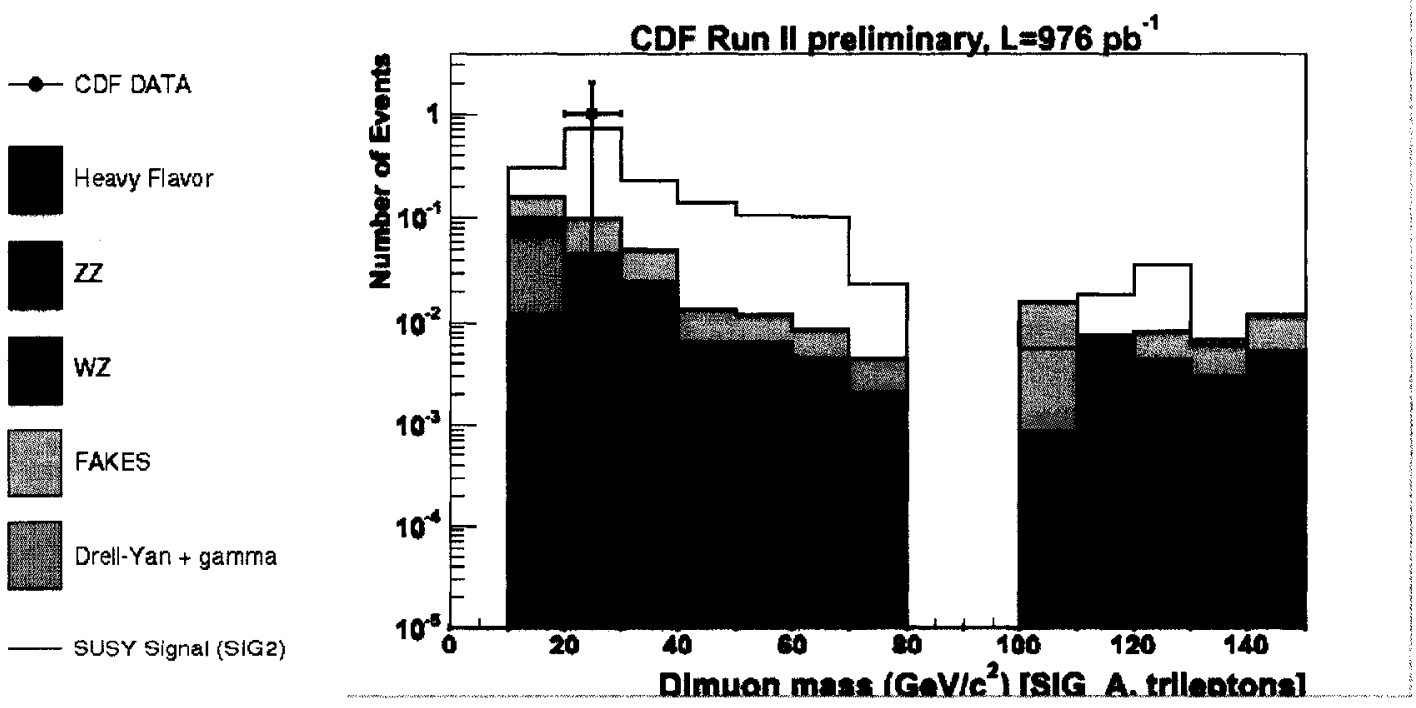

Figure 8.43: Dimuon mass for data, SM prediction, and SUSY MC signal in region SIG_A 
Chapter 8. Trilepton Analysis
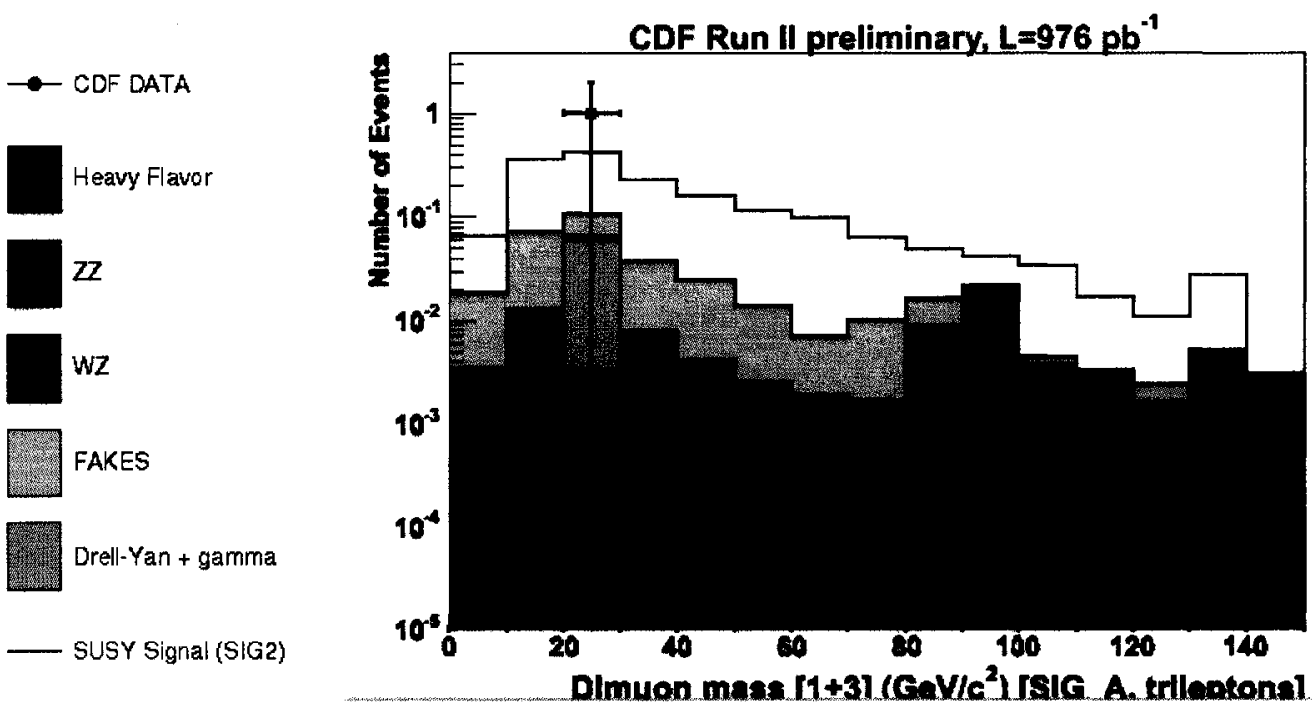

Figure 8.44: Dimuon mass (1+3) for data, SM prediction, and SUSY MC signal in region SIG_A
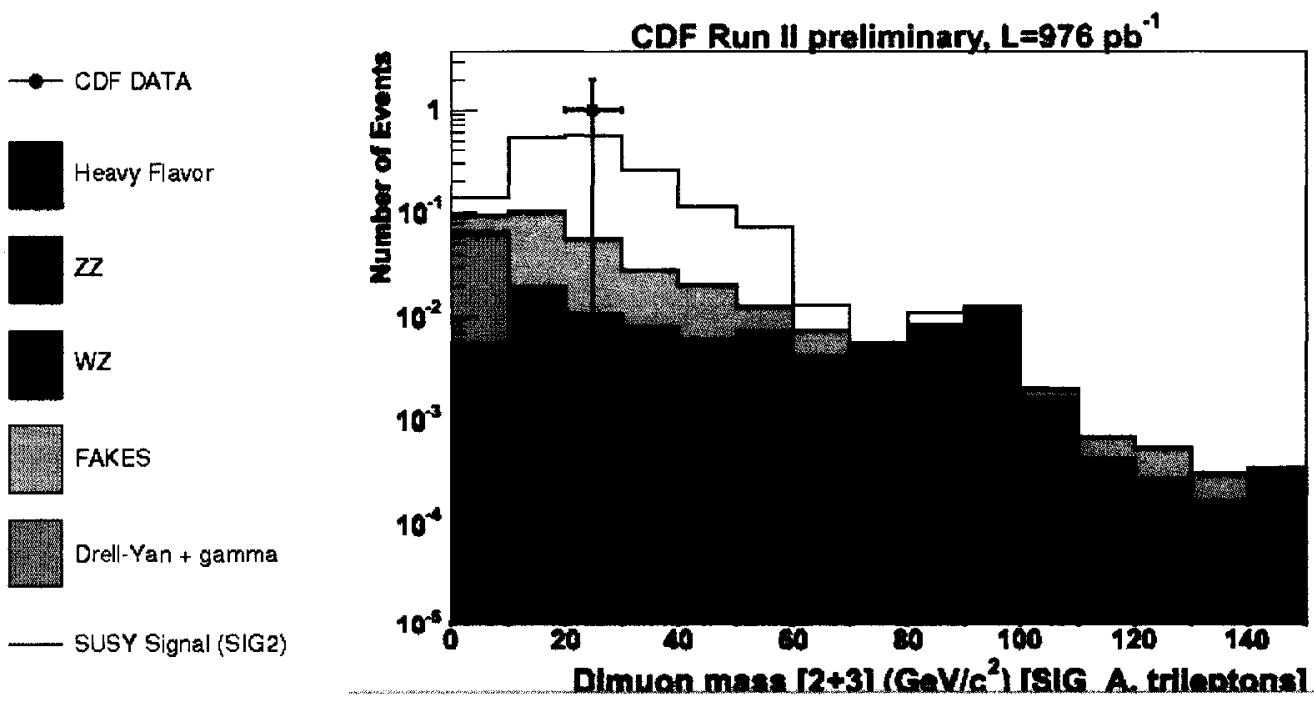

Figure 8.45: Dimuon mass $(2+3)$ for data, SM prediction, and SUSY MC signal in region SIG_A 
Chapter 8. Trilepton Analysis
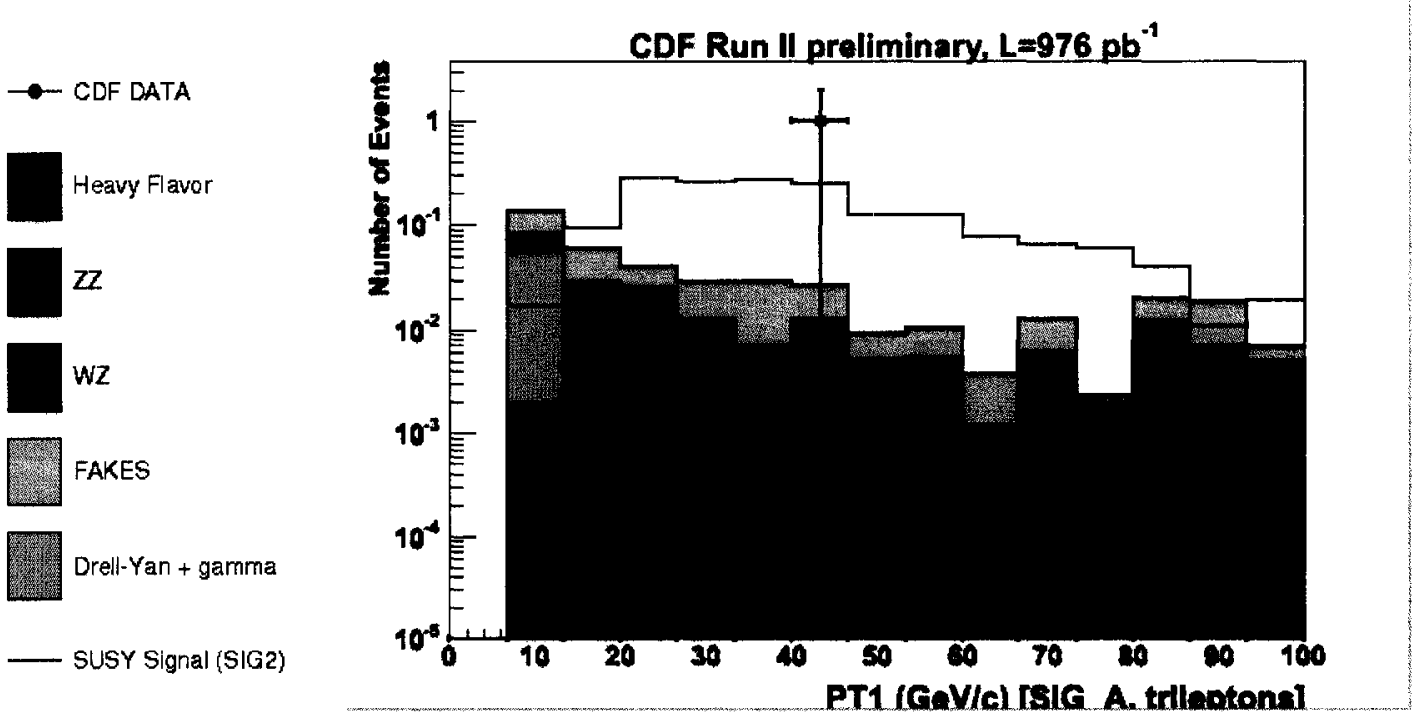

Figure 8.46: $1^{\text {st }}$ muon $p_{T}$ for data, SM prediction, and SUSY MC signal in region SIG_A
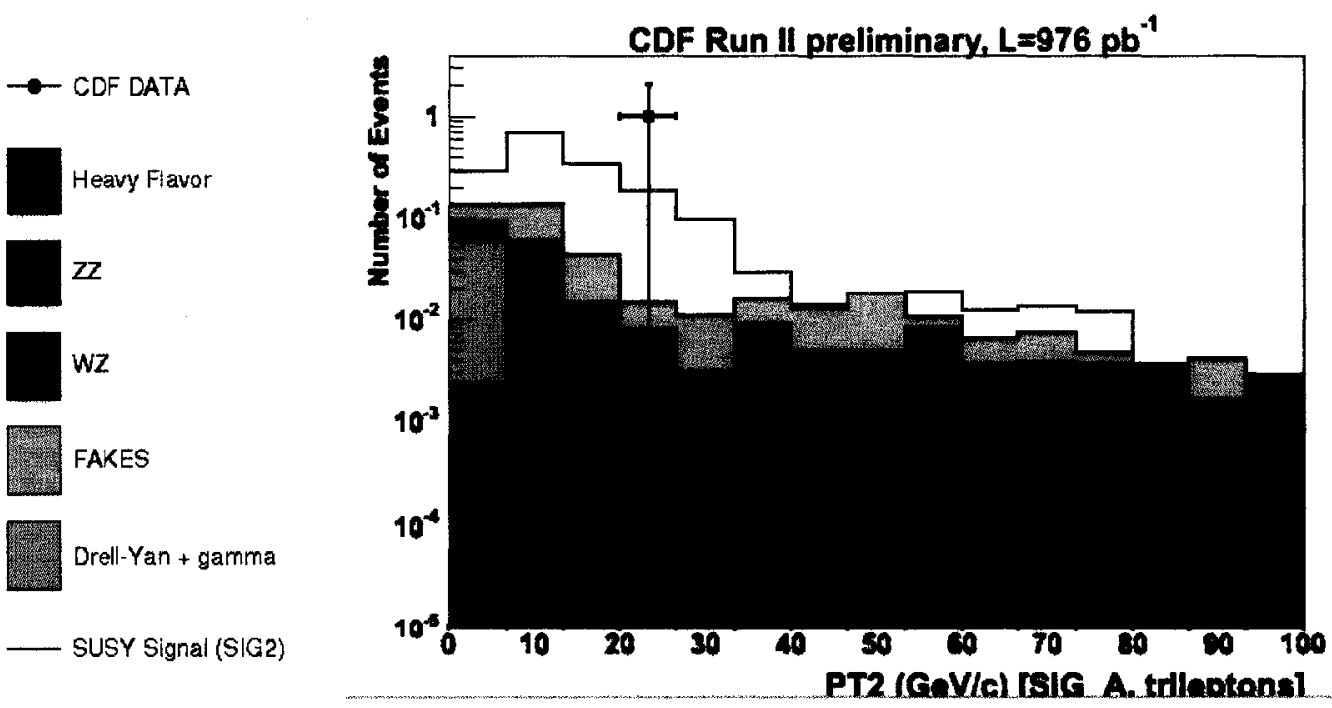

Figure 8.47: $2^{\text {nd }}$ muon $p_{T}$ for data, SM prediction, and SUSY MC signal in region SIG_A 
Chapter 8. Trilepton Analysis
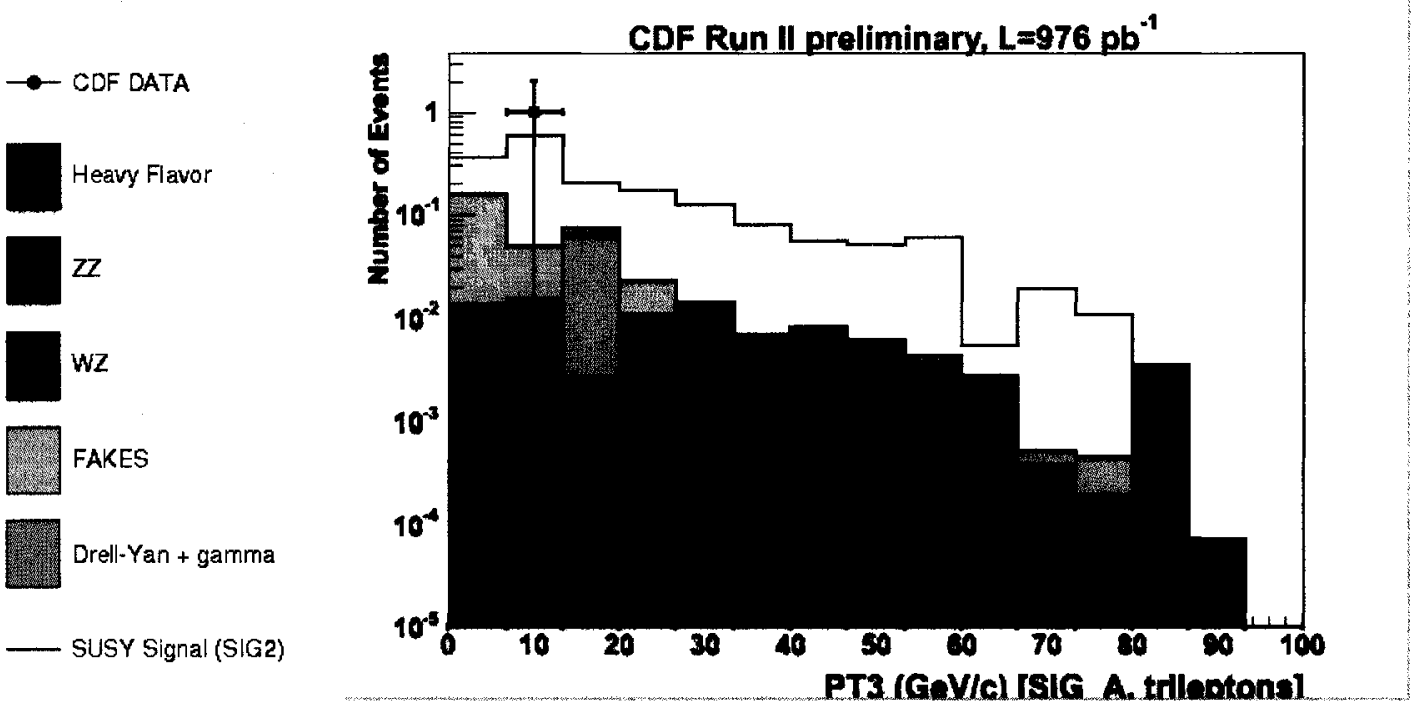

Figure 8.48: $3^{\text {rd }}$ muon $p_{T}$ for data, SM prediction, and SUSY MC signal in region SIG_A
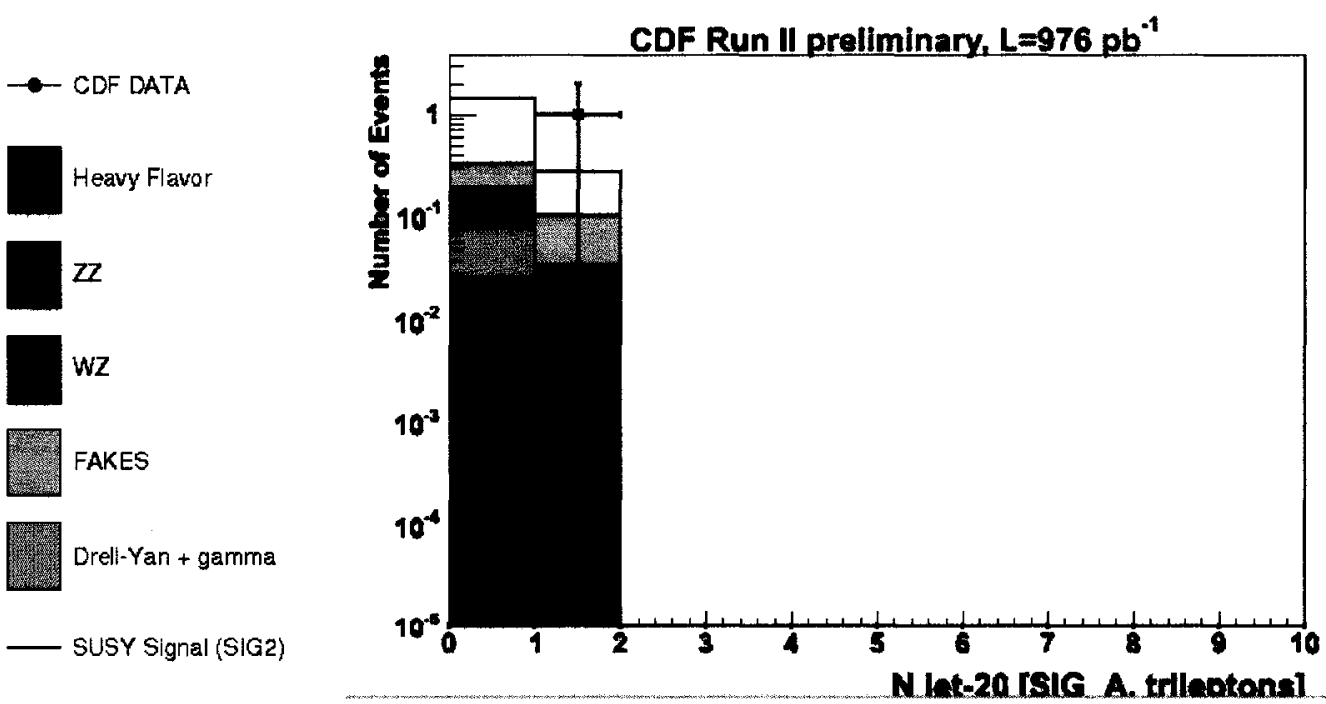

Figure 8.49: Jet multiplicity for data, SM prediction, and SUSY MC signal in region SIG_A 
Chapter 8. Trilepton Analysis
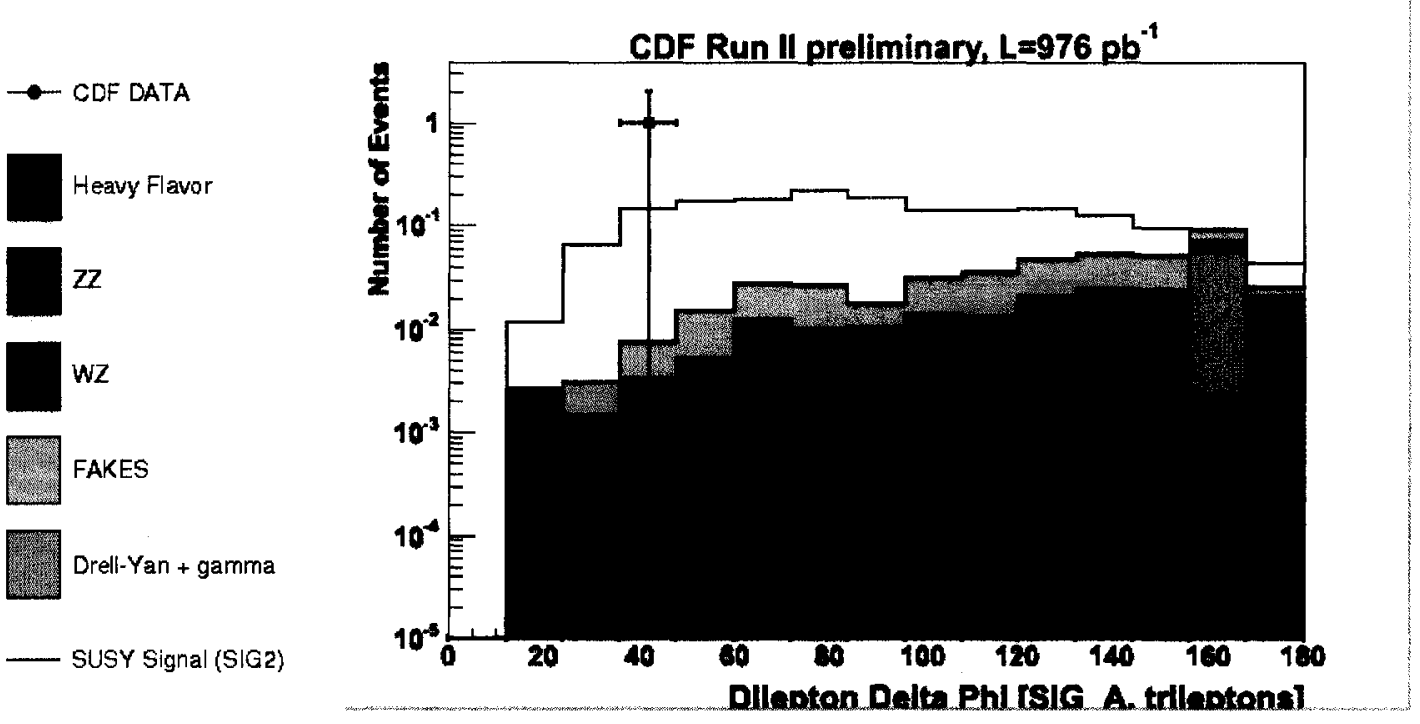

Figure 8.50: $3-\mathrm{D} \Delta \phi$ of the first two muons for data, SM prediction, and SUSY MC signal in region SIG_A 


\section{Chapter 9}

\section{NMH Model}

We conduct a study of the normal scalar mass hierarchy supergravity model (NMH SUGRA). In this theoretical model the generalization universality of the minimal supergravity model (mSUGRA) is relaxed so that the third generation scalar masses are greater than the degenerate first and second generation scallar masses. We perform a scan over the parameter space, report the yields for this model and the acceptances for the Low $p_{T}$ dimuon channel at CDF detector.

\subsection{Introduction}

Recently, in the literature, there have been many reports on serious constraints on mSUGRA models obtained by measurements, such as the anomalous muon magnetic moment $(g-2)_{\mu} / 2$ [15], determination of $b \rightarrow s \gamma$ [?] and the relic CDM density [17]. The (g-2) results call for lighter sleptons while $b \rightarrow s \gamma$ results call for the opposite, heavy squarks. Baer at al [11] reconcile the experimental findings by proposing a SUGRA model with generational mass universality relaxed. Driven by the above constraints 
and the constraints of FCNC which effect only the masses of the first two generatins, they advocate for relatively light (few hundred $\mathrm{GeV}$ ) degenerate the first and second generation scalar masses and much heavier (multi-TeV) third generation scalar mass. Constraints from $B_{d}-\bar{B}_{d}$ allow for low values of $m_{1 / 2}$. With all the above constraints taken into account significant portion of the parameter space still remains available.

In this NMH model, due to light slepton masses in the first two generations, chargino and neutralino decays into electron and muons are enhanced, leading to large rates for isolated multileptons plus $\mathbb{E}_{\mathrm{T}}$ plus jets, a golden signature at collider detectors such as CDF.

Our plan is to set a limit on this model with the results obtained in trilepton search at SUSY with $1 \mathrm{fb}^{-1}$. 
Chapter 9. NMH Model

\subsection{Generation}

To generate NHM SUGRA events, we use the executable isasugra from isajet package, version 7.74. In particular, we generate mass spectra and decay modes of NHM SUGRA particles, stored in a file in a special format of SUSY Les Houche Accord (SLHA). The mass spectra and decay modes are then used as input to Pythia which does the rest of the generation.

We have created mass spectra and particle decay tables in the SLHA file format for many points in the parameter space varying: $\mathrm{m}_{0}(50-200)$ in steps of $10, \mathrm{~m}_{1 / 2}(100$ $300)$ in steps of 10 , and $\tan (\beta)(3-30)$ in steps of 3 . We keep $\mathrm{A}_{0}=0, \mu=1$, and top mass $\mathrm{m}_{t}=175 \mathrm{GeV}$. Setting of $\mathrm{m}_{0}$ sets the common scalar masses, but we then set the third generation scalar mass $m_{0}(3)=1400 \mathrm{GeV}$, as suggested by authors in [11].

For the sake of illustration, bellow is an excerpt from the menu given when executing isasugra.

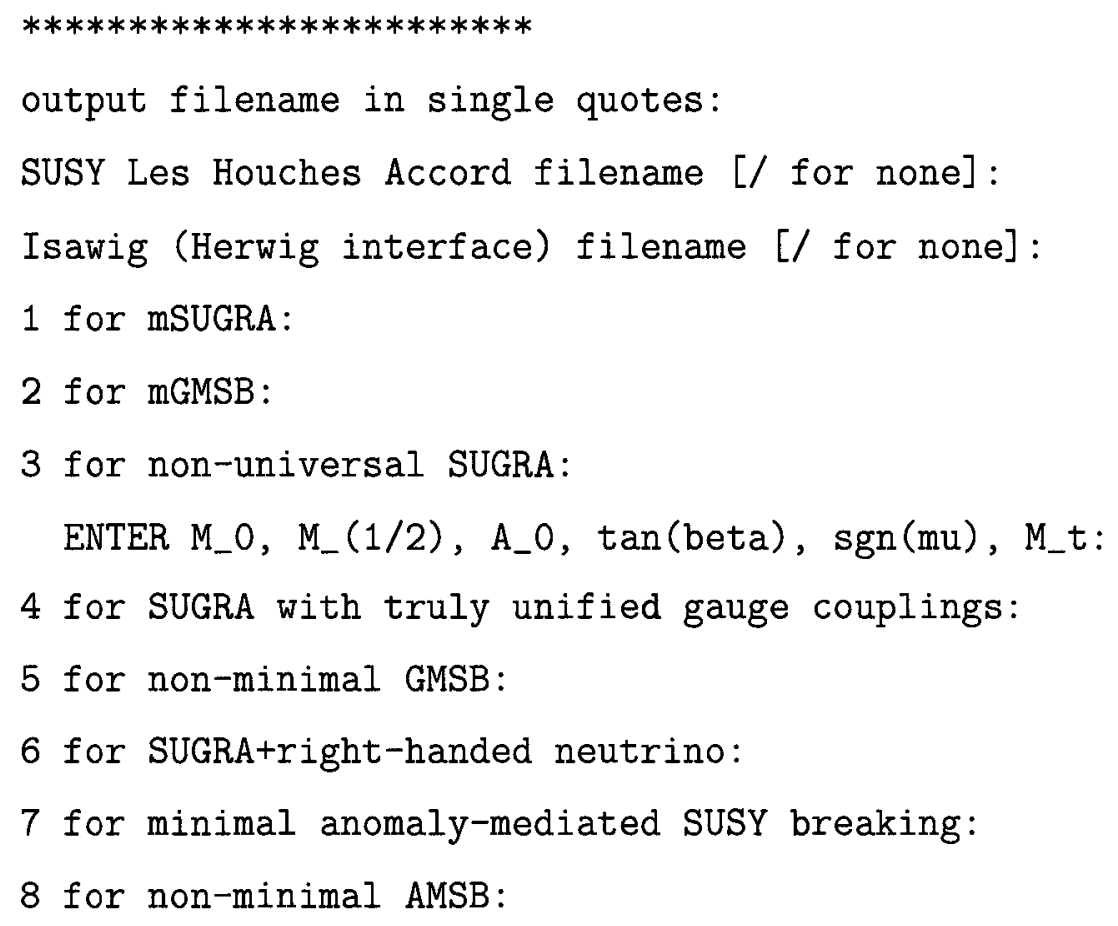


Chapter 9. NMH Model

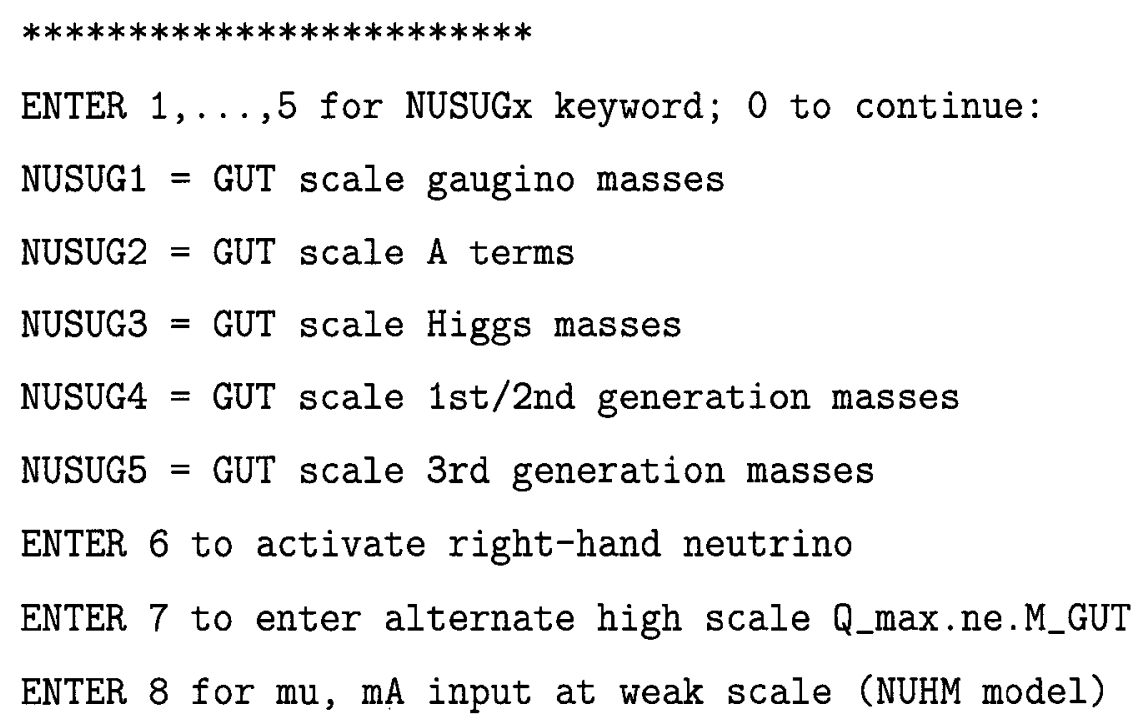

We feed the mass spectrum of the particles at the GUT scale and the particle decay table into Pythia, which is a part of cdf Sim executable, which in turn is part of mcProduction package of cdf soft. Motivated by a scan of the NMH parameter phase spaces shown in Figure 11, on page 20 of [11], we have generated (with Pythia) and ran mcProduction on some of the first fourteen points which have the highest charginoneutralino production cross section. In NMH model these points are described by parameters $\mathrm{A}_{0}=0, \mu>0, \mathrm{~m}_{t}=175 \mathrm{GeV}, \mathrm{m}_{0}(3)=1400 \mathrm{GeV}, \mathrm{m}_{1 / 2}=225$, and $\mathrm{m}_{0}=$ $[50,140]$ in steps of 10 . The authors of [11] use $\tan (\beta)=10$, and we use $\tan (\beta)=$ 9. For each of these points in the parameter phase space we have generated and produced about $50 \mathrm{~K}$ events. In the table 9.1 we list the exact number of events we have available for each of the points, the location of the output of MCProduction jobs and the corresponding log files. The files that contain mass spectra and decay modes of generated particles used as the input to Pythia are labeled NMH.slha and are located in the directories listed in this table. Further, we have ntupled the produced data

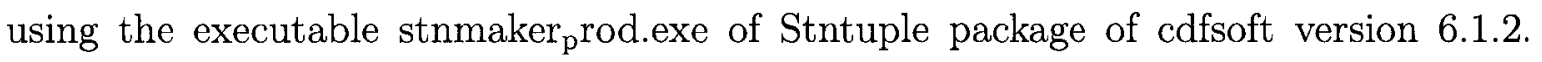
The location of these ntuples is listend in the table 9.3 . 
Chapter 9. NMH Model

\begin{tabular}{|c|c|c|c|c|}
\hline $\mathrm{m}_{0}$ & events & output directory & files & log files \\
\hline 50 & 49120 & m0_50/rexo0b.000*.output/ & rb024f8b.* & exo0b.000*.MCProd.*.log \\
\hline 60 & 49120 & m0_60/rexo0b.000*.output/ & rb024f8b.* & exo0b.000*.MCProd.*.log \\
\hline 70 & 41963 & m0_70/rexo0b.000*.output/ & rb024f8b.* & exo0b.000*.MCProd.*.log \\
\hline 80 & 49620 & m0_80/rexo0b.000*.output/ & rb024f8b.* & exo0b.000*.MCProd.*.log \\
\hline 90 & 0 & m0_90/rexo0b.000*.output/ & NA & NA \\
\hline 100 & 49120 & m0_100/rexo0b.000*.output/ & rb024f8b.* & rexo0b.000*.MCProd.*.log \\
\hline 110 & 48000 & m0_110/rexo0b.000*.output/ & rb024f8b.* & rexo0b.000*.MCProd.*.log \\
\hline 120 & 0 & m0_120/rexo0b.000*.output/ & NA & NA \\
\hline 130 & 49120 & m0_130/rexo0b.000*.output/ & rb024f8b.* & rexo0b.000*.MCProd.*.log \\
\hline 140 & 23620 & m0_140/rexo0b.000*.output/ & rb024f8b.* & rexo0b.000*.MCProd.*.log \\
\hline 150 & 0 & m0_150/rexo0b.000*.output/ & NA & NA \\
\hline
\end{tabular}

Table 9.1: For each $m_{0}$ point, number of events generated and produced using cdfsoft MCProduction, and a location of corresponding subdirectory, sequential output files and $\log$ files in this subdirectory of directory fcdfdata121:/export/data3/exotic/rekovic/rexo0b/. For points $\mathrm{m}_{0}=90,120,150 \mathrm{CAF}$ jobs crashed, so the output sequential files are not available.

\begin{tabular}{|rl|}
\hline $\mathrm{m}_{0}$ & stntuple files \\
\hline \hline 50 & stn_NUSM_m0_50_m12_225_tanb_9_mu_1_m03_1400_slha.root* \\
\hline 60 & stn_NUSM_m0_60_m12_225_tanb_9_mu_1_m03_1400_slha.root \\
\hline 70 & stn_NUSM_m0_70_m12_225_tanb_9_mu_1_m03_1400_slha.root \\
\hline 80 & stn_NUSM_m0_80_m12_225_tanb_9_mu_1_m03_1400_slha.root* \\
\hline 100 & stn_NUSM_m0_100_m12_225_tanb_9_mu_1_m03_1400_slha.root* \\
\hline 110 & stn_NUSM_m0_110_m12_225_tanb_9_mu_1_m03_1400_slha.root* \\
\hline 130 & stn_NUSM_m0_130_m12_225_tanb_9_mu_1_m03_1400_slha.root* \\
\hline 140 & stn_NUSM_m0_140_m12_225_tanb_9_mu_1_m03_1400_slha.root* \\
\hline
\end{tabular}

Table 9.2: For each $\mathrm{m}_{0}$ point, location of root files generated using cdfsoft Stntuple_v_243, in the directory fcdflnx6:/ export/ data3/ cdfmc/ rekovic/ MC/ $6.1 .2 / \mathrm{rexo0b} /$. 
Chapter 9. NMH Model

\begin{tabular}{|rcccc|}
\hline $\mathrm{m}_{0}$ & $\sigma_{\text {THtotal }}[\mathrm{fb}]$ & $L_{M C}\left[\mathrm{fb}^{-1}\right]$ & $3 \mu$ anlysis & expected (with $1 \mathrm{fb}^{-1}$ ) \\
\hline \hline 50 & 77.8 & 631.4 & 0 & 0 \\
\hline 60 & 79.1 & 621.0 & 0 & 0 \\
\hline 70 & 79.0 & 531.2 & 359 & 0.68 \\
\hline 80 & 79.8 & 621.8 & 529 & 0.85 \\
\hline 100 & 79.8 & 615.5 & 6 & 0.01 \\
\hline 110 & 76.0 & 631.6 & 9 & 0.015 \\
\hline 130 & 81.7 & 601.2 & 10 & 0.016 \\
\hline 140 & 80.5 & 293.4 & 7 & 0.023 \\
\hline
\end{tabular}

Table 9.3: For each $\mathrm{m}_{0}$ point of the NMH model with above described parameters, theoretical cross section given by PYTHIA, luminosity of MC sample (i.e. number of events generated $/ \sigma_{T H}$ ), number of tri-muon events that pass cuts of our analysis in low $p_{T}$ di-muon channel, and number of expected events to be observed with $1 \mathrm{fb}^{-1}$ data collected with CDF at Tevatron. 


\section{Chapter 10}

\section{References}




\section{References}

[1] Steven Weinberg, The Quantum Theory of Fields, Volume II, Cambridge (1995).

[2] Steven Weinberg, The Quantum Theory of Fields, Volume III, Cambridge (1995).

[3] LEPColl LEP Collaboration hep-ex/0512014, LEP Electroweak Workign Group web page http://www.cern.ch/LEPEWWG

[4] UTfitColl UTfit Collaboration, M. Bona et al. J. High Energy Phys 0507, 028 (2005)

[5] I. Buchbinder and S. Kuzenko, Ideas and Methods of Supersymmetry and Supergravity, IOP Publishing (1998).

[6] J. Wess and B. Zumino, A lagrangian model invariant under supergauge transformations, Phys. Lett. B78 1 (1974).

[7] G. Jungman and M. Kamionkowski, Gamma rays from neutralino annihilation, Phys. Rev. D51, 3121 (1995).

[8] L. Susskind, Supersymmetry, Phys. Rept. 104, 181 (1984).

[9] S. Martin, A Supersymmetry Primer, hep-ph/9709356

[10] L. Girardello and M.T. Grisaru, Nucl. Phys, B194, 65 (1982)

[11] H. Baer, A. Belyaev, T. Krupovnickas and A. Mustafayev, Normal Scalar Mass Hierarchy Reconciles $(g-2)_{\mu}, b \rightarrow s \gamma$ and Relic Density hep-ph/0403214 (2004)

[12] H. Baer, A. Mustafayev, S. Profumo, A. Belyaev, X. Tata, Direct, Indirect and Collider Detection of Neutralino Dark Matter In SUSY Models with Non-universal Higgs Masses hep-ph/0504001 (2005) 
References

[13] V. Berezinsky, A. Bottino, J. Ellis, N. Fornengo, G. Mignola, S. Scopel Neutralino Dark Matter in Supersymmetric Models with Non-Universal Scalar Mass Terms "hep-ph/9508249 (2002)"

[14] J Ellis, KA Olive, Y Santoso, The MSSM Parameter Space with Non-Universal Higgs Mass, hep-ph/0204192 (2002)

[15] G. Bennett et al. (E821 Collaboration), Measurement of the Negative Muon Anomalous Magnetic Moment to 0.7 ppm, Phys. Rev. Lett, 89 (2002) 101804 and hep-ex/0401008

[16] M. Davier, S. Eidelman, A. Hocker and Z. Zhang, "Eur. Phys. J" C 31 (2003) 503

[17] D.N. Spergel etal, Determination of Cosmological Parameters, "Astrophys. J." 148 (2003) 175

[18] V. Barger, C.E.M. Wagner, etal, Report of the SUGRA Working Group for Run II of the Tevatron, hep-ph/0003154.

[19] D. Bortoletto etal, Trigger Efficiency for medium $p_{T}$ Dimuon Triggers in Run II, CDF Note 7196 8/15/04

[20] S. Krutelyov et al, Measurement of Level-1,2 and 3 Low- $p_{T}$ Dimuon Trigger Efficiencies for the $B_{s}^{0} \rightarrow \mu^{+} \mu^{-}$Analysis, CDF Note $73141 / 25 / 05$.

[21] Official CDF Good Run web page, http://wwwcdf.fnal.gov/internal/dqm/goodrun/good.html.

[22] D.Bortoletto, A.Canepa, O.Gonzalez, E.Lytken, Trigger Efficiency for medium $p T$ Dimuon Triggers in Run II, CDF Note 7196, 8/15/04

[23] Mario Paolo Giordani and Stephan Lammel, Dilepton Triggers for Exotic Searches at CDF II , CDF Note 6074, 8/8/02.

[24] M. Gold and V. Rekovic Muon ID efficiencies and Scale Factors for Exotics Searches CDF Note 7210, 11/23/04.

[25] M. Gold, V. Rekovic and J. Strologas, Low p p Muon ID Efficiencies in Data Gen6 for Exotics Searches CDF Note 8307, 05/10/06.

[26] Daniel Whiteson, Muon Momentum Resolution in 5.3.1 Data and Simulation, CDF Note 7254, 2004.

[27] Giulia Manca, Martin Griffiths, Beate Heinemann, Searches for Chargino and Neutralino in the e+e/mu+e/mu with 1 fb-1 of data, CDF Note 8389, 7/19/06. 
References

[28] Martin Griffiths, Beate Heinemann, Giulia Manca, Fake Rate For Low- $p_{T}$ Leptons, CDF Note 7470, Date: 2/5/05.

[29] The package stnEvent contains the cut classes muId and eleId. When added to the offline (with appropriate softlinks), the "make stnEvent.nobin" command produces a ROOT-loadable library. The stnEvent package is available in the cvs repository gold/stnEvent, obtained with the command "cvs checkout gold/stnEvent".

[30] Eric James, private communication. The code is implemented for Stntuple formatted data by the class stnTrig in the stnEvent package.

[31] Vyachelsav Krutelyov, private communication.

[32] http://www-cdf.fnal.gov/internal/fastnavigator/fastnavigator.html.

[33] M. Gold, V. Rekovic Muon ID efficiencies and Scale Factors for Exotics Searches, CDF Note 7210, 11/23/04.

[34] $J / \psi$ and $\psi(2 S)$ Production in $p-\bar{p}$ Collisions at $\sqrt{s}=1.8$ TeVCDF Collaboration, Physical Review Letters, vol 79, page 572, 1997.

[35] Upsilon Production and Polarization in $p-\bar{p}$ Collisions at $\sqrt{s}=1.8$ TeV CDF Collaboration, Physical Review Letters, vol 88, 2002.

[36] M. Gold, V. Rekovic, J. Strologas, Measurement of Level 1, 2 and 3 Low $p_{T}$ Dimuon Trigger Efficiency for the SUSY Trilepton Search in $\mu \mu$ Channel for Gen6 Data at CDF CDF Note, 05/10/06.

[37] U. Grundler, A. Taffard, X. Zhang, High pt muons, recommended cuts and efficiencies for Winter 2006, CDF Note 7956 12/15/2005. 


\section{Appendices}




\section{Appendix A}

\section{Acceptance with $z \operatorname{Min} A x S L$}

In the figure below, we show the loss of acceptance, which is about $1 / 6$, if zMinAxSL cut is imposed. This cut improves the trigger efficiency by about 4 percent, but the loss of acceptance is too great. 
Appendix A. Acceptance with $z \operatorname{Min} A x S L$
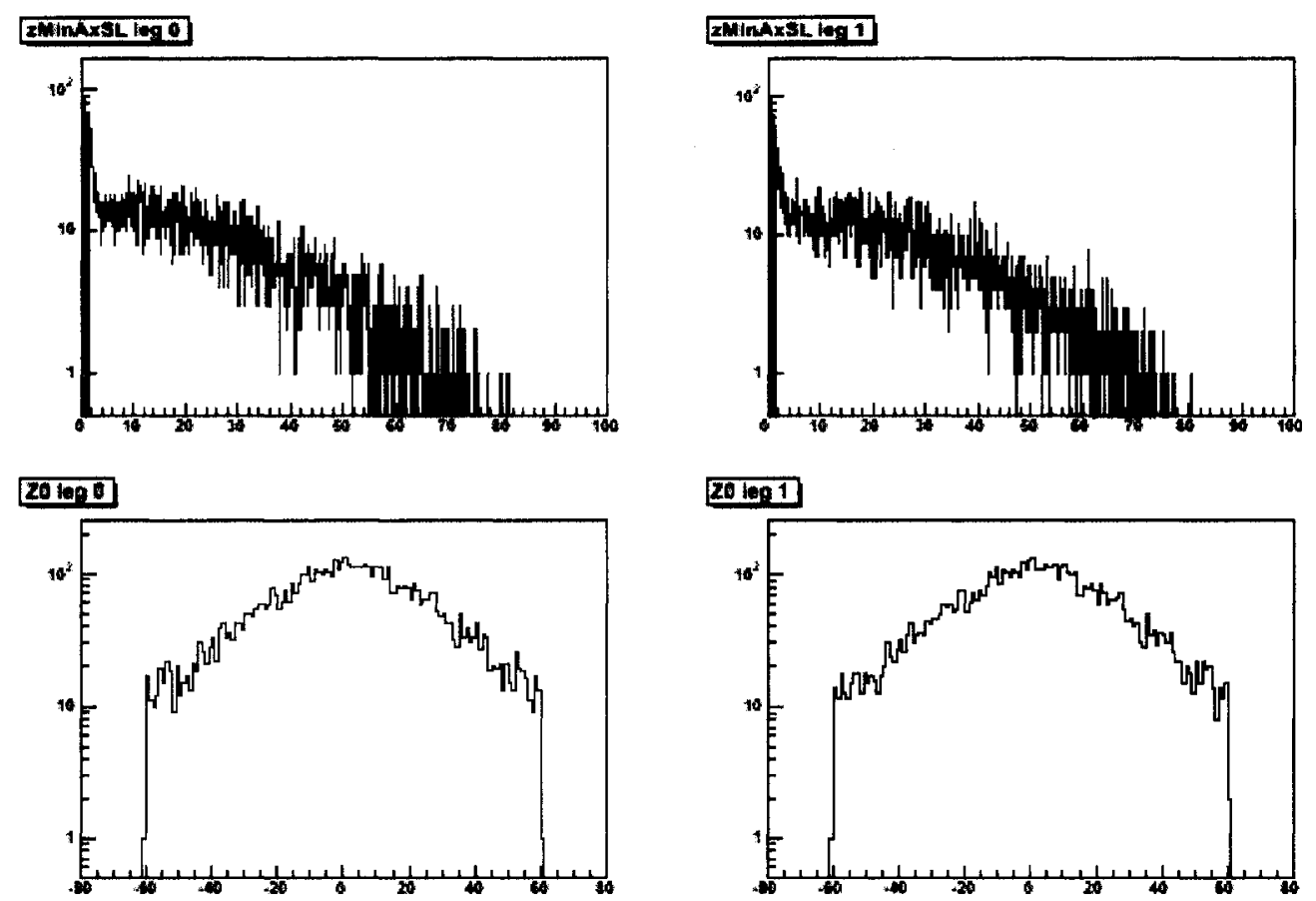

Figure A.1: Top: zMinAxSL, in green events with zMinAxSL $<1.5 \mathrm{~cm}(1 / 6$ of total), for TRIGGER and OTHER leg. Bottom: z0 for TRIGGER and OTHER leg 


\section{Appendix B}

\section{Muon Isolation Efficiency as a}

\section{Function of Openning Angle in}

\section{gen5 Data}

In this appendix we provide a plot in Figure B.1 we show and Isolation efficiency vs opening angle segment $\Delta \phi\left(1, \mathrm{j}_{2}\right)$. In this study, we used the data and MC from all six $\Delta \phi\left(\mathrm{l}, \mathrm{j}_{2}\right)$ segments $\left(150^{\circ}-180^{\circ}\right)$ so to gain in statistics for HF LS background. 
Appendix B. Muon Isolation Efficiency as a Function of Openning Angle in gen5 Data

Isolation efficiencie vs Dphi opening angle

Mon Dec 20 18:12:35 2004

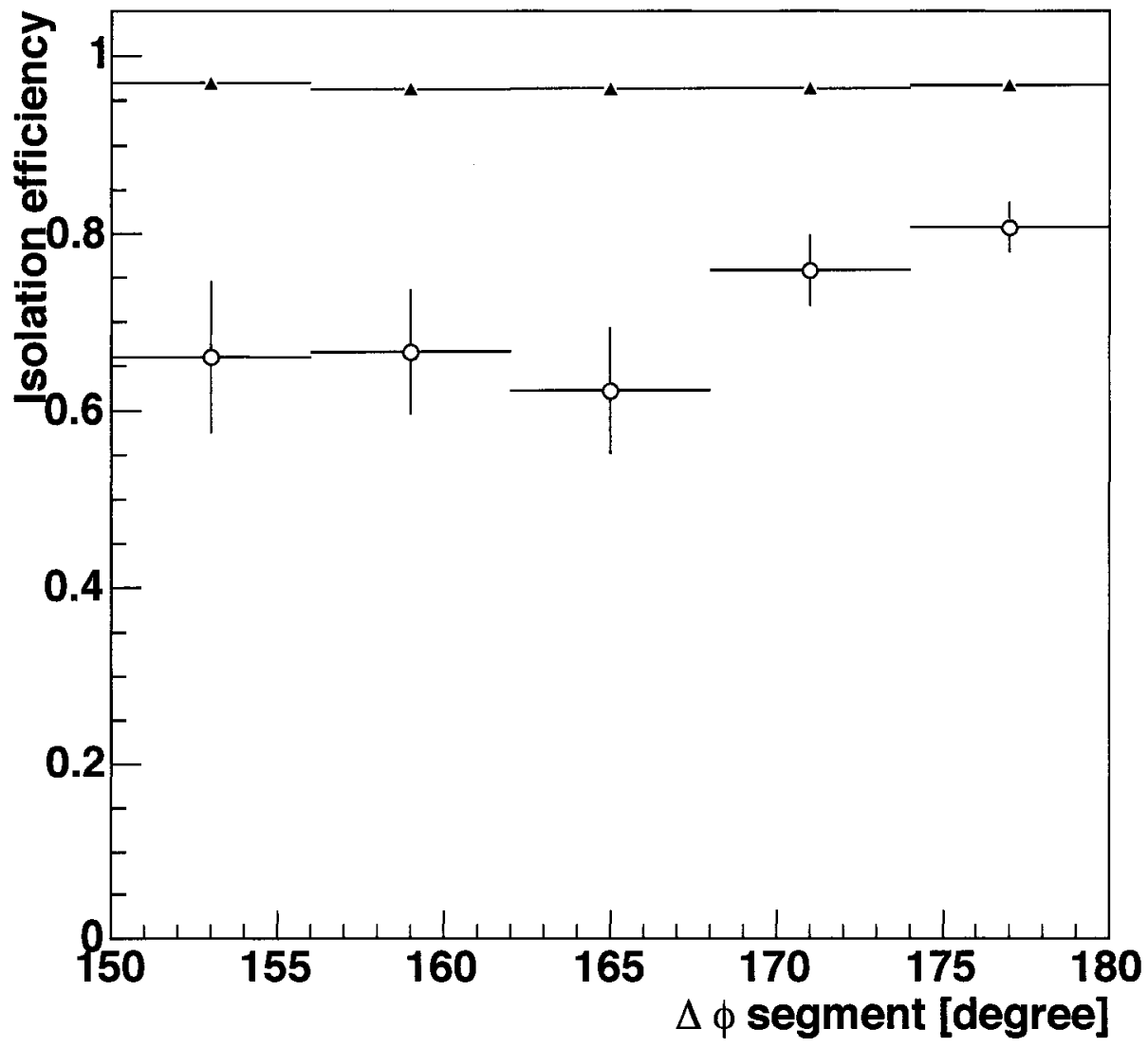

Figure B.1: Isolation efficiency vs $\Delta \phi\left(1, \mathrm{j}_{2}\right)$ segment. Fixed energy cut of 2 for $\mathrm{p}_{T}<20$ $\mathrm{GeV} / \mathrm{c}$, otherwise ConeR4Et $/ p_{T}<0.1$ cut. 


\section{Appendix C}

\section{Resonances Used for gen6 Data ID Efficiencies}

In the figures in this appendix, we show the mass resonances of $J / \psi, \Upsilon$ and $\mathrm{Z}$ in $p_{T}, \phi$ and $\eta$ bins, used in the calculations of efficiencies. 
Appendix C. Resonances Used for gen6 Data ID Efficiencies
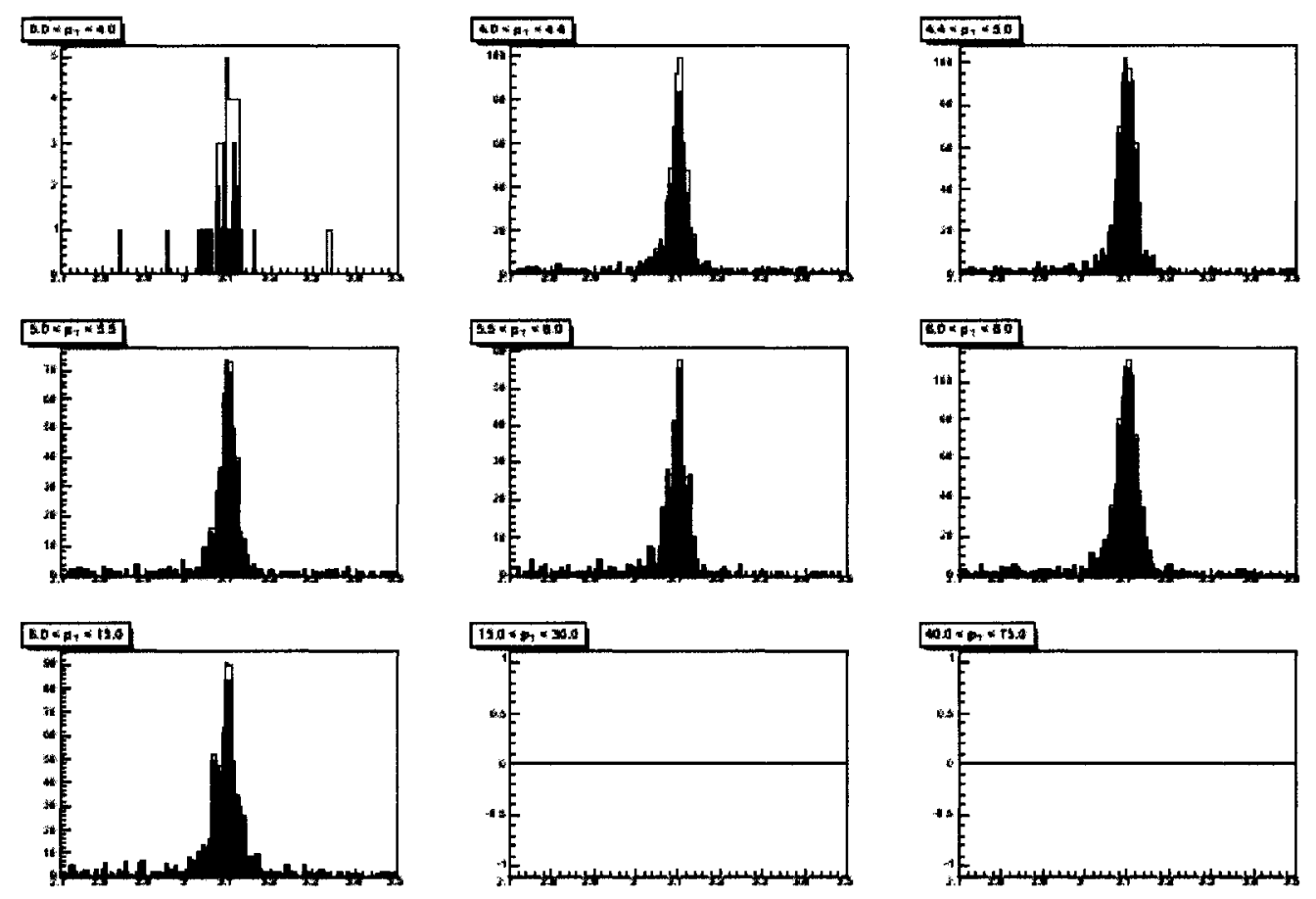

Figure C.1: $J / \psi$ mass distributions in pt bins, used in efficiency calculations. Numerators are in blue, filled, histograms, and denominators in red, not filled, histograms. 
Appendix C. Resonances Used for gen6 Data ID Efficiencies
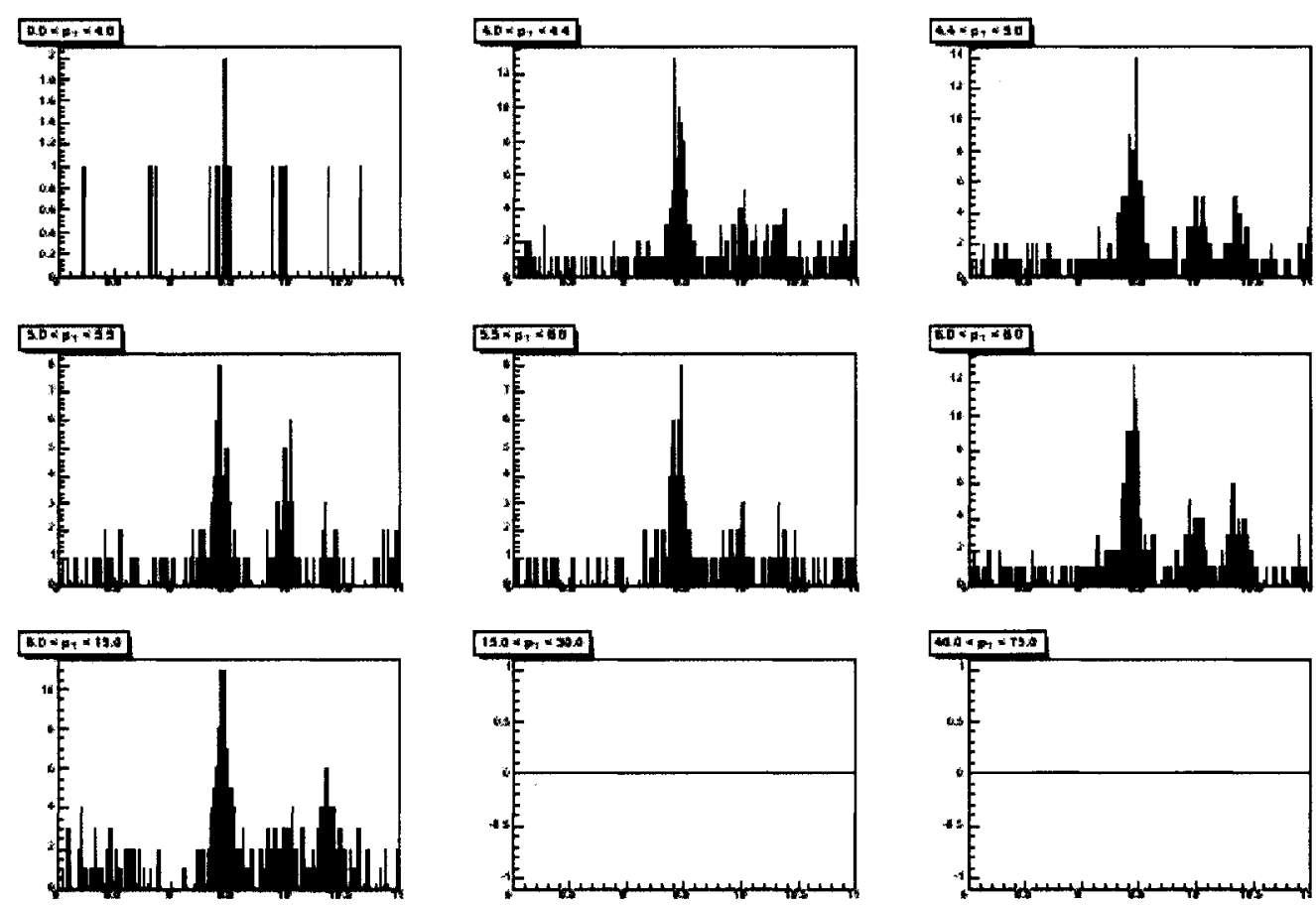

Figure C.2: $\Upsilon$ mass distributions in pt bins, used in efficiency calculations. Numerators are in blue, filled, histograms, and denominators in red, not filled, histograms. 
Appendix C. Resonances Used for gen6 Data ID Efficiencies
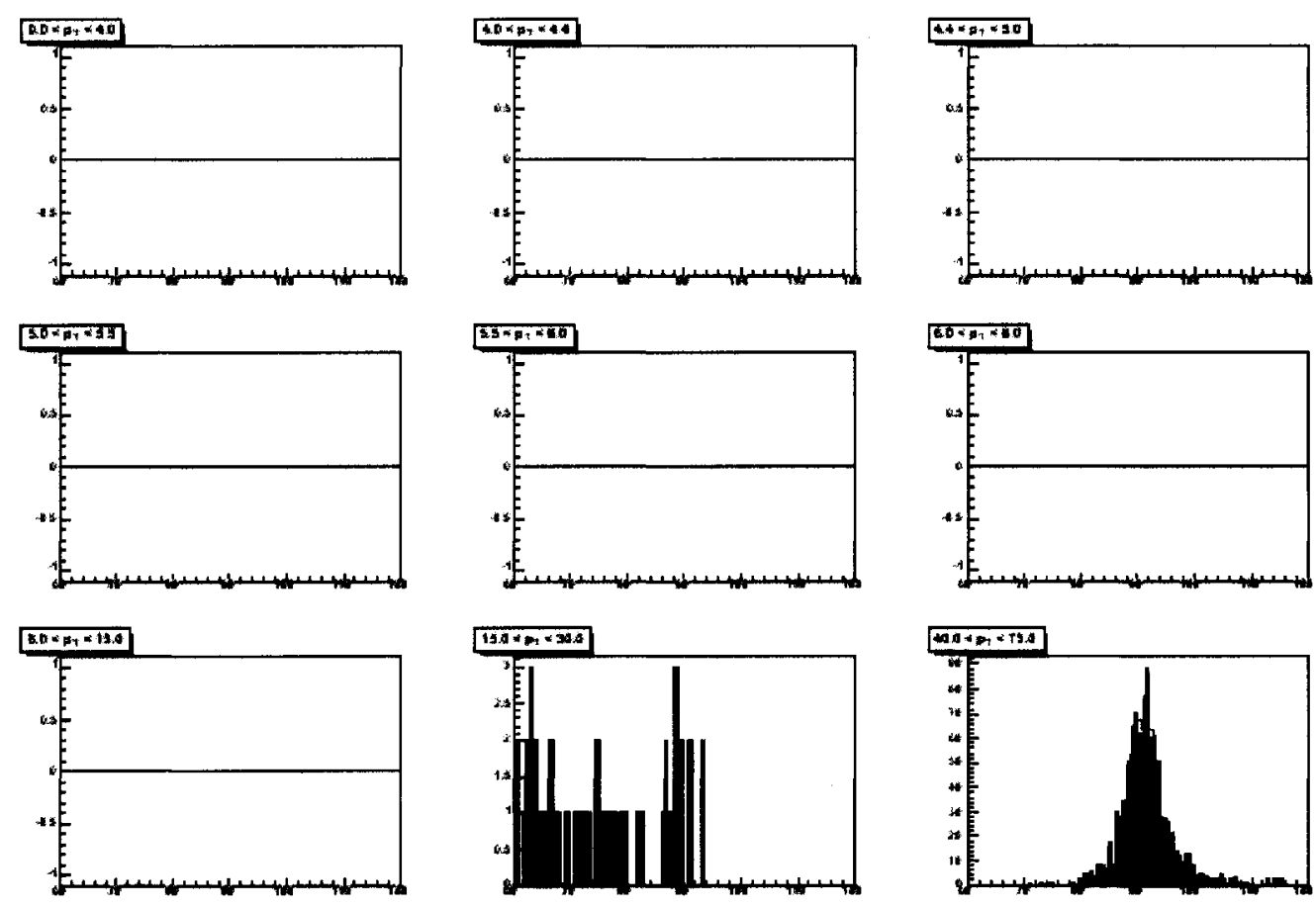

Figure C.3: $\mathrm{Z}$ mass distributions in pt bins, used in efficiency calculations. Numerators are in blue, filled, histograms, and denominators in red, not filled, histograms. 
Appendix C. Resonances Used for gen6 Data ID Efficiencies
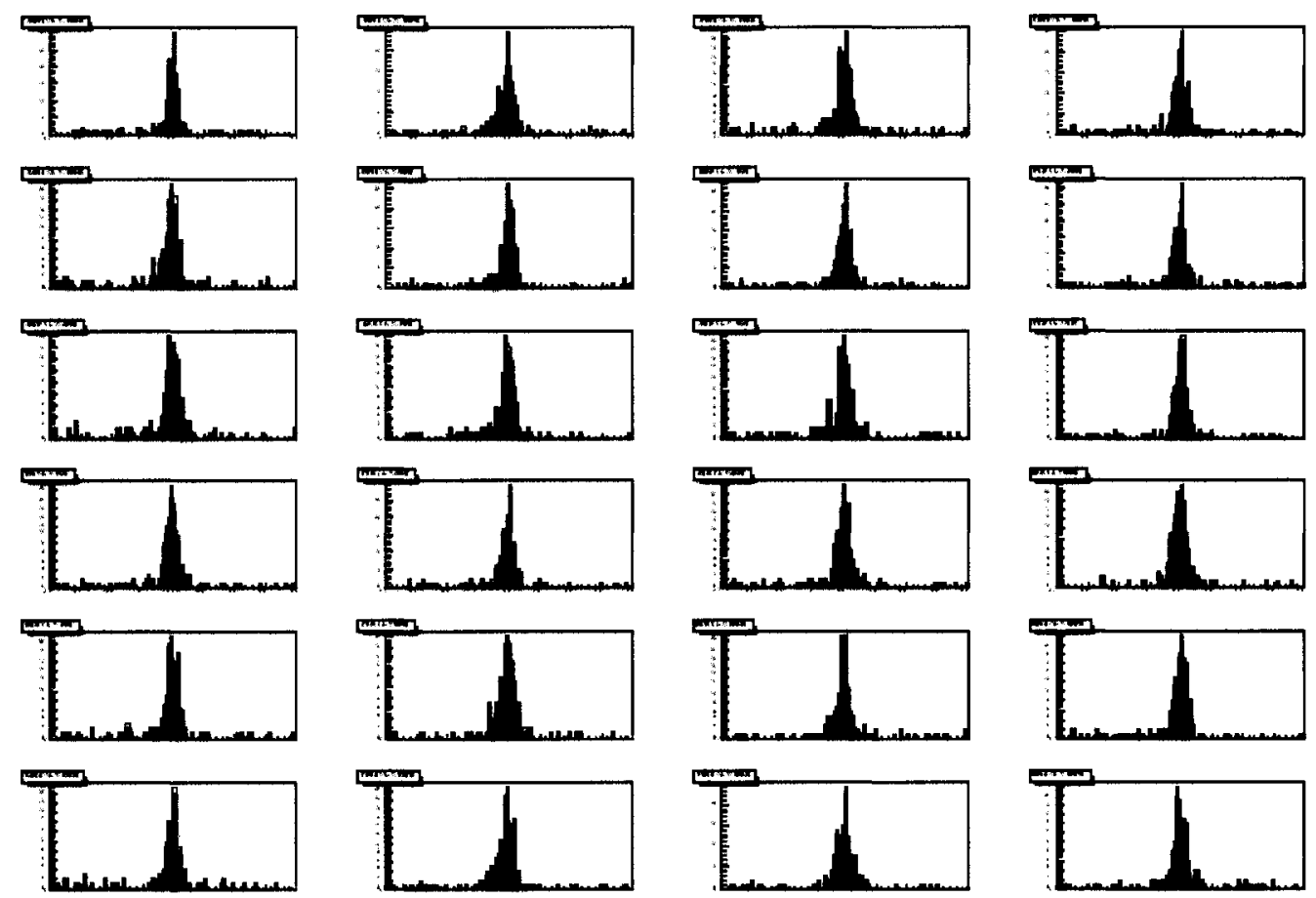

Figure C.4: $J / \psi$ mass distributions in phi bins, used in efficiency calculations. Numerators are in blue, filled, histograms, and denominators in red, not filled, histograms. 
Appendix C. Resonances Used for gen6 Data ID Efficiencies
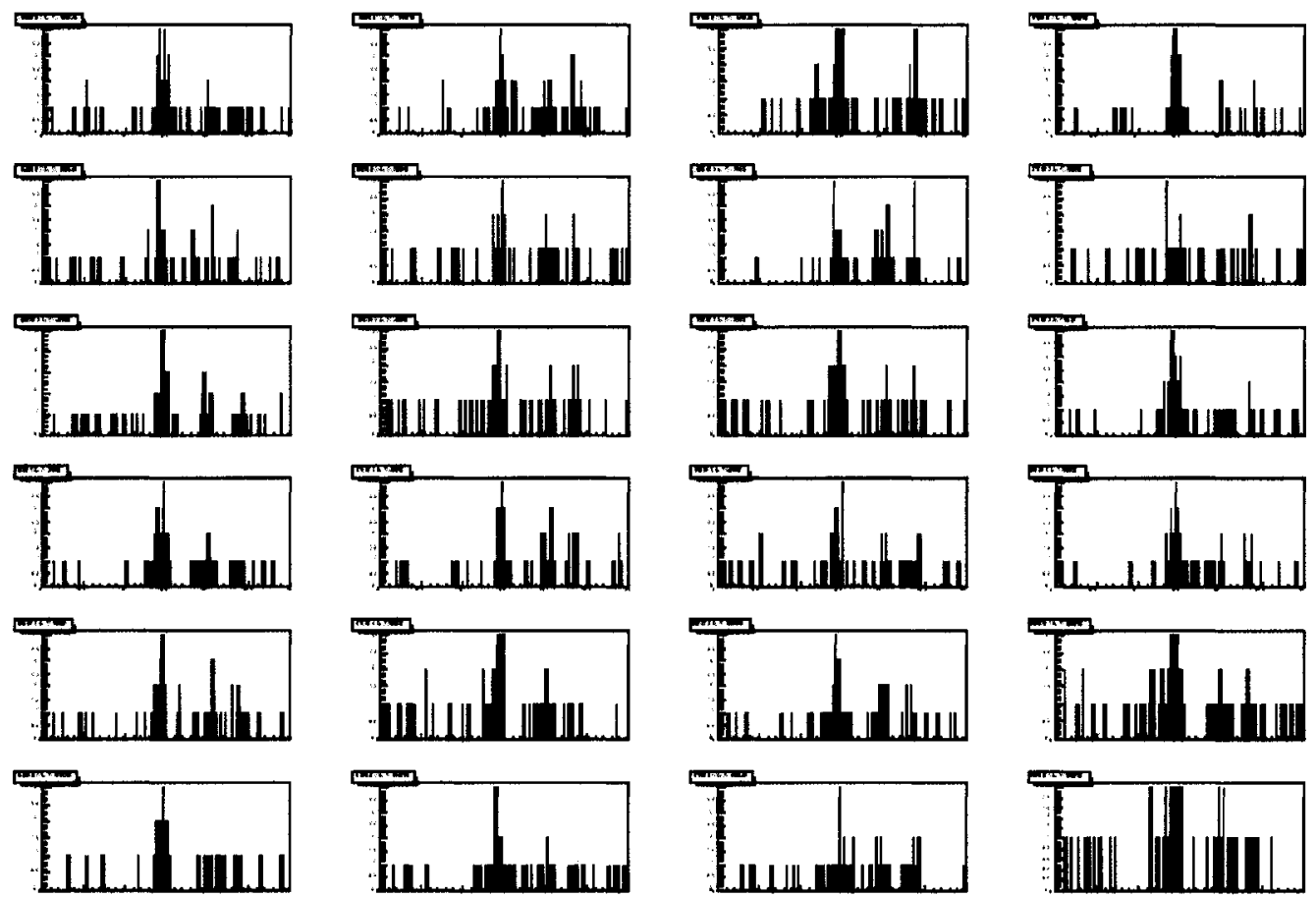

Figure C.5: $\Upsilon$ mass distributions in phi bins, used in efficiency calculations. Numerators are in blue, filled, histograms, and denominators in red, not filled, histograms. 

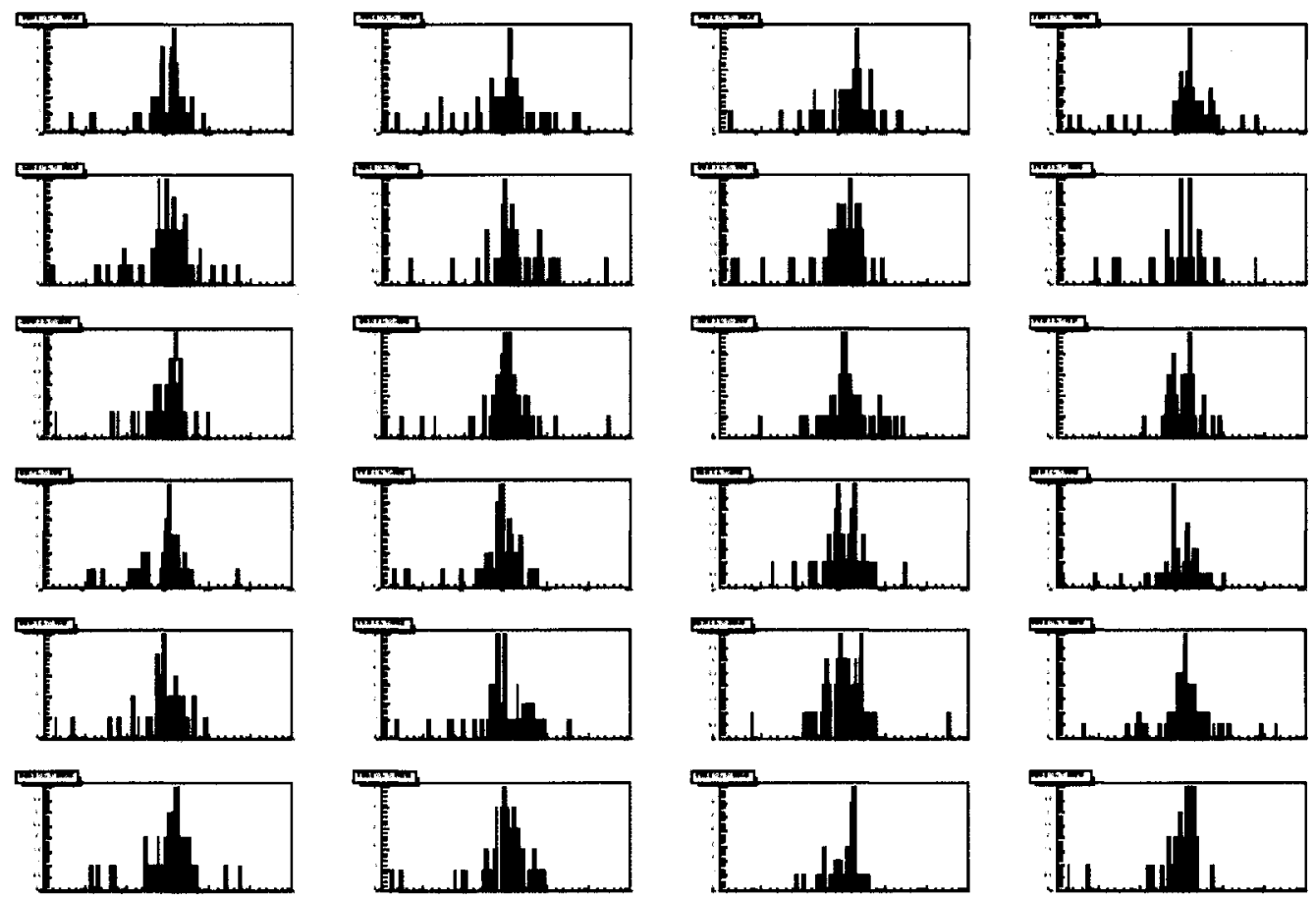

Figure C.6: Z mass distributions in phi bins, used in efficiency calculations. Numerators are in blue, filled, histograms, and denominators in red, not filled, histograms. 
Appendix C. Resonances Used for gen6 Data ID Efficiencies
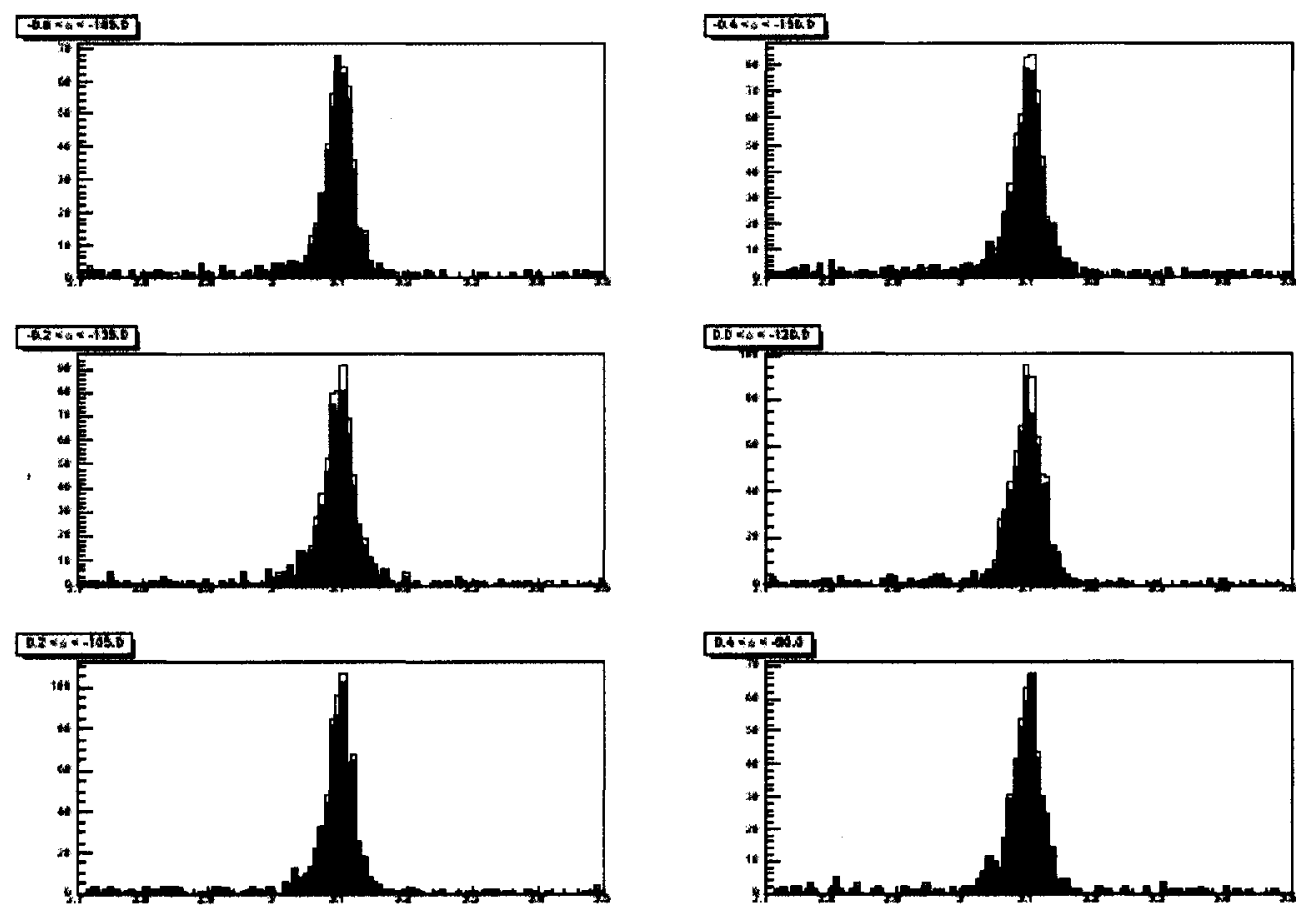

Figure C.7: $J / \psi$ mass distributions in eta bins, used in efficiency calculations. Numerators are in blue, filled, histograms, and denominators in red, not filled, histograms. 

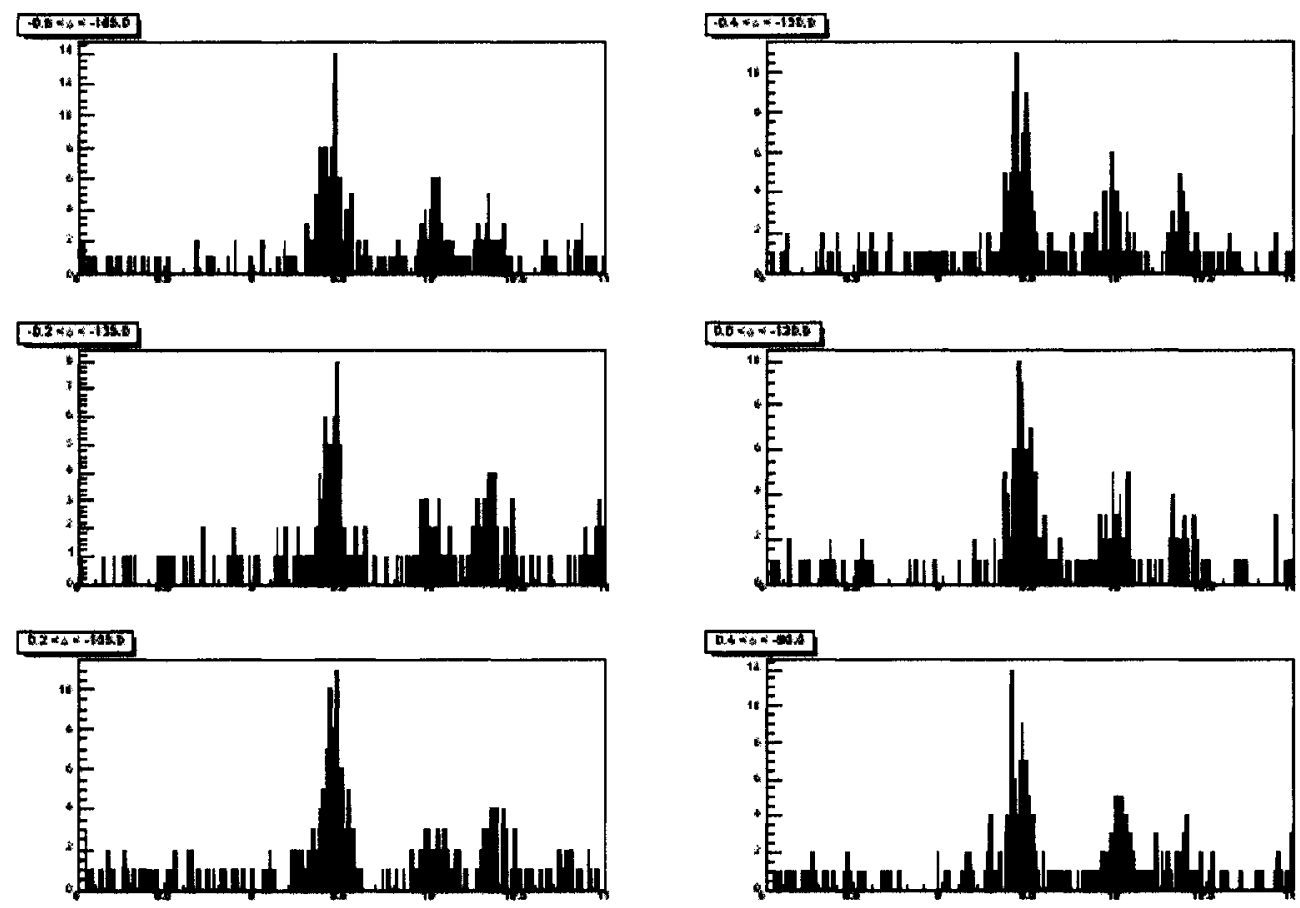

Figure C.8: $\Upsilon$ mass distributions in eta bins, used in efficiency calculations. Numerators are in blue, filled, histograms, and denominators in red, not filled, histograms. 
Appendix C. Resonances Used for gen6 Data ID Efficiencies
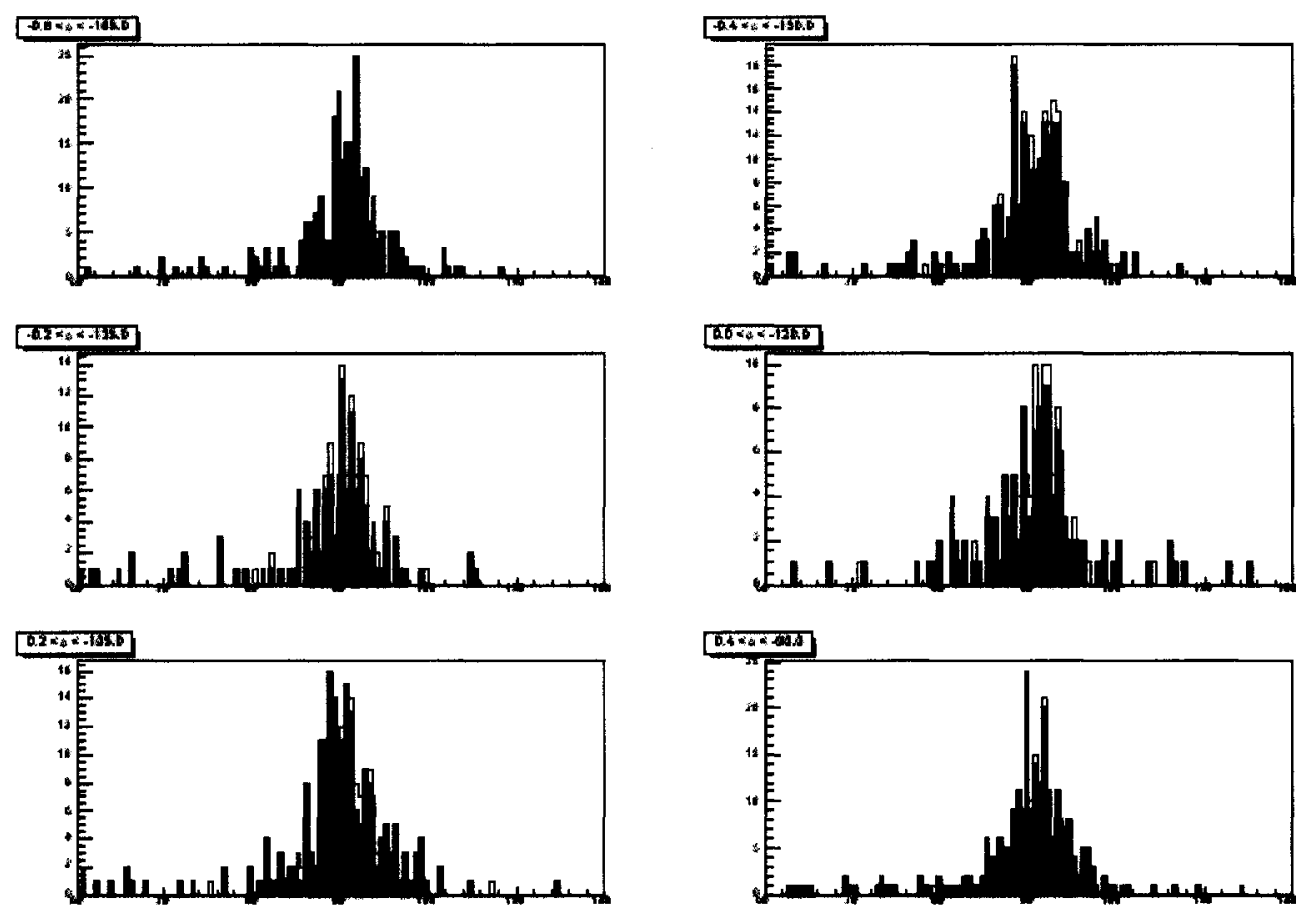

Figure C.9: Z mass distributions in eta bins, used in efficiency calculations. Numerators are in blue, filled, histograms, and denominators in red, not filled, histograms. 


\section{Appendix D}

\section{Cross Check of Muon ID}

\section{Efficiencies with High $p_{T}$ Group's}

\section{Results}

We cross check our method by measuring efficiencies of high $p_{T}$ electroweak cuts on the data in our sample, dilepton dataset edil0h. Unlike the cuts listed in tables 7.1, 7.2 and 7.3 , these cuts do not include CAL cut. We compared these measurements to the measurements performed on inclusive high $p_{T}$ muon dataset, bhmu0h, documented in [37]. The comparisons are shown in tables D.1 and D.2, and the plots of efficiencies vs $p_{T}$ in figure D.1. 
Appendix D. Cross Check of Muon ID Efficiencies with High $p_{T}$ Group's Results

\begin{tabular}{|lc|c|}
\hline cut \\
TOPGroup & $\epsilon$ & $\epsilon_{C D F 7956}$ \\
\hline EM & $0.979 \pm 0.005$ & $0.977 \pm 0.002$ \\
HAD & $0.989 \pm 0.004$ & $0.983 \pm 0.002$ \\
TRK & $1.000 \pm 0.003$ & $1.000 \pm 0$ \\
D0 & $0.992 \pm 0.003$ & $0.997 \pm 0.007$ \\
ISO & $0.968 \pm 0.006$ & $0.974 \pm 0.002$ \\
CMUDX & $0.953 \pm 0.006$ & $0.957 \pm 0.003$ \\
CMPDX & $0.974 \pm 0.005$ & $0.980 \pm 0.002$ \\
\hline ALL & $0.889 \pm 0.010$ & $0.898 \pm 0.004$ \\
\hline ALLISO & $0.858 \pm 0.011$ & $0.875 \pm 0.004$ \\
\hline
\end{tabular}

Table D.1: CMUP ID efficiencies of cuts used by the Top (high $\left.p_{T}\right)$ group and efficiencies of these cuts measured by the Top group and documented in CDF7956 (both for gen6 data).

\begin{tabular}{|lc|c|}
\hline cut $_{\text {TOPGroup }}$ & $\epsilon$ & $\epsilon_{C D F 7956}$ \\
\hline EM & $0.973 \pm 0.006$ & $0.967 \pm 0.003$ \\
HAD & $0.982 \pm 0.005$ & $0.980 \pm 0.002$ \\
TRK & $1.000 \pm 0.003$ & $1.000 \pm 0$ \\
D0 & $0.991 \pm 0.003$ & $0.998 \pm 0.001$ \\
ISO & $0.984 \pm 0.005$ & $0.971 \pm 0.003$ \\
CMXDX & $0.997 \pm 0.003$ & $0.995 \pm 0.001$ \\
\hline ALL & $0.942 \pm 0.008$ & $0.943 \pm 0.004$ \\
\hline ALLISO & $0.928 \pm 0.009$ & $0.916 \pm 0.004$ \\
\hline
\end{tabular}

Table D.2: CMX ID efficiencies of cuts used by the Top ( high $p_{T}$ ) group and efficiencies of these cuts measured by the Top group and documented in CDF7956 (both for gen6 data). 
Appendix D. Cross Check of Muon ID Efficiencies with High $p_{T}$ Group's Results
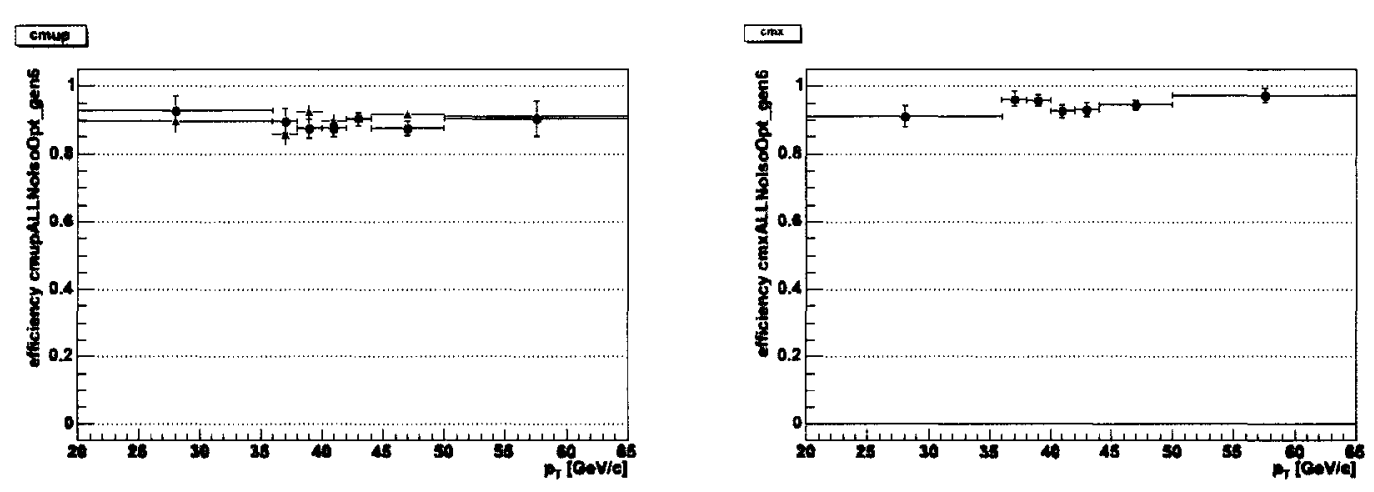

Figure D.1: CMU, CMUP, and CMX ID efficiencies of Top Group's all cuts as a function of $p_{T}$ of other leg (blue circles) and trigger leg (reg triangles), in the high $p_{T}$ region $\left(p_{T}>20 \mathrm{GeV} / c\right)$. 


\section{Appendix E}

\section{Efficiencies of Muon ID Cuts for \\ Low and High $p_{T}$ Regions for gen6}

In this Appendix, we show our measurment of efficiency of each cut in low and high $p_{T}$ region separately. 
Appendix E. Efficiencies of Muon ID Cuts for Low and High $p_{T}$ Regions for gen6
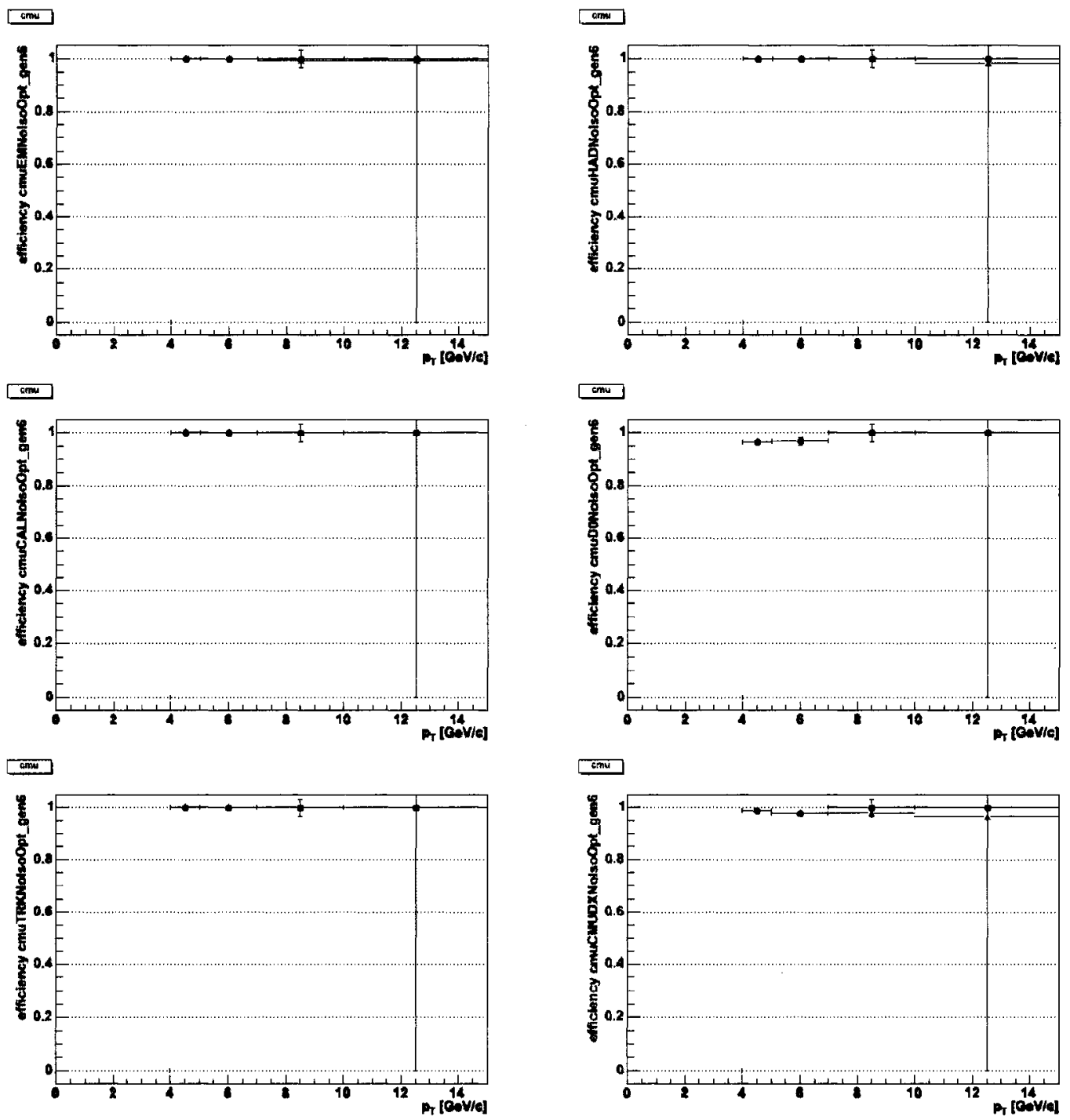

Table E.1: individual cut eff CMU low pt (EM,HAD,CAL,D0,TRK,CMUDX) for trigger (red) and other (blue) leg. 
Appendix E. Efficiencies of Muon ID Cuts for Low and High $p_{T}$ Regions for gen6
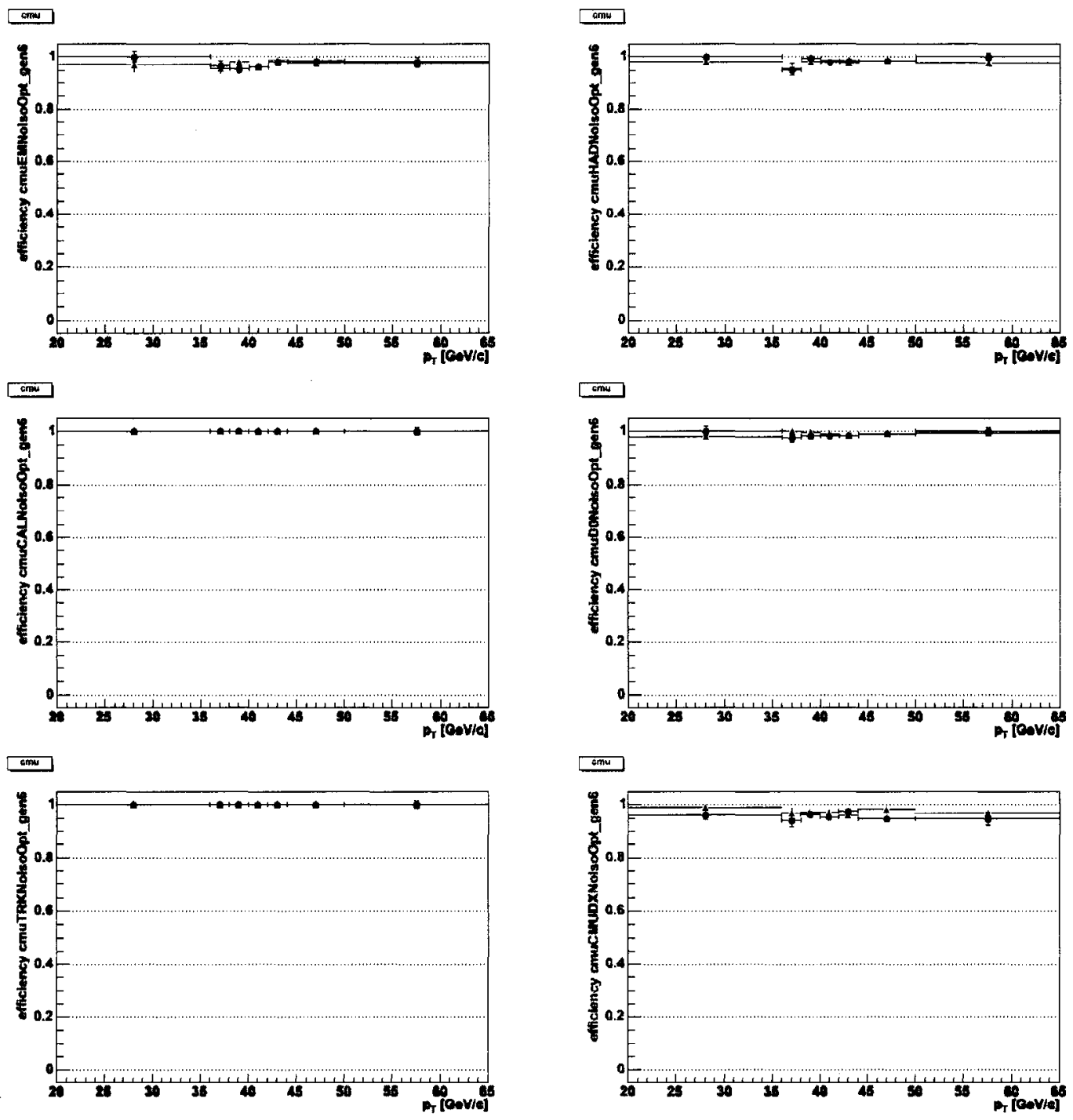

Table E.2: individual cut eff CMU high pt (EM,HAD,CAL,D0,TRK,CMUDX) for trigger (red) and other (blue) leg. 
Appendix E. Efficiencies of Muon ID Cuts for Low and High $p_{T}$ Regions for gen6
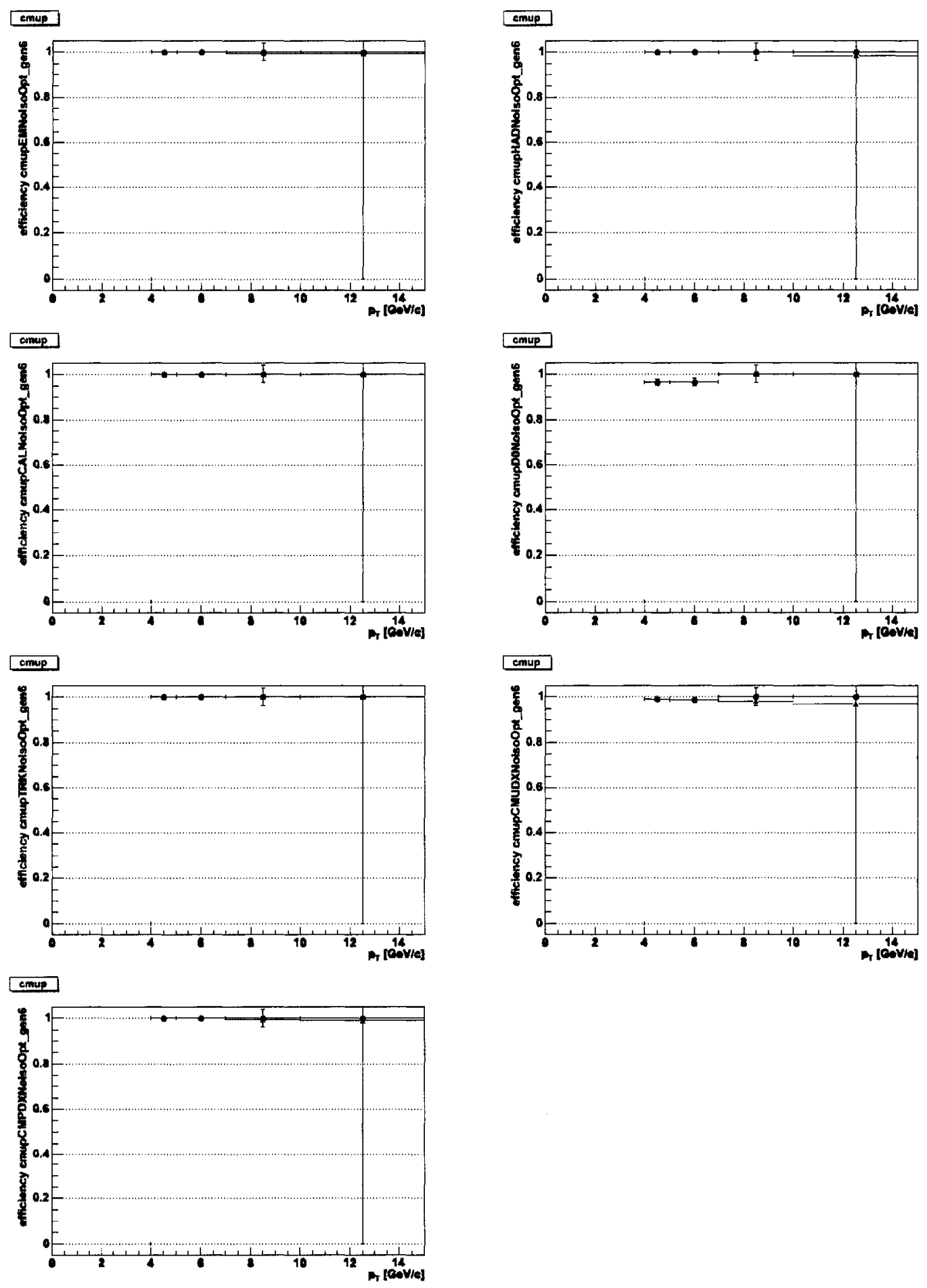

Table E.3: individual cut eff CMUP low pt (EM, HAD, CAL, D0, TRK, CMUDX, CMPDX) for trigger (red) and other (blue) leg. 
Appendix E. Efficiencies of Muon ID Cuts for Low and High $p_{T}$ Regions for gen6
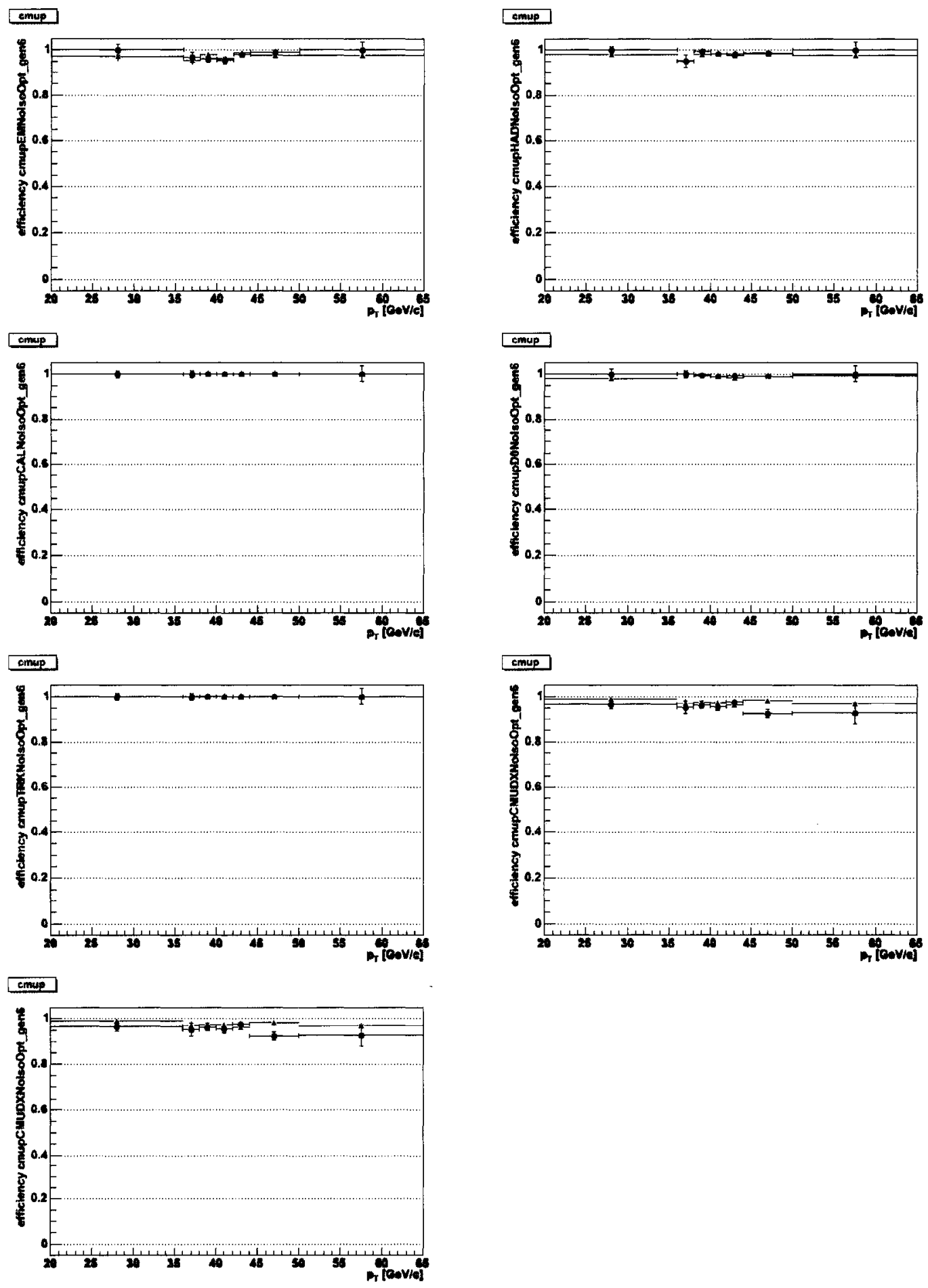

Table E.4: individual cut eff CMUP high pt (EM, HAD, CAL, D0, TRK, CMUDX, CMPDX) for trigger (red) and other (blue) leg. 
Appendix E. Efficiencies of Muon ID Cuts for Low and High $p_{T}$ Regions for gen6
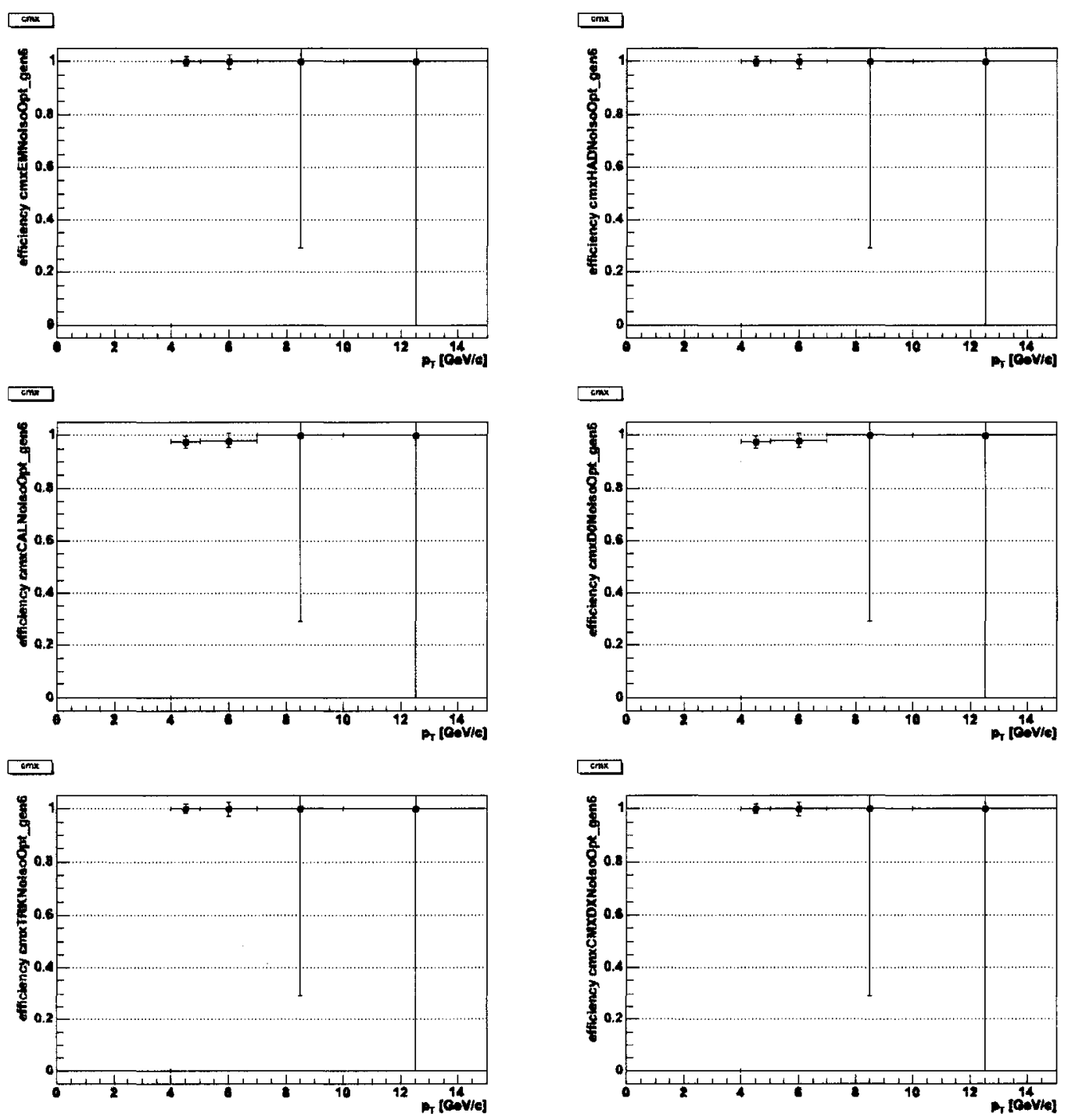

Table E.5: individual cut eff CMX low pt (EM,HAD,CAL,D0,TRK,CMXDX) for trigger (red) and other (blue) leg. 
Appendix E. Efficiencies of Muon ID Cuts for Low and High $p_{T}$ Regions for gen6
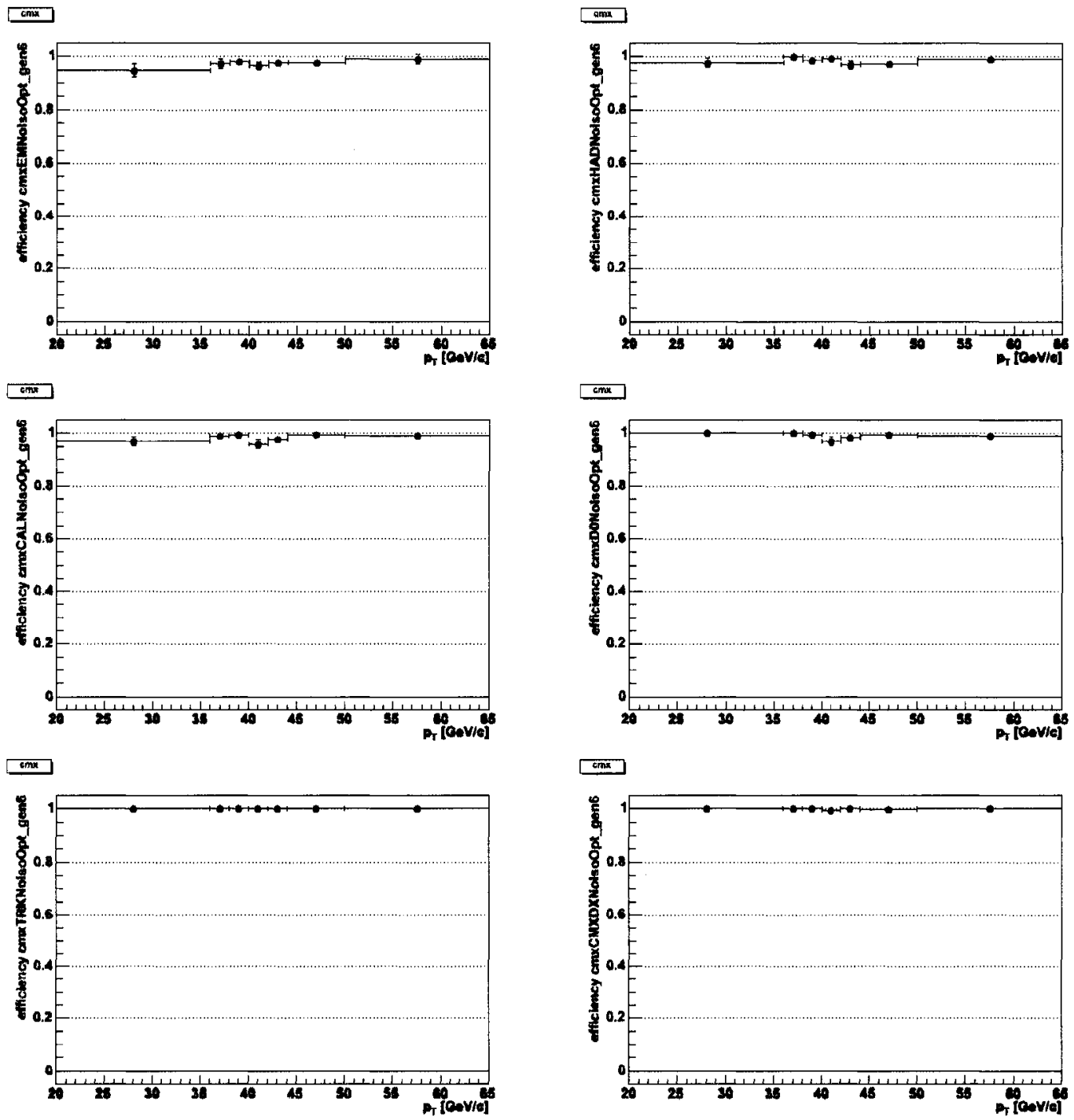

Table E.6: individual cut eff CMX high pt (EM,HAD,CAL,D0,TRK,CMXDX) for trigger (red) and other (blue) leg. 


\section{Appendix F}

\section{Comparisson of Efficiencies of}

\section{Muon ID Cut with High $p_{T}$ Group's}

In this Appendix, we show our measurment of efficiency of each cut used by Top Group in high $p_{T}$ region. 
Appendix F. Comparisson of Efficiencies of Muon ID Cut with High $p_{T}$ Group's
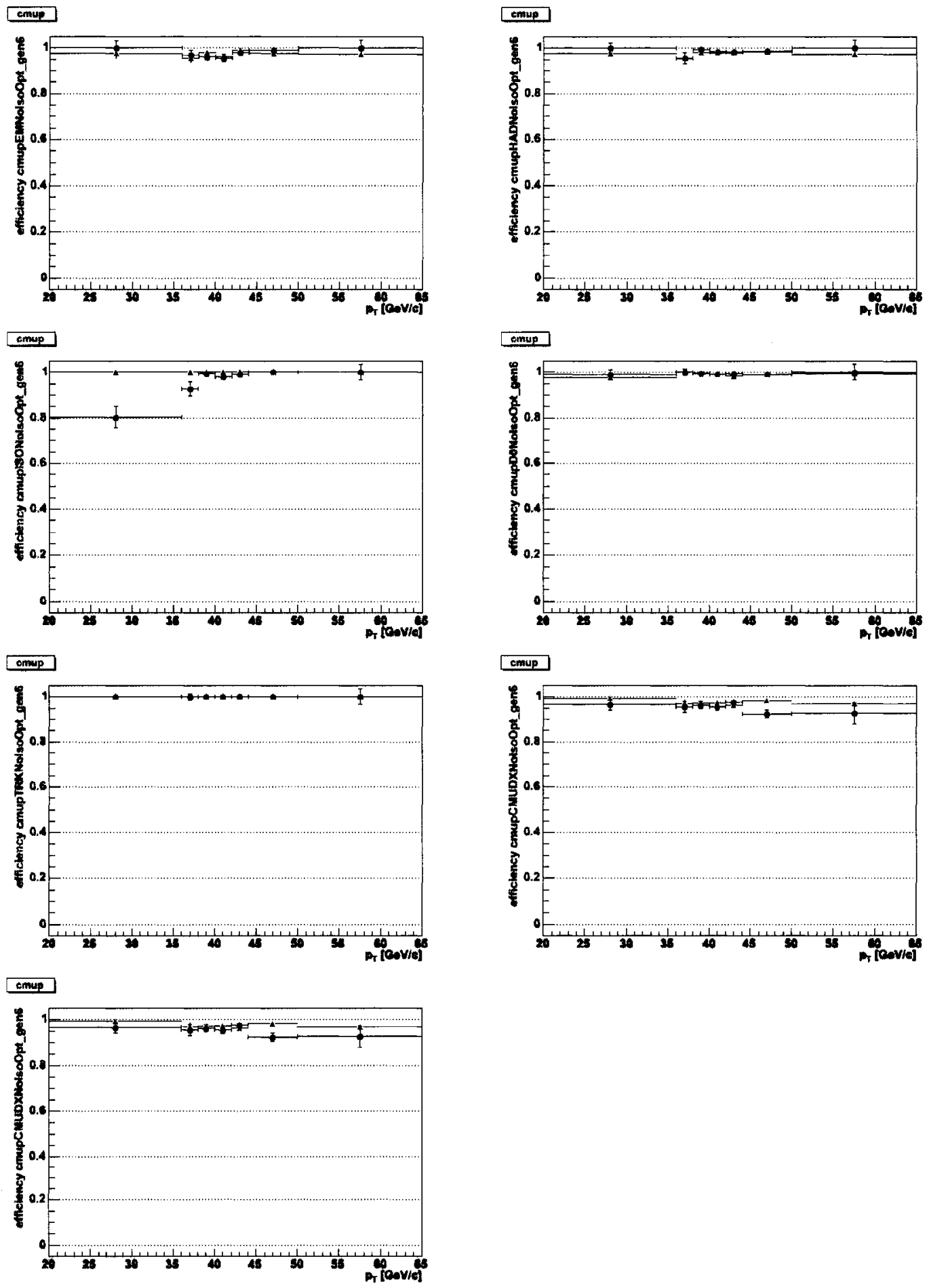

Table F.1: Top Group's individual cut eff CMUP high pt (EM, HAD, ISO, D0, TRK, CMUDX, CMPDX) for trigger (red) and other (blue) leg. 
Appendix F. Comparisson of Efficiencies of Muon ID Cut with High $p_{T}$ Group's
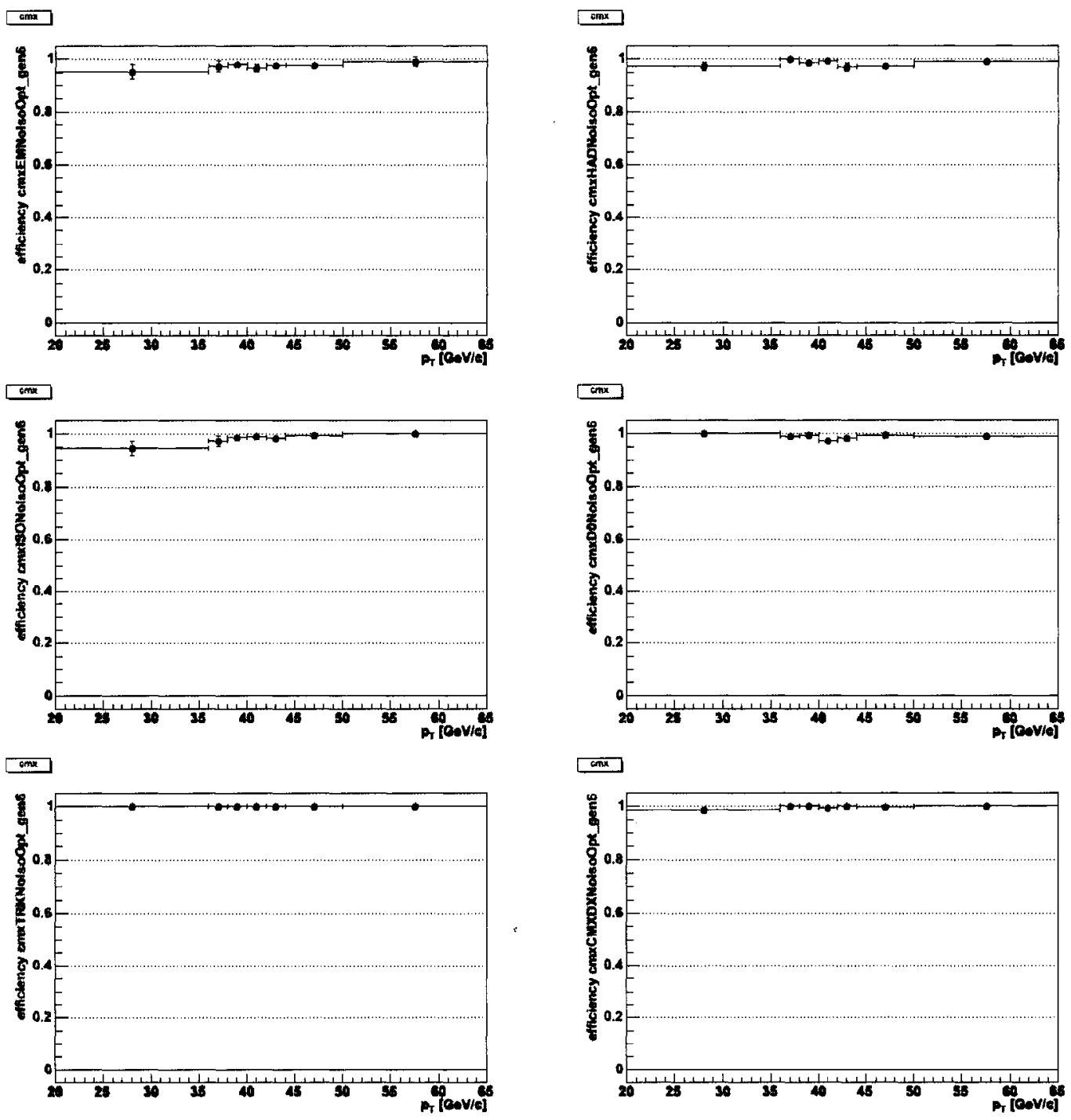

Table F.2: TopGroup's individual cut eff CMX high pt (EM, HAD, ISO, D0, TRK, CMXDX) for trigger (red) and other (blue) leg. 